UNIVERSITY OF CALIFORNIA

SANTA CRUZ

\title{
THE PHONOLOGY OF MORPHEME REALIZATION
}

\author{
A dissertation submitted in partial satisfaction \\ of the requirements for the degree of \\ DOCTOR OF PHILOSOPHY \\ in \\ LINGUISTICS \\ by
}

\section{Kazutaka Kurisu}

June 2001

The Dissertation of Kazutaka Kurisu is approved:

Professor Armin Mester, Chair

Professor Junko Itô

Professor Jaye Padgett

$\overline{\text { Vice Provost \& Dean of Graduate Studies }}$ 
Copyright @ by

Kazutaka Kurisu

2001 


\section{Table of Contents}

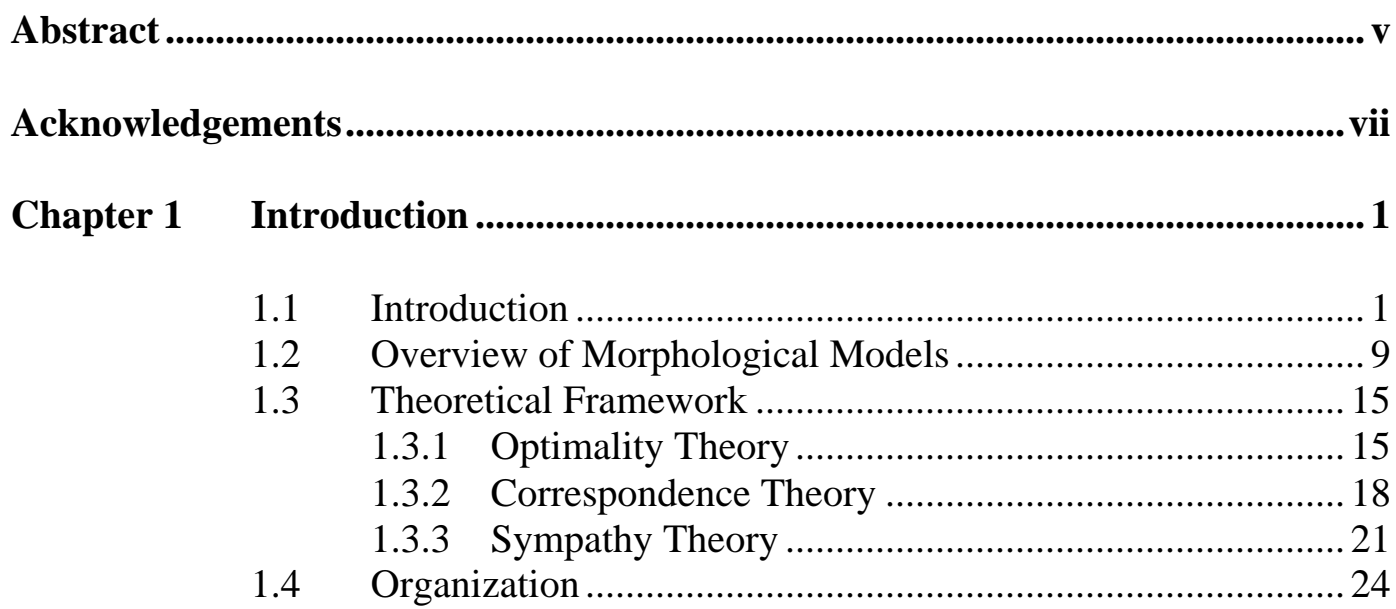

Chapter 2 Deriving Nonconcatenative Morphology …........................................... 27

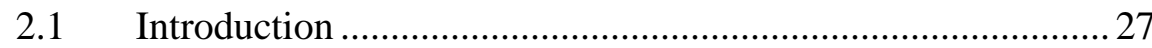

2.2 Anti-Faithfulness Effects.................................................. 29

2.3 Characterizing "Realize Morpheme"...................................... 37

2.4 Emergence of Nonconcatenative Morphology .......................56

2.5 Against Anti-Faithfulness Theory …....................................67

2.6 Summary ….............................................................. 76

Chapter 3 Truncatory Morphology _......................................................................79

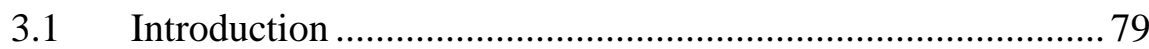

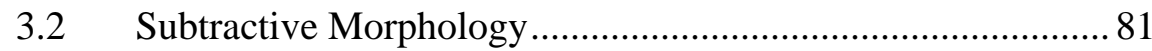

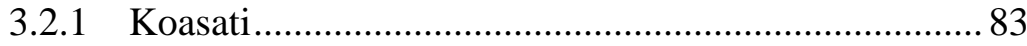

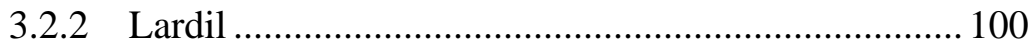

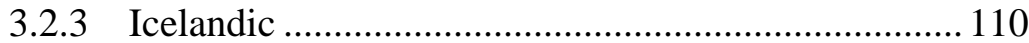

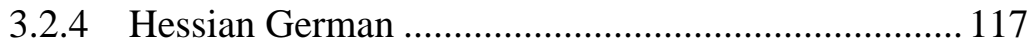

3.2.5 Summary …...................................................... 123

3.3 Templatic Truncation .................................................. 124

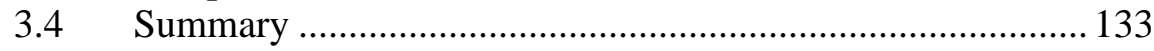

Chapter 4 Optimality of Nonconcatenative Allomorphs .................................. 135

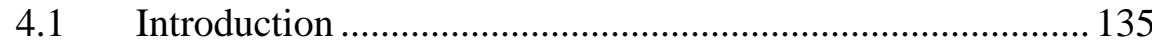

4.2 Nonconcatenative Allomorphs as Word Optimization ......... 137

4.3 The Continuative in Upriver Halkomelem ............................. 142

4.3.1 Facts and Descriptive Generalization....................... 143

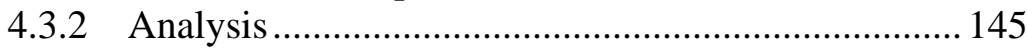




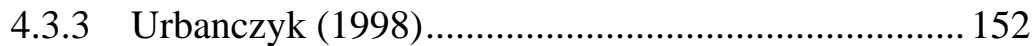

4.4 The Actual Aspect in Saanich ........................................... 156

4.4.1 Facts and Descriptive Generalization....................... 157

4.4.2 Analysis .......................................................... 159

4.4.3 Montler (1989) and Stonham (1994)....................... 164

4.5 The Incomplete Phase in Rotuman...................................... 167

4.5.1 Facts and Descriptive Generalization....................... 169

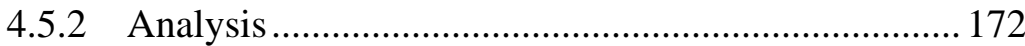

4.5.3 McCarthy (2000c) ................................................. 180

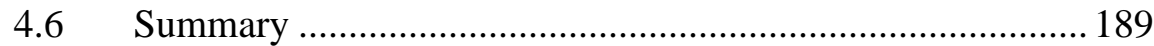

Chapter 5 Double Morphemic Exponence as Morphological Opacity......... 191

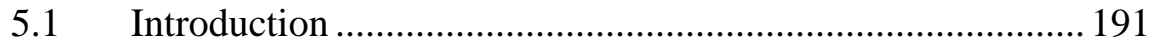

5.2 Morphological Opacity and Sympathy Theory ..................... 193

5.3 Dominant Affix Effects in Japanese.....................................2207

5.3.1 Facts and Descriptive Generalization.......................207

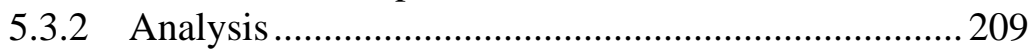

$5.4 \quad$ The Impersonal Formation in Chaha................................... 214

5.4.1 Facts and Descriptive Generalization.......................2 214

5.4.2 Morphemic Representation of the Impersonal .......... 216

5.4.3 Selector Constraint Revisited ................................. 222

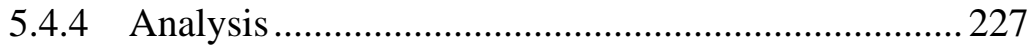

5.5 Affixation-Reduplication Interactions in Tagalog ............... 231

5.5.1 Multiple Functions of Affixation and Reduplication 231

5.5.2 Emergence of the Unmarked in Reduplication ......... 236

5.6 Phonological Polarity .......................................................2 245

5.7 Theoretical Predictions....................................................249

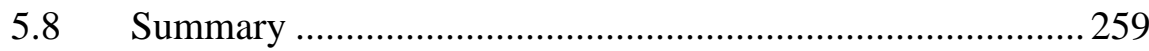

Chapter 6 Conclusion ...............................................................................................261

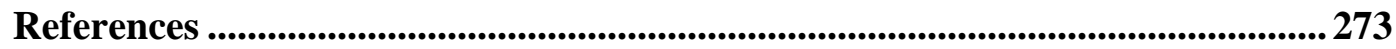




\section{Abstract \\ The Phonology of Morpheme Realization \\ Kazutaka Kurisu}

This dissertation addresses the question of how various types of morpheme realization are to be coherently explained within the framework of Optimality Theory (Prince and Smolensky 1993). Paying close attention to a-templatic nonconcatenative morphological processes such as subtractive morphology and umlaut, I develop a formal theory dubbed "Realizational Morphology Theory (RMT)".

The most important claim of this dissertation is that a REALIZE MORPHEME (RM) constraint plays a central role in a comprehensive and principled understanding of realizational morphology. In nonconcatenative morphology, its interactions with faithfulness constraints are of crucial importance. A second important proposal is the relativization of faithfulness constraints with respect to morphosyntactic categories. Given these two proposals, I demonstrate that the entire range of a-templatic nonconcatenative morphology is obtained through the ranking RM » Faith.

RMT not only covers morpheme realization in general but also has a number of desirable theoretical consequences. First, various descriptive devices such as zero morphs and floating features can be dispensed with, therefore simplifying the theory of morphology. Second, RMT is restrictive, eliminating unlikely morphological constructions. Especially, RMT eliminates the possibility of two simultaneous stem modifications. This argument is couched in terms of harmonic bounding (Prince and Smolensky 1993:176-178). This restrictiveness plays an important role in a critical evaluation of anti-faithfulness theory (Alderete 1999) since the latter predicts the existence of cases where a single morpheme receives more than one stem change. 
The organization of this dissertation is as follows. Chapter 1 is a general introduction, mapping out the overall goals in the context of previous works. Chapter 2 is devoted to the development of RMT. The remaining chapters are devoted to exemplification of RMT and to its further theoretical development. In chapter 3, I discuss morphological truncation. Chapter 4 discusses complementary distribution of nonconcatenative allomorphs, and in chapter 5, I examine constraint interactions resulting in two simultaneous phonological realizations of a single morpheme. Finally, chapter 6 summarizes the main results of this dissertation. 


\section{Acknowledgments}

A number of people have contributed to my completion of this dissertation at various stages. First of all, I express my deep gratitude to my dissertation committee: Armin Mester, Junko Itô and Jaye Padgett.

Armin Mester was one of the best advisors for me that I could imagine. He showed his strong interest in this dissertation from the beginning to the end. His challenging criticisms and insightful comments always inspired me. He gave me a number of invaluable suggestions to make the analysis shaper and solid. I believe that this dissertation would never have been here without his constant advising and encouragement.

Junko Itô has been a heroine of phonology for me. The first phonology paper that I read was her work on Japanese prosodic minimality. I remember that I was deeply impressed and started to get into phonology. It was really lucky for me that I had her as one of my committee members. Her comments were always large scaled and reminded me of the position of my dissertation within a large context.

Jaye Padgett has a very sharp eye to look at phonology. His comments and polite criticisms always got to the heart of my dissertation. His constant attitude to seek deeper explanations behind phonology has had tremendous influence on me throughout my graduate years at the University of California, Santa Cruz.

Besides my dissertation committee members, a number of people deserve special thanks. During my years at the University of California, Santa Cruz, other faculty members helped me in numerous dimensions: class instruction, paper writing, collaboration through an instructor-TA/RA relation and so forth. Various interactions with the faculty members were precious experiences for me to establish myself as a professional linguist. I express my deep gratitude to the department to foster such an 
active environment. I would like to thank in particular Judith Aissen, Donka Farkas and Jim McCloskey.

Among my fellows, Adam Ussishkin deserves special thanks. We were both writing a dissertation during the summer in 2000 . We regularly met and exchanged comments. He generously spent much time on reading my drafts and giving me invaluable comments. Nathan Sanders shared some research interest with me. Occasional informal discussion with him was very useful in developing various ideas. Rodrigo Gutierrez and Dylan Herrick have been both good listeners and friends.

Reflecting on the past five years, the former and present graduate students as well as visiting scholars have played an important role to me both in and outside linguistics. Listing just a few, they include Chris Gunlogson, Ryuji Harada, Takeru Honma, Daniel Karvonen, Motoko Katayama, Chris Kennedy, Brian Lindsey, Suzanne Lyon, Jason Merchant, Line Mikkelsen, Davina Ramirez, Philip Spaelti, Anne Sturgeon, Kunitoshi Takahashi, Emily Urban, Rachel Walker and Andy Wedel. Jennifer Smith, a visiting assistant professor, was also a good listener.

Some non-linguists also made a contribution to the successful completion of this dissertation. Among others, I would like to express my special thanks to Connie Creel, Ashley Hardisty and Tanya Honig for great administrative assistance as well as Paul Sosbee for his technical assistance for computers.

I benefited enormously from discussion with people outside the department as well. Among others, I thank John Alderete, Outi Bat-El and Paul Smolensky for listening to my ideas when they visited the University of California, Santa Cruz. I benefited indirectly from conversation with Stuart Davis, Paul Kiparsky, Larry Hyman and Peter Sells as well. Thanks to Haruka Fukazawa for her friendship. 
Various portions of this dissertation were delivered at the following linguistics conferences: Asian GLOW at Nanzan University (1999), Phonology Forum at Tokyo Metropolitan University (1999), the sixth meeting of the Phonological Society of Japan at Chiba University (2000), and WECOL at the California State University, Fresno (2000). I would like to thank those people who gave me invaluable feedback on these occasions.

Finally, but most of all, I need to express my deepest gratitude to my parents: Akinori and Takami Kurisu. They constantly gave me tremendous moral support and love. I hereby express my sincere gratitude to them for their generous understanding, support and love. This dissertation is dedicated to you. 


\section{Chapter 1}

\section{Introduction}

\subsection{Introduction}

This dissertation concerns issues surrounding the phonological realization of morphemes and develops a specific model dubbed "Realizational Morphology Theory" (RMT). Natural languages express the presence of a morpheme through some phonological manifestation, but the actually attested range of such phonological instantiations is wide. They can be roughly divided into two main classes: concatenative morphology and nonconcatenative morphology. Concatenative morphology depends upon affixation where the phonological material affiliated with distinct morphemes are sequentially agglutinated, as seen in the majority of cases involved in the plural formation in English (e.g., book $\approx b o o k s)$. Thus, concatenative morphology may roughly be equated with affixational morphology. On the other

hand, nonconcatenative morphology does not employ sequential concatenation of morphemes, such that the morpheme information contained in the surface form cannot be sequentially defined. The most famous and well-known example is binyan systems observed in Semitic languages like Arabic and Hebrew. [katab] 'write, perfective active', for instance, has been analyzed in generative morphology and phonology such that the entire form consists of the consonantal root $k t b$ and a verbal affix $a(a)$ (McCarthy 1979, 1981). Assuming that this view of the binyan is on the right track, the two morphemes involved in [katab] cannot be separated in a clear-cut manner since the phonological content of the two morphemes is intermingled within the word. 
Besides the binyan morphology exhibiting templatic effects, nonconcatenative morphology encompasses a wider range of a-templatic morphological phenomena. They include umlaut, subtractive morphology, morphological metathesis, suppletion, mutation, morphological haplology or fusion and so forth. Despite a great degree of different appearances on the surface, these phenomena share an essential property with the kind of nonconcatenative morphology observed in Semitic languages: phonological instantiations of the morphemes essentially cannot be demarcated in the output representation. For instance, umlaut as seen in the plural formation in German is phonologically parasitic upon a stem segment rather than being independent of stem segments (e.g., Mutter $\approx$ Mütter 'mother(s)'). Furthermore, part of the phonological material of the base undergoes deletion in subtractive morphology, as in the nominative formation in Lardil (e.g., [yalulu] $\approx$ yalul] 'flame'). Thus, no tangible phonological substance appears to phonologize the abstract concept denoted by the nominative morpheme. Phonological realization in nonconcatenative morphology is therefore substantially different from that involved in concatenative morphology.

Despite the superficial difference, what is shared by concatenative and nonconcatenative morphology is the fact that some phonological change appears to express the presence of a morpheme contained in the underlying representation. Given the fact that the presence of morphemes is denoted phonologically, various issues surrounding morpheme realization invoke morphology-phonology interactions.

In this dissertation, I examine how the relevant interactions should be formally captured, taking Optimality Theory (OT; Prince and Smolensky 1993) as the analytical framework. The primary goal is to achieve a comprehensive understanding of realizational morphology under the heading of RMT. Some important questions 
arise in this context. First of all, what does it mean that a morpheme has some phonological exponence on the surface? Adding new phonological material to the base (i.e., affixation) is of course one possibility, but it is not the only way attested in natural languages, as can be seen from a variety of nonconcatenative morphological processes such as those mentioned above. To capture the whole range of phonological exponence of morphemes attested in natural languages, concatenative and nonconcatenative morphology must receive a unified understanding. Given that some phonological substance associated with a given morpheme is attached to the base in concatenative morphology, morpheme realization in such cases is considered to be an issue of parsing the underlying material. But how are nonconcatenative morphological operations such as umlaut, subtractive morphology and so forth to be explained? In subtractive morphology, for example, some underlying element is elided rather than added, so no phonological substance can be recognized that is associated with the relevant morpheme. Thus, the central focus will be on the examination of nonconcatenative morphology, keeping a sharp eye on formal similarities and differences of concatenative and nonconcatenative morphology. In the subsequent study of nonconcatenative morphology, I especially focus on atemplatic nonconcatenative morphology which has attracted rather less attention in the earlier literature, but the ultimate goal is to propose a model to understand concatenative and nonconcatenative morphology in a unified fashion.

As will be argued in detail in chapter 2, my central claim is that a Realize Morpheme constraint (RM) proposed and employed in various contexts in the OT literature plays a pivotal role in RTM (for preceding works involving RM, see Samek-Lodovici 1993; Akinlabi 1996; Gnanadesikan 1997; Rose 1997; Walker 1998, 
2000; Piggott 2000; Kurisu 1999; 2000ab, 2001, to appear). Roughly speaking, RM requires every morpheme contained in the underlying representation to receive some phonological exponent on the surface. RM thus motivates overt phonological exponence of abstract morphemes. Given the fact that RM requires phonological parsing of underlying morphemes, it is best taken as a morphological faithfulness constraint.

Another important issue is how the exact range of morpheme realization is to be accounted for. Given both concatenative and nonconcatenative morphology (especially, a variety of phenomena subsumed under nonconcatenative morphology), it might appear at first glance that the range of actually attested phonological exponence of morphemes is not restricted. But it turns out that the range of options is quite restricted. It is an important aspect in constructing a model of realizational morphology that it does not suffer from an over-generation problem. In this light, it is important to review two influential morphological models: Item-and-Arrangement and Item-and-Process. The role of OT adopted in this dissertation becomes significant in this light because it offers an appropriate analytical device to restrict the possible range of morphological operations in a reasonable way. As I will discuss in section 1.2, Item-and-Arrangement and Item-and-Process models suffer from serious empirical and/or conceptual problems. I argue that RTM couched in the framework of OT successfully eschews such problems, motivating the necessity to frame RTM in OT.

Furthermore, what is the formal difference between purely phonological alternations and morphologically motivated phonological changes? Obviously, the difference is that the latter is sensitive to some morphological information while the 
former is not, descriptively speaking, but the question is how the grammar recognizes this distinction in a formal way. Under OT, as assumed throughout this dissertation, purely phonological alternations are motivated by markedness constraints ranked over competing faithfulness constraints. This has been already fairly established in the OT literature since Prince and Smolensky (1993). On the other hand, I will claim that morphologically conditioned phonological changes are governed by RM ranked over phonological faithfulness constraints. The idea is that the high ranked morphological faithfulness constraint forces violations of phonological faithfulness constraints. There has been a long-standing debate concerning whether morphology constitutes an independent module of the grammar separated from other modules such as syntax and phonology. This issue is not addressed in this dissertation, but it is true that a system must be developed which integrates morphology and phonology to achieve a satisfactory understanding of realizational morphology. RTM, embedded in OT, provides such a model since morphological and phonological constraints interact with one another within a single constraint hierarchy.

In addition to these fundamental questions, I address more advanced issues. First, a single morpheme often possesses various nonconcatenative allomorphs. For example, Upriver Halkomelem, a Salishan language, exhibits a complementary distribution of reduplication, prefixation, vowel lengthening, and stress shift to obtain phonological denotation of the continuative morpheme. This state of affairs is not surprising given the fact that affixational or concatenative morphology displays allomorphs. The only difference is that the relevant allomorphs depend on affixation or stem modifications. The parallelism is an area that has been understudied thus far. The central question is how the formal difference should be explained, still keeping 
the similarity that both concatenative and nonconcatenative allomorphs are surface phonological manifestations of a single morpheme which appear in complementary distribution. This is the topic of chapter 4.

Second, natural languages exhibit cases where a single morpheme receives two phonological exponents cooccurring in the same form. German plural formation is one such example. In the plural formation, the suffix -e or -er (phonetically, [ə]) is productively employed (e.g., $[\mathrm{kInt}]_{\text {Singular }} \approx[\mathrm{k} \text { ind-ə) }]_{\text {Plural }}$ 'child(ren)'), but umlaut often takes place on top of suffixation (e.g., $[\text { buux }]_{\text {Singular }} \approx[\text { büüç-əa }]_{\text {Plural }}$ 'book(s)'). I call such examples double morphemic exponence. The primary problem is how double morphemic exponence is to be accounted for. Given many cases where phonological realization of a morpheme is achieved solely by affixation (e.g., English plural formation), it is a mystery why an additional stem modification such as umlaut is called for. I address this question in chapter 5.

Addressing the issues raised above, I claim the following major points in this dissertation:

(i) Realizational morphology in general is successfully captured by OT. Given its basis to decide the optimal output form through constraint interactions, the intricate morphology-phonology interface in realizational morphology is explained by interactions of morphological and phonological constraints. (See section 1.2 for details.)

(ii) Nonconcatenative morphology exhibits anti-faithfulness effects, which crucially needs to be distinguished from faithfulness violations incurred in purely phonological alternations. Anti-faithfulness effects cannot be handled by phonological faithfulness and markedness constraints alone. RM plays a 
key role as the driving force of various nonconcatenative stem modifications such as umlaut and morphological subtraction. They are motivated by RM ranked over some phonological faithfulness constraint (i.e., RM » Faith), the specific surface realization of a morpheme depending on the specific phonological faithfulness constraint. (See chapter 2 for details.)

(iii) Natural languages exhibit two types of morphological truncation: subtractive morphology and templatic truncation. They are remarkably different because what is deleted is phonologically invariable in subtractive morphology whereas the residue remaining after deletion is constant in templatic truncation. Nevertheless, they are governed by the same fundamental ranking: RM » Max. The difference is gained by the absence/presence of a set of prosodic markedness constraints that jointly require outputs to take a certain prosodic shape. If such constraints outrank Max, templatic truncation obtains while they do not dominate Max in subtractive morphology. (See chapter 3 for details.)

(iv) A single morpheme often shows allomorphs that are not affixational (e.g., metathesis and subtractive morphology are in complementary distribution). Such nonconcatenative allomorphs are best considered as languages' effort to minimize the markedness value of the overall word. Unlike affixational allomorphs, nonconcatenative allomorphs are more global since the overall word shape is taken into consideration. Nevertheless, they are governed by the same fundamental mechanism as affixational allomorphs in the sense that the least marked allomorph is chosen as the optimal one. (See chapter 4 for details.) 
(v) Morphemes are often phonologically manifested by two surface exponents (double morphemic exponence), as in the plural formation in German. In double morphemic exponence, some stem change is invoked in addition to affixation. Sympathy theory, as proposed by McCarthy (1999), plays a central role in capturing those cases. Double morphemic exponence takes place because the affix is made invisible or opaque for the computation of RM satisfaction/violation, and therefore, some independent morpheme realization is called for, an effect dubbed here "morphological opacity". This is formally implemented by establishing Stem $\equiv \mathrm{PrWd}$ as the selector constraint which demands the coextensiveness of a stem and the prosodic word. (See chapter 5 for details.)

(vi) Anti-faithfulness theory, as developed by (Alderete 1999), is seemingly attractive given the fact that nonconcatenative morphology exhibits antifaithfulness effects. But it is not a viable theoretical device to capture the range of phenomena discussed here since it suffers from a number of empirical and conceptual problems while RTM overcomes them. (See the overall dissertation, section 2.5 in particular.)

In addition to these major claims, a number of theoretical questions and consequences are discussed in various portions of this dissertation. To close this section, I map out the organization of the rest of this chapter. In section 1.2, I review two morphological models proposed by structural linguists: Item-and-Arrangement and Item-and-Process. They are both concerned with how word formation should be viewed. I discuss strengths and weaknesses of each model, claiming that neither model is satisfactory as a general theory of realizational morphology. In this context, 
I argue that OT provides an analytical tool suitable to understand it more plausibly. In section 1.3, I briefly review the framework of OT assumed throughout this dissertation. OT has undergone numerous development since the original proposal made by Prince and Smolensky (1993), but I restrict my review to three issues of relevance here: basic mechanisms to obtain outputs, correspondence theory (McCarthy and Prince 1995), and sympathy theory (McCarthy 1999). Finally, section 1.4 closes this chapter by discussing the overall organization and a brief synopsis of this dissertation, fleshing out what was discussed in this section more concretely.

\subsection{Overview of Morphological Models}

Affixation or additive morphology is undoubtedly the most productive, and therefore, the most widely attested morphological process across languages. This is because morphologically richer information is generally conveyed by the attachment of one or more affixes: the more complex the morphosyntactic information is, the more additional morphemes are employed. For example, in English, a singular noun is normally taken as the base of its plural form, and the latter is derived with an additional suffix $-s$. The principle that semantically more implies morphologically more is called constructional iconicity or diagrammaticity (Dressler 1987; Spencer 1991). Since Bloomfield (1933), it was a widely accepted view by structural linguists that word formation is agglutinating. This morphological model, called Item-andArrangement (henceforth, IA), has the following properties (Hockett 1954:214): (i) A linguistic form is either simple or composite, (ii) a simple form is monomorphemic, and (iii) a composite form consists of two or more immediate constituents, each of which occupies a certain position in the construction. Under the IA model, a 
morpheme is viewed as a morphologically indivisible primitive atom, and every kind of word formation consists exclusively of concatenation of morphemes. Given that prefixation and suffixation are highly productive crosslinguistically, the IA theory would be a successful approach to predominant word formation processes.

But it is by no means the case that additive or concatenative morphology is the only morphological operation observed in human languages. A variety of problems flow from the principle of morphemic structure in IA when a full range of word formation phenomena attested in natural languages is considered. Root-and-pattern morphology advanced by McCarthy $(1979,1981)$ is one such example (cf. Harris 1941 for earlier insights, and Chomsky 1951 for a transformational account for the phenomenon). Semitic languages like Arabic and Hebrew exhibit interdigitation of more than one morpheme within a word. In the verbal morphology paradigm in Arabic, for instance, [katab] 'write, perfective active' consists of a root $k t b$ and a verbal affix $a(a)$. They are arranged in such a manner that the root consonants and the affixal vowels are intermingled with one another rather than being concatenated linearly. Because the segments of the two morphemes are interspersed, their precedence relation cannot be sequentially defined. This kind of word formation (socalled nonconcatenative morphology) poses a serious challenge to the IA theory.

A similar line of argument against IA can be provided by infixation, circumfixation, and probably also discontinuous morphemes at a phrasal or sentential level discussed by Harris (1945), because morphemes are not continuous in these cases. Another problem comes from truncation, a morphological phenomenon widely discussed since the emergence of prosodic morphology (McCarthy and Prince 1986 et seq.). This is a theory aimed at explaining how morphological and phonological 
determinants of linguistic form interact with each other in a grammatical system. This theory made a significant contribution to morphology-phonology investigations in the sense that it offered an explicit theory of analyzing morphological operations in terms of prosodic units. Prosodic circumscription (McCarthy and Prince 1990), a further development of extrametricality and an important subcomponent of prosodic morphology, provided a powerful tool for analyzing morphological phenomena (e.g., infixation) from a prosodic perspective. Prosodic circumscription is of significant importance in the sense that it uncovered that morphological operations are often phonologically conditioned in a systematic way. Truncation is one such morphological phenomenon and invokes clipping of part of a base, so the idea of constructional iconicity or diagrammaticity is completely subverted here. These observations together seriously endanger the empirical plausibility of the IA model.

Along the same lines, other morphological operations which crucially depend on non-affixation provide further rich empirical evidence against the IA theory. Such phenomena include subtractive morphology, metathesis, vowel changes (i.e., ablaut, umlaut, and suppletion), haplology (fusion), and suprasegmental changes (i.e., stress and tone shifts). These nonconcatenative phenomena do not contain a clearly identifiable morph, because they involve deformation of a base rather than any addition of phonological substance. Especially, subtractive morphology does the opposite of morpheme concatenation: deletion of part of a base. Compared with affixation, the nonconcatenative morphological processes are far less productive across languages, but they constitute strong empirical data against the IA view of structuralism that all morphemes are indivisible atomic units composed of phonological strings whose linear arrangement creates words. 
The argument above indicates that morphological processes cannot be exclusively characterized in terms of concatenation, and has led a number of researchers to criticize IA (Nida 1948; Hockett 1947, 1950, 1954; Janda 1984; Anderson 1988, 1992). An alternative view regards word formation as consisting of a set of processes. This is a model known as Item-and-Process (henceforth, IP) theory. Its properties are encapsulated as follows (Hockett 1954:227): (i) A linguistic form is either simple or derived, (ii) a simple form is a root, and (iii) a derived form consists of one or more underlying forms to which a process has been applied. Under this approach, a certain morpheme does not have to contain any phonologically tangible substance, because word formation is considered as a set of transformations. In nonconcatenative morphology, the IP model applies a rule to a base to obtain the surface representation. In concatenative morphology, on the other hand, a rule attaches a morpheme to an already existing form. Thus, the IP theory can capture a wider scope of data than the IA theory.

This seems to strongly suggest the superiority of IP over IA. The IP model has its own potential problems, however. As pointed out by Anderson (1992:63), IP is potentially less restrictive than IA. He argues that we run the risk of weakening the whole theory if various morphological processes other than the limited class of rules of simple affixation are admitted. In other words, we expect that, without proper delimitation of the power of possible transformations, any kind of morphologically conditioned phonological operation is observed somewhere as long as they can be formulated as transformational rules. However, a language is unlikely to exist which, for example, metathesizes onset consonants and the rime regularly in all syllables to express a morphosyntactic category (e.g., $/ \mathrm{C}_{1} \mathrm{~V}_{1} \mathrm{C}_{2} \cdot \mathrm{C}_{3} \mathrm{~V}_{2} / \rightarrow\left[\mathrm{V}_{1} \mathrm{C}_{2} \cdot \mathrm{C}_{1} \mathrm{~V}_{2} \mathrm{C}_{3}\right]$ ), despite the 
fact that the relevant transformational rule can be easily written. Similar unlikely morphological processes can be easily multiplied. This indicates that the IP model suffers from conceptual and empirical problems while the IA model has empirical difficulties. Hockett (1954) concludes that the two theories are both unsatisfactory.

This overview would suffice to highlight the fact that the nature of word formation has not received unanimous understanding. At a more fundamental level, the question is what morphemes are. It is evident from the controversy aroused between IA and IP that a satisfactory answer cannot be found by looking at additive or concatenative morphology alone. Thus, a comprehensive examination of the overall range of morphological phenomena needs to be done, crucially considering how nonconcatenative morphological operations are implemented.

The emergence of the derivational version of generative morphology and phonology has aroused debate concerning the formal treatment of nonconcatenative morphological processes. In the linear model originated by SPE (Chomsky and Halle 1968), reduplication was handled by a set of transformational rules to copy a string to the left, to the right, or in the middle of the base (Aronoff 1976; Carrier 1979; Lieber 1981). But this is again subject to the criticism of unrestrictiveness. Subsequently and alternatively, reduplication was seen as affixation where what is affixed is a CV skeleton or prosodic template (Marantz 1982). By reducing reduplication to affixation, the fear of permitting any random transformation would be avoided. Furthermore, umlaut as observed in German, for instance, was dealt with in such a way that there is a front vowel suffix /-i/ or a floating feature [-back] in the underlying representation as the trigger of the umlaut process. Various other morphological processes might receive a similar treatment potentially, but such 
derivational accounts would not yet be able to handle subtractive morphology. Floating features are a very abstract and extremely powerful device as the trigger of phonological changes, but how would subtractive morphology be derived in a principled manner? Given deletion of part of the base, there would be no possible way to represent the relevant trigger in terms of an affix or floating feature. This shows that even fairly articulated derivational approaches are not satisfactory either.

In this dissertation, I claim that OT offers an appropriate analytical vessel. Martin (1988) observes that the range of phonological changes involved in morphological phenomena is exactly the same as that found in purely phonological phenomena. This is a natural state of affairs given the fact that the presence of morphemes is denoted phonologically. As briefly mentioned in the preceding section and fleshed out in more detail later, nonconcatenative morphological effects are obtained through RM » Faith. As will be reviewed in section 1.3.2, the range of universal faithfulness constraints has been quite established since the emergence of correspondence theory (McCarthy and Prince 1995). Adopting correspondence theory embedded in OT thus provides a way to understand the parallelism between morphologically motivated phonological changes and purely phonologically defined ones. RTM couched in the framework of OT overcomes empirical and conceptual problems encountered by IA and IP. The specific phonological manifestation of a morpheme is the outcome resulting from constraint interactions.

An important implication is that whether morphemes are sequentially arranged or not does not matter for the grammar. Under the RMT model developed here, RM plays a central role, and its definition will be formulated in such a way that strict linear order of morphemes does not play any role. This means that the crucial 
empirical problem of the IA model arising from the view that morphemes are sequentially divisible is avoided in RTM. Moreover, RTM eschews the serious overgeneration problem of the IP model. While the kind of transformational operations postulated in the IP model is not constrained because the possible range of processes is not restricted, the range of possible morphological operations can be intrinsically limited in RTM by assuming exactly the phonological faithfulness constraints that seem to have been established in the OT literature. In effect, RTM provides a model of realizational morphology which encompasses exactly the range of phonological realization of morphemes attested in natural languages.

\subsection{Theoretical Framework}

As stated, RTM is framed in Optimality Theory (OT; Prince and Smolensky 1993). In this section, I provide a brief overview of this framework. This section consists of three portions. In section 1.3.1, I provide a description of some essential theoretical architecture of OT. The other two parts are concerned with two sub-theories: correspondence theory advanced by McCarthy and Prince (1995) (section 1.3.2) and sympathy theory proposed by McCarthy (1999) (section 1.3.3). Correspondence theory, a theory on faithfulness constraints, plays a central role throughout this dissertation whereas sympathy theory is of relevance in chapter 5.

\subsubsection{Optimality Theory}

The elementary theoretical design of OT (Prince and Smolensky 1993) consists in constraint interactions and constraint violability. Going back originally to SPE (Chomsky and Halle 1968), earlier generative phonological theories view the output 
production as a result of applying a series of rules (and inviolable representational constraints such as the OCP and repair strategies) to a given input. Such derivational models maintain serialism from the input to the output, going through a number of intermediate representations, as schematized in (1).

Derivational Theory

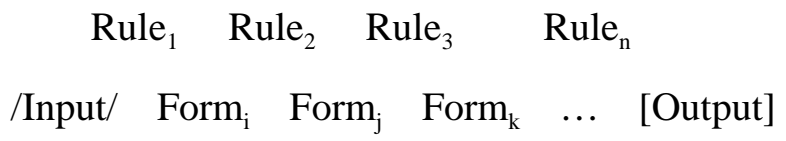

OT departs from derivational models in a number of respects. First, the OT grammar contains no rules or repair strategies. Rather, the essential ingredient producing outputs is universal constraints. Second, the output production is parallel in the OT grammar, meaning that there is no intermediate representation between the input and the output. Third, constraints are potentially all violable, and even the eventual output may violate them.

The OT grammar consists of three components: Gen, Con, and Eval (Prince and Smolensky 1993). Gen is a function that generates a potentially unlimited number of output candidates. The candidates yielded by Gen differ in the degree of the similarity to the given input, and they may take any form as long as they conform to the primitive structural restrictions that are never infringed upon universally, the principle known as inclusiveness (McCarthy and Prince 1993b:1). Con is a pool containing a set of constraints. Constraints are considered to be universal, so no language-specific constraint is admitted. All systematic differences among languages are explained through different constraint rankings. Constraints are ranked with respect to their importance, and each language has its own constraint hierarchy distinct from all others. Finally, Eval is responsible for choosing an ultimate output 
for a given input against the given constraint ranking. Eval evaluates comparative well-formedness of all candidates, and the most harmonic one is selected as the winner (i.e., the output). Since constraints are all violable, the best candidate does not have to satisfy all. Rather, it must be the most harmonic with respect to the ranked constraints. The OT grammar is schematically shown as in (2).

OT grammar

Gen $\left(\right.$ Input $\left._{\mathrm{i}}\right) \rightarrow\left\{\right.$ Cand $_{1}$, Cand $_{2}, \ldots$, Cand $\left._{\mathrm{n}}\right\}$

$\operatorname{Eval}\left(\left\{\operatorname{Cand}_{1}, \operatorname{Cand}_{2}, \ldots, \operatorname{Cand}_{\mathrm{n}}\right\}\right)=$ Output $_{\text {Real }}$

The function of Eval is visually illustrated in the form of tableau, as in (3).

\begin{tabular}{|ll|l|c|c|}
\hline & /Input/ & Constraint $_{1}$ & Constraint $_{2}$ & Constraint $_{3}$ \\
\hline \hline a. $\quad$ (r) & Candidate $_{1}$ & & & $*$ \\
\hline b. & Candidate $_{2}$ & & $* !$ & \\
\hline c. & Candidate $_{3}$ & $* !$ & & \\
\hline
\end{tabular}

Suppose that Candidate $_{1}$ through Candidate $_{3}$ are generated by Gen, and the job of Eval is to select the optimal form. In (3), constraints are ranked in the order of importance from left to right, meaning that Constraint ${ }_{1}$ and Constraint Core $_{3}$ the most

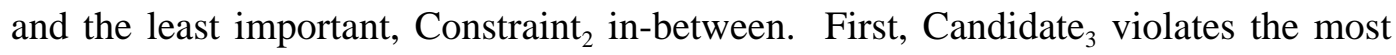
important constraint, and therefore, it is ruled out from the competition first of all. Between the remaining two candidates, Candidate ${ }_{1}$ is more harmonic since it violates only Constraint $_{3}$ which is ranked beneath Constraint ${ }_{2}$ violated by Candidate $_{2}$. Constraints are violable, and indeed, the best form violates a constraint here. But the ranking is strict in the sense that any number of violations of lower ranking constraints does not make up for a violation of a higher ranking constraint. The set of 
constraints is universal, but their ranking is not. Thus, the failed candidates in (3) can be chosen as the optimal form in other languages depending upon a permutation of the ranking. In this simple example, any of the three candidates appears as the winning form depending on which constraint is ranked the lowest. If Constraint ${ }_{2}$ is

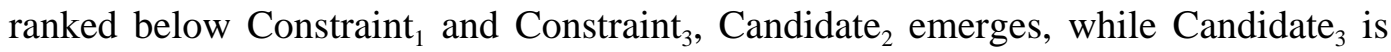
opted for if Constraint ${ }_{1}$ is dominated by Constraint d $_{2}$ and Constraint C. The winner $_{3}$ expected by Eval is indicated by a pointing finger. Stars indicate constraint violations, and exclamation marks show that the relevant constraint violations are fatal. Finally, shading shows that the satisfaction/violation in the cell is irrelevant to the selection of the eventual output form.

\subsubsection{Correspondence Theory}

Throughout this dissertation, correspondence theory, as proposed by McCarthy and Prince (1995), plays an essential role. OT constraints are largely grouped into two classes: markedness constraints and faithfulness constraints, and correspondence theory concerns the latter. Markedness constraints solely penalize phonologically marked structures or representations, but faithfulness constraints demand two corresponding structures or representations to be identical. Any conceivable elements (i.e., segmental, subsegmental, and suprasegmental) stand in correspondence, and identity of those elements is computed in various dimensions, including input-output (Faith-IO), output-output (Faith-OO), base-reduplicant (Faith-BR), and basetruncatum (Faith-BT). Following McCarthy and Prince (1995:262), correspondence of strings is defined as in (4), where $S_{1}$ and $S_{2}$ refer to an element belonging to two dimensions. The idea of correspondence is applied to subsegmental (featural) and 
suprasegmental (prosodic) elements as well, indicating that $S_{1}$ and $S_{2}$ in (4) may be any phonological element.

(4) Given two strings $S_{1}$ and $S_{2}$, correspondence is a relation $\Re$ from the elements of $S_{1}$ to those of $S_{2}$. Elements $\alpha \in S_{1}$ and $\beta \in S_{2}$ are referred to as correspondents of one another when $\alpha \Re \beta$.

Specific faithfulness constraints in correspondence theory are listed in (5) (McCarthy and Prince 1995:370-372). They prohibit divergence of correspondents in various dimensions.
a. $\quad$ Max
Every element of $S_{1}$ has a correspondent in $S_{2}$.
b. Dep
Every element of $S_{2}$ has a correspondent in $S_{1}$.
$\begin{array}{ll}\text { c. Ident- }[\mathrm{F}] & \text { Correspondent segments have identical values for the feature } \mathrm{F} \text {. }\end{array}$
d. Contiguity
The portion of $\mathrm{S}_{2}$ standing in correspondence forms a contiguous string.
e. $\quad\{$ Left/Right $\}$-Anchor $\left(\mathrm{S}_{1}, \mathrm{~S}_{2}\right)$
Any element at the designated periphery of $S_{1}$ has a correspondent at the designated periphery of $\mathrm{S}_{2}$.
f. Linearity
$\mathrm{S}_{1}$ is consistent with the precedence structure of $S_{2}$, and vice versa.
g. Uniformity
No element of $S_{2}$ has multiple correspondents in $S_{1}$.
h. Integrity
No element of $S_{1}$ has multiple correspondents in $S_{2}$.

Max and Dep are constraints which militate against deletion and epenthesis respectively. Thus, given /pit/ as the input, for instance, [pi] violates Max because the final segment /t/ is deleted in the output whereas [pitu] violates Dep by virtue of the fact that the final segment $[u]$ has no correspondent in the input. 
Ident-[F] demands complete identity of featural values of correspondents. [F] is a variable replaced by various concrete features such as [voice] and [coronal]. For example, [pid] violates Ident-IO-[voice] given that /pit/ is the input and that the final output segment $[\mathrm{d}]$ is the correspondent of /t/ in the input.

Contiguity requires that the linear order of input elements be preserved in the output representation. McCarthy and Prince (1995) distinguish two types of Contiguity constraints (i.e., I-Contiguity against skipping and O-Contiguity militating against intrusion), but this distinction is not important in the subsequent discussion. Contiguity defined in (5d) corresponds to O-Contiguity in McCarthy and Prince's (1995) terms, and this is what is relevant in this dissertation. It militates against any intrusion and is violated by forms such as [piat] for the input /pit/ since /i/ and /t/ contiguous in the input are not contiguous in the output because of the presence of an intervening segment [a].

Anchor requires that the element occupying either edge appear at the same edge in the output. Given prefixal monosyllabic reduplication and the input/pit/, [pipit] satisfies Anchor-L in the base-reduplicant dimension because the left edge segment in the reduplicant (i.e., [p]) occupies the left edge of the base as well. But [ipit] violates Anchor-L since the left edge segments of the reduplicant and the base are not the correspondents of each other.

Linearity is a constraint which bans metathesis since it dictates that precedence relations in the input must be preserved in the output. Thus, for the input /pit/, forms such as [ipt] and [pti] infringe on Linearity since the sequential relations holding among the three input segments are not completely preserved. 
Finally, Uniformity and Integrity are mirror-imaged constraints. Uniformity bans a configuration where a single output element has more than one correspondent in the input (i.e., no coalescence) whereas Integrity prohibits more than one output element from corresponding to one and the same input element (i.e., no breaking). For example, for $/ \mathrm{pi}_{\mathrm{i}} \mathrm{a}_{\mathrm{j}} \mathrm{t} /,\left[\mathrm{pe}_{\mathrm{i}, \mathrm{j}} \mathrm{t}\right]$ violates Uniformity since two input vowels share a single segment as their correspondent. By contrast, Integrity is violated by $\left[\mathrm{pi}_{\mathrm{i}} \mathrm{a}_{\mathrm{i}} \mathrm{t}\right.$ for the input / $\mathrm{pe}_{\mathrm{i}} \mathrm{t} /$ because two output segments have one and the same input segment as their input correspondent.

\subsubsection{Sympathy Theory}

Finally, I review sympathy theory which will play a central role in chapter 5. It is a sub-theory of OT advocated by McCarthy (1999) to handle phonological opacity (Kiparsky 1971, 1973). In this section, I review the fundamental mechanism of the theory and introduce technical terms employed in chapter 5. Among others, McCarthy discusses Tiberian Hebrew in which the interaction of $e$-epenthesis and :deletion exhibits phonological opacity (Malone 1993). The former operates to epenthesize a vowel $e$ between word-final consonants whereas a glottal stop undergoes deletion in the syllable coda. In derivational terms, this is illustrated in (6).

a. $\quad e$-epenthesis

$$
\varnothing \rightarrow \text { V/C_C\# (e.g., /melk/ } \rightarrow \text { [melek] 'king') }
$$

b. i-deletion

$$
? \rightarrow \varnothing /]_{\sigma} \quad \text { (e.g., /qara?/ } \rightarrow \text { [qara] 'he called') }
$$

These rules potentially interact with each other in a counter-bleeding manner, as shown in (7). The rule relation is counter-bleeding since -deletion, if applied first, destroys the environment of $e$-epenthesis. Under parallel OT, this is problematic 
because the surface representation does not have any justification to have the epenthesized vowel by virtue of the fact that it does not contain a consonant cluster word-finally. In McCarthy's terms, this state of affairs is called "non-surfaceapparency".

$$
\begin{array}{ll}
\text { Underlying representation: } & / \mathrm{de} J / \\
\begin{array}{l}
e \text {-epenthesis: } \\
\text { i-deletion: }
\end{array} & \begin{array}{l}
\text { de } \int \mathrm{e} \text { ? } \\
\text { de } \int \mathrm{e}
\end{array} \\
\text { Surface representation: } & \text { [de } \left.\int \mathrm{e}\right]
\end{array}
$$

The problem here becomes clearer given the following consideration. The fact that $e$-epenthesis occurs to break up a consonant cluster motivates *Complex » Dep-V. Furthermore, a syllable-final glottal stop is prohibited, and therefore, CodaCond (i,e., *? $]_{\sigma}$ ) is assumed to be ranked over Max-C. As demonstrated in (8), this ranking provides a surface transparent candidate (i.e., [def]) as the winner, contrary to fact. This form is better than [de $\left.\int e\right]$ (the desired winner marked by $\left.:\right)$ ) since the latter incurs an excrescent constraint violation in Dep-V. Comparing (8b)

\begin{tabular}{|c|c|c|c|c|c|}
\hline & $/ \operatorname{dej} ? /$ & CodaCond & *Complex & Max-C & Dep-V \\
\hline a. & $\operatorname{de} \int ?$ & $* !$ & $*$ & & \\
\hline b. & $\operatorname{de} \int$ & & & * & \\
\hline c. & $\operatorname{de} \int \mathrm{e}$ ? & *! & & & $*$ \\
\hline d. $\theta$ & $\operatorname{de} \int e$ & & & $*$ & $* !$ \\
\hline
\end{tabular}
and (8d), the latter is harmonically bounded by the former in the sense of Prince and Smolensky (1993:176-178), and no ranking permutation ameliorates the situation.

The innovative proposal made by McCarthy (1999) is that OT has a system to evaluate correspondence between candidates (inter-candidate correspondence) in 
addition to other various correspondence relations such as input-output, basereduplicant and so forth. Descriptively, the fact that epenthesis takes place in the eventual output is motivated by the fact that it imitates the epenthetic property of deje:, an intermediate representation in the derivational sense. On the other hand, de/ does not share this property with deje;, and this is exactly the fatal fault of the transparent candidate. This intuitive idea is formally implemented through the notion of sympathy.

The initial step is to grant special status to de/e: (called sympathy candidate) although it loses the competition because of its violation of CodaCond. The sympathy candidate is chosen by a designated constraint called selector constraint. The sympathy candidate is selected among the candidates which satisfy the selector constraint. Among those forms, the most harmonic one with respect to other constraints is selected as the sympathy candidate. In Tiberian Hebrew, Max-C serves as the selector constraint, which is marked by ${ }^{3}$. Considering (8), only (8a) and (8c) satisfy Max-C, and the latter is more harmonic with respect to other constraints since (8a) violates $*$ Complex which is satisfied by $(8 c)$. Since all other candidates violate the selector constraint, the violation of high ranked CodaCond does not matter for the purpose of choosing the sympathy candidate.

The next step is to determine the constraint which forces correspondence of all candidates with the designated sympathy candidate, again marked by Because $(8 b)$ and (8d) are discriminated by whether an epenthetic vowel is present or not, the relevant constraint is Max-8 $\mathrm{O}-\mathrm{V}$. This constraint requires all candidates to possess correspondents of all vowels of the sympathy candidate. This constraint is satisfied by (8d) but is violated by (8b). As demonstrated in (9), this sympathy proposal 
succeeds in selecting the desired candidate as the optimal form, eschewing the problem caused by harmonic bounding. In effect, the actual output is made optimal by virtue of the fact that it is sympathetic for a particular failed candidate through an inter-candidate correspondence constraint.

\begin{tabular}{|c|c|c|c|c|c|}
\hline$/ \operatorname{de} \int ? /$ & CodaCond & *Complex & Max-8 $8-\mathrm{O}$ & Max-C & Dep-V \\
\hline $\operatorname{de} \int ?$ & $* !$ & $*$ & $*$ & & \\
\hline $\operatorname{de} \int$ & & & $* !$ & $*$ & \\
\hline c. de $\int \mathrm{e}$ ? & $* !$ & & & & * \\
\hline d. de $\int \mathrm{e}$ & & & & $*$ & $*$ \\
\hline
\end{tabular}

\subsection{Organization}

The rest of this dissertation consists of five chapters. Chapter 2 outlines Realizational Morphology Theory (RMT). RMT accounts for the mechanism behind phonological realization of morphemes in general. Putting special focus on a-templatic nonconcatenative morphological processes, I argue that they exhibit anti-faithfulness effects (cf. Alderete 1999) and show that regular faithfulness and markedness constraints are not sufficient to explain them in OT. I develop a model in which RM plays an essential role. Although RM has been used in various contexts in the earlier literature, I significantly expand the role of the constraint and provide a formal definition. I argue that interactions of RM and faithfulness constraints are of central importance in understanding realizational morphology. Furthermore, I discuss antifaithfulness theory (Alderete 1999) as an alternative possibility. I will claim that it is not a viable possibility for various empirical and conceptual reasons. 
Chapter 3 is devoted to the implementation of RTM in truncatory morphology. Natural languages exhibit two types of morphological truncation: subtractive morphology and templatic truncation. The most significant difference is what remains constant. In subtractive morphology, what is deleted is constant, and therefore, the phonological size of the residue varies depending of the length of the base, but the residue remains prosodically constant in templatic truncation. I will claim that both types of truncation are governed by the same underlying principle (i.e., RM » Max). The only difference is whether Max is dominated by a set of prosodic constraints restricting the phonological size of the entire output. If such constraints exist, templatic truncation emerges while subtractive morphology obtains otherwise. Concerning subtractive morphology, I examine a number of examples which have received various different analyses in derivational and constraint-based terms. I demonstrate that they are all explained by the same ranking schema (i.e., RM » Max) and discuss its superiority to earlier approaches.

Chapter 4 deals with multiple nonconcatenative allomorphs, cases where allomorphs are nonconcatenative rather than affixational. I maintain that they are governed by the same driving force as affixational allomorphs. The plural morpheme $-s$ in English, for instance, takes three allomorphs, but their distribution is regulated by phonological conditions. In OT terms, this is because some relevant markedness constraint is ranked over relevant faithfulness constraints. I propose that the same holds of nonconcatenative allomorphs: the choice of a particular nonconcatenative allomorph is languages' effort to optimize the overall harmony of the word, decreasing phonological markedness. However, the phonological environment to be considered is more global than affixational allomorphs in the sense that the overall 
word shape affects the selection of nonconcatenative allomorphs. This is not true of the allomorphemic alternation of the plural morpheme $-s$, since only the stem-final segment is pertinent.

Chapter 5 is devoted to the investigation of cases where a single morpheme enjoys two phonological exponents (i.e., double morphemic exponence). More specifically, I address why and how some stem modification (e.g., umlaut) is required in addition to affixation given the crosslinguistic fact that affixation is frequently enough to denote the concept of the idea of a morpheme in the surface representation. I propose that sympathy theory plays a pivotal role in this context and develop the idea that I call morphological opacity. The idea is that the affix contained in the underlying representation is made invisible for the purpose of satisfying RM through Stem $\equiv \operatorname{PrWd}$ (the stem domain must coincide with a prosodic word domain) that serves as the selector constraint. The net effect is that the whole affixational category is made opaque. The sympathy candidate is the one which undergoes some stem modification under duress of RM. The stem modificational property is inherited by the ultimate output form through a relevant Faithis required by high ranked Max-IO, obtaining two phonological exponents for a single morpheme.

Throughout these chapters, RM plays a pivotal role. Finally, this dissertation is closed by a brief summary of the main results of the investigation carried out in this dissertation (Chapter 6). 


\section{Chapter 2}

\section{Deriving Nonconcatenative Morphology}

\subsection{Introduction}

The main goal of this chapter is to examine various formal properties of nonconcatenative morphology and to build up a theoretical system dubbed here "Realizational Morphology Theory (RMT)". RMT offers a comprehensive and explanatory account for the range of morphological phenomena of relevance. The type of root-and-pattern morphology attested in Semitic languages is a famous example of nonconcatenative morphology, where phonological segments of more than one morpheme are dispersed and intermingled such that a unique demarcation of the morphemes cannot be drawn. The focus of the nonconcatenative morphological processes here is not on root-and-pattern or templatic morphology, however. Rather, nonconcatenative morphology is roughly defined here as morphologically conditioned phonological changes without fixed segmentism. Thus, additive or concatenative morphology like the plural formation in English (e.g., [kæt-s $]_{\text {Plural }}$ ) is outside the scope of principal interest. The phenomena considered instead include subtractive morphology, morphologically governed metathesis, various segmental changes known as umlaut, suppletion, and mutation, morphological haplology or fusion, among others. They are clearly nonconcatenative since a stem undergoes some phonological modification to express the existence of a morpheme (e.g., part of a stem is elided in subtractive morphology). Another important phenomenon to be

considered below is reduplication. Reduplication falls into the category of phenomena investigated here since the reduplicant does not have fixed segmental 
content, although reduplication is standardly regarded as a kind of concatenative morphology like regular affixation (Marantz 1982).

It is nonetheless quite important to pay close attention to concatenative morphology as well because concatenative and nonconcatenative morphology should both be understood as phonological manifestations to achieve the same goal, namely to obtain some overt phonological expression to illuminate the presence of a morpheme. Thus, uncovering underlying formal similarities and differences between concatenative and nonconcatenative morphology is of critical importance in a unified and principled understanding of the two types of morphology. In the subsequent sections of this chapter, therefore, the two types of morphology will be compared when necessary.

The rest of this chapter is devoted to a careful examination of fundamental descriptive aspects of nonconcatenative morphology, followed by a proposal of a general theory which provides a satisfactory account in a unified manner. In section 2.2, I investigate the nature of nonconcatenative morphology and claim that it exhibits anti-faithfulness effects which should be crucially distinguished from non-faithfulness effects observed in regular phonology. As will be argued in detail, this is the most outstanding property of nonconcatenative morphology distinguishing it from concatenative morphology. In section 2.3 , I examine the role played by a Realize Morpheme constraint (RM) proposed and employed in various contexts in the earlier literature such as Samek-Lodovici (1993), Akinlabi (1996), Gnanadesikan (1997), Rose (1997), Walker (1998, 2000), Piggott (2000), and Kurisu (1999, 2000ab, 2001, to appear) among others. Paying close attention to similarities and differences between concatenative and nonconcatenative morphology, the notion of RM is 
conceptualized and formalized toward an integrated understanding of realizational morphology. This is a key constraint throughout this dissertation and plays a central role in the analysis of various nonconcatenative morphological phenomena. After establishing RM, I propose a general schema to account for nonconcatenative morphology in section 2.4. The essential notion is relativized faithfulness, the relativization referring to morphosyntactic categories. I also discuss some important theoretical consequences arising from the general theory in this section. Given antifaithfulness effects, an obvious analytical possibility is anti-faithfulness theory, as advocated by Alderete (1999). In section 2.5, I critically review anti-faithfulness theory and maintain that it is not an appropriate theoretical device to analyze the range of phenomena considered here. I also discuss that the theory itself is not desirable for various conceptual reasons. Arguments developed in this section are based on both empirical and conceptual grounds. Finally, section 2.6 summarizes the main results of this chapter.

\subsection{Anti-Faithfulness Effects}

This section is aimed at investigating the formal characteristics of nonconcatenative morphology under consideration. The most prominent common property shared by nonconcatenative morphological processes is that a base form is subject to a phonological change in some manner. In subtractive morphology, for instance, part of the base is subject to elision so that the form of the derived grammatical category is phonologically smaller than the base form. From the perspective of correspondence theory (McCarthy and Prince 1995), this incurs one or more violations of Max which requires each input segment to have a correspondent in the output representation. In 
the same fashion, various kinds of faithfulness constraints are violated in the whole range of nonconcatenative morphology, as summarized in (1).

\begin{tabular}{|c|c|}
\hline Morphological Process & Violated Constraint \\
\hline Subtractive morphology & Max \\
\hline Umlaut, Suppletion, Mutation & Ident \\
\hline Morphological epenthesis & Dep \\
\hline Metathesis & Linearity \\
\hline Infixation & Contiguity \\
\hline Reduplication & Integrity \\
\hline Haplology (Fusion) & Uniformity \\
\hline
\end{tabular}

Faithfulness violations are frequently observed in regular phonology, but the nature of faithfulness violations involved in nonconcatenative morphology is special. As a simple example of phonological phenomena where a faithfulness violation occurs, consider word-final devoicing in German. As exemplified in (2), German exhibits a voicing alternation in the word-final position. The descriptive generalization is that word-final obstruents are devoiced word-finally (Rubach 1990; Wiese 1996b). The fact is more complicated than this oversimplified generalization, but the precise formulation does not concern us here. Assuming WFD as a descriptive constraint motivating word-final devoicing in the relevant environment, the alternating pattern can be easily captured by WFD»Ident-IO-[voi]. The crucial point is that the devoicing process is phonologically governed in the sense that all that matters is the phonologically defined position occupied by the relevant obstruent. Thus, phonologically motivated changes are explained by Markedness » Faithfulness. 
(2)

Voiced forms

Gloss

Devoiced forms

Gloss

$\begin{array}{llll}\text { Kind }[\mathrm{t}] & \text { child } & \text { Kind-isch }[\mathrm{d}] & \text { childish } \\ \text { Tag }[\mathrm{k}] & \text { day } & \text { Tag-e }[\mathrm{g}] & \text { days } \\ \text { Haus }[\mathrm{s}] & \text { house } & \text { Häus-er }[\mathrm{z}] & \text { houses }\end{array}$

Next, consider the data given in (3).

$\begin{array}{llll}\text { Infinitive } & \text { Gloss } & \text { Deverbal Noun } & \text { Gloss } \\ \text { klifra } & \text { climb } & \text { klifr } & \text { climbing } \\ \text { kumra } & \text { bleat } & \text { kumr } & \text { bleating } \\ \text { grenja } & \text { cry } & \text { grenj } & \text { crying } \\ \text { söötra } & \text { sip } & \text { söötr } & \text { sipping } \\ \text { puukra } & \text { conceal } & \text { puukr } & \text { concealment } \\ \text { kjöökra } & \text { wail } & \text { kjöökr } & \text { wailing }\end{array}$

As the examples show, deverbal nouns are derived from the corresponding infinitive forms in Icelandic. The generalization is that a word-final vowel is deleted in the deverbal noun formation (Orešnik 1972, 1978ab; Arnason 1980; Kiparsky 1984; Itô 1986; Benua 1995). Since vowel deletion takes place, one might postulate a markedness constraint such as Free- $\mathrm{V}$ that militates against the presence of a wordfinal vowel (Prince and Smolensky 1993) or Final-C which requires a word to be closed by a consonant (McCarthy 1993). However, if the constraint is active in Icelandic phonology in general, we do not expect the presence of a final vowel in infinitive forms either, whatever the markedness constraint is. This suggests that final vowel deletion observed in the deverbal noun formation is not phonologically conditioned. In other words, there exists no phonological markedness constraint that motivates vowel deletion in the deverbal noun formation. More interestingly, deverbal nouns are in a sense more marked than infinitive forms because *Complex(coda) is violated in the former while it is otherwise completely satisfied in Icelandic in general. Moreover, the distribution of long vowels is restricted to 
stressed open syllables elsewhere in Icelandic. Thus, the general ranking schema holding of phonologically conditioned changes (i.e., Markedness » Faithfulness) cannot be extended to subtractive morphology. Rather, the reason why faithfulness violations are incurred is morphological. More specifically, they are required to express the presence of a morpheme overtly in the surface representation. Thus, the overt phonological contrast between infinitives and deverbal nouns is denoted by the presence/absence of a final vowel in Icelandic, for instance. The same is true of other nonconcatenative morphological operations such as morphological metathesis and umlaut and so on listed in (1). Given this argumentation, I call faithfulness violations involved in nonconcatenative morphology anti-faithfulness effects following Alderete (1999) in order to make a clear distinction between faithfulness violations in regular phonology and those in nonconcatenative morphology.

The reason why nonconcatenative morphology is an interesting area in the context of the OT program becomes even clearer if we also pay attention to the fact that derived forms have worse faithfulness values compared with a perfectly faithful candidate. This is obvious because deverbal nouns decrease their faithfulness by clipping a final vowel in Icelandic, for example. This indicates that neither the Markedness » Faithfulness nor the Faithfulness » Markedness schema is satisfactory for capturing various nonconcatenative morphology. The immediate question is then what serves as the driving force of stem modifications given the OT assumption that the set of universal constraints, Con, consists only of faithfulness and markedness constraints. The answer to this question is postponed until sections 2.3 and 2.4.

This problem does not arise in concatenative morphology employing normal affixation because the phonological shape of a base remains unaffected. Even if the 
stem form undergoes some morphophonological change by fulfilling the environment in which some phonological change takes place on the stem (e.g., regressive place/voicing assimilation), it is a matter of phonology rather than morphology. Such cases are simply explained by ranking an appropriate markedness constraint over competing faithfulness constraints (see chapter 5 for cases where a stem change takes place in addition to affixation for purely morphological reasons). This difference between concatenative and nonconcatenative morphology resides in their more fundamental difference. While the morpheme attached to a stem has some phonological material in concatenative morphology, the morpheme yielding a derived category does not contain any such element in nonconcatenative morphology.

Before closing this section, some remarks are in order regarding Dep, Contiguity, and Integrity in (1). The first point concerns Dep. That Dep is violated represents the kind of morphology where some (maybe default) segment is epenthesized to denote a particular grammatical category. One might claim that such a case is actually not attested since the default segment would be reanalyzed as an authentic affix associated with the relevant morphosyntactic category. Presumably, the only one potential argument for this claim would come from the consideration of lexicon optimization (Prince and Smolensky 1993:192; see also Itô, Mester and Padgett 1995 and Kurisu 2000c). The tenet of lexicon optimization is that the most harmonic input to the output should be selected as the real input. With this notion, the analysis assuming that the relevant morphosyntactic category is phonologically contentless is clearly eliminated due to its excessive violation of Dep. But this is not a valid argument. Behind lexicon optimization, richness of the base exists as a more fundamental principle (Prince and Smolensky 1993; Smolensky 1996; Kurisu 2000c), 
the principle which is also known as freedom of the input. OT is output-oriented such that the system does not allow for restrictions on inputs. Lexicon optimization is merely a strategy to make the lexical organization simpler at some point of the language acquisition process, but crucially, it does not preclude the possibility of maintaining richness of the base. Nothing thus excludes languages from employing morphological epenthesis. Indeed, as will be discussed in section 4.3, Upriver Halkomelem does take advantage of schwa epenthesis to denote the continuative aspect under a certain circumstance.

This argumentation contradicts with Consistency of Exponence (McCarthy and Prince 1993b:20-21). Consistency of Exponence maintains that no changes in the exponence of a phonologically-specified morpheme are permitted, and in particular, that epenthetic segments posited by Gen do not have any morphological affiliation under any circumstances. According to McCarthy and Prince, this principle is underlying as a universal property of Gen, and therefore, Gen does not produce any candidate which infringes on Consistency of Exponence. However, given cases where epenthesis of a default segment denotes some morphosyntactic information, Consistency of Exponence is empirically invalidated.

Second, infixation is clearly one type of affixations but can be subsumed under the family of nonconcatenative morphology from the perspective that it incurs a faithfulness violation. A single stem is split up into two chunks in infixation, resulting in a violation of Contiguity. Although this is one possible way of grouping infixation in the class of nonconcatenative morphology, it is not clear whether this categorization has any empirical and/or theoretical consequences. There is no literal prefix, infix, or suffix in OT. Rather, the exact position of an affix is determined by 
independent constraints such as alignment. As for infixation, it is unlikely that infixation is required solely for morphological reasons. In Tagalog and Toba Batak, the verbal affix um manifests itself either as a prefix or as an infix depending on the phonological shape of the stem it is attached to: $u m$ is prefixed when a stem begins with a vowel (e.g., um-akyat 'teach') whereas it is infixed right after the first onset if the stem begins with a consonant (cluster) (e.g., k-um-uha 'get') (see Blake 1925; Alejándro 1963; Ramos 1974; French 1988; McCarthy and Prince 1993ab; Prince and Smolensky 1993 for Tagalog, and Nababan 1981; Percival 1981; Crowhurst 1998 for Toba Batak). It might be hasty to draw a definite conclusion from these two languages, but the same pattern is widespread across Malayo-Polynesian languages such as Chamorro (Topping 1973), Yogad (Davis, Baker, Spitz and Baenk 1996), and Ilokano (Davis 1995), so it is highly probable that infixation is a reflex of some phonological influence that forces an alignment infringement, as argued by Prince and Smolensky (1993) and McCarthy and Prince (1993ab). This indicates that purely morphological infixation is unlikely to exist, casting a suspicion that the reason why infixation is nonconcatenative is phonological. Whether this generalization is true or not, the following argument is not affected since the proposal made later in section 2.3 covers both concatenative and nonconcatenative morphology in an integrated manner.

Finally, I assume that each reduplicative segment incurs an Integrity violation. McCarthy and Prince (1995) and many subsequent works on reduplication assume that reduplicated segments are exempted from IO-faithfulness constraint violations. Given the fact that the concrete segments are absent from the input specification of the reduplicant, one might maintain that a reduplicated segment would be charged a 
Dep violation. But creation of reduplicated segments is quite different in nature from phonologically motivated epenthesis. The crucial observation is that regular epenthesis is conditioned by phonological factors (e.g., syllable structure optimization). Thus, an epenthesized element is totally unrelated to other input/output segments in light of correspondence. Spaelti (1997:72) proposes a reduplication model in which the underlying form is in correspondence with both the reduplicant and the base, that is, with the whole reduplication form, as in (4).

The Reduplicate! model of correspondence (Spaelti 1997:72)

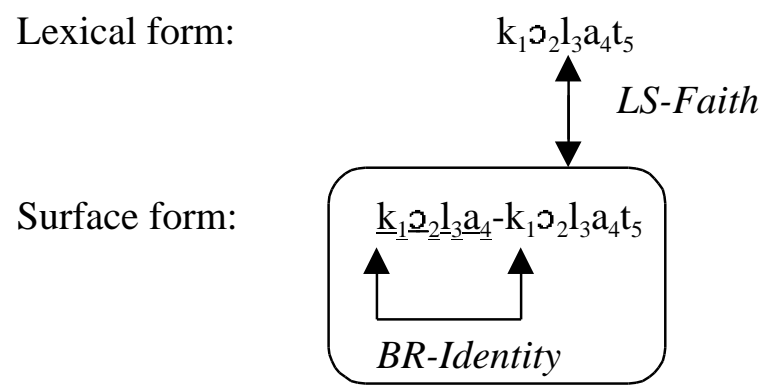

Within this model, both the reduplicant and the base are realizing segments in the underlying representation, so there are two chances to realize an underlying segment. Spaelti argues that multiple exponence of a single underlying element violates biuniqueness, or more accurately, Integrity which militates against a configuration in which a single input element stands in a correspondence relation with more than one output element tokenwise (McCarthy and Prince 1995:372). Following his argument, I assume that an Integrity violation is invoked by each reduplicated segment (see also Buckley 1998 and Struijke 1998 for other works that make the same assumption). Considered this way, reduplication also exhibits the same character as other obvious nonconcatenative morphological processes such as subtractive morphology, namely faithfulness violations. 


\subsection{Characterizing "Realize Morpheme"}

In this section, I explore the nature of the Realize Morpheme constraint (RM) and argue that it is the key constraint to account for various nonconcatenative morphological processes as well as concatenative morphology. Hence, it plays a central role in RMT proposed here. Versions of RM have been proposed and employed in a variety of contexts with different names in the earlier literature, including Samek-Lodovici (1993), Akinlabi (1996), Gnanadesikan (1997), Rose (1997), Walker (1998, 2000), Piggott (2000) and Kurisu (1999, 2000ab, 2001, to appear). Although the focus of this work is on nonconcatenative morphology, close attention is paid to concatenative morphology too to achieve a comprehensive understanding of morpheme realization.

$\mathrm{RM}$ is descriptively defined as a constraint which requires every underlying morpheme to receive some phonological exponence. Since the only explicit and visible way of morpheme manifestation is phonological, the precise formalization of RM must be doubtlessly cast from a phonological perspective. In the various works, the role of RM has been considered in the context of morphological gemination, featural morpheme expression, reduplication and so on. The common property of these phenomena is that some visible phonological element appears on the surface. They are in this sense akin to affixational word formations. In these cases, RM can be understood as a function mapping each morpheme onto some phonological substance with which it is affiliated (cf. Walker 2000).

A serious problem of the definition based upon a mapping between a morpheme and visible phonological content is that it is not sufficient for nonconcatenative morphological operations considered here. Consider subtractive 
morphology, for instance, where part of the base is deleted to obtain a derived morphosyntactic category. No visible phonological substance exists in such cases, and RM would be violated. Morphemes are frequently expressed nonconcatenatively without any phonological material independent of the base, so we need to reexamine the formal definition of RM.

In order to achieve a satisfactory understanding of RM, it is an essential question at this point to address what morphology is for. A simple but non-trivial answer is that morphology maintains contrasts among various morphosyntactic functions (Bloomfield 1933; Bat-El 2000). Suppose that morphemes are atomic morphological units that motivate various morphological contrasts. Given the definition of RM that every single morpheme needs to enjoy some overt phonological exponence and given morphemes as primitive units of morphology, it follows that $\mathrm{RM}$ is satisfied iff the outcome has some phonological property (but not necessarily phonological substance) which distinguishes it from the base form.

The existence of the newly introduced morpheme is denoted by affixal segments in concatenative morphology because the affix promises the phonological non-identity between the base and the derived forms as in the plural formation in English (e.g., [kæt] $\approx$ kæts]). On the other hand, in nonconcatenative morphology such as subtractive morphology and morphological metathesis, the absence and segmental reversal of some underlying element convey the presence of the given morphosyntactic information respectively (see (3) for subtractive morphology). Thus, independent phonological material does not always exist as the expression of a morphosyntactic function. Despite this difference in the surface implementation of morphological expressions, concatenative and nonconcatenative morphology share 
the same spirit. Given this consideration, RM can be now formulated as in (5). It is worth noting that $\beta$ is essentially associated with a morphosyntactic category but $\alpha$ does not have to be so. This point will be justified shortly below.

Realize Morpheme (RM):

Let $\alpha$ be a morphological form, $\beta$ be a morphosyntactic category, and $\mathrm{F}(\alpha)$ be the phonological form from which $F(\alpha+\beta)$ is derived to express a morphosyntactic category $\beta$. Then $\mathrm{RM}$ is satisfied with respect to $\beta$ iff $\mathrm{F}(\alpha+\beta) \neq \mathrm{F}(\alpha)$ phonologically.

In the deverbal noun formation in Icelandic in (6) (repeated from (3)), infinitives and deverbal nouns correspond to $\alpha$ and $\beta$ respectively since infinitives serve as the bases of corresponding deverbal noun forms. [klifra $]_{\text {Inf. }}$ and $[\mathrm{klifr}]_{\mathrm{DVN}}$ are phonologically non-identical by virtue of the fact that the deverbal noun form lacks the final segment [a] contained in the infinitive form. This means that $[\mathrm{klifr}]_{\mathrm{DVN}}$ satisfies RM with respect to the deverbal noun morpheme. By contrast, an incorrect deverbal noun form $*[\mathrm{klifra}]_{\mathrm{DVN}}$, which would be a plausible candidate produced by Gen, violates RM since this form is phonologically identical to [klifra $]_{\text {Inf. }}$. This is an instance of morphological elision, but phonological non-identity can be accomplished in various other ways. In other words, RM is satisfied as long as the candidate is not perfectly faithful to the form with which it is compared, where phonological nonidentity is exactly what represents morphological contrasts (cf. works on Dispersion Theory such as Flemming 1995 and Padgett 2001).

$\begin{array}{llll}\text { Infinitive } & \text { Gloss } & \text { Deverbal Noun } & \text { Gloss } \\ & & & \\ \text { klifra } & \text { climb } & \text { klifr } & \text { climbing } \\ \text { kumra } & \text { bleat } & \text { kumr } & \text { bleating } \\ \text { grenja } & \text { cry } & \text { grenj } & \text { crying } \\ \text { söötra } & \text { sip } & \text { söötr } & \text { sipping } \\ \text { puukra } & \text { conceal } & \text { puukr } & \text { concealment } \\ \text { kjöökra } & \text { wail } & \text { kjöökr } & \text { wailing }\end{array}$


The formulation in (5) is not yet satisfactory, however. An important question is what serves as the input of a morphosyntactic category. In the Icelandic case above, there is strong evidence that deverbal nouns are derived from infinitives (see section 3.2.3), but it is often the case that there is no such phonological evidence. It is frequently assumed with no justification that singulars are more basic than plurals, and therefore, that singulars serve as the inputs of plurals. But there are cases which do not receive a straightforward descriptive generalization under such an idea. Singular-plural pairs in Aka, a Bantu language spoken in Central African Republic, are good examples. As exemplified in $(7 \mathrm{a}, \mathrm{b})$, where tonal specifications are omitted, the feature [voice] is the singular (class 5) marker whereas the plural (class 6) is denoted by the prefix $m a$ - (Akinlabi 1996:285-286). Thus, if the initial consonant of a stem is voiceless, it is voiced in the singular formation (7a) while no voicing change takes place if the stem-initial consonant is originally voiced (7b). This generalization is confirmed by the fact that class 9 singular forms do not exhibit the stem-initial voicing alternation but their plural forms (class 6) still take the prefix $m a-$. Since the plural morpheme is manifested by the prefix $m a-$, no voicing change is involved, as can be seen from the plural forms in (7c).

$$
\text { Singular(Class 5) Plural (Class 6) Gloss }
$$

a.

denge
dots
gasa
gini
boki
bapulaka
Bondu
Boko

b. goala

belele dz̧amba

ma-tenge
ma-toto
ma-kasa
ma-kini
ma-poki
ma-papulaka
ma-фondu
ma-фoko

ma-goala ma-belele ma-dzamba piercing tool cartridge palm branch fly arch of the eyebrows lung goiter hole game of imitation sound of a waterfall mud 


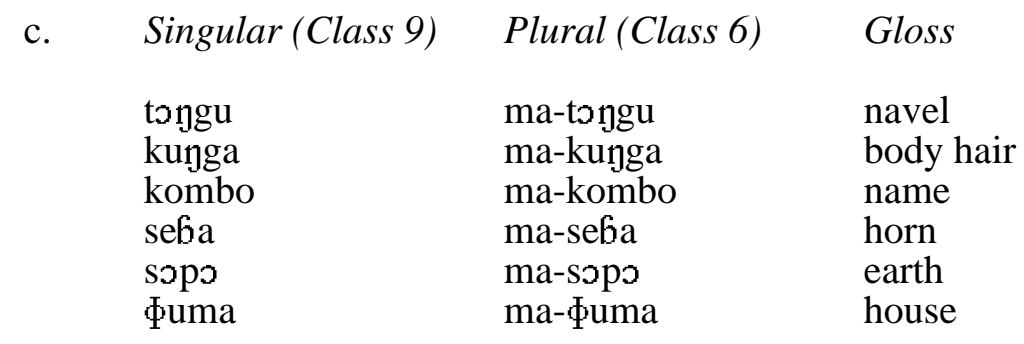

The singular forms cannot be assumed as the underlying representations which serve as the bases of their plural counterparts. Were the plural forms derived from the singular forms, stem-initial devoicing must be posited in (7a), but this does not account for the plural examples in (7b) where the stem-initial consonants remain voiced. Moreover, this kind of devoicing process is not motivated at all both language-internally and crosslinguistically. Rather (7) can be straightforwardly accounted for by hypothesizing that the bare stems (i.e., plural forms minus the prefix $m a-)$ are the inputs to the singular and the plural forms. Voicing of the initial consonant of a bare stem characterizes the presence of the singular morpheme while prefixation of $m a$ - signals the plural morpheme. The important lesson is that the normally assumed basic-derived notion does not universally hold. Many cases where the singular serves as the basis of the plural do not give rise to any descriptive discrepancy are examples where singular forms incidentally coincide with stems. Given the Aka case, I claim that stems lack morphosyntactic information. They consist only of phonological, syntactic, and semantic information.

Given bare stems as inputs for various morphosyntactic categories, is it possible to compare those bare stems with output candidates for the purpose of RM evaluations? The answer is negative. In tandem with the output orientation of the theory, OT maintains richness of the base as a fundamental slogan, which grants any freedom to the input (Prince and Smolensky 1993; Smolensky 1996; Kurisu 2000c). 
It is therefore impossible to restrict the phonological representation of the input to a unique form. This in turn suggests that the output candidates do not reliably possess a single phonological input form with which they are compared to compute the satisfaction/violation of RM. Only an output reliably has a fixed phonological representation. This indicates that we need to establish an output-output mapping strategy for the calculation of RM violations. My proposal is that the grammar evaluates the optimal form of the bare stem on the basis of the constraint hierarchy independently motivated by the phonology of the language. The bare stem output is therefore not necessarily an actual surface form in the language. Output candidates bearing a morphosyntactic category are then compared with it, as in (8).

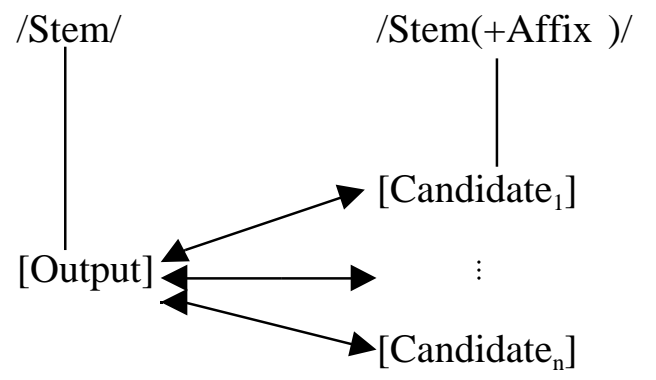

Given this conceptualization, RM is satisfied if the candidate is non-identical to the output of the bare stem whereas the constraint is violated if they have exactly the same phonological representations, as schematically exemplified in (9). The nonidentity required for the satisfaction of $\mathrm{RM}$ is not confined to any specific phonological deviance from the base. As will be discussed in the next section, the specific phonological instantiation of morpheme realization is determined by the faithfulness constraint ranked below RM, and therefore, RM itself does not require any particular phonological realization of a morpheme. 
(9)

\begin{tabular}{|c|c|c|c|c|}
\hline & Bare stem output & Candidate & $\mathrm{RM}$ & Remark \\
\hline a. & {$[\mathrm{ABC}]$} & {$[\mathrm{ABC}]_{\alpha}$} & violated & no change \\
\hline b. & {$[\mathrm{ABC}]$} & {$[\mathrm{ABCD}]_{\alpha}$} & satisfied & affixation \\
\hline c. & {$[\mathrm{ABC}]$} & {$[\mathrm{AB}]_{\alpha}$} & satisfied & deletion \\
\hline d. & {$[\mathrm{ABC}]$} & {$[\mathrm{ACB}]_{\alpha}$} & satisfied & metathesis \\
\hline
\end{tabular}

First, (9a) represents the case where RM is violated. Comparing the output of the bare stem and that of the derived form which carries some morphosyntactic category denoted by $\alpha$, they are exactly the same (i.e., [ABC]), as in (10a). This identity means that morpheme $\alpha$ attached to the bare stem does not receive any phonological exponence, resulting in a RM violation. Second, (9b) is concatenative morphology involving suffixation. As illustrated in (10b), the output of the bare stem does not contain any affix, but the output of the derived category bears a suffixal segment $D . \quad[\mathrm{ABC}]$ and $[\mathrm{ABCD}]_{\alpha}$ are phonologically different: $\mathrm{RM}$ is satisfied. Finally, (9c) and (9d) are subtractive morphology and morphological metathesis respectively. $\mathrm{RM}$ is fulfilled by eliding a segment $C(9 \mathrm{c})$ or by reversing the order of two segments (9d). As shown in (10c) and (10d), RM is satisfied in these cases.

a.

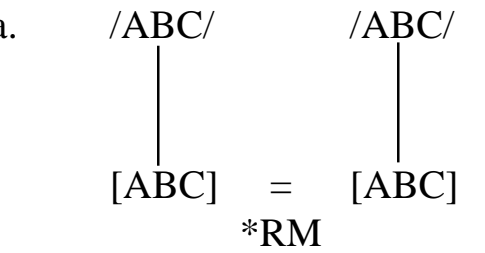

c.

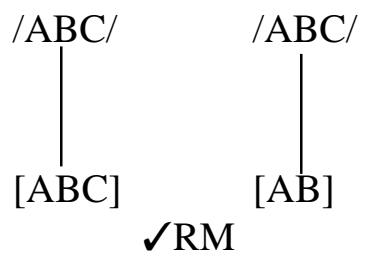

b.

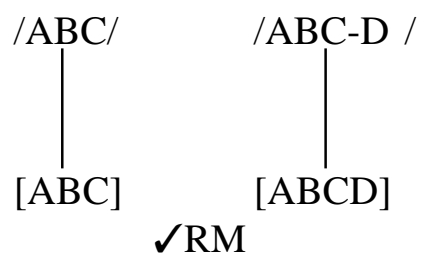

d.

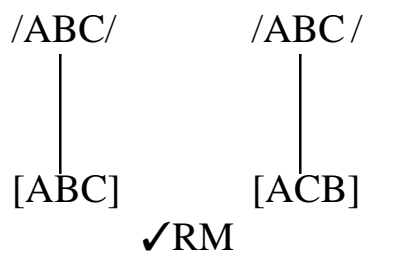


In the plural formation in English, for instance, plurals correspond to $\beta$ in (5), and the relevant bare stems (i.e., input to plural forms) correspond to $\alpha$ in (5). The affixal material /-s/ is associated with the plural morpheme such that $\left[\mathrm{k}^{\mathrm{h}} æ \mathrm{ts}\right]_{\text {Plural }}$ is phonologically non-identical to $\left[\mathrm{k}^{\mathrm{h}} æ \mathrm{t}\right]$, the output of the bare stem, as depicted in (11a). Under the proposed system of RM evaluations, singulars are derived from bare stems as well. As exemplified in (11b), however, $\left[\mathrm{k}^{\mathrm{h}} æ \mathrm{t}\right]_{\text {Singular }}$ is phonologically the same as the output of the bare form, and therefore, RM is violated. In other words, the singular morpheme does not receive any phonological exponence in English whereas plurals do. This point will be significant to the system developed in the next section. An important point to be emphasized here is that the output of a base stem does not have to be an actual output in the language. Rather, it is at least a possible output of the language, unlike the definition of the output in the sense of Benua (1997). This is important to differentiate RMT from anti-faithfulness theory, as will be discussed in section 2.5.
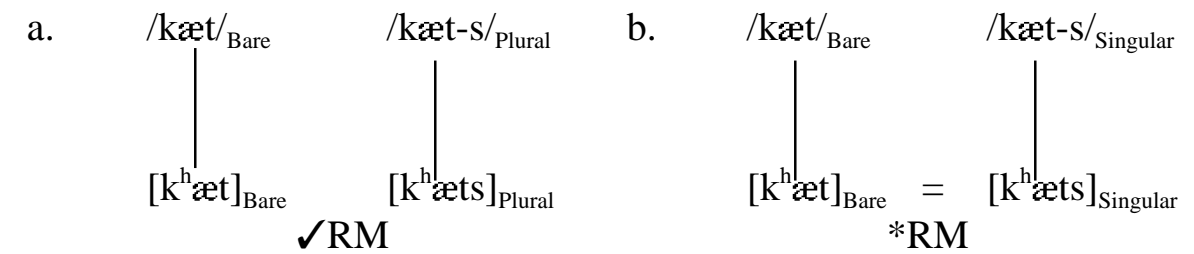

By contrast, RM directly compares the input and the output when the input is already an independent output form like Icelandic infinitives, as shown in (12). The direct input-output comparison for the purpose of calculating RM violations is possible because compared forms are independent output forms which reliably have a unique phonological representation. It is important to note here that the output of a bare stem does not play any role in the evaluation of RM violations in such cases. This is because bare stems play a role only when they serve as the inputs of a given 
morphosyntactic category (i.e., when the relevant morphosyntactic category is not derived from another full-fledged output form).

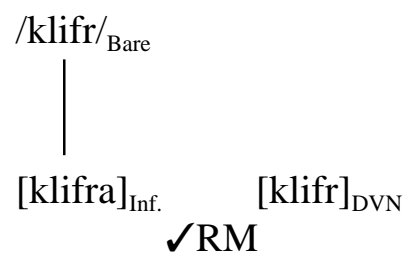

The notion of the bare stem output has a further extension. Itô and Mester (1998) discuss productive truncation in modern German. As given in (13), the output is a single syllable followed by the suffix $-i$ (Itô and Mester 1998:52).

a. Personal names

Base forms Truncation forms

$\begin{array}{ll}\text { Gàbriéle } & \text { Gábi } \\ \text { Wáldemar } & \text { Wáldi } \\ \text { Stéfanìe } & \text { Stéffi } \\ \text { Úlrich } & \text { Úlli } \\ \text { Ótto } & \text { Ótti }\end{array}$

b. Surnames

Base forms Truncation forms

Górbatschòw Górbi

Hónecker Hónni

Schimánsky Schímmi

Klínsmànn Klínsi

Schláppner Schláppi

c. Common nouns

$\begin{array}{lll}\text { Base forms } & \text { Truncation forms } & \text { Gloss } \\ & & \\ \text { Àlkohóliker } & \text { Álki } & \text { alcoholic } \\ \text { Ámerikáner } & \text { Ámi } & \text { American } \\ \text { Schátz } & \text { Schátzi } & \text { darling } \\ \text { Mútter } & \text { Mútti } & \text { mother } \\ \text { Pròletárier } & \text { Próli } & \text { proletarian }\end{array}$

The crucial observation here is that [gorb], for instance, is not a constituent of some input, actual surface form itself, constituent of the base form (cf. [gor.ba.t $\int_{\text {of] }}$ ), 
or constituent of the truncation form (cf. [gor.bi] vs. *[gorb.i]). In effect, [gorb] is not a constituent at any level of representation. Itô and Mester (1998) claim that the single syllable found in truncation is rather a maximal possible syllable in German. They employ sympathy theory (McCarthy 1999) such that the maximal possible syllable is selected as the sympathy candidate and that the non-surface constituent becomes visible to the evaluation of the eventual output.

Given the notion of bare stem output, another way of considering the relevant examples is to regard the truncated stem forms as possible stems in German. Calling them truncatory stems, they are potentially available for morphological operations. For the case at hand, hypocoristic formation, for instance, is relevant. Given the eligibility of [gorb] as a possible stem in German, then the hypocoristic formation seen in $(13 a, b)$ is simply an issue of suffixation. This idea and the notion of the bare stem output share the property that some kind of non-surface forms plays a central role in natural languages.

Summarizing thus far, RM requires every input morpheme to receive some phonological exponence. The satisfaction or violation of RM is based on outputoutput comparisons. Output candidates yielded by Gen are compared with the output form of a bare stem when the bare stem serves as the input, but RM compares output candidates and the input when the input is already an output form which carries some morphosyntactic information.

The formalization of RM in terms of output-output comparison has a clear advantage besides the theoretical need in the framework of OT. Consider the wordfinal devoicing process in German illustrated in (2). As aforementioned, this is an automatic phonological process which takes place across the board regardless of 
morphological conditions. Given the assumption that bare stems are free from morphosyntactic categories, they serve as the inputs of singular forms. Given the voicing alternation shown in (2), where a final obstruent is realized as a voiced segment when followed by a vowel-initial suffix, the final segment should be voiced in the underlying representation. Obviously, the singular outcome is phonologically non-identical to the input (of the bare stem) due to word-final devoicing, but the question is whether this voicing change counts as valid to satisfy RM. The answer is negative. RM needs to be satisfied for morphological reasons, but the devoicing process has nothing to do with morphology. But how can RM distinguish morphologically induced phonological changes from purely phonological ones? There is no principled answer to this because RM merely requires a phonological distinction between two forms. But if the output of the bare stem is computed against the constraint ranking independently needed in the phonology of German (i.e., WFD » Ident-IO-[voi]), the optimal bare stem form should carry a voiceless obstruent word-finally (e.g., [kmt] 'child'). The candidates produced by Gen are compared with this form for the purpose of evaluating the satisfaction/violation of RM. The optimal singular form derived from $/ \mathrm{Kmnd} /$ Singular $\left(\right.$ i.e., $[\mathrm{kmnt}]_{\text {Singular }}$ ) is phonologically identical to the optimal bare stem form, resulting in a violation of RM, as illustrated in (14). Thus, the calculation of the best form of a bare stem also enables the computation of the RM satisfaction/violation to be purely morphologically grounded.

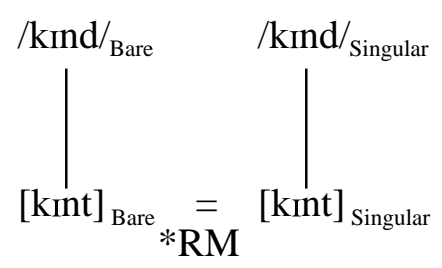


An important theoretical implication of the conceptualization of RM here is that zero morphs entirely lose their place in the system of morphology. Given productive $s$-suffixation in the plural formation in English, for instance, it has been assumed quite extensively that plural forms without any singular-plural distinction actually contains an invisible empty suffix $-\varnothing$ (e.g., $[\mathrm{fI} f]_{\text {Singular }} \approx[\mathrm{fI} f-\varnothing]_{\text {Plural }}$ 'fish'). Under RMT developed here, RM is satisfied if and only if some overt phonological difference is found, and thus, empty morphs have no room to play a role for the satisfaction of RM. The existence of zero morphs has been advanced on an analogical basis, but it would be nothing more than a theoretical artifact given the system where they do not contribute to morphological contrasts. Although the earlier literature often claims that morphological zero is categorially meaningful absence, how phonological absence can be computed in isolation forms (e.g., [fIf]) is totally unclear. The singular-plural pairs like fish can be unambiguously interpreted only when an appropriate context is given. Zero morphs carry no substantive role at the word level with which morphology is concerned. In addition, independent arguments have been developed against zero morphs on various empirical and conceptual grounds (cf. Sanders 1988; Pullum and Zwicky 1991). The total abolishment of zero morphs is presumably desirable in terms of transparency in language processing too.

Closely related to this first implication, another case where a RM violation is potentially incurred is morphological haplology or fusion, where the phonological element belonging to separate morphemes are fused on the surface (see Russell 1995, Plag 1998 and de Lacy 1999 for recent work on haplology within the framework of OT). A simple example is the cooccurrence of the plural and possessive morphemes in English (e.g., /kId-z-z/ 'kid-Pl.-Poss.'). Due to the OCP effect against the two 
adjacent identical obstruents, the ultimate output should be $[\mathrm{kidz}]_{\text {PI.Poss. }}$ rather than * $[\text { kIdzz }]_{\text {Pl.Poss. }}$ The most standard and widely accepted analysis is that the two homophonous morphemes are both phonologically realized as a single segment. Given the formulation of $\mathrm{RM}$ as defined here, $\mathrm{RM}$ is clearly violated by $[\mathrm{kIdz}]_{\text {PI.Poss }}$ since this output form is phonologically the same as the form without the possessive morpheme (or equivalently, the form consisting only of the bare stem and the plural morpheme), as in (15a). Dotted lines represent morpheme-phonology mapping. The reason why $\mathrm{RM}$ is violated here is that $\mathrm{RM}$ is strictly phonologically defined. Although $[\mathrm{kIdz}]_{\mathrm{Pl} \text {.Poss. }}$ is structurally different from $[\mathrm{kIdz}]_{\mathrm{Pl}}$ if the morphemephonology mapping is considered, it plays literally no role for the computation of RM violations. This point becomes clearer if we hypothesize another possibility that the phonological substance of the possessive morpheme (i.e., the second /z/) remains unparsed, as in (15b). The plural possessive form in (15b) violates RM in addition to Max, and the plural possessive form in (15a) is the same as that in (15b) as far as their phonological representations are concerned. The identity of the two plural possessive forms in (15a) and (15b) suggests a RM violation of $[\mathrm{kIdz}]_{\text {Pl.Poss. }}$ in (15a).

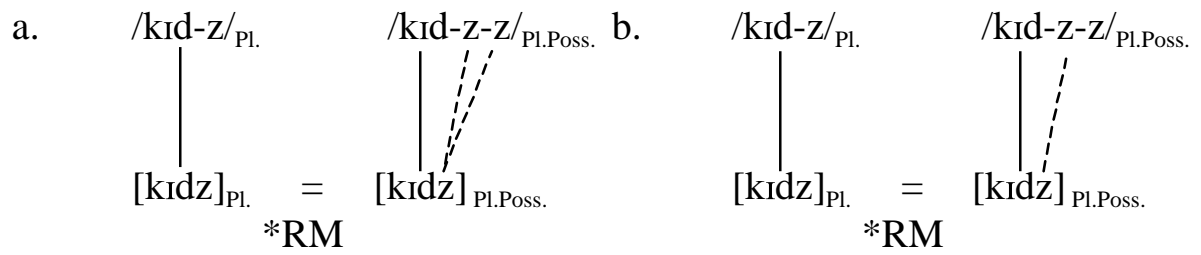

Note that haplology does not always fail to satisfy RM, however. Suppose that $/ \mathrm{CV}_{1} \mathrm{CV}_{2} /$ is given as a bare stem and that the output form of this is $\left[\mathrm{CV}_{1} \mathrm{CV}_{2}\right]$. Provided that morpheme $\alpha$ is attached to the bare stem to express some morphosyntactic function and that vowel haplology is employed as the specific 
strategy of morpheme realization, the outcome is $\left[\mathrm{CV}_{1,2} \mathrm{C}\right]$. In this case, $\left[\mathrm{CV}_{1,2} \mathrm{C}\right]$ is clearly phonologically different from $\left[\mathrm{CV}_{1} \mathrm{CV}_{2}\right]$ (e.g., the syllable count is different), and thus, RM is satisfied. Indeed, this state of affairs is actually attested, as will be discussed in section 4.5. Feature values are often affected even in those cases where haplology occurs between two adjacent segments: haplology does not necessarily invoke a violation of RM.

The strictly phonological orientation of the definition of RM has a further theoretical implication. Morphemes without any phonological content receive their phonological realization through some stem modification, but another possibility is merely positing the morpheme-phonology mapping of the kind demonstrated in (16) below, where no stem modification occurs but the morpheme $\alpha$ is affiliated with or parasitic on a stem segment. This strategy is in a sense more harmonic than any forms which undergo some phonological reshaping of the stem because no phonological faithfulness violation is incurred in (16). Furthermore, the candidate in (16) should also be better than those candidates with some stem modification in terms of phonological markedness since morphologically induced phonological processes normally create a phonologically more marked representation or structure, as discussed in section 2.2. Thus, the kind of morpheme-phonology mapping in (16) cannot be ruled out by faithfulness or markedness constraints. (16) is eliminated by $\mathrm{RM}$ instead because the output of the bare stem and that of morphosyntactic category $\alpha$ are phonologically identical.

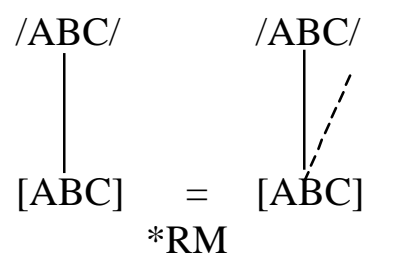


Comparing $[\mathrm{ABC}]_{\alpha}$ with the output of the stem (i.e., $[\mathrm{ABC}]$ ), they are precisely the same phonologically, however. Under the formalization of RM here, $\mathrm{RM}$ is violated by $[\mathrm{ABC}]_{\alpha}$. Whether the input of the bare stem surfaces faithfully in the output or not does not impinge on the argument here. Even if the output of the bare stem is unfaithful to the input, it is purely for some phonological factor as in the word-final devoicing process in German. Since no affixation is involved in $/ \mathrm{ABC}_{\alpha}$, $[\mathrm{ABC}]_{\alpha}$ should be subject to the same phonological change as the bare stem. Since what matters here is the identity between the outputs of $/ \mathrm{ABC} /$ and $/ \mathrm{ABC}_{\alpha}$, this faithfulness issue is beside the point. This point becomes significant in connection with Consistency of Exponence (McCarthy and Prince 1993b:20-21). It prohibits a phonologically specified morpheme from changing its exponence. Given that RM evaluates the (non-)identity between $[\mathrm{ABC}]_{\alpha}$ and the output of the bare stem (i.e., $[\mathrm{ABC}]),[\mathrm{ABC}]_{\alpha}$ in (16) should fulfill Consistency of Exponence since bare stems are free from any morphosyntactic categories. This means that this kind of candidates can be generated even if Consistency of Exponence is posited as a universal property of Gen, and thus, the role played by RM here is essential.

As discussed in chapter 1 , the issue of uniform conceptualization of overall word formation was a matter of controversy among structural linguists. As would be clear from the discussion thus far, one assumption made here is that there exist two types of morphemes: those with inherent phonological substance (as in affixation) and those without it (as in nonconcatenative morphology such as subtractive morphology). Put differently, affixation is treated as Item-and-Arrangement while stem changes involved in nonconcatenative morphology are more like Item-andProcess. The mixture of Item-and-Arrangement and Item-and-Process here deserves 
some discussion in the context of OT. Given empirical problems that the Item-andArrangement model encounters with respect to nonconcatenative morphology such as root-and-pattern morphology and subtractive morphology, Item-and-Process is strongly supported by many current morphologists. The question here is thus whether the position admitting morphemes as entities is valid or not. Russell $(1995,1999)$ addresses this question, bringing up three potential possibilities: morphemes as representations, morphemes as rules, and morphemes as constraints. The first possibility corresponds to Item-and-Arrangement, regarding morphemes as substantive entities. The other two are derivation-based and constraint-based versions of Item-and-Process respectively. Given the constraint-based theoretical architecture of OT, the position taking morphemes as rules can be ignored here. Comparing morphemes as representations and morphemes as constraints, Russell (1995, 1999) argues for the latter, pushing the Item-and-Process view to the extreme. Eventually, he proposes a model without Gen, following Bird (1990), Scobbie (1991), and Russell (1993) (see also Hammond 1995).

Besides cases where a morpheme does not carry fixed segmentism (i.e., reduplication, truncation, subtractive morphology, metathesis etc.), Russell raises a duplication problem as a serious pitfall of the position viewing morphemes as representations. For example, in Ulwa, a language spoken in Nicaragua, the possessive affix $k a$ is suffixed to the head foot of the word, as illustrated in (17) (McCarthy and Prince 1990, 1993ab, originally due to Bromberger and Halle 1988 and Hale and Blanco 1989). The essential point of Russell is that, under the model viewing morphemes as representations (or Item-and-Arrangement), encoding the affix $k a$ in the lexicon is not sufficient since the precise position of the affix is left 
undetermined. In effect, Ulwa needs to posit a constraint like Align $\left([\mathrm{ka}]_{\mathrm{Af}}, \mathrm{L}, \mathrm{Ft}, \mathrm{R}\right)$ as done by McCarthy and Prince (1993a:80). Given this alignment constraint, however, the affix encoded in the lexicon seems unnecessary because the affixal information is encoded in the alignment constraint anyway. Expanding the theory of generalized alignment (McCarthy and Prince 1993a), Russell $(1995,1999)$ advances the idea to eliminate the lexical information, proposing that Align(Affix,Edge,Stem,Edge) makes an exclusive Item-and-Process model possible in the framework of OT. It is crucially important to remark here that any affixation incurs one or more Dep violations since it involves insertion of phonological segments that do not exist underlyingly.

$$
\text { Base Possessive Gloss }
$$

$\begin{array}{llll}\text { a. } & \begin{array}{l}\text { al } \\ \text { bas } \\ \text { kii }\end{array} & \begin{array}{l}\text { al-ka } \\ \text { bas-ka } \\ \text { kii-ka }\end{array} & \begin{array}{l}\text { man } \\ \text { hair } \\ \text { stone }\end{array} \\ \text { b. } & \begin{array}{l}\text { sana } \\ \text { amak } \\ \text { sapaa }\end{array} & \begin{array}{l}\text { sana-ka } \\ \text { amak-ka } \\ \text { sapaa-ka }\end{array} & \begin{array}{l}\text { deer } \\ \text { bee } \\ \text { forehead }\end{array} \\ \text { c. } & \begin{array}{l}\text { suulu } \\ \text { kuhbil } \\ \text { baskarna }\end{array} & \begin{array}{l}\text { suu-ka-lu } \\ \text { kuh-ka-bil } \\ \text { bas-ka-karna }\end{array} & \begin{array}{l}\text { dog } \\ \text { knife } \\ \text { comb }\end{array} \\ \text { d. } & \begin{array}{l}\text { siwanak } \\ \text { anaalaaka } \\ \text { karasmak }\end{array} & \begin{array}{l}\text { siwa-ka-nak } \\ \text { anaa-ka-laaka } \\ \text { karas-ka-mak }\end{array} & \begin{array}{l}\text { root } \\ \text { chin } \\ \text { knee }\end{array}\end{array}$

There are two main virtues of this line of approach to morphology. First, it clearly avoids the duplication problem by getting rid of the lexical storage of affixes. This simplifies the theory. Second, the mixture of Item-and-Arrangement and Itemand-Process is avoided by deriving affixation from constraints.

But this alignment-based formalization of affixation is not valid. Considering the Ulwa examples, Russell's framework correctly accounts for the position of the 
possessive affix given Align $\left([\mathrm{ka}]_{\mathrm{Af}}, \mathrm{L}, \mathrm{Ft} t^{\prime}, \mathrm{R}\right)$, as shown in (18), where the brackets in (18c) represent the head foot.

\begin{tabular}{|ll||c|}
\hline & /siwanak/ & Align $\left([\mathrm{ka}]_{\mathrm{Af}}, \mathrm{L}, \mathrm{Ft}{ }^{\prime}, \mathrm{R}\right)$ \\
\hline \hline a. & ka-si.wa.nak & $* !$ \\
\hline b. & si-ka-wa.nak & $* !$ \\
\hline c. & (si.wa)-ka-nak & \\
\hline d. & si.wa.nak-ka & $* !$ \\
\hline
\end{tabular}

A crucial candidate missing here is [si.wa.nak] which does not contain the affix $k a$. Under the standard assumption that alignment constraints are vacuously satisfied when the variables specified in them do not exist in the candidate's phonological representation (e.g., Align(Ft,L,PrWd,L) is vacuously satisfied by $[\sigma \sigma \sigma \sigma]_{\mathrm{PrWd}}$ which contains no foot structure), [si.wa.nak] should be more harmonic than [si.wa.-ka-.nak] since the latter actual form incurs two Dep violations. Max plays no role here since the affix $k a$ does not exist in the underlying representation under the full pursuit of Item-and-Process proposed by Russell, as noted above. It appears to be tacitly assumed that the variables in the alignment constraint must show up in the candidates: it is essential that the grammar yield only candidates which contain the variables stated in the alignment constraint. But how is this guaranteed? Given Freedom of Analysis (Prince and Smolensky 1993; McCarthy and Prince 1993b:20), which maintains that any candidates can be permitted to be generated by Gen, nothing prevents the grammar from producing candidates without the variables in their representations. To ensure that the variables appear on the surface, some kind of Max must be included. Given the absence of the affixal element in the input, the 
input-output Max constraint is clearly irrelevant. Rather, the Max constraint needs to refer to the variables specified in the alignment constraint: the Max constraint needed here refers to $[\mathrm{ka}]_{\mathrm{Af} .}$ in the alignment constraint. But this constraint is peculiar since it requires mapping between part of a constraint and outputs. Such correspondence is unmotivated, so this move comes at great cost. This shows that the alignment-based approach to affixation is problematic too. Alternatively, for Max-IO to function properly, the affix $k a$ must be already present in the underlying representation, but this goes back to the duplication problem which Russell argues against. Essentially, this move is precisely the same as my position taken here, namely classifying morphemes into two groups: those with underlying phonological substance and those without it. Given that the complete abolishment of affixes as entities comes at great expense, I keep the traditional position that affixation (except reduplication) derives from fixed segmentism in the underlying representation.

In summary, I have examined the nature of RM and discussed how it should be conceptualized and formalized in order to achieve a comprehensive understanding of concatenative and nonconcatenative morphology. Given that making explicit various morphosyntactic functions is the primary role of morphology and that morphemes are primitive morphological units to substantiate this role, I argued that $\mathrm{RM}$ is the constraint which demands some overt phonological manifestation of every morpheme contained in the underlying representation. In other words, RM is the constraint which is responsible for maintaining morphological contrasts. RM merely requires morphemes to receive some surface phonological manifestation but does not specify what it should be. The formal mechanism to obtain a specific phonological exponent for morpheme realization is the topic to be addressed in the next section. 
Given the richness of the base principle as a fundamental property of OT, RM needs to compare output forms. I proposed a model in which candidates competing for the winner are compared against the output of the bare stem which carries no morphosyntactic function. It is crucial that the output form of the bare stem be at least a possible form rather than an actual independent word of the language. This does not preclude the possibility that a certain morphosyntactic category is derived from another morphosyntactic category although this is restricted to cases where there is some phonological evidence for the output-output correspondence. In such a case, the output of the bare stem does not enter the picture. The proposed conceptualization of RM has a number of desirable theoretical consequences as well. Given the model outlined here, morphemes are regarded as primitive morphological entities which must exist in the underlying representation. Reviewing a recent proposal made by Russell $(1995,1999)$ which abandons Item-and-Arrangement entirely, I argued that this alternative comes at great cost.

\subsection{Emergence of Nonconcatenative Morphology}

Building on what has been discussed, I propose a general schema of deriving various nonconcatenative morphological processes in this section. I discuss this section with the plural formation examples in German provided in (19). The examples are given orthographically for perspicuity.

$\begin{array}{lll}\text { Singular } & \text { Plural } & \text { Gloss } \\ & & \\ \text { Vater } & \text { Väter } & \text { father } \\ \text { Mutter } & \text { Mütter } & \text { mother } \\ \text { Bruder } & \text { Brüder } & \text { brother } \\ \text { Tochter } & \text { Töchter } & \text { daughter } \\ \text { Acker } & \text { Äcker } & \text { field }\end{array}$


There are three factors important to articulating a general schema of nonconcatenative morphology. First, nonconcatenative morphology involves faithfulness violations by modifying the phonological shape of the base (see section 2.2). Second, morphemes whose phonological manifestation depends upon a stem change have literally nothing as underlying phonological substance (see sections 2.2 and 2.3). Finally, RM plays a central role in the straightforward and integrated understanding of realizational morphology. These points are closely related to one another in the following way: some phonological exponent must appear for a morpheme contained in the underlying representation under duress of RM, but stem modification is the only eligible strategy when the morpheme contains no phonological material as its intrinsic property. In (19), for example, the plural morpheme does not possess any phonological substance, and therefore, the base stem must undergo some phonological reshaping as the signal of the plural morpheme. The specific strategy is umlauting a base vowel. This observation can be captured by ranking RM over Ident-IO-[+back], indicating that the identity constraint must be sacrificed for the satisfaction of RM. Generalizing this observation, faithfulness violations involved in nonconcatenative morphology are motivated by RM » Faith.

Now, an important question arises immediately: why does the base form (from which a form exhibiting nonconcatenative morphology is derived) not undergo any phonological changes triggered by RM? In other words, why is nonconcatenative morphology not so pervasive in a language, taking place anywhere regardless of morphosyntactic categories? Taking the plural formation in German, why do only plural forms (but not singular forms) undergo umlaut? This is a significant issue under my proposal that, without evidence for output-output correspondence, 
phonological substance of a stem is independent of any morphosyntactic categories. In the German data in (19), this assumption means that both singulars and plurals are derived from bare stems which are not associated with any morphosyntactic function. The singular and plural morphemes are attached to those bare stems (e.g., /Vater/) when singular (e.g., /Vater/Singular) and plural (e.g., /Vater/Plural) forms are in need. Recall from section 2.3 that RM compares two outputs. In the German case, the output of a bare stem (e.g., [Vater]) is the output to be compared against the output of $/$ Vater/ ${ }_{\text {Singular }}\left(\right.$ i.e., $\left.[\text { Vater }]_{\text {Singular }}\right)$ and that of $/$ Vater/ $/_{\text {Plural }}\left(\right.$ i.e., $\left.[\text { Väter }]_{\text {Plural }}\right)$. Given RM » Ident-IO-[+back], it is unexpected that the output of the singular form does not undergo umlaut. The question is why plural forms are subject to the umlaut process but singular forms are not.

My proposal is that a faithfulness constraint is subdivided into several indexed components. Relevant indexes are morphosyntactic categories. The dispersion of a faithfulness constraint is not unprecedented. Itô and Mester (1999b) develop the idea of stratum-specific faithfulness in their study of the Japanese lexicon (see also Itô and Mester 1995ab for their related work, and also Fukazawa, Kitahara and Ota 1998 for a similar line of argument). The core tenet is that a single faithfulness constraint is indexed with respect to lexical strata and those indexed faithfulness constraints are ranked differently with respect to markedness ones. The consequence is that distinct lexical strata exhibit different behaviors with respect to the satisfaction/violation of markedness constraints.

My proposal is an extension of this indexation idea to morphosyntactic categories (cf. Benua 1997:chapter 4). Suppose that a bare stem is given and that it serves as the phonological input of two morphosyntactic categories $\alpha$ and $\beta$ and that 
some stem modification occurs only in the output of $\beta$. Given this set-up, the distribution of nonconcatenative morphology can be captured by the ranking provided in (20). I call (20) the emergence of nonconcatenative morphology schema. In morphosyntactic category $\alpha$, preserving the phonological information of the bare stem is more valuable than satisfying RM. By contrast, satisfying RM is more important when the output of morphosyntactic category $\beta$ is evaluated. Another logical possibility would be $\mathrm{RM}_{\beta}$ » Faith » $\mathrm{RM}_{\alpha}$, relativizing $\mathrm{RM}$ instead of faithfulness constraints. I will discuss and reject this alternative in section 3.2.1.

The Emergence of Nonconcatenative Morphology Schema

Faith $_{\alpha} \gg$ RM » Faith $_{\beta}$

"Faith" in (20) is a variable, so a specific faithfulness constraint occupies its position. As encapsulated in (21), various nonconcatenative morphological processes are obtained by replacing the variable with specific faithfulness constraints.

\begin{tabular}{|c|c|}
\hline Morphological Process & Constraint Ranking \\
\hline Subtractive morphology & $\operatorname{Max}_{\alpha} » R M » \operatorname{Max}_{\beta}$ \\
\hline Umlaut, Suppletion, Mutation & Ident $_{\alpha} \gg \mathrm{RM} \gg$ Ident $_{\beta}$ \\
\hline Morphological epenthesis & $\operatorname{Dep}_{\alpha} \gg \mathrm{RM} \gg \operatorname{Dep}_{\beta}$ \\
\hline Metathesis & Linearity $_{\alpha} \gg \mathrm{RM} »$ Linearity $_{\beta}$ \\
\hline Infixation & Contiguity $_{\alpha} \gg \mathrm{RM} \gg$ Contiguity $_{\beta}$ \\
\hline Reduplication & Integrity $_{\alpha} » R M »$ Integrity $_{\beta}$ \\
\hline Haplology (Fusion) & Uniformity $_{\alpha} \gg \mathrm{RM} \gg$ Uniformity $_{\beta}$ \\
\hline
\end{tabular}

(22) and (23) illustrate how the emergence of nonconcatenative morphology schema works to explain the German examples in (19). In (19), the plural morpheme 
is phonologically realized through umlaut whereas the singular morpheme receives no overt phonological exponence. This suggests that the singular and the plural morphemes correspond to $\alpha$ and $\beta$ in (20) respectively. Moreover, umlaut causes the change of vowel frontness, so the relevant faithfulness constraint is Ident-IO-[+back]. In (22), Ident-IO-[+back $]_{\text {Singular }}$ outranks RM, and therefore, no nonconcatenative phonological change is permitted in the singular. RM is violated by the intended winner here because it is phonologically the same as the output of the bare stem (i.e., [Vater]). By contrast, in plural forms, a violation of Ident-IO-[+back $]_{\text {Plural }}$ is demanded since RM outranks this faithfulness constraint, as in (23). Given that the plural morpheme is contentless phonologically, violating Ident-IO-[+back $]_{\mathrm{Plural}}$ is the least costly strategy to substantiate the morpheme, assuming that all other faithfulness constraints bearing the plural morphosyntactic marking are ranked over Ident-IO$[+\mathrm{back}]_{\text {Plural }}$.

\begin{tabular}{|c|c|c|c|c|c|}
\hline & & /Vater/Singular & Ident-IO-[+back $]_{\mathrm{Sg}}$ & $\mathrm{RM}$ & Ident-IO-[+back $]_{\mathrm{Pl}}$ \\
\hline a. & $1 \rightarrow$ & Vater & & $*$ & N/A \\
\hline b. & & Väter & $* !$ & & N/A \\
\hline
\end{tabular}

\begin{tabular}{|c|c|c|c|c|}
\hline & /Vater/Plural & Ident-IO- $[+ \text { back }]_{\text {Sg. }}$ & $\mathrm{RM}$ & Ident-IO-[+back $]_{\mathrm{Pl} .}$ \\
\hline a. & Vater & N/A & $* !$ & \\
\hline & Väter & N/A & & $*$ \\
\hline
\end{tabular}

The German examples motivate relativization of a single faithfulness constraint with respect to various morphosyntactic categories. Given the system here, it is potentially possible that both Ident-IO- $[+ \text { back }]_{\text {Singular }}$ and Ident-IO- $[+ \text { back }]_{\text {Plural }}$ 
outrank RM in a given language when neither of the morphosyntactic categories carries inherent phonological content. Umlaut is expected both in the singular and the plural in this case, leading to ubiquitous front rounded vowels in the language. This is an unlikely situation, but given the markedness hierarchy to prefer unroundedness for front vowels, this situation is pretty unstable. It is likely that the language undergoes internal changes such that those front rounded vowels are unrounded over time by phonological adjustment by oncoming generations of language learners, in effect eliminating the predominant distribution of marked vowels.

The Aka examples presented earlier in $(7 \mathrm{a}, \mathrm{b})$ suggest another dimension of faithfulness relativization: a single morphosyntactic category is attached to various different faithfulness constraints. Focusing upon the voicing alternation of the singular forms, the change from [-voi] to [+voi] takes place, but not vice versa. To explain this uni-directionality, mono-dimensional Ident is not sufficient here. I assume featural Max and Dep constraints (Lombardi 1998) (see Pater 1999 for a proposal to decompose a single Ident constraint into two asymmetrical uni-directional subcomponents). As shown in (24) and (25), this relativization plays an important role to block phonological polarity. In (24) and (25), I assume that the class 5 singular morpheme does not contain any phonological material, but Akinlabi (1996) assumes that the featural prefix [voice] is contained as the phonological substance of the morpheme. Under the approach taken here, these two analyses do not make any different prediction as far as the data in $(7 a, b)$ are concerned. Given the system advocated here, $\mathrm{RM}$ is defined strictly phonologically, and therefore, (24a) violates $\mathrm{RM}$ regardless of whether [voice] is specified or not as the phonological material of the morpheme. 


\begin{tabular}{|c|c|c|c|c|}
\hline & /tegge/ Singular $_{\text {}}$ & Max-IO-[voi $]_{\mathrm{Sg}}$ & $\mathrm{RM}$ & Dep-IO-[voi $]_{\text {Sg. }}$ \\
\hline a. & tenge & & $* !$ & \\
\hline & denge & & & * \\
\hline
\end{tabular}

\begin{tabular}{|ll||c|c|c|}
\hline & /goala/ $_{\text {Singular }}$ & Max-IO-[voi $]_{\text {Sg. }}$ & RM & Dep-IO-[voi $]_{\text {Sg. }}$ \\
\hline \hline a. $\quad$ goala & & $*$ & \\
\hline b. & koala & $* !$ & & \\
\hline
\end{tabular}

The idea of relativized faithfulness is obscured in concatenative morphology, however. In English singular-plural pairs as in $\left[\mathrm{k}^{\mathrm{h}} æ \mathrm{t}\right]_{\text {Singular }} \approx\left[\mathrm{k}^{\mathrm{h}} æ \mathrm{ts}\right]_{\text {Plural }}$, there is not any phonological evidence to believe that plurals are derived from singulars, and therefore, they are both derived from bare stems. The fact that singular forms do not exhibit any phonological distinction from bare stems suggests that all singular faithfulness constraints are ranked over RM. This ranking ensures that singular forms sacrifice the satisfaction of RM because of high ranking faithfulness constraints, coupled with the assumption that the singular morpheme is phonologically contentless. The plural morpheme, by contrast, carries the affix /s/ as its inherent phonological substance, and therefore, faithful parsing of the affixal material suffices to satisfy RM. Crucially, parsing the underlying material is an issue of phonological faithfulness, and therefore, faithful parsing covers the role played by RM, suggesting that the ranking between RM and most singular-specific faithfulness constraints remains undetermined on empirical grounds.

But $\mathrm{RM}$ is not entirely inactive in concatenative morphology. In German, degemination generally takes place when morpheme concatenation creates a sequence of identical or similar segments (e.g., /tret-t/ $\rightarrow$ [trit] 'step', /halt-t/ $\rightarrow$ [helt] 'stop'). 
However, degemination is blocked under a certain circumstance and a schwa is epenthesized, as in $/$ rait-t/ $\rightarrow$ [rartat] 'ride, 3sg.' (Wiese 1996b; Klosa, ScholzeStubenrecht and Wermke 1998). The crucial observation is that degemination occurs when a stem undergoes some phonological change and therefore the relevant morphological information is denoted by it even if the affixal material does not surface. By contrast, schwa epenthesis enters the picture when no such stem change occurs since the affix is the only phonological element to express the relevant morphosyntactic information. More formally, the OCP motivates degemination, but the desire to express underlying morphemes overtly in the surface representation prevents the regular phonological process (Kawahara 2001). In a general vein, this situation is captured by RM (») markedness » faithfulness, as illustrated in (26). The existence of RM is strongly motivated here, and therefore, $\mathrm{RM}$ plays a crucial role not only in nonconcatenative morphology but also in concatenative morphology as well.

\begin{tabular}{|ll||l:l|l|}
\hline & $/$ rait-t $/_{\text {Plurral }}$ & RM & OCP & Dep \\
\hline \hline a. & rait & $* !$ & & \\
\hline b. & raitt & & $* !$ & \\
\hline c. & rartat & & & $*$ \\
\hline
\end{tabular}

RMT proposed here has an important theoretical repercussion. As shown in (21), truncatory morphology and reduplication are yielded at the expense of Max and Integrity violations respectively. Normally, TRUNC and RED have been assumed as morphemes requiring truncation and reduplication in the earlier literature (see Benua 1995, 1997 for truncation, and McCarthy and Prince 1993b, 1995, 1999; Urbanczyk 1998; Walker 2000 for reduplication). TRUNC and RED specify how they must be 
phonologically instantiated, but given RMT, such nonconcatenative morphemes are unnecessary. As a common property of concatenative and nonconcatenative morphology, I pointed out that they share the purpose of maintaining morphological contrasts. I claim that RM is the constraint which reflects this idea, but morphemes themselves do not have their own desire concerning their phonological exponence. Rather, it is determined by how the grammar ranks morpheme-specific faithfulness constraints with respect to other constraints such as RM and markedness constraints. Morphemes such as TRUNC and RED literally do not exist in the system of RMT. Truncation and reduplication surface when violating Max or Integrity is the cheapest faithfulness violations respectively (see later chapters for exemplification). The annihilation of such contentless but process-specific morphemes is empirically preferable because, if such morphemes existed, it is simply an unexplainable accident that the variation of such morphemes is pretty restricted (virtually, only truncation and reduplication). No language needs a morpheme like REVERSE-ONSET-ANDRIME which maintains that the onset and the rime must be reversed in every single syllable for the morpheme to be successfully incarnated phonologically. The only way to guarantee the absence of such unattested morphemes is to prohibit contentless but process-specific morphemes entirely.

This issue is related to the major argument against Item-and-Process. It is the weakness of the morphological model that available morphological processes are not constrained and therefore that Item-and-Process runs into an over-generation problem (Hockett 1954; Anderson 1992). The nature of the issue concerning TRUNC and RED is very similar to the lack of restrictiveness of Item-and-Process since there is no way to guarantee that TRUNC and RED are the only phonologically empty process- 
specific morphemes permitted in natural languages. The only way to avoid the unwanted over-generation problem is merely to abolish the existence of such morphemes in Universal Grammar (see Horwood 1999 for a similar argument).

Realizational morphology based upon the emergence of nonconcatenative morphology schema in (20) brings up another important theoretical implication: diacritics and floating features associated with morphemes lose their room. Umlaut in German, for example, was treated by Strauss (1976) such that a diacritic feature [+Umlaut] causes vowel fronting of a stem vowel. In RMT, all nonconcatenative morphology is accomplished by the emergence of nonconcatenative morphology schema, and therefore, at least a majority of diacritics and floating features can be eliminated from linguistic theory. Even cases where a secondary articulation (i.e., labialization, palatalization, velarization and glottalization) denotes the presence of a morphosyntactic category can be subsumed under the generalized schema since they would be successfully captured by positing appropriate Ident constraints. Although diacritics and floating features are convenient devices, any theory resorting to them cannot achieve a unified understanding of overall nonconcatenative morphology. The reason is that there is no reasonable way to establish a diacritic or floating feature that motivates subtractive morphology and metathesis. No new phonological material is added in these word formations, and therefore, any attempt to incorporate them under the rubric of diacritics or floating features would result in assuming an abstract morpheme similar to TRUNC and RED because the morpheme is phonologically contentless but process-specific. As discussed above, however, admitting such morphemes is empirically undesirable. RMT avoids this problem by reducing all nonconcatenative morphological processes to interactions of RM and phonological 
faithfulness constraints. This move conforms to the fundamental tenet of OT that sound alternations are surface outcomes of constraint interactions.

To close this section, let us sketch some more general properties of (20) in cases where some phonological constraint $\mathrm{C}$ is placed in various locations, anticipating the discussion in the next chapter. All the logical possibilities are given in (27). In (27a), $C$ is fully operative both in $\alpha$ and $\beta$, so $C$ must be always obeyed. By contrast, in (27d), C is overridden both by Faith ${ }_{\alpha}$ and by Faith $_{\beta}$, so $\mathrm{C}$ cannot compel any faithfulness violations. More interesting are cases illustrated in $(27 b, c)$. In these two cases, a phonological constraint $\mathrm{C}$ intervenes between the two faithfulness constraints. This is a typical instance of the emergence of the unmarked ranking schema (McCarthy and Prince 1994). In (27b), C crucially dominates RM so that the power of RM is potentially suppressed to fulfill the requirement imposed by C. In (27c), although $\mathrm{C} \gg$ Faith $_{\beta}$ still yields the emergence of the unmarked in $\beta$, nonconcatenative morphology is always observed because $\mathrm{C}$ cannot block the power of RM. I discussed that morphologically conditioned stem changes result in a more marked phonological representation. This generalization still holds since the stem change itself (e.g., umlaut) produces a phonologically less harmonic representation. Nevertheless, the output form is potentially better than the input phonologically in certain respects. But this does not mean that phonological changes on the stem are phonologically motivated in such cases. This issue will be taken up in section 3.2.1 in the context of subtractive morphology involved in Koasati plural formation.

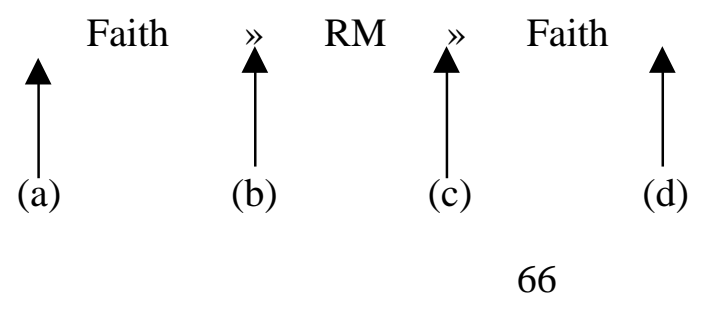




\subsection{Against Anti-Faithfulness Theory}

Given that nonconcatenative morphology exhibits anti-faithfulness effects, the immediate analytical possibility is to take advantage of anti-faithfulness theory advocated by Alderete (1999). In this section, I give an overview of his proposal and argue against the extension of the theory to the nonconcatenative morphological processes at issue here. Investigating the morphophonology of Yidin, Hayes (1997, 1999) independently suggests the possibility of anti-correspondence which actively requires morphemes to alternate in particular ways. But anti-correspondence is brought to the fore as an ad hoc (or crosslinguistically not well motivated) strategy to deal with alternations impossible to be handled by interactions of markedness and faithfulness constraints. Hayes $(1997,1999)$ thus regards anti-correspondence as a language-particular brute force acquisitional device rather than integral part of Universal Grammar. Alderete (1999) explicitly argues that anti-faithfulness theory must be incorporated as part of universal principles embedded in the OT system. For this reason, I review only Alderete (1999).

Alderete (1999) motivates and develops anti-faithfulness theory by pointing out empirical difficulties of the standard OT assumption that Con consists exclusively of markedness and faithfulness constraints. Phonological polarity (also known as $\alpha$ switching, flip-flop, or exchange) constitutes important empirical data in support of his proposal. In Luo, a Western Nilotic language spoken in Kenya and Tanzania, nominative singulars serve as the bases of their plural counterparts, as exemplified in (28) (Stafford 1967; Gregersen 1972; Okoth-Okombo 1982; Stonham 1994). The examples receive the following descriptive generalization: (i) a plural suffix $-i$ or $-e$ (phonetically realized either as $[\mathrm{e}]$ or as $[\varepsilon]$ ) is attached, (ii) a word-final vowel of a 
singular form is subject to deletion, and (iii) the voicing value of the final consonant is switched (i.e., [-voi] $\rightarrow$ [+voi], and vice versa). The earlier works regard the voicing alternation as a genuine instance of phonological polarity, leading to OkothOkombo's (1982) formulation of the plural formation in terms of the $\alpha$-notation convention (cf. Chomsky and Halle 1968).

\begin{tabular}{|c|c|c|}
\hline Singular & Plural & Gloss \\
\hline alot & alode & vegetable(s) \\
\hline bat & bade & $\operatorname{arm}(\mathrm{s})$ \\
\hline $\operatorname{lu} \theta$ & luð $\varepsilon$ & $\operatorname{stick}(s)$ \\
\hline $\operatorname{ruo\theta }$ & ruoठ̋i & chief(s) \\
\hline guok & guogi & $\operatorname{dog}(s)$ \\
\hline kidi & kite & stone(s) \\
\hline puoð̄o & puo@e & $\operatorname{garden}(\mathrm{s})$ \\
\hline got & gode & $\operatorname{twig}(\mathrm{s})$ \\
\hline t $\int 0 g o$ & t Joke & bone (s) \\
\hline $\mathrm{d} \varepsilon \mathrm{b} \varepsilon$ & dep $\varepsilon$ & debbi(s) \\
\hline
\end{tabular}

Building on the generalization made in the earlier literature, Alderete (1999) argues that polarity phenomena present a serious challenge to OT. Under the standard premise that Con consists only of markedness and faithfulness constraints, the output can never be phonologically more marked than the input, a property called harmonic ascent (Prince 1997; Moreton 1999; McCarthy 2000b). Consider a schematic polarity example: $/ \mathrm{A} / \rightarrow[\mathrm{B}]$ and $/ \mathrm{B} / \rightarrow[\mathrm{A}]$. Were $A$ phonologically more marked than $B, A$ could surface as $B$ if a markedness constraint militating against $A$ is ranked over a competing faithfulness constraint, but $B$ is never realized as $A$. The reverse holds when $A$ is less marked than $B$. This observation led Alderete (1999) to propose a new family of constraints: anti-faithfulness. For the case at hand, the relevant constraint is obtained through a negation of a faithfulness constraint IdentOO-[voi]. The negative operator attached to faithfulness constraints is existentially 
quantified such that $\neg$ Ident-OO-[voi] requires at least one pair of correspondent segments to be non-identical for the voicing feature. As demonstrated in (29) and (30), the voicing polarity in Luo is accounted for by giving privileged status to $\neg$ Ident-OO-[voi] over Ident-OO-[voi].

\begin{tabular}{|ll||c|c|}
\hline & /alot-e/ Plural & ᄀIdent-OO-[voi] & Ident-OO-[voi] \\
\hline \hline a. & alote & $* !$ & \\
\hline b. $\quad$ alode & & $*$ \\
\hline
\end{tabular}

\begin{tabular}{|ll||c|c|}
\hline & \multicolumn{1}{|c||}{$/$ kidi-e/ } & & \\
& Plural & Ident-OO-[voi] & Ident-OO-[voi] \\
\hline a. & kide & $* !$ & \\
\hline b. $\quad$ kite & & $*$ \\
\hline
\end{tabular}

Anti-faithfulness theory is subsequently applied to affix-controlled accent phenomena, where particular affixes affect the underlying accent of a stem in one way or another. Among others, Alderete (1999) discusses dominant affix effects in Japanese. As illustrated in (31), Japanese has three types of dominant affix phenomena: dominance effects, preaccentuation, and accent shift (McCawley 1968; Poser 1984b).

a. Dominance effect

Underlying /kóobe-kko/ /edo-kko/

b. Preaccentuation

Underlying /nisímura-ke/ /yosida-ke/
Output [koobe-kko] [edo-kko]

Output [nisimurá-ke] [yosidá-ke]
Gloss

native of Kobe native of Tokyo 
c. Accent shift

$\begin{array}{lll}\text { Underlying } & \text { Output } & \text { Gloss } \\ \text { /kúzu-ya/ } & \text { [kuzú-ya] } & \text { junkman } \\ \text { /toma-ya/ } & \text { [toma-ya] } & \text { mat seller }\end{array}$

First, the dominant affix $-k k o$ requires deletion of the base (underlying) accent, as in (31a). Thus, the entire word is accentless whether the base contains a lexical accent (as in [kóobe]) or not (as in [edo]). Second, (31b) shows examples of preaccentuation. The suffix -ke demands that the word accent be placed on the syllable immediately preceded by it. As a result, the base-final syllable carries accent both when the base has an original accent (as in [nisímura]) and when the base is accentless (as in [yosida]). Finally, (31c) illustrates accent shift. The crucial difference between (31b) and (31c) is that no accent insertion takes place in (31c) while it is obligatorily required in (31b). Abstracting away from various details, Alderete's proposal is that prosodic anti-faithfulness constraints are operative. More specifically, $\neg$ Max-OO-Prom, $\neg$ Dep-OO-Prom, and $\neg$ NoFlop-OO-Prom are relevant constraints ranked over their faithfulness counterparts in dominance effects, preaccentuation, and accent shift respectively. $\neg$ Max-OO-Prom actively requires deletion of an underlying accent, $\neg$ Dep-OO-Prom mandates for insertion of an accent not present underlyingly, and $\neg$ NoFlop-OO-Prom demands accent shift. Ranked over their faithfulness counterparts, the relevant accent modification is required, obtaining seemingly unmotivated various kinds of suprasegmental changes. Fundamentally the same basic analytical approach is taken to dominant affix effects in other languages.

Summing up, anti-faithfulness theory is intended to capture various phonological changes which take place on a stem for morphological reasons. In other words, there exists no principled phonological reason behind the voicing alternation in Luo and dominant affix effects in Japanese. They are morphologically conditioned 
instead. This suggests that anti-faithfulness theory is supposed to cover largely the same range of linguistic phenomena as those dealt with in this dissertation.

However, anti-faithfulness theory encounters several conceptual and empirical problems. First, admitting anti-faithfulness constraints significantly degrades the explanatory strength and restrictiveness of OT. An attractive tenet of OT is that various interactions of potentially conflictive constraints determine the ultimate output. Various constraints conflict with one another in different manners depending on the phonological configuration of the input. For example, consider a miniphonology where NoCoda outranks Max. Given the input /CVC/, the coda consonant is elided, but the input surfaces faithfully if the given input is /CV/. The two constraints are under tension only in a particular environment, but they are not incompatible with each other otherwise (i.e., context dependent). The point is thus that constraints themselves are not in conflict intrinsically. By contrast, antifaithfulness constraints are intrinsically in conflict with the faithfulness counterparts regardless of the context (i.e., context independent). Admitting anti-faithfulness constraints thus reduces the role played by faithfulness constraints and therefore undermines the explanatory power and the restrictiveness of OT. Rather, it is desirable to derive opposing effects without inherently antagonistic constraints.

By contrast, RMT based upon RM does not run into this problem. RM plays a key role in nonconcatenative morphology, but it is important in concatenative morphology as well. As discussed in section 2.4, RM is not incompatible with concatenative morphology where a morpheme contains some phonological material as its innate property. Importantly, RM and phonological faithfulness constraints are not ranked with respect to each other on empirical grounds in such cases. This 
indicates that RM and phonological faithfulness constraints are not antagonistic intrinsically.

Related to this point, another advantage of RMT is that the scope of its empirical coverage is wider than anti-faithfulness theory. While anti-faithfulness theory is intended to account specifically for anti-faithfulness effects as described above, RMT is intended to deal not only with stem modifications in nonconcatenative morphology but also with affixal morphology. This unification is not merely desirable from a conceptual point of view, but it turns out that the integrated understanding of the whole realizational morphology is a necessary move. As will be discussed in section 4.4, the actual aspect morpheme in Saanich contains a glottal stop as its phonological content, but it alternates with metathesis and reduplication. In cases where affixation and nonconcatenative stem changes coexist as allomorphs, it is problematic to separate concatenative morphology from nonconcatenative one. Rather, an integrated system should be constructed. RM simply requires every morpheme to receive some surface phonological manifestation, so it is not a constraint special to nonconcatenative morphology.

Another serious conceptual difficulty of anti-faithfulness theory is that it cannot explain the fact that anti-faithfulness effects are always morphologically governed. As aforementioned, the reason why nonconcatenative morphology incurs faithfulness violations is to express the existence of a morpheme on the surface. This cannot be directly captured by the anti-faithfulness approach. Anti-faithfulness constraints simply demand the output to be unfaithful to the input in a certain dimension. This means that they are not sensitive to the source of the driving force which causes faithfulness violations. More concretely, anti-faithfulness constraints 
are expected to be active whether the source is phonological or morphological. Antifaithfulness effects are restricted to cases where some morphological factor enters the picture (Anderson and Browne 1973; Moreton 1999), and therefore, nonconcatenative morphological processes such as subtractive morphology are never expected for purely phonological reasons. Alderete (1999) thus stipulates that anti-faithfulness constraints are active only in the surface-to-surface (or output-to-output) dimension to circumvent unwanted phonological exchanges, where an output is defined as a form which can stand on its own as an independent word in the sense of Benua (1997). Desirably, the system behind this stipulation should be formally understood.

By contrast, RM is sensitive to the existence of morphemes in the underlying representation. RM plays a role only when their phonological realization is at issue. It has nothing to say if no underlying morpheme exists which has not yet received any phonological manifestation. Most importantly, it follows that we do not need to restrict the domain where RM plays a role, avoiding the empirical problem stemming from the surface-to-surface restriction posed on anti-faithfulness constraints.

In addition to the conceptual problems, anti-faithfulness theory encounters an empirical drawback too. Crucial here is the nominative formation in Lardil in (32) (Hale 1973; Itô 1986; Wilkinson 1988; Weeda 1992; Prince and Smolensky 1993; Blevins 1997; Horwood 1999; Kurisu 1999).

$\begin{array}{lll}\text { Stem } & \text { Nominative } & \text { Gloss } \\ & & \\ \text { yalulu } & \text { yalul } & \text { flame } \\ \text { yiliyili } & \text { yiliyil } & \text { oyster } \\ \text { mayara } & \text { mayar } & \text { rainbow } \\ \text { kamputa } & \text { kamput } & \text { pandoanas nuts }\end{array}$

The crucial fact is that the stem forms cannot stand on their own as independent words, so they must be subtracted as in nominatives or otherwise 
inflected as in accusatives (-in). This fact is quite damaging to anti-faithfulness theory which maintains as a central claim that anti-faithfulness constraints operate only in the surface-to-surface (but not in the lexical-to-surface) dimension. Two responses are conceivable at this point. A first possibility is to exclude subtractive morphology from the realm of data that anti-faithfulness theory covers. This possibility is obviously undesirable since it means to give up explaining what it was arguably designed to account for. Indeed, anti-faithfulness theory is directly imported into an analysis of subtractive morphology by Horwood (1999) and Bat-El (2000). A second possible reaction is to eliminate the central stipulation that anti-faithfulness constraints participate only in the surface-to-surface correspondence. The reduced restrictiveness is a priori undesirable, but the relaxation of the key restriction gives rise to new empirical problems, especially in light of phonological polarity. As pointed out above, phonological polarity is stringently restricted to cases where some morphological condition comes into play, and this is indeed true in Luo, as exemplified in (28). Lexical-to-surface anti-faithfulness constraints expect the presence of purely phonological polarity effects, contrary to fact. This shows that neither response is satisfactory, and therefore, the nominative formation in Lardil constitutes quite strong empirical evidence against anti-faithfulness theory. Horwood (1999) applies anti-faithfulness theory to the nominative formation in Lardil, but this point is not discussed.

As articulated in section 2.3, RM is insensitive to the status of the input: it may or may not be an independent output form. This means that cases like Lardil, where base forms do not stand as full-fledged outputs, do not present any empirical problem to RMT. As discussed in 2.3, a bare stem serves as the input when a given 
morphosyntactic category is not derived from another category, and output candidates produced by Gen are compared against the output form of the bare stem input computed by the phonology of the language for the purpose of evaluating RM violations (see (8)). On the other hand, if the input is already an output in the language, candidates are directly compared with the input. The satisfaction/violation of RM can be computed regardless of the output status of the input (see section 3.2.2 for my analysis of Lardil nominalization).

Furthermore, RMT and anti-faithfulness theory are significantly different in terms of generality/specificity of RM and anti-faithfulness constraints. RM is a very general constraint in the sense that it does not specify how a given morpheme must be phonologically realized in the surface representation. By contrast, anti-faithfulness constraints are much more specific because they specify in what dimension stems undergo modification. For instance, $\neg$ Max requires that the stem change be in the form of deletion, and therefore, any other stem changes are regarded unsatisfactory. RM accepts any particular phonological exponence. This difference gives rise to a fundamental question: are morphemes process-specific in the sense that a particular process is required for the satisfaction of their phonological realization? Given a number of cases where a single morpheme is associated with multiple allomorphs (especially cases where allomorphs are nonconcatenative to be discussed in chapter 4), it is not plausible that morphemes intrinsically specify their phonological instantiation. Rather, a more promising idea would be to consider morphemes as entities whose desire is merely to receive some phonological exponence whatever it is, although phonological substance of affixes must be encoded lexically since it is an unpredictable innate property. 
Summarizing the argument in this section, I discussed that anti-faithfulness theory is faced with several conceptual and empirical problems. From the conceptual point of view, it brings analytical redundancy in the theory of OT, and as a result, it reduces the importance of faithfulness constraints and the explanatory power of OT. Moreover, anti-faithfulness constraints fail to capture the fact that faithfulness violations are forced to denote the presence of a morpheme overtly in the surface representation, resulting in a stipulation that anti-faithfulness constraints play an active role only in the surface-to-surface domain. On the empirical side, I argued that the nominative formation in Lardil cannot be handled by anti-faithfulness theory since base forms are not independent output forms. Any attempt to exclude or include the data either results in the loss of explanatory strengths or creates new empirical problems. Given these difficulties, no superficial fix would ameliorate the situation since these problems come directly from the fundamental set-up of antifaithfulness theory.

\subsection{Summary}

In this chapter, I investigated the formal characteristics of stem modifications involved in nonconcatenative morphology and outlined the theoretical mechanism to derive them. In the course of discussion, concatenative and nonconcatenative morphology were compared toward a unified understanding of realizational morphology. The most striking property of nonconcatenative morphology which differentiates it from concatenative morphology is anti-faithfulness effects. Antifaithfulness effects must be distinguished from faithfulness violations in regular

phonology because the former are morphologically governed. Theoretically 
interesting in the context of OT is the fact that faithfulness violations in nonconcatenative morphology do not improve phonological harmony. Rather, unlike in phonology, they yield a phonologically more marked representation. This is of great significance since nonconcatenative stem changes cannot be explained by interactions of faithfulness and markedness constraints, and therefore, they present an empirical challenge to OT.

I proposed in section 2.3 that RM plays a central role in an integrated understanding of concatenative and nonconcatenative morphology. RM requires all morphemes contained in the underlying representation to receive some phonological exponence. The specific exponent differs from morpheme to morpheme and also from context to context. But RM does not demand a particular phonological realization of a morpheme. Among others, this is a crucial difference between RM and anti-faithfulness constraints. As an evaluation metric of RM, I argued that output-output comparisons need to be established. This is not only conceptually required in OT but also desirable on an empirical basis. Morphosyntactic categories are derived from bare stems when no independent phonological evidence is there for output-output correspondence. In such cases, RM compares output candidates and the output form of the bare stem which is computed by the phonology (i.e., constraint ranking) of a given language. By contrast, when some independent evidence shows the need of output-output correspondence, a morphosyntactic category is derived from another one, and RM directly compares the input and the output candidates. An important underlying assumption in RMT is that morphemes are entities which exist as part of the underlying representation. Assessing an alternative position taking full Item-and-Process OT morphology, I argued that this possibility comes at great cost. 
Taking advantage of RM, I developed a general schema in section 2.4 to explain various nonconcatenative morphological operations in a unified and principled manner. They occur because a phonological faithfulness constraint ranked below RM must be sacrificed when a morpheme does not contain any phonological material. Faithfulness constraints are relativized with respect to morphosyntactic categories. By ranking them differently with respect to RM, the presence or absence of stem modification is determined for a given morpheme.

Finally, in section 2.5 , I reviewed anti-faithfulness theory articulated by Alderete (1999). It is not a desirable mechanism for nonconcatenative morphological phenomena given an empirical problem presented by Lardil subtractive morphology. I also argued that the theory is vulnerable to many general problems on conceptual grounds. Especially, the stipulation that anti-faithfulness constraints are active only in the output-output dimension and the inability to account for Lardil nominalization are closely interconnected. There would be no principled solution to circumvent both problems. Although anti-faithfulness theory would be an immediate and direct analytical possibility to analyze the range of nonconcatenative morphology phenomena given anti-faithfulness effects exhibited by them, I argued that it is not a viable idea. 


\section{Chapter 3}

\section{Truncatory Morphology}

\subsection{Introduction}

This chapter is devoted to exemplification of the general proposal made in chapter 2 . Empirical data investigated in this chapter all come from morphological truncation. Two types of morphological truncation are observed in natural languages: subtractive morphology and templatic truncation. In the earlier literature, templatic truncation has attracted a considerable amount of attention, but subtractive morphology has been studied much less intensively. They are similar in that part of the base is subject to deletion, but they exhibit important formal differences as well. In this chapter, I examine both of them, although subtractive morphology is more central to the principal topic of this dissertation. Nevertheless, I claim that subtractive morphology and templatic truncation are still governed by the same underlying principles and that they are to be accounted for in a unified way.

A first remarkable difference between subtractive morphology and templatic truncation is that the former is associated with the creation of some morphosyntactic category whereas the latter is not. In other words, subtractive morphology is grammaticalized in the language, but templatic truncation is not. Templatic truncation is employed most typically for hypocoristic forms, but they are rather outside the domain with which formal linguistics is concerned although hypocoristics would have some connotation in a social context. One might claim that templatic truncation is not part of language competence, but this is not true. As cogently argued by Bagemihl (1989), Itô, Kitagawa and Mester (1996), and Sanders (1999) 
among others in the context of language games, non-grammaticalized morphological operations are still tightly regulated by the general morphological and phonological principles operative in grammaticalized processes, and indeed, this holds of templatic truncation. I argue that a parallel explanation can be given for subtractive morphology and templatic truncation within the theoretical machinery developed in chapter 2 .

Second and more importantly, the residue of deletion is prosodically constant in templatic truncation (e.g., hypocoristic forms are basically of bimoraic size in Japanese, as in [Kazu-tyaN] from [Kazuhiko] where -tyaN is a hypocoristics-specific suffix), but this generalization does not hold of subtractive morphology. On the contrary, what is constant is what is deleted. Put differently, the deleted material can be phonologically defined, and the phonological size of the input does not have any influence on the eventual output in templatic truncation (i.e., the larger the input is, the more is deleted) whereas the size of the output is affected by that of the input in subtractive morphology. The two types of deletion are thus mirror-images. This is an important point especially for a framework like OT which evaluates only the wellformedness of output representations. An interesting puzzle is how mirror-imaged subtractive morphology and templatic truncation are successfully explained in an integrated fashion. Investigating templatic truncation is instructive in highlighting formal aspects of subtractive morphology as well.

The remaining portion of this chapter is organized as follows. In section 3.2, I discuss subtractive morphology as a representative phenomenon of nonconcatenative morphology. Despite the fact that it has been discussed less than templatic morphology, various analytical proposals have been made toward a satisfactory 
formal explanation within both a derivational framework and OT. The languages investigated are Koasati, Lardil, Icelandic and Hessian German. I demonstrate that all of these cases are accounted for in a principled way with the theoretical architecture advanced in chapter 2. In the course of discussion, various earlier derivational and constraint-based attempts are reviewed, and I argue for analyses framed in RMT. Section 3.3 is allotted to a case study of templatic truncation. Concrete examples will be drawn from loanword clipping in Japanese. Although deriving a templatic effect is an interesting issue on its own, as can be seen from a recent growing body of a-templatic analyses of reduplication (cf. Spaelti 1997; McCarthy and Prince 1999 as well as many other works) and templatic effects in Semitic morphology (Ussishkin 2000), our focus will be on how templatic truncation can be understood in parallel with subtractive morphology in spite of the formal differences stated above. Finally, section 3.4 is a summary of the main results.

\subsection{Subtractive Morphology}

Subtractive morphology is the opposite of overt affixation in that part of a base undergoes elision to derive a certain morphosyntactic category. Before beginning to discuss concrete data, it is beneficial to provide the typology of subtractive morphology. In many subtractive morphology examples, one or more concomitant regular phonological processes are involved, and therefore, subtractive morphology is often obscured. Setting those phonological complications aside, three patterns are observed crosslinguistically: final segment deletion, final syllable deletion, and final rime deletion. Although Weeda (1992) documents a few examples of word-initial subtraction, such examples are scarce and would have marginal status at best. 
Examples of final segment deletion include Danish imperatives (Anderson 1975; Jones and Gade 1981; Allan, Holmes and Lundskær-Nielsen 1995), the Rotuman incomplete phase (Churchward 1940; Haudricourt 1958ab; Biggs 1959, 1965; Milner 1971; Cairns 1976; Saito 1981; van der Hulst 1983; Janda 1984; McCarthy 1986b, 1989, 2000c; Mester 1986; Besnier 1987; Hoeksema and Janda 1988; Odden 1988; Anttila 1989; Weeda 1992; Blevins 1994), Icelandic deverbal nouns (Orešnik 1972, 1978ab; Arnason 1980; Kiparsky 1984; Itô 1986; Benua 1995), Lardil nominatives (Hale 1973; Itô 1986; Wilkinson 1988; Weeda 1992; Prince and Smolensky 1993; Blevins 1997; Horwood 1999; Kurisu 1999), Tiberian Hebrew imperatives (Prince 1975; Benua 1995, 1997), and Tiberian Hebrew jussives (Prince 1975; McCarthy 1979; Benua 1995, 1997). Some examples of languages with final syllable deletion are Papago perfectives (Hale 1965; Pranka 1983; Zepeda 1983; Lombardi and McCarthy 1991; Hill and Zepeda 1992; Weeda 1992; Golston 1996; Fitzgerald 1997; Horwood 1999), Alabama plurals (Broadwell 1993), and Koasati plurals (Martin 1988; Kimball 1983, 1991; Weeda 1992; Horwood 1999). Finally, those languages with final rime deletion are represented by Koasati plurals (Martin 1988; Kimball 1983, 1991; Lombardi and McCarthy 1991; Weeda 1992; Kurisu 1999), and plurals in Alabama, Choctaw, Chicksaw, and Mikasuki (Broadwell 1993).

Final segment deletion is the morphological subtraction with minimal divergence from the base. By contrast, final syllable elision is the case of maximal subtraction to the best of my knowledge. If this observation is true, the range of variations seen in subtractive morphology is surprisingly narrow. A prediction arising from this restriction is that there exist languages where a subsyllabic constituent larger than a single segment undergoes clipping. This is indeed confirmed 
by the cases of rime deletion enumerated above. Now, an interesting question is why languages do not employ any deletion larger than a monosyllable. The answer is presumably that foot subtraction, for example, cannot apply to minimal words given that the residue must not be phonologically null. If this did occur, it would present a serious challenge to language learners because they must detect rich morphosyntactic information from null phonology (i.e., non-recoverability). Related to the argument against zero morphs in section 2.3 , it is highly unlikely that phonological zero plays a significant role in realizational morphology. Given the theory of prosodic morphology where the prosodic hierarchy and foot binarity (at the moraic or syllabic level) are assumed, a minimal word can be defined either as a bimoraic foot or as a disyllabic foot (McCarthy and Prince 1986 et seq.). Were foot subtraction productively exploited to express various morphosyntactic functions, the base has to be at least trisyllabic to circumvent null strings, but no such minimality condition has been reported in the literature.

Below, I discuss how the proposal made in chapter 2 is implemented to explain real language data. I discuss the following four languages: Koasati (section 3.2.1), Lardil (section 3.2.2), Icelandic (section 3.2.3), and Hessian German (section 3.2.4). In the course of discussion, I provide critical assessment of various earlier approaches to subtractive morphology. Section 3.2.5 is a summary of this section.

\subsubsection{Koasati}

Koasati, a Muskogean language spoken in Lousiana, presents subsyllabic (rime) deletion in the plural formation (Martin 1988:230-231; Kimball 1983, 1991:314-334; Lombardi and McCarthy 1991:62-63; Weeda 1992; Horwood 1999; Kurisu 1999). 
Given that many lexical items express pluralization not through subtraction, those which undergo the process are lexically specified, but rime subtraction is regularly found in many lexical items. Examples are given in (1), where regular penultimate word stress is omitted. I call rime subtraction class I pluralization for the reason to be clear later. Since various segmental changes in the examples take us far afield, they are not discussed below. Rather, I focus on the subtractive effect on roots. Each of the forms below consists of a verbal root followed by one of the auxiliary suffixes and a discourse function suffix $-n$. The choice of a particular auxiliary suffix form is quite arbitrary: at least, there is no coherent phonological factor here. Some examples show that the auxiliary suffix is the same between the singular and the plural forms (e.g., $[\text { misip-li-n }]_{\text {Singular }} \approx[\text { mis-li-n }]_{\text {Plural }}$ ). This shows that the variation of the auxiliary suffix does not have any correlation with the plural formation.

\section{Class I pluralization}

$\begin{array}{lll}\text { Singular } & \text { Plural } & \text { Gloss } \\ \text { pitaf-fi-n } & \text { pit-li-n } & \text { slice up the middle } \\ \text { socaf-fi-n } & \text { sos-li-n } & \text { strip skin off something } \\ \text { tiwap-li-n } & \text { tiw-wi-n } & \text { open something } \\ \text { wilap-liici-n } & \text { wil-liici-n } & \text { tear up the earth } \\ \text { ciłip-ka-n } & \text { cif-ka-n } & \text { spear something } \\ \text { misip-li-n } & \text { mis-li-n } & \text { wink } \\ \text { koyof-fi-n } & \text { koy-li-n } & \text { cut something } \\ \text { simat-li-n } & \text { sim-mi-n } & \text { cut up tanned skin } \\ \text { iyyakohop-ka-n } & \text { iyyakof-ka-n } & \text { trip } \\ \text { fotop-ka-n } & \text { fot-ka-n } & \text { pull up something } \\ \text { obakhitip-li-n } & \text { obakhit-li-n } & \text { go backwards } \\ \text { akocofot-li-n } & \text { akocof-fi-n } & \text { jump down } \\ \text { facoo-ka-n } & \text { fas-ka-n } & \text { flake off } \\ \text { apołoo-ka-n } & \text { apot-ka-n } & \text { sleep with someone } \\ \text { atakaa-li-n } & \text { atak-li-n } & \text { hang something } \\ \text { icoktakaa-li-n } & \text { icoktak-li-n } & \text { open one's mouth } \\ \text { acokcanaa-ka-n } & \text { acokcan-ka-n } & \text { quarrel with someone } \\ \text { albitii-li-n } & \text { albit-li-n } & \text { place on top of } \\ \text { atinii-li-n } & \text { atin-ni-n } & \text { burn something } \\ \text { acitii-li-n } & \text { acit-li-n } & \text { tie something }\end{array}$


Given the emergence of nonconcatenative morphology schema proposed in section 2.4 (i.e., Faith ${ }_{\alpha} »$ RM » Faith ${ }_{\beta}$ ), subtractive morphology involved in the plural formation is captured by $\mathrm{RM} \gg \operatorname{Max}_{\mathrm{Plura}(\mathrm{I})}$. This is not sufficient, however. In (1a), where root-final $\mathrm{VC}$ is subject to deletion, $\mathrm{RM} » \mathrm{Max}_{\text {Plural }}$ ensures root-final consonant elision, assuming that some independent mechanism protects suffixal elements as well as the root-initial portion. What then motivates further vowel deletion? Here I adopt Align-R(Root,C) which requires roots to be closed by a consonant. Since roots undergo deletion, the final consonant does not have to be the one in the underlying representation. This is analogous to Final-C which demands that words be closed by a consonant (McCarthy 1993), expressed as Align-R(PrWd,C). As illustrated in (2), $\mathrm{RM}$, Align-R(Root,C) » $\mathrm{Max}_{\text {Plural(I) }}$ captures rime subtraction in (1a). RM » $\operatorname{Max}_{\text {Plural(I) }}$ motivates consonant deletion, and additionally, further vowel deletion is motivated by Align-R(Root,C). Essentially the same analysis holds of (1b).

\begin{tabular}{|ll||c:c|c|}
\hline & /pitaf-li-n/Plural(I) & RM & Align-R(Root,C) & $\operatorname{Max}_{\text {Plural(I) }}$ \\
\hline \hline a. & pi.taf.lin & $* !$ & & \\
\hline b. & pi.ta.lin & & $* !$ & $*$ \\
\hline c. $\quad$ pit.lin & & & $* *$ \\
\hline
\end{tabular}

Three remarks are in order here. First, rime subtraction in Koasati is a case of the emergence of the unmarked. A markedness constraint is ranked over $\operatorname{Max}_{\text {Plural(I) }}$, so plural forms must obey the phonological requirement imposed by the markedness constraint. This is seen from the fact that singular roots may end either with a vowel or with a consonant, but roots in the plural are consistently closed by a consonant. Given Align-R(Root,C), one might claim that the examples in (1b) can be explained 
without RM, but this is not true. The roots of the bases in (1a) are closed by a consonant, and therefore, no deletion is expected in those cases if rime deletion is not morphological. As the fatal violation of RM incurred by (2a) shows, rime subtraction is morphologically conditioned. Thus, effects of emergence of the unmarked in nonconcatenative morphology are a secondary factor parasitic upon morpheme realization.

A second point has to do with grammar dependency. Alderete (1999) and Horwood (1999) argue that anti-faithfulness theory is grammar dependent in the sense that interactions of constraints other than anti-faithfulness ones play an essential role in the determination of the final outcome. Grammar dependency is not special to anti-faithfulness theory, however. Under the RMT analysis here, another constraint Align-R(Root,C) is ranked over $\operatorname{Max}_{\text {Plural }}$. It is not $\mathrm{RM} » \operatorname{Max}_{\text {Plural(I) }}$ alone that is required to generate the correct output form. RMT is in a sense a more interactive system than anti-faithfulness theory. Anti-faithfulness constraints not merely require non-identity between two forms but also specify how their phonological non-identity must be implemented. By contrast, RM is simply fulfilled as long as some nonidentity is observed. The specific phonological instantiation depends upon interactions of RM with faithfulness constraints. Which faithfulness constraint is ranked beneath RM determines the specific stem modification.

Third, the fact that singular forms do not undergo morphological subtraction and that singular roots do not have to be closed by a consonant as in (1b) suggests

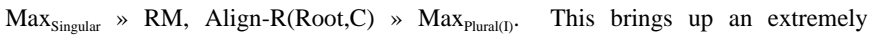
important question concerning the way that RM is evaluated. I proposed in section 2.3 that each candidate is compared with the output of the relevant bare stem. The 
latter is a form which is phonologically the optimal with respect to the phonology of the language. Given the idea that a faithfulness constraint can be subdivided into independent constraints carrying different morphosyntactic functions, how is the output of a bare stem determined when a phonological constraint intervenes between those faithfulness constraints? In Koasati, Align-R(Root,C) is sandwiched between $\operatorname{Max}_{\text {Singular }}$ and $\mathrm{Max}_{\text {Plural(I) }}$, and therefore, the optimal output of a bare stem is influenced by the relative ranking between Align-R(Root,C) and Max: Max » Align-R(Root,C) yields faithful outputs, but Align-R(Root,C) » Max generates unfaithful stem outputs in (1b). This difference is crucial since it affects the computation of RM violations. This problem does not arise in cases where the phonological constraint at issue is always satisfied or violated. For instance, word-final devoicing (WFD) in German takes place across the board, meaning that WFD » Ident-IO-[voi] holds of all morphosyntactic categories (see section 2.3).

My answer to this question is that the ranking granting higher faithfulness is chosen (i.e., Max » Align-R(Root,C) in Koasati). The important point is that the output of a bare stem is affected only by the phonology of the language. The fact that $\operatorname{Max}_{\text {Singular }}$ and $\operatorname{Max}_{\text {Plual(I) }}$ are separated by some phonological markedness constraint suggests that segmental deletion is morphologically conditioned. Since the bare stem does not carry any morphosyntactic category, it should not undergo any influence of morphologically governed phonological operations. The influence of morphological conditions can be avoided by ranking faithfulness constraints over markedness constraints.

In addition to rime deletion, a number of plural forms are obtained through final coda deletion (to be called class II pluralization), as exemplified in (3). The 
examples are excerpted from Martin (1988:232). The plural forms with coda deletion lengthen the root-final vowel. This is considered as a compensatory lengthening effect, which can be explained by high ranked Ident-IO- $\mu$ (Katayama 1998:61). This faithfulness constraint is irrelevant in rime deletion since the output does not contain the weight bearing elements of the underlying representation.

\section{Class II pluralization}

$\begin{array}{lll}\text { Singular } & \text { Plural } & \text { Gloss } \\ \text { famot-ka-n } & \text { famoo-ka-n } & \text { wave } \\ \text { libat-li-n } & \text { libaa-li-n } & \text { get burned by a hot solid } \\ \begin{array}{l}\text { asipat-li-n } \\ \text { tabat-ka-n }\end{array} & \text { tabaa-ka-n } & \text { get a splinter } \\ \text { topat-ka-n } & \text { topaa-ka-n } & \text { recede } \\ \text { akapos-ka-n } & \text { akapoo-ka-n } & \text { be pinched } \\ \text { labos-li-n } & \text { laboo-li-n } & \text { extinguish something } \\ \text { alabos-li-n } & \text { alaboo-li-n } & \text { close up (of flowers) } \\ \text { fibos-li-n } & \text { fiboo-li-n } & \text { squash something } \\ \text { fatof-ka-n } & \text { fatoo-ka-n } & \text { melt } \\ \text { yicof-ka-n } & \text { yicoo-ka-n } & \text { shrivel } \\ \text { dicof-fi-n } & \text { ficoo-li-n } & \text { chip by accident } \\ \text { kocof-fi-n } & \text { kocoo-li-n } & \text { pinch something } \\ \text { asikop-li-n } & \text { asikoo-li-n } & \text { breathe } \\ \text { kaca4-4i-n } & \text { kacaa-li-n } & \text { bite something }\end{array}$

The comparison between (3) and (1a) shows that the selection of rime/coda deletion must be lexically determined because CVCVC roots, for example, sometimes undergo rime deletion but coda deletion occurs in other occasions. My explanation is that $\operatorname{Max}_{\text {Plural }}$ is indexed with respect to two classes: the class of rime deletion (class I) and that of coda deletion (class II) and that $\operatorname{Max}_{\text {Plural(II) }}$ is ranked between RM and Align-R(Root,C) while Max $_{\text {Plural(I) }}$ is ranked below Align-R(Root,C), as shown in (2). $\operatorname{Max}_{\text {Plural(II) }}$ » Align-R(Root,C) permits only minimal deletion, as tabulated in (4). This is because RM is satisfied by deletion of only one segment and the phonological markedness constraint is not important in class II plurals. 
(4)

\begin{tabular}{|ll||c|c|c|}
\hline & /famot-ka-n/Plural(II) & $\mathrm{RM}$ & $\operatorname{Max}_{\text {Plural(II) }}$ & Align-R(Root,C) \\
\hline \hline a. & fa.mot.kan & $* !$ & & \\
\hline b. $\quad$ fa.moo.kan & & $*$ & $*$ \\
\hline c. & fam.kan & & $* * !$ & \\
\hline
\end{tabular}

At this point, it is in order to compare this analysis with Horwood's (1999). His approach to subtractive morphology rests upon Alderete's (1999) anti-faithfulness theory. I have already provided an empirical argument against the application of the theory to subtractive morphology (see section 2.5), but it is still worth reviewing the concrete implementation. He proposes the subtractive morphology model depicted in (5) (Horwood 1999:5).

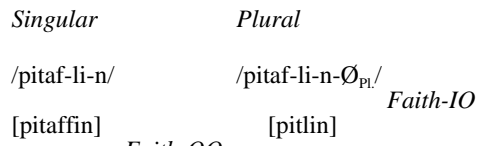

Faith-OO

The key constraint in his analysis is $\neg \mathrm{Max}-\mathrm{O}_{\mathrm{Sg} .} \mathrm{O}_{\mathrm{Pl}}-\mathrm{V}$, where the two outputs correspond to the singular and the plural. Abstracting away from the precise locus of segmental deletion, which is discussed by Horwood (1999:section 3), the last vowel within the root must undergo elision because of high ranking $\neg$ Max $-\mathrm{O}_{\mathrm{Sg}} \mathrm{O}_{\mathrm{Pl} .}-\mathrm{V}$. The reason why the root-final consonant is also deleted is that $*$ Complex dominates the faithfulness constraints which militate against segmental deletion (Max-IO as well as Max-OO which is not discussed by Horwood). As demonstrated in (6), *Complex and $\neg \mathrm{Max}-\mathrm{O}_{\mathrm{Sg}} \mathrm{O}_{\mathrm{Pl}}-\mathrm{V}$ force final segment deletion and the deletion of the vowel preceding it respectively. The gist of Horwood's approach is condensed in this brief summary. $\neg$ Max specific to some output-output dimension outranks competing 
faithfulness constraints. This obtains the effect of morphological subtraction. Any additional deletion is motivated by interactions of other universal constraints.

\begin{tabular}{|ll||c|c|c|}
\hline & /pitaf-li-n- $\emptyset_{\text {Plural }} /$ & $*$ Complex & $\neg$ Max- \\
\hline \hline ag. & $\mathrm{O}_{\mathrm{Pl} .}-\mathrm{V}$ & Max-IO \\
\hline b. & pi.taf.lin & & $* !$ & \\
\hline c. & pitf.lin & $* !$ & & $*$ \\
\hline
\end{tabular}

I pointed out in section 2.5 that anti-faithfulness theory encounters various general conceptual problems as well as an empirical difficulty in the context of subtractive morphology. They are not the only problems, however. Another problem concerns the restrictiveness of the theory. As a problem for anti-faithfulness theory, Horwood (1999:section 3.4) points out its lack of restrictiveness. There is potentially no upper limit to the number of anti-faithfulness constraints which can outrank competing faithfulness constraints. More concretely, under anti-faithfulness theory, nothing prevents the possibility that $\neg$ Max, $\neg$ Linearity, and $\neg$ Integrity outrank their faithfulness counterparts, producing deletion, metathesis, and reduplication to denote the presence of a single morpheme. No such language exists to the best of my knowledge. We could limit the number of $\neg$ Faith » Faith ranking to one, but as Horwood admits, this is nothing more than a stipulation which does not follow from any part of the architecture of anti-faithfulness theory. This is a serious empirical problem since anti-faithfulness theory predicts the existence of many more nonexistent cases than actually attested types of examples.

This overgeneration problem does not arise in RMT, the central idea being that nonconcatenative morphology such as subtractive morphology follows from RM 
» Faith ${ }_{\alpha}$ when morpheme $\alpha$ does not contain any phonological substance. For more than one nonconcatenative stem change to occur, it is a prerequisite that more than one Faith ${ }_{\alpha}$ is ranked below RM. Suppose that Max $_{\alpha}$ and Linearity ${ }_{\alpha}$ are dominated by RM. The question is whether there is any chance that subtractive morphology (violating $\mathrm{Max}_{\alpha}$ ) and metathesis (violating Linearity ${ }_{\alpha}$ ) cooccur for the purpose of substantiating morpheme $\alpha$. Given the doctrine of strict dominance of constraints, either $\operatorname{Max}_{\alpha}$ » Linearity or Linearity $_{\alpha}$ » $\operatorname{Max}_{\alpha}$ should hold in this scenario. As shown in (7), the candidate undergoing both subtraction and metathesis is harmonically bounded by the candidates which undergo either one of them in the sense of Prince and Smolensky (1993:176-178). In effect, the candidate violating the lower ranked faithfulness constraint is opted for as the winning form. Important here is that maximally one nonconcatenative stem change is permitted in RMT, eschewing the overgeneration problem. More than one morphological stem change is mechanically prevented. This issue will be taken up again in section 5.7.

\begin{tabular}{|c|c|c|c|c|c|}
\hline & & /ABCD/ & $\mathrm{RM}$ & $\operatorname{Max}_{\alpha}$ & Linearity $_{\alpha}$ \\
\hline a. & & $\mathrm{ABCD}$ & $* !$ & & \\
\hline b. & $1 \rightarrow$ & $\mathrm{ABC}$ & & $*$ & \\
\hline c. & 18 & ACBD & & & * \\
\hline d. & & $\mathrm{ACB}$ & & $*$ & * \\
\hline
\end{tabular}

Besides the difference of the fundamental conceptualization of subtractive morphology (i.e., RMT versus anti-faithfulness theory), my analysis departs from Horwood's (1999) in the treatment of the subtractive variations. As reviewed above, he analyzes the core of rime deletion as vowel elision, and the root-final consonant is 
subject to deletion for an independent phonological reason. Given $\neg \mathrm{Max}-\mathrm{O}_{\mathrm{Sg}} \mathrm{O}_{\mathrm{Pl}}-\mathrm{V}$ for the data in (1), another anti-faithfulness constraint must be postulated to account for the coda deletion cases in (3). He argues that $\neg \mathrm{Max}-\mathrm{O}_{\mathrm{Sg}} \mathrm{O}_{\mathrm{PI}}-\mathrm{Seg}$ is activated by the lexical items undergoing coda deletion. This analytical difference gives rise to a broader question of how lexical idiosyncrasies are best accounted for among various possibilities such as constraint reranking (Itô and Mester 1995b), lexicon-specific constraints (Pater 1995), and prespecification (Inkelas, Orgun and Zoll 1997; Inkelas 1999), but this does not pertain to us here.

What is important for our purposes is that the postulation of $\neg \mathrm{Max}-\mathrm{O}_{\mathrm{Sg}} \mathrm{O}_{\mathrm{Pl}}-\mathrm{V}$ makes the crosslinguistically incorrect prediction that there exist cases of some morphological constituent-internal subtractive morphology. Under Horwood's analysis, root-final consonant deletion is motivated for a syllable wellformedness reason, but suppose that there is a language in which such syllable wellformedness constraints are not important. Only one segment undergoes elision in this case. Given /CVCVC/ as the underlying base, then $\neg \mathrm{Max}-\mathrm{OO}-\mathrm{V}$ » Max-IO/OO produces [CVCC] as the optimal output. But as discussed by Horwood (1999) and in section 3.2 above, there exists no internal subtraction of a morphological constituent employed for morphological reasons. This argument indicates that $\neg$ Max ranked over Max cannot be specific to vowels or consonants, suggesting the implausibility of Horwood's analysis even if anti-faithfulness theory is granted. The adequacy of Align-R(Root,C) as the markedness constraint operative here requires further scrutiny within a larger context of Koasati phonology and morphology, but in light of the typological prediction, my analysis with general Max is superior independently of the different theoretical conceptualization of subtractive morphology. 
I have discussed earlier that morphosyntactic categories are not inherent properties of bare stems. The fact that singular forms do not undergo any subtraction suggests that the morphosyntactic function singular corresponds to $\alpha$ in the Faith ${ }_{\alpha}$ » $\mathrm{RM} » \mathrm{Faith}_{\beta}$ schema, yielding $\mathrm{Max}_{\text {Singular }} » \mathrm{RM} » \operatorname{Max}_{\text {Plural }}$. In this context, consider another potential nonconcatenative morphology schema: $\mathrm{RM}_{\text {Plural }}$ » $\mathrm{Max}$ » $\mathrm{RM}_{\text {Singular }}$, where RM bears morphosyntactic markings instead of Max. This alternative shares the same tenet as the emergence of nonconcatenative morphology schema: some phonological action is required in the plural, but not in the singular. Although this alternative ranking appears to derive the same effect, it turns out that it suffers from a ranking paradox in the emergence of the unmarked cases like Koasati. For the examples in (1a), Align-R(Root,C) must be ranked over Max to explain rime subtraction in the plural formation, because otherwise only a final segment is expected to be clipped (i.e., $\mathrm{RM}_{\text {Plural }}$, Align-R(Root,C) » $\mathrm{Max} » \mathrm{RM}_{\text {Singular }}$ ), as shown in (8). On the other hand, to explain the singular forms in (1b), Align-R(Root,C) must be crucially ranked beneath $\operatorname{Max}$ (i.e., $\mathrm{RM}_{\text {Plural }}$ » $\operatorname{Max}$ » Align-R(Root,C), $\mathrm{RM}_{\text {Singular }}$ ), as delineated in (9). Obviously, this is a ranking paradox. This shows that this alternative indexation does not successfully capture the emergence of the unmarked effect. This is a serious problem because the emergence of the unmarked is not a language-specific property or specific characteristic of subtractive morphology.

\begin{tabular}{|ll||c:c|c|}
\hline & /pitaf-li-n/ $/$ Plural & $\mathrm{RM}_{\text {Plural }}$ & Align-R(Root,C) & Max \\
\hline \hline a. & pi.taf.lin & $* !$ & & \\
\hline b. & pi.ta.lin & & $* !$ & $*$ \\
\hline c. $\quad$ 吼 & pit.lin & & & $*$ \\
\hline
\end{tabular}




\begin{tabular}{|ll||c|c:c|}
\hline & /facoo-ka-n/Singular & Max & Align-R(Root,C) & $\mathrm{RM}_{\text {Singular }}$ \\
\hline \hline a. $\quad$ fa.coo.kan & & $*$ & $*$ \\
\hline b. & fac.kan & $* !$ & & \\
\hline
\end{tabular}

To solve this problem, Max could be divided into two constraints, each of which carries a morphosyntactic marking. This yields the following constraint ranking: $\mathrm{RM}_{\text {Plural }}$ » $\mathrm{Max}_{\text {Singular }}$ "Align-R(Root,C) 》 $\mathrm{Max}_{\text {Plural }}$ » $\mathrm{RM}_{\text {Singular. Notice, }}$ however, that this solution crucially takes advantage of indexed Max constraints, and given the generalized schema proposed in section 2.4 , there is no reason why RM must be indexed additionally. The generalized schema captures the emergence of the unmarked in nonconcatenative morphology without any index on RM. Thus, the alternative analysis contains analytical redundancy.

To close this section, I compare my analysis with several derivational approaches to Koasati subtractive morphology. First, Martin (1988) proposes that morphological truncation is a dissociation process. He analyzes the rime deletion examples in (1) as morphological rime dissociation. Under his analysis, the wordfinal rime is delinked from the syllable node (10a), and the onset of the final syllable is resyllabified as a coda consonant of the preceding syllable (10b). Finally, the segments affiliated with the delinked rime are deleted by the stray erasure convention (Steriade 1982; Itô 1986, 1989), as depicted in (10c). Martin crucially assumes that all rule types available in phonology (e.g., association, metathesis, and dissociation) are equally available in morphology as well (Martin 1988:236). His analysis generates correct outputs in Koasati but is theoretically not articulated sufficiently. Martin's proposal is rather descriptive since it does not explain why the final rime is subject to deletion. Additionally, as pointed out by Weeda (1992:225), his 
assumption crucially contains a duplication of the same operations in phonology and morphology. To avoid this problem, a system integrating phonology and morphology must be developed. Under the OT analysis developed above, morphological and phonological constraints are arranged in the form of ranked hierarchy, and therefore, morphology is not separated from phonology.
a. Rime delinking
b. Resyllabification
c. Stray erasure
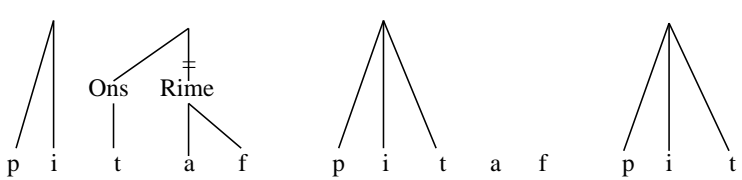

Lombardi and McCarthy (1991) provide a theoretically much more elaborated account for subtractive morphology. Their analysis crucially relies on prosodic circumscription theory (McCarthy and Prince 1990). This is theoretical advancement within the prosodic morphology program to delimit the domain of rule applications only to part of a morphological constituent. The idea behind this is that phonological rules are often sensitive only to a portion of an entire morphological constituent. Prosodic circumscription partitions it into phonologically affected and unaffected parts. It divides a base B into a parsing function $\Phi(\mathrm{C}, \mathrm{E})$ (where $\mathrm{C}$ is a prosodic constituent, and E represents an edge, either left or right), represented as B: $\Phi$ and into the residue of $\mathrm{B}$, expressed as $\mathrm{B} / \Phi$. The members of eligible prosodic units are a prosodic word, foot, syllable, or mora. A morphological operation recruits either B: $\Phi$ (positive prosodic circumscription) or $\mathrm{B} / \Phi$ (negative prosodic circumscription), and the unemployed portion remains unaffected by the operation at all. 
Building on prosodic circumscription theory, Lombardi and McCarthy (1991) propose that a delete operator is responsible for subtractive morphology. According to their analysis, rime deletion in Koasati pluralization is analyzed as follows: the final syllable is circumscribed (11a), and the circumscribed domain is deprosodified (11b). Resyllabification takes place subsequently (11c), and finally, prosodically unlicensed segments are deleted (11d) again due to the stray erasure convention.

a. Finally syllable circumscription

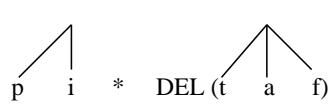

c. Resyllabification

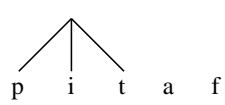

b. Deprosodification

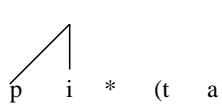

d. Stray erasure

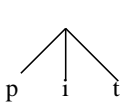

The delete operator approach was criticized in a cogent counter-argument by Weeda (1992): Lombardi and McCarthy's analysis makes a wrong empirical prediction with respect to the rustic girl's name formation in Japanese (Mester 1990; Poser 1990). First, consider the examples in (12) where their analysis yields correct results (Lombardi and McCarthy 1991:63).

$\begin{array}{ll}\text { Base Name } & \text { Rustic Girl's Name } \\ & \\ \text { Yuuko } & \text { o-Yuu } \\ \text { RaNko } & \text { o-RaN } \\ \text { Yukiko } & \text { o-Yuki } \\ \text { Kinue } & \text { o-Kinu } \\ \text { Midori } & \text { o-Mido }\end{array}$

Under Lombardi and McCarthy's analysis, the final syllable is first prosodically circumscribed, and the circumscribed syllable is deprosodified. Since no 
resyllabification takes place in these examples, the correct forms are obtained. (In Japanese, the licit codas are a placeless nasal, a place-linked nasal, or the first half of an obstruent geminate.) However, when a third syllable contains a nasal onset, their analysis generates a wrong output form. Consider [Sigeno] as a base name. The first two steps are the same as the examples in (12). Crucially, the nasal onset of the third syllable can be resyllabified as a coda consonant of the second syllable. The eventual output would be [o-SigeN], contrary to fact (i.e., [o-Sige]). Besides the criticism by Weeda, the delete operator analysis predicts that the output is larger than two syllables if the input is more than three syllables long because the delete operator targets only a final syllable. However, all rustic girl's names are stringently of bimoraic size, except the initial honorific prefix $o$-. Many Japanese female first names consist of four syllables, and therefore, Lombardi and McCarthy's analysis based upon prosodic circumscription is empirically flawed.

In this connection, I review Weeda (1992) as well. He develops an analysis called circumscription-plus-mapping (cf. Mester 1990). Along with Lombardi and McCarthy's proposal, this analysis is also framed in prosodic circumscription theory. The idea is that a base form undergoes certain circumscription, and the circumscribed segments are further mapped onto a template. The elements which do not fit in with the template are subsequently deleted by the stray erasure convention. A series of these operations is exemplified in (13) with Papago perfectives where a final syllable is elided (Hale 1965; Pranka 1983; Zepeda 1983; Lombardi and McCarthy 1991; Hill and Zepeda 1992; Weeda 1992; Golston 1996; Fitzgerald 1997; Horwood 1999). First, a syllabic trochee is circumscribed at the right edge of the base (13a). Second, the circumscribed domain is picked up, saving the residue (13b). Third, a 
monosyllabic template is mapped onto the left edge of the result of (13b), as indicated in (13c). Fourth, the saved residue is put back (13d), and finally, syllabically unlicensed elements are elided by stray erasure (13e).

a. Circumscription b. Saving and Picking

c. Template mapping
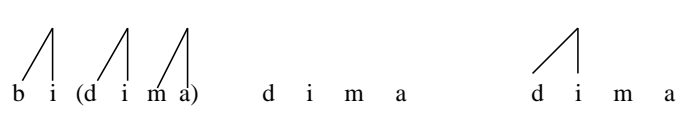

d. Return residue

e. Stray erasure
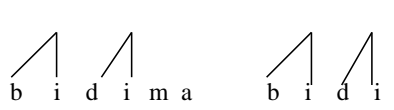

Weeda (1992) argues that this alternative analysis is superior to any approaches reviewed above. However, I claim that it is also unsatisfactory. My argument comes from an independent consideration against prosodic circumscription theory. As outlined above, this theory is designed to delimit the domain of morphological operations. It plays a central role in prosodic morphology and has been applied to infixation and reduplication as well as truncation. The presence of negative prosodic circumscription, however, makes a serious undesirable prediction. Suppose that there is a language $\mathrm{L}$ in which a disyllabic foot is circumscribed at the left periphery of a base. The result is that the phonological size of the residue varies depending on the base size, as delineated schematically in (14a, b, c). Given negative prosodic circumscription and productive partial reduplication across languages, a consequence is that $\mathrm{L}$ potentially allows for reduplication where the residue of the disyllabic foot prosodic circumscription serves as the reduplicant, as shown in (14a', $\left.b^{\prime}, c^{\prime}\right)$, where the reduplicants are underscored. No such language exists, however, to 
the best of my knowledge. This shows that prosodic circumscription theory generates unobserved types of reduplication. The theory is hence over-powerful.

Trisyllabic base: Quadrisyllabic base: Pentasyllabic base:
a. $\quad\left[\sigma_{1} \sigma_{2}\right] \sigma_{3}$
b. $\quad\left[\sigma_{1} \sigma_{2}\right] \sigma_{3} \sigma_{4}$
a'. $\quad \sigma_{1} \sigma_{2} \sigma_{3}-\underline{\sigma}_{3}$
c. $\quad\left[\sigma_{1} \sigma_{2}\right] \sigma_{3} \sigma_{4} \sigma_{5} \quad c^{\prime}$.
$\sigma_{1} \sigma_{2} \sigma_{3} \sigma_{4}-\underline{\sigma}_{3} \underline{\sigma}_{4}$
$\sigma_{1} \sigma_{2} \sigma_{3} \sigma_{4} \sigma_{5}-\underline{\sigma}_{3} \underline{\sigma}_{4} \underline{\sigma}_{5}$

Furthermore, McCarthy (2000a) develops conceptual arguments against prosodic circumscription theory. Most importantly, he argues that this theory is a device special to prosodic morphology, so it does not make any deep connections clear between prosodically regulated morphological operations such as affixation, truncation, reduplication etc. and other phonological phenomena. He claims that prosodic faithfulness takes the place of prosodic circumscription, the former being amply motivated for independent reasons. By eliminating prosodic circumscription designed to capture a very parochial range of data, a higher level of linguistic explanation can be achieved. These empirical and conceptual arguments together undermine the theoretical status of prosodic circumscription theory (see also Prince and Smolensky 1993:chapter 4 and McCarthy and Prince 1993b:chapter 7 for arguments against prosodic circumscription in the context of infixation).

Since Weeda's (1992) circumscription-plus-mapping crucially depends on prosodic circumscription theory, the argumentation above poses a worry that his proposal is based on a mistaken premise. To the extent that my argument against prosodic circumscription theory is effective, the plausibility of circumscription-plusmapping (as well as the delete operator analysis of Lombardi and McCarthy 1991) becomes dubious. No superficial fix ameliorates the situation here since the problem directly stems from the fundamental theoretical background on which Weeda's analysis is constructed. 


\subsubsection{Lardil}

Lardil, an Australian language spoken on Morningthon Island in the Gulf of Carpenteria, is one of the most intensively and extensively studied languages among those which exhibit subtractive morphology. Lardil presents subtractive morphology in the nominative formation. Earlier contributions include Hale (1973), Itô (1986), Wilkinson (1988), Weeda (1992), Prince and Smolensky (1993), Blevins (1997), Horwood (1999) and Kurisu (1999). Examples are given in (15), where retroflex segments are denoted by capital letters.

\section{Stem}

a. yalulu yiliyili karikari kankari mayara kamputa wiwala

b. Tant ${ }^{y}$ irk wanalk naluk kuRkan peRen wunkunun kurpuRun Tankun

c. wulunka

puTuka murkunima yukarpa yaakuwa TipiTipi murkunima pulumunitami jawunawu munkumunku $t^{y} u m p u t^{y} u m p u$

\section{Nominative Gloss}

yalul

yiliyil karikar kankar mayar kamput wiwal

Tant ${ }^{y}$ ir wanal nalu kuRka peRe wunkunu kurpuRu

Tanku

wulun
puTu
murkuni
yukar
yaaku
TipiTi
murkuni
pulumunita
nawuna
mugkumu
t'umput'u

wulun murkuni yukar yaaku TipiTi murkuni pulumunita nawuna $t^{y} u^{y}$ put $^{y} u$ flame oyster butter-fish father's father rainbow pandoanas nuts bush mango

hip
boomerang
story
pandja
vagina
queen-fish
lancewood
oyster found in reefs
fruit
short
nullah
husband
blue-tongued lizard
Rock-cod sp.
fighting stick
young female dugong
termite
wooden axe
dragonfly


As exemplified in $(15 a, b)$, this word formation is characterized as final segment deletion. But, as will be discussed shortly, final consonant deletion in (15b) is independently motivated in phonological terms. Lardil nominative formation is analyzed as apocope by Hale (1973) and Itô (1986) and as aggressive extrametricality by Wilkinson (1988) respectively. Their derivational analyses are not satisfactory, however, because final vowel deletion does not follow from any principled motivation. Thus, the first question is how final vowel deletion is explained in a firmly grounded manner.

A second problem concerns the existence of examples which apparently do not conform to the descriptive generalization that only a final vowel is subject to deletion. That is, (15c) recruits final (C)CV deletion. Nevertheless, a close examination reveals that this generalization is correct. My analysis below is essentially built upon Prince and Smolensky (1993:chapter 7). But, as will be discussed shortly, my analysis departs from theirs in an important respect. In addition, I demonstrate in this section that RM is a violable constraint. This is naturally expected given that all universal constraints are potentially violable and that $\mathrm{RM}$ is one of them.

First, consider (15a). These examples involve deletion of a stem-final vowel. Given the subtractive morphology schema (i.e., $\operatorname{Max}_{\alpha} » \mathrm{RM} » \mathrm{Max}_{\beta}$ ), they receive a straightforward explanation, crucially RM dominating $\operatorname{Max}_{\text {Nominative, }}$ as shown in (16). This analysis provides an immediate answer to the first question raised above: how does final vowel deletion receive a firmly grounded theoretical account? The nominative morpheme does not have any fixed segmentism or prosody, and therefore, its phonological content is empty. The stem must be affected under the pressure of 
RM. Given RM » $\operatorname{Max}_{\text {Nominative, }}$ a final vowel must be sacrificed, assuming that the word-initial portion is protected by a positional faithfulness constraint (Beckman 1995, 1997).

\begin{tabular}{|ll||c|c|}
\hline & /yalulu/Nominative & $\mathrm{RM}$ & $\mathrm{Max}_{\text {Nominative }}$ \\
\hline \hline a. & yalulu & $* !$ & \\
\hline b. & yalul & & $*$ \\
\hline c. & yalu & & $* * !$ \\
\hline d. & yal & & $* * ! *$ \\
\hline
\end{tabular}

Wilkinson's (1988) approach to final vowel deletion is similar to the morphological dissociation analysis of Martin (1988). Her essential proposal is that a final vowel becomes extrametrical when a stem is not inflected: aggressive extrametricality. The extrametricalized segment is not prosodically licensed, and therefore, is eventually deleted by the stray erasure convention. But this analysis is clearly brute-forced because aggressive extrametricality is unpredictable with respect to regular extrametricality. Her analysis is also vulnerable to the same conceptual problem as the morphological dissociation approach. Although it might appear that final vowel deletion follows from stray erasure, this analysis simply stipulates that the elided element does not receive any prosodic licensing. Aggressive extrametricality is also untenable for the same reason as that led us to reject Martin's (1988) dissociation analysis. In the proposed OT analysis, final vowel deletion occurs as the best phonological exponence of the phonologically empty nominative morpheme.

My analysis strikingly differs from Prince and Smolensky's (1993:chapter 7) too. They posit Free-V which requires underparsing of word-final vowels (Prince and 
Smolensky 1993:101). They crucially assume that Free-V is operative only in the nominative. They try to justify this assumption by arguing that virtually the same constraint can be applied to Estonian morphology. This idea has at least two serious problems. First, recall from the typology of subtractive morphology that nominatives are not the only morphosyntactic category in which final vowel elision occurs across languages. Second, Itô and Mester (1999b) demonstrate that morphological relativization of a markedness constraint results in the back-copying problem discussed by Prince (1996), Spaelti (1997), and McCarthy and Prince (1999). Itô and Mester (1999b) draw a conclusion that only faithfulness constraints are qualified for the sensitivity to particular morphological information. Free-V is clearly a markedness constraint, however. These two points significantly undermine the plausibility of the universal status of Free-V (see Horwood 1999 for an argument against Free-V arising from the different theoretical design of containment theory and correspondence theory). In my analysis, it is faithfulness constraints that bear morphologically idiosyncratic information. Moreover, all patterns of subtractive morphology are reduced to the general subtractive morphology schema (i.e., $\operatorname{Max}_{\alpha}$ » $\mathrm{RM} » \mathrm{Max}_{\beta}$ ), or more generally, to the emergence of nonconcatenative morphology schema (i.e., Faith ${ }_{\alpha} »$ RM » Faith ${ }_{\beta}$ ) which encompasses the whole nonconatenative morphology. My explanation has broader perspectives and wider empirical coverage.

I discussed in section 2.5 that the nominative formation at issue presents a serious empirical challenge to anti-faithfulness theory. Anti-faithfulness theory is transderivational in the sense of Benua (1997), meaning that outputs are restricted to independent words actually attested on the surface. Since Lardil stems never surface on their own, nominatives simply cannot have their bases from which they are 
derived under the system of anti-faithfulness. As spelled out in section 2.3, two output forms are compared to evaluate their (non-)identity for the computation of the satisfaction/violation of RM under RMT, but the output of a bare stem does not have to be a real output in the language. The minimal requirement is that they are at least possible outputs besting phonological factors independently needed in the language. This difference is significant because RMT can deal with the nominative formation in Lardil but anti-faithfulness theory cannot.

Let us turn to $(15 \mathrm{~b}, \mathrm{c})$. The question is whether these cases are simply other variants of morphological subtraction or phonologically conditioned. Itô (1986) and Wilkinson (1988) thoroughly investigate permissible syllable structures in the language and conclude that only coronal consonants are allowed word-finally. Wordmedially, consonants of other places of articulation are properly licensed only when the place is shared by the immediately following onset. Itô (1986:88) formulates these coda restrictions as a coda condition that prohibits any non-place-linked noncoronal consonants in the syllable coda. Looking at (15b) with this in mind, remarkable generalization emerges: a final consonant is non-coronal, and hence, faithful parsing of the consonant gives rise to an impermissible syllable structure in Lardil. Adopting the coda condition (henceforth, CodaCond) as a collection of constraints deriving the descriptive generalization, final consonantal deletion is not due to the requirement of morphological subtraction proper. Given this, the data in (15c) receive a proper understanding. Final vowel deletion is regulated by subtractive morphology as in (15a), and further consonant deletion is employed to erase offending coda consonants in terms of CodaCond. Thus, only final vowel elision is morphologically governed, other deletion being required for phonological reasons. 
This analysis is exemplified in the tableau in (17). The dominating status of CodaCond and RM over Max $_{\text {Nominative }}$ is essential. First, candidate (17a) is ruled out by RM, and candidate (17b) is eliminated by its fatal violation of CodaCond. The decision between (17c) and (17d) is passed on to low ranked $\operatorname{Max}_{\text {Nominative }}$. Since a coronal is allowed in the syllable coda, its deletion meaninglessly increases $\operatorname{Max}_{\text {Nominative }}$ violations. (NoCoda is ranked below $\operatorname{Max}_{\text {Nominative }}$ ) The desired output is correctly obtained. The cases where two consonants are deleted (i.e., /munkumunku/ and $/ \mathrm{t}^{\mathrm{y}} u \mathrm{mput} \mathrm{t}^{\mathrm{y}} \mathrm{umpu} /$ ) are also accounted for in the same manner: only one consonant deletion is not sufficient for the satisfaction of high ranked CodaCond.

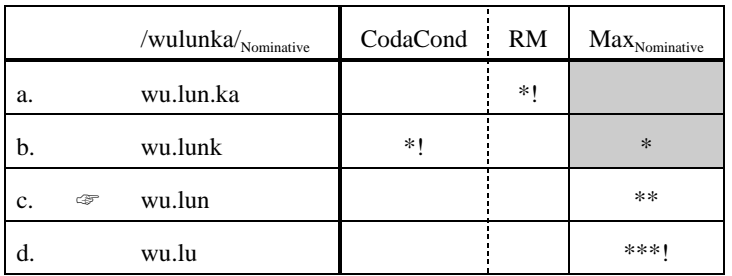

The relative hierarchy between CodaCond and RM might seem irrelevant, but indeed, a strict ranking can be established. The examples in (15b) contribute to this decision. Under RMT, the output is compared with the output of the bare stem when a stem serves as the input. All requirements of regular phonology are imposed on the bare stem output as well. This means that the output of the bare stem /Tant ${ }^{\mathrm{y}}$ irk/, for example, is [Tant ${ }^{\mathrm{y}}$ ir] since consonants violating CodaCond are subject to deletion. Given the fact that the nominative form of $/ \operatorname{Tant}^{\mathrm{y}} \mathrm{irk} /_{\text {Nominative }}$ is also [Tant ${ }^{\mathrm{y}} \mathrm{ir}$ ], the nominative forms in (15b) indeed violate RM. As demonstrated in (18), this observation leads to justify CodaCond » RM. This is a point closely related to the 
nature of the nominative formation. Morphological subtraction is only final vowel deletion, but every consonant deletion is phonological. This in turn suggests that word-final consonant deletion is neither a deletion process to satisfy RM nor a conspiracy effect to satisfy CodaCond and RM simultaneously through a single phonological operation. Rather consonant deletion is purely phonological.

\begin{tabular}{|c|c|c|c|c|}
\hline & /Tant ${ }^{\mathrm{y} i r k} /_{\text {Nominative }}$ & CodaCond & $\mathrm{RM}$ & $\operatorname{Max}_{\text {Nominative }}$ \\
\hline a. & Tan. $t^{\mathrm{y}}$ irk & $* !$ & & \\
\hline b. & Tan. $\mathrm{t}^{\mathrm{y}}$ ir & & $*$ & $*$ \\
\hline
\end{tabular}

In the plural formation in Koasati, RM is always satisfied, at least as far as the data we considered are concerned, but RM is violated here. This is a naturally expected result given that RM is a member of universal constraints, conforming to the elementary assumption of OT that constraints are potentially all violable. But this is not the only context in which RM fails to be satisfied. There are two more circumstances under which RM is violated. One such case is when a stem ends in a coronal consonant, as shown in (19).

\begin{tabular}{|c|c|c|}
\hline Stem & Nominative & Gloss \\
\hline thungal & thunal & tree \\
\hline kentapal & kentapal & dugong \\
\hline kethar & kethar & river \\
\hline$t^{y}$ umur & $\mathrm{t}^{\mathrm{y}}$ umur & coolimon \\
\hline miyaR & miyaR & spear \\
\hline yaRput & yaRput & snake, bird \\
\hline jampit & gampit & humpy \\
\hline kalt ${ }^{y}$ it & kalt ${ }^{y}$ it & urine \\
\hline yaraman & yaraman & horse \\
\hline Tupalan & Tupalan & road \\
\hline pirnen & pirnen & woman \\
\hline $\mathrm{kaN} \mathrm{t}^{\mathrm{y}}$ in & $\mathrm{kaN}^{\mathrm{t}} \mathrm{t}^{\mathrm{y}}$ in & wallaby \\
\hline maan & maan & spear \\
\hline
\end{tabular}


These stems are closed by a coronal consonant, so CodaCond is satisfied. Given $\mathrm{RM} » \mathrm{Max}_{\text {Nominative }}$, however, final consonant deletion is expected, contrary to fact. As an operative constraint, I assume Anchor- $\left.\mathrm{R}(\mathrm{C}]_{\mathrm{Stem}}, \mathrm{Syll}\right)$, an anchoring constraint of the slightly modified alignment constraint proposed by Prince and Smolensky (1993:101). Anchor-R(C $]_{\text {Stem }}$,Syll) inclusive, the four constraints so far are ranked as follows: CodaCond » Anchor- $\left.\mathrm{R}(\mathrm{C}]_{\mathrm{Stem}}, \mathrm{Syll}\right)$ » $\mathrm{RM}$ » $\mathrm{Max}_{\text {Nominative }}$. CodaCond » Anchor- $\left.\mathrm{R}(\mathrm{C}]_{\mathrm{Stem}}, \mathrm{Syll}\right)$ is justified by the examples in (15b). As demonstrated in (20), Anchor-R(C $]_{\text {Stem }}$,Syll) crucially prevents morphological subtraction in the examples in (19).

\begin{tabular}{|c|c|c|c|c|c|}
\hline & & /thungl/ ${ }_{\text {Nominative }}$ & Anchor-R(C $]_{\text {Stem }}$, Syll $)$ & RM & $\operatorname{Max}_{\text {Nominative }}$ \\
\hline a. & $\operatorname{sis}$ & thu.nal & & $*$ & \\
\hline $\mathrm{b}$ & & thu.na & $* !$ & & * \\
\hline
\end{tabular}

Another case where RM is violated is due to word minimality. Wilkinson (1988) argues that Lardil obeys a bimoraic minimal word requirement. Since coda consonants are not weight bearing units in Lardil as in many other Australian languages, minimal words are disyllabic. As shown in (21a), when the base stem of the nominative is disyllabic, no vowel deletion takes place. That word minimality is operative is further corroborated by the fact that epenthesis takes place in the nominative formation when a stem is subminimal, as in (21b). Obviously, Anchor$\mathrm{R}(\mathrm{C}]_{\text {Stem }}$,Syll $)$ does not play any role here. What is responsible here is a minimality constraint (i.e., MinWd) which forces a prosodic word to be at least of bimoraic size. MinWd, a set of constraints yielding the minimality effect, evidently outranks RM. Thus, $/ \mathrm{kela} / /_{\text {Nominative }}$, for example, is realized as $[\mathrm{kela}]_{\text {Nominative }}$ with no deletion. MinWd 
is undominated in Lardil, and RM is ranked beneath this phonological markedness constraint here, revealing another instance of RM violability. This is demonstrated in (22) below.

\begin{tabular}{|c|c|c|}
\hline Stem & Nominative & Gloss \\
\hline kela & kela & beach \\
\hline paRna & paRna & stone \\
\hline wanka & wanka & arm \\
\hline kugka & kunka & groin \\
\hline Targka & Targka & barracuda \\
\hline guka & nuka & water \\
\hline gawa & jawa & $\operatorname{dog}$ \\
\hline kaTa & kaTa & child (of a woman) \\
\hline gura & pura & forehead \\
\hline muNa & muNa & elbow \\
\hline wik & wika & shade \\
\hline $\mathrm{maR}$ & maR.Ta & hand \\
\hline
\end{tabular}

\begin{tabular}{|c|c|c|c|c|}
\hline & & /kela/ Nominative & MinWd & RM \\
\hline a. & $\Leftrightarrow \notin$ & kela & & * \\
\hline $\mathrm{b}$ & & kel & $* !$ & \\
\hline
\end{tabular}

The analysis above finishes the exposition of subtractive morphology in Lardil, but to complete the whole picture, let us consider two types of epenthesis found in (21b). Of principal interest here is the contrast between vowel epenthesis and $\mathrm{CV}$ epenthesis found in the two examples. However, this is a direct consequence of the constraints developed above, especially Anchor- $\left.\mathrm{R}(\mathrm{C}]_{\mathrm{Stem}}, \mathrm{Syll}\right)$ (cf. Prince and Smolensky 1993:111-113). Assuming that Dep (a constraint banning insertion) is ranked beneath Anchor- $\mathrm{R}(\mathrm{C}]_{\text {Stem }}$, Syll), two tableaux are given below, each for the two examples in (21b). First, in (23) where the input is closed by a non-coronal consonant, $\mathrm{CV}$ epenthesis is not appropriate. This is because, by epenthesizing a 
consonant in addition to a vowel, the stem-final consonant is deprived of the possibility of occupying the onset of a second syllable to avoid violating CodaCond. The optimal candidate satisfies CodaCond by positioning the stem-final consonant in the syllable onset, although it is penalized for its violation on Anchor- $\left.\mathrm{R}(\mathrm{C}]_{\mathrm{Stem}}, \mathrm{Syll}\right)$. By contrast, in (24), the stem-final consonant is right-anchored to a syllable. Since CodaCond does not matter here, Anchor- $\mathrm{R}(\mathrm{C}]_{\text {stem }}$,Syll) mandates for the optimal output form an extra violation of Dep. This option is not available in (23) because non-coronal coda consonants infringe on CodaCond. This shows that CodaCond and Anchor- $\mathrm{R}(\mathrm{C}]_{\text {Stem }}$, Syll $)$ not simply regulate morphological subtraction but also play an essential role in the decision of optimal epenthesis.

\begin{tabular}{|ll||c|c|c|}
\hline & $/$ wik/ $/_{\text {Nominative }}$ & CodaCond & Anchor-R(C $]_{\text {Stem }}$, Syll $)$ & Dep \\
\hline \hline a. $\quad$ wi.ka & & $*$ & $*$ \\
\hline b. & wik.Ta & $* !$ & & $* *$ \\
\hline
\end{tabular}

\begin{tabular}{|ll||c|c|c|}
\hline & $/ \mathrm{maR} /{ }_{\text {Nominative }}$ & CodaCond & Anchor-R(C $]_{\text {Stem }}$, Syll $)$ & Dep \\
\hline a. & ma.Ra & & $* !$ & $*$ \\
\hline b. $\quad$ maR.Ta & & & $* *$ \\
\hline
\end{tabular}

Summarizing the above, I analyzed subtractive morphology involved in the nominative formation in Lardil. A couple of final remarks are in order. First, RM is a violable constraint, and therefore, a violation of RM is necessary when a higher ranked faithfulness or markedness constraint must be fulfilled at the expense of RM violations. As can be seen from the overall ranking of the relevant constraints given in (25), RM is dominated by CodaCond, Anchor- $\left.\mathrm{R}(\mathrm{C}]_{\mathrm{Stem}}, \mathrm{Syll}\right)$, and MinWd. Second, 
the analysis unifies morphological and phonological factors in the form of a single constraint ranking. Given the analysis here centered on RM, a single constraint ranking is responsible for both morphological subtraction proper as well as for various associated phonological processes. This avoids the duplication problem arising from positing the same operations for phonology and morphology independently. Furthermore, under RMT where the output is compared with the output of the relevant bare stem, cases like the nominative formation in Lardil can be easily accommodated because the output of the bare stem does not have to be an actually existing independent word in the language. This is a crucial point that empirically distinguishes RMT and anti-faithfulness theory. RMT utilizing the notion of possible outputs is preferred.

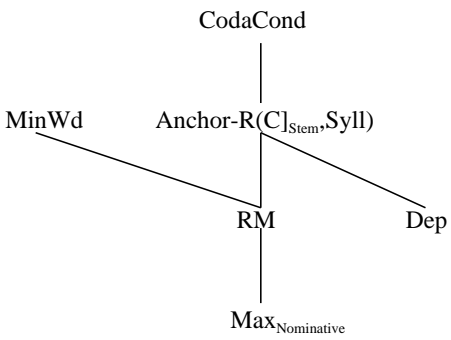

\subsubsection{Icelandic}

In this section, I examine an under-application effect of segmental deletion involved in Icelandic deverbal noun formation (for the relevant work, see Orešnik 1972, 1978ab; Arnason 1980; Kiparsky 1984; Itô 1986; Benua 1995). I provide an analysis 
built on RMT and compare it with Benua's (1995) analysis. The relevant data are provided in (26). The crucial observation is that deverbal nouns violate SonCon that prohibits a sequence of coda consonants with rising sonority.

$\begin{array}{llll}\text { Infinitive } & \text { Gloss } & \text { Deverbal Noun } & \text { Gloss } \\ \text { klifra } & \text { climb } & \text { klifr } & \text { climbing } \\ \text { kumra } & \text { bleat } & \text { kumr } & \text { bleating } \\ \text { grenja } & \text { cry } & \text { grenj } & \text { crying } \\ \text { söötra } & \text { sip } & \text { söötr } & \text { sipping } \\ \text { puukra } & \text { conceal } & \text { puukr } & \text { concealment } \\ \text { kjöökra } & \text { wail } & \text { kjöökr } & \text { wailing }\end{array}$

Under the current system that morphosyntactic categories are not inherent properties of stems, a potential analysis is to consider that infinitives and deverbal nouns are derived from bare stems respectively. But there is strong evidence in this case to show that infinitives are indeed the bases of deverbal noun forms. In Icelandic, long vowels are permitted only in stressed open syllables in general, but some of the deverbal nouns in (26) show that long vowels are permitted even in a closed syllable. This seemingly inconsistent fact is directly captured by assuming that deverbal nouns are derived from infinitives such that a vowel transfer effect is due to the faithfulness between infinitive and deverbal noun forms. Given the fact that vowel deletion takes place in the deverbal noun formation, RM outranks $\mathrm{Max}_{\mathrm{DVN}}$. Furthermore, no consonant deletion occurs to avoid violating SonCon, meaning that $\operatorname{Max}_{\mathrm{DVN}}$ in turn dominates SonCon. This analysis is demonstrated in (27).

\begin{tabular}{|ll||c|c|c|}
\hline & \multicolumn{1}{|c||}{$/$ klifra/ $_{\text {DVN }}$} & $\mathrm{RM}$ & $\operatorname{Max}_{\mathrm{DVN}}$ & SonCon \\
\hline \hline a. & klifra & $* !$ & & \\
\hline b. & klifr & & $*$ & $*$ \\
\hline c. & klif & & $* * !$ & \\
\hline
\end{tabular}


An important point has to do with the way that RM is evaluated. As proposed in section 2.3, RM compares the input and the output when the input is already an independent output form. Since infinitive forms appear as independent words, RM compares the phonological identity/dissimilarity of an infinitive and the derived deverbal noun forms. As schematized in (28), infinitive forms are derived from bare forms by adding /-a/ (except for a handful of exceptions such as [ske] 'happen' and [nau] 'get/fetch'). This means that RM compares the output form of the bare form and output candidates when the infinitive forms are evaluated. The fact that infinitive forms obey SonCon suggests that $\mathrm{Max}_{\text {Inf. }}$ is ranked below SonCon, yielding RM » $\operatorname{Max}_{\text {DVN }}$ »SonCon » $\operatorname{Max}_{\text {Inf. }}$ Although RM outranks $\operatorname{Max}_{\text {Inf., no morphological }}$ subtraction takes place in infinitives since faithful parsing of the affixal material suffices to satisfy RM.

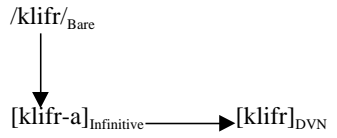

Now, consider the examples provided in (29) (Benua 1995:90). The crucial observation is that the final consonant of a stem violating SonCon is subject to deletion when it is not followed by any suffix: the final /j/ of the stem /bylj/ undergoes elision in accusative/dative singulars whereas the final consonant appears when a vowel-initial suffix follows the stem.

(29) Stem: /bylj/ 'snowstorm'

$\begin{array}{llll}\text { Accusative singular: } & \text { byl } & \text { Accusative plural: } & \text { bylj-i } \\ \text { Dative singular: } & \text { byl } & \text { Dative plural: } & \text { bylj-um } \\ \text { Genitive singular: } & \text { byls/bylj-ar } & \text { Genitive plural: } & \text { bylj-a }\end{array}$


Focusing on accusative/dative singulars, final consonant deletion is due to SonCon and therefore, the pertinent Max is dominated by it. I have discussed that faithfulness constraints are relativized with respect to various morphosyntactic categories. In Icelandic, only deverbal nouns violate SonCon, but given that $\operatorname{Max}_{\mathrm{DVN}}$ is ranked over SonCon, we can postulate general Max-IO below SonCon rather than Max specific to accusative/dative singulars. Since $\operatorname{Max}_{\mathrm{DVN}}$ » SonCon is a Paninian ranking (i.e., a more specific constraint is ranked over a markedness constraint), assuming general Max does not impinge upon the result of deverbal noun formation. This situation is different from other examples of nonconcatenative morphology because anti-Paninian ranking is crucial in those cases. An important lesson here is that faithfulness constraints are relativized with respect to morphosyntactic categories only when it yields an anti-Paninian ranking.

This consideration is crucial in the analysis of accusative/dative singulars in Icelandic. Given a bare stem as the input, /bylj/ serves as the input of $[\text { byl }]_{\text {Acc./Dat.Sg. }}$. Given SonCon » Max, the overall ranking established so far is RM » SonCon » Max. The crucial issue here is that this ranking predicts morphological subtraction whether SonCon is satisfied or not, contrary to fact, since RM dominates general Max. Final consonant deletion in [byl] is purely phonological, and therefore, this is an undesired prediction. I propose that there is an output-output correspondence constraint to block this effect. Given SonCon » Max, the output of the bare stem /bylj/ is [byl]. The pertinent output-output constraint is thus Dep-OO, where the two outputs are a bare stem output and an accusative/dative singular form. The calculation of the output of the bare stem depends upon the ranking SonCon » Max since Icelandic has a general Max constraint. As demonstrated in (30), this analysis correctly generates 
[byl]. Thus, morphologically conditioned deletion is blocked by virtue of the fact that Faith-OO outranks RM. No deletion takes place in the accusative/dative singular formation when the bare stem contains a complex coda which obeys SonCon, Max$\mathrm{OO}$ needs to be ranked over RM as well. This output-output correspondence crucially takes advantage of the output of a bare stem, but this is not extra machinery because the notion of bare stem outputs is independently motivated in section 2.3 .

\begin{tabular}{|ll||c|c|c|c|}
\hline & /bylj/ $/_{\text {Acc.Jat.Sg. }}$ & Dep-OO & RM & SonCon & Max-IO \\
\hline \hline a. & bylj & $* !$ & & $*$ & \\
\hline b. & byl & & $*$ & & $*$ \\
\hline
\end{tabular}

Benua (1995) also employs constraint-based OT, but her analysis is different from mine. She proposes that transderivational correspondence plays an essential role. Following the related earlier work, Benua assumes that deverbal nouns are derived from corresponding infinitive forms, as I did for my analysis above. (26) shows that a complex coda with rising sonority is permitted in deverbal nouns, and therefore, Max-OO outranks SonCon which prohibits codas with rising sonority, as demonstrated in (31). Her analysis is crucially different from mine in that faithfulness constraints are not sensitive to morphosyntactic information.

\begin{tabular}{|c|c|c|c|c|}
\hline & & /klifra/ ${ }_{\text {DVN }}$ & Max-OO & SonCon \\
\hline a. & $0 \notin 8$ & klifr & * & $*$ \\
\hline b & & klif & $* * !$ & \\
\hline
\end{tabular}

On the other hand, SonCon plays a role as the driving force of consonant deletion in the input-output dimension, as in (29). This observation motivates another 
ranking: SonCon » Max-IO, as shown in (32). By transitivity, Max-OO » SonCon » Max-IO follows. In deverbal nouns, where output-output correspondence is active, consonant deletion under-applies because Max-OO is ranked higher than SonCon. In effect, Benua (1997:99) proposes Faith-OO » Markedness » Faith-IO as the general ranking yielding under-applications.

\begin{tabular}{|ll||c|c|}
\hline & /bylj/ $/_{\text {Acc./Dat.Sg. }}$ & SonCon & Max-IO \\
\hline \hline a. & bylj & $* !$ & \\
\hline b. $\quad$ b & byl & & $*$ \\
\hline
\end{tabular}

Benua's analysis has a fundamental problem, however. The difficulty resides in the lack of an account of the reason why truncation takes place in the first place. Notice that no truncation-inducing constraint can be ranked over Faith-IO because it would require infinitive forms to undergo truncation, contrary to fact. By contrast, such a constraint must be ranked above Max-OO to ensure final vowel deletion in deverbal nouns. But given Faith-OO » Faith-IO, no such ranking is logically possible. This proves that it is impossible to obtain the truncation effect only in the deverbal noun formation from the given constraint ranking. The same problem arises in cases of over-application because Faith-OO must dominate Faith-IO.

Benua assumes that at least one segment must be elided in the truncation mode by incorporating the morpheme TRUNC in the underlying representation of deverbal nouns, as is evident from the fact that she does not take [klifra] into consideration. This candidate incurs no violation of Max-OO, so it is more harmonic than $[\mathrm{klifr}]$. For this approach to work, such phonologically empty but processspecific morphemes must be well motivated, but as I argued in section 2.4 , the 
postulation of TRUNC runs into a serious empirical problem from a typological point of view. Moreover, as pointed out by Sanders (2000), Benua's approach is a recessive move in OT in the sense of serious input dependency. We need an explanation for subtractive morphology which relies on constraint interactions rather than on such a dubious morpheme.

My analysis centered on RM can avoid the problems encountered by Benua's transderivational approach. First, morphological subtraction is motivated by the strength of RM ranked over $\mathrm{Max}_{\mathrm{DVN}}$, but no consonantal deletion occurs to avoid a violation of SonCon in deverbal nouns. This entails that the failure of consonant deletion to satisfy SonCon is rather an issue of faithfulness priority. Second, my analysis avoids coming across the ranking paradox of the transderivational analysis by making faithfulness constraints be sensitive to morphosyntactic categories. Generalizing the analysis proposed here, so-called under-application observed in morphological truncation follows from the ranking of $\mathrm{RM} » \mathrm{Max}_{-}-\mathrm{IO}_{\alpha}$ » Markedness » Max- $-\mathrm{IO}_{\beta}$. Morphosyntactic category $\beta$ (i.e., infinitives in Icelandic) is derived from bare forms and obeys the relevant phonological markedness constraint. On the other hand, morphosyntactic category $\alpha$ (deverbal nouns in Icelandic), whose phonological content is null, comes from morphosyntactic category $\beta$. Since forms affiliated with morphosyntactic category $\beta$ are independent outputs, $\mathrm{Max}_{-} \mathrm{IO}_{\alpha}$ is virtually an outputoutput constraint. Given the lack of phonological substance of morpheme $\alpha$ and RM » $\mathrm{Max}-\mathrm{IO}_{\alpha}$, minimal morphological subtraction is required, but no further deletion takes place because of Max- $\mathrm{IO}_{\alpha}$ » Markedness even if the minimal segmental elision results in a violation of the relevant markedness constraint. The fact that the category $\beta$ does not undergo morphological truncation is explained by Dep- $\mathrm{O}_{\text {Bare }} \mathrm{O}_{\beta}$ » RM. 


\subsubsection{Hessian German}

Hessian German, a variety of German dialects, exhibits subtractive morphology in the plural formation. Plurals are not exclusively expressed by segmental deletion, other variations being umlaut, suffixation (i.e., $-r,-n$, and $-\theta$ ), and zero morphology (i.e., no overt phonological manifestation). As in Standard German, where several plural morphemic variations are attested, the selection of these allomorphs is not entirely predictable, and therefore, it is rather lexically idiosyncratic. Golston and Wiese (1995) analyze the alternation between zero and subtractive allomorph within the framework of OT. A further theoretical contribution is made by Horwood (1999) within the framework of anti-faithfulness theory. I focus here on the zero $\approx$ subtractive allomorphemic alternation and analyze it within the system developed thus far. I also critically assess Golston and Wiese (1995) and Horwood (1999).

Relevant examples are provided in (33). All the examples were excerpted from Golston and Wiese (1995:146-148). As in (33a), subtractive morphology is characterized as final consonant deletion. It takes place if the final two segments of a base are homorganic, assuming that vowels have a dorsal feature specification. By contrast, no segmental deletion occurs if the final two segments are heterorganic, as shown in (33b). Again, the deletion blockage in cases where the second last segment is a vowel is straightforwardly accommodated by assuming that vowels carry a dorsal specification. This observation indicates that the zero and subtractive allomorphs are in complementary distribution, supporting the idea that their distributions are phonologically definable. Although a change of vowel quality is observed in these examples, I abstract away from it in the following discussion. This would be a safe simplification given that it does not occur in all examples and that the change is quite 
irregular. Golston and Wiese (1995) argue that it is not just the place of articulation that is relevant to explain the (non-)occurrence of word-final consonant elision. Since the simplification here does not impinge upon the main argument below, I restrict my attention to place, disregarding the etymological reanalysis of the $/ \mathrm{k} / \approx / \mathrm{g} /$ alternation, for instance.

$$
\text { Singular Plural Gloss }
$$

$\begin{array}{lll}\text { hond } & \text { hon } & \text { dog } \\ \text { ftant } & \text { jtey } & \text { situation } \\ \text { grond } & \text { gron } & \text { ground } \\ \text { pond } & \text { pon } & \text { pound } \\ \text { cnd } & \text { en } & \text { end } \\ \text { faind } & \text { fain } & \text { enemy } \\ \text { viend } & \text { vien } & \text { wind } \\ \text { doek } & \text { doe } & \text { day } \\ \text { vek } & \text { ve } & \text { way } \\ \text { ftek } & \text { jt } \varepsilon & \text { path } \\ \text { gank } & \text { gej } & \text { walk } \\ \text { krog } & \text { kre } & \text { jug }\end{array}$

b.

$\begin{array}{lll}\text { ager } & \text { ager } & \text { field } \\ \text { joor } & \text { joor } & \text { year } \\ \text { keil } & \text { keil } & \text { wedge } \\ \text { bigil } & \text { bigil } & \text { pimple } \\ \text { agsdhelm } & \text { agsdhelm } & \text { axe handle } \\ \text { bam } & \text { bam } & \text { tree } \\ \text { dan } & \text { dan } & \text { fir } \\ \text { bun } & \text { bun } & \text { bean } \\ \text { breib } & \text { breib } & \text { letter } \\ \text { led } & \text { led } & \text { burial } \\ \text { kis } & \text { kis } & \text { rake } \\ \text { kees } & \text { kees } & \text { cheese } \\ \text { Joof } & \text { Joof } & \text { sheep } \\ \text { fe } & \text { fe } & \text { fish } \\ \text { reef } & \text { reef } & \text { tire }\end{array}$

Following Golston and Wiese (1995:150-151), I assume that a single feature is multiply associated with more than one segment if the relevant segments are adjacent and share the same feature specification. Thus, adjacent homorganic 
segments share one and the same place feature. Particularly important for our purposes is that a sequence of a vowel and a dorsal consonant share the same dorsal feature as well, given the assumption that vowels are specified for dorsal. This observation leads to the following more formal generalization: final consonant deletion takes place if its place specification is preserved in the output representation whereas no deletion is permitted if the application of the deletion process eliminates an underlying place specification. This can be captured by the following ranking: Max-Place » RM » Max-Seg Plural $_{\text {. }}$

As exemplified in (34) and (35), this ranking correctly produces the contrast between (33a) and (33b). In (34), the last two consonants share the same place of articulation (i.e., coronal), and deletion of the final segment does not obliterate any place feature. Under duress of RM, segmental deletion is required. By contrast, in (35), the final two segments disagree in their place specifications, so they carry dorsal and labial independently. Deletion of the final consonant entails deletion of the labial feature. As a consequence, the best form does not undergo consonantal deletion at the expense of a RM violation. This analysis is similar to Lardil nominatives in the sense that RM is not always satisfied.

\begin{tabular}{|ll||l|c|c|}
\hline & /hond/ $/$ Plural & Max-Place & RM & Max-Seg $_{\text {Plural }}$ \\
\hline \hline a. & hond & & $* !$ & \\
\hline b. $\quad$ hon & & & $*$ \\
\hline
\end{tabular}

\begin{tabular}{|ll||c|c|c|}
\hline & \multicolumn{1}{|c||}{$/$ bam/ Plural } & Max-Place & RM & Max-Seg \\
\hline \hline a. $\quad$ blural \\
\hline b. $\quad$ bam & ba & $* !$ & & \\
\hline
\end{tabular}


This analysis is quite different from Golston and Wiese's (1995). Their essential claim is that final consonant deletion is ultimately phonological rather than morphological. Since phonological alternations are explained by the Markedness » Faithfulness schema, their claim is tantamount to positing a markedness constraint as the driving force of consonant elision. Paying attention to the fact that plural forms created by final consonant deletion are always closed by a sonorant, they assume Son] $]_{\text {Plural }}$ which requires all plural forms to end in a sonorant (Golston and Wiese 1995:152). As many examples in (33b) show, this constraint is often violated when a base closed by an obstruent does not undergo deletion. For the data in (33), MaxPlace » Son $]_{\text {Plural }}$ » Max-Seg yields the correct output, as demonstrated in (36) and (37) (Golston and Wiese employ Max-[F] instead of Max-Place).

\begin{tabular}{|c|c|c|c|c|}
\hline & /hond/ Plural & Max-Place & Son $]_{\text {Plural }}$ & Max-Seg \\
\hline a. & hond & & $* !$ & \\
\hline b. & hon & & & $*$ \\
\hline
\end{tabular}

\begin{tabular}{|ll||c|c|c|}
\hline & $/ \mathrm{bam} /{ }_{\text {Plural }}$ & Max-Place & Son $]_{\text {Plural }}$ & Max-Seg \\
\hline \hline a. $\quad$ bam & & $*$ & \\
\hline b. & ba & $* !$ & & $*$ \\
\hline
\end{tabular}

This analysis is similar to the RM-based one in the sense that the featural maximization constraint prevents consonant deletion when the final two segments disagree in their place specifications whereas it occurs as long as Max-Place is satisfied. But the two analyses are fundamentally different. In my analysis, final consonant deletion is morphologically conditioned whereas it is phonologically 
derived according to Golston and Wiese. Given the morpheme-specific marking on the phonological markedness constraint, however, their analysis still depends on morphological information, and therefore, their central claim that the final consonant deletion is ultimately phonological cannot be well maintained. One conceivable objection to my RM-based analysis is that the fact that all forms with consonant deletion end in a sonorant would be accidental while this is an expected result of Son $]_{\text {Plural }}$-based account. This objection is not quite valid. Given the strong crosslinguistic tendency that the sonority value decreases toward the syllable peripheries (Clements 1990), the first (closer to the nucleus) member of a complex coda should be more sonorous than the second member. More concretely, a sequence of a sonorant and an obstruent is wellformed, but the reverse combination is not. Thus, the sonority principle prevents base forms closed by an obstruent after the following word-final consonant undergoes deletion. There is literally no exception to this in the examples in (33) as well as in more comprehensive Hessian German data collected in Golston and Wiese (1995). Hence, I conclude that the sonorant closure effect is simply an emergent property following from a more general consideration of syllable wellformedness in natural languages.

Golston and Wiese's (1995) analysis is vulnerable to a further criticism. As briefly stated in section 3.2.2, Itô and Mester (1999b) investigate what kind of constraints can be sensitive to particular morphological information in reduplication, arguing against morphologically governed markedness constraints. Horwood (1999) also discusses that the existence of morpheme-specific markedness constraints would permit $*$ Complex $]_{\text {Plural }}$, for example, but given the possibility of $*$ Complex $]_{\text {Plural }}$ » Dep, we would predict productive epenthesis to break up all consonant clusters to derive 
plural forms. This type of highly unlikely morphology is easily multiplied by positing a morpheme-particular markedness constraint outranking some relevant faithfulness constraint. Both Itô and Mester (1999b) and Horwood (1999) point out that the existence of markedness constraints operative only in a certain morphological domain gives rise to pathological empirical consequences. Given these arguments, the plausibility of Son $]_{\text {Plural }}$ becomes dubious.

I conjecture that the reason why morpheme-specific markedness constraints are implausible is closely related to the nature of nonconcatenative morphology that the application of some nonconcatenative stem change creates a more marked representation. A morpheme-specific markedness constraint needs to be ranked over competing faithfulness constraints to yield a visible effect, but this implies that a derived form is phonologically more harmonic than the input, contrary to fact. This suggests that the existence of morpheme-particular markedness constraints should be eliminated. In reverse, the non-existence of such markedness constraints explain why nonconcatenative morphology yields phonologically less harmonic structures.

Horwood's (1999) analysis is the same as mine in that final consonant deletion is considered as a morphologically conditioned process although his account relies on anti-faithfulness theory. His analysis is simply replacing Son $]_{\text {Plural }}$ with $\neg$ Max-C. I have already provided ample arguments against anti-faithfulness theory in general as well as against its application to subtractive morphology in particular. But it is worth repeating here that employing a consonant-particular $\neg$ Max is undesirable because the postulation of such a constraint entails the logical existence of those languages in which constituent-internal morphological subtraction is employed, as discussed in section 3.2.1. 


\subsubsection{Summary}

In sum, I have investigated concrete subtractive morphology data to implement the general proposal in chapter 2. I exemplified that subtractive morphology occurs as the phonological exponence of a morpheme if it does not contain any phonological substance and if RM outranks Max. This conforms to the general schema of nonconcatenative morphology developed in section 2.4. Besides this general property, I discussed several important issues as well. In section 3.2.1, the most significant lesson is that relativization of RM (instead of Faith) is not a valid alternative. I argued that this alternative schema results in a ranking paradox when some phonological markedness constraint is operative in the relevant word formation. Furthermore, RMT is grammar dependent, preserving a virtue of anti-faithfulness theory maintained by Alderete (1999) and Horwood (1999).

In section 3.2.2, I analyzed the nominative formation in Lardil, which poses an empirical problem to anti-faithfulness theory. Given that the output of a bare stem does not have to be an actually occurring form under RMT, Lardil nominatives are accommodated without any problem. The presented analysis further strengthens grammar dependency of RMT because morphological subtraction interacts with various concomitant phonological requirements within a single constraint hierarchy. One such effect is the blocking of morphological subtraction due to word minimality. This entails a violation of RM, but this is an expected result.

In section 3.2.3, I discussed the deverbal noun formation in Icelandic. What Benua (1995) takes as under-application is simply an effect of morpheme-specific faithfulness. Finally, in section 3.2.4, I examined the plural formation in Hessian German, a case alleged to be phonological by Golston and Wiese (1995). I argued 
that their claim is invalid, taking back the relevant data to the morphological side. Theoretically significant is the discussion that markedness constraints cannot be specific to a certain morpheme. This is closely tied to the nature of nonconcatenative morphology that forms derived through some stem change are phonologically less harmonic than the underlying representation.

\subsection{Templatic Truncation}

This section is devoted to templatic truncation. As mentioned at the outset of this chapter, templatic truncation is different in nature from subtractive morphology in two major formal respects. First, subtractive morphology is grammaticalized, and therefore, its absence results in a serious trouble in language communication. By contrast, templatic truncation is not associated with any morphosyntactic function, indicating that it is not an indispensable word formation in natural languages in the sense that no serious trouble would arise by its absence. Second, the deleted portion is constant in subtractive morphology, disregarding phonological factors, whereas the residue of deletion is prosodically constant in templatic truncation. The special focus of this section is on this second point, namely how these mirror-imaged behaviors of the two modes of truncation are accounted for in a principled manner.

In essence, my response to these two points is as follows. Concerning the grammaticality issue, I claim that there exist non-grammaticalized morphemes which exercise influence on morphology, which are called here sociolinguistic morphemes. Given RMT developed here, all morphological phenomena are driven by the presence of a morpheme. Language games, which are not grammaticalized in the linguistic systems either, also exhibit quite regular and productive phonological behaviors often 
consistent with various grammaticalized morphological operations (cf. Bagemihl 1989, Tateishi 1989; Itô, Kitagawa and Mester 1996, and Sanders 1999, 2000 among others), non-grammaticalized processes are governed by linguistic systems regulating grammaticalized phenomena. I thus claim that sociolinguistic morphemes play an important role in explaining templatic truncation with which no morphosyntactic changes are concerned. Second, as for the asymmetry regarding the second point (i.e., which is prosodically constant, the deleted portion or the residue?), I claim that this difference resides in the presence or absence of a set of size restrictor constraints yielding a templatic effect ranked over Max. I discuss below productive loanword abbreviations in contemporary Japanese to substantiate these two claims. Itô (1990) thoroughly examines the templatic truncation involved in loanword clipping in modern Japanese, and the following data and descriptive generalization largely owe to her work as well as Suzuki (1995). But the data transcriptions are approximately phonemic, mostly following the kunreishiki style of romanization. A moraic nasal is represented by $[\mathrm{N}]$.

A first set of data is given in (38a). All truncated forms are of bimoraic size. Many researchers have argued the importance of a bimoraic foot in various word formation processes in Japanese (Poser 1984a, 1990; Tateishi 1989; Itô 1990; Mester 1990; Itô and Mester 1992; Suzuki 1995; Itô, Kitagawa and Mester 1996), and the examples in (38a) conform to this generalization. Various other word formations like hypocoristics, rustic girls' names, geisha client names, renyookei reduplication, and mimetic reduplication are prosodically defined either as a single heavy syllable or as two light syllables. The examples in (38a) exhibit a quantitative trochee along with these word formations. The quantitative trochee is independently supported by the 
fact that accent falls on the initial syllable of the clipped base in these word formations, as shown in (38b) (Suzuki 1995:455). Crucially, a regular accentuation pattern emerges despite the fact that the base forms do not follow a uniform accentuation pattern.

a. Base form

\begin{tabular}{|c|c|}
\hline herikoputaa & heri \\
\hline amatyua & ama \\
\hline purofessyonaru & puro \\
\hline ruporutaaju & rupo \\
\hline tyokoreeto & tyoko \\
\hline terorizumu & tero \\
\hline birudiNgu & biru \\
\hline opereesyoN & ope \\
\hline sutoraiki & suto \\
\hline konekushoN & kone \\
\hline adoresu & ado \\
\hline raboratorii & rabo \\
\hline Base form & Hypocoristics form \\
\hline Kaori & Káo-tyaN \\
\hline Mínami & Mína-tyaN \\
\hline KeNiti & KéN-tyaN \\
\hline Dáisuke & Dái-tyaN \\
\hline
\end{tabular}

My analysis is that loanword truncation is motivated by a sociolinguistic (or non-grammaticalized) morpheme, called $\tau$ here, whatever sociolinguistic meaning it has. This morpheme does not contain any underlying phonological substance, and truncation takes place as a particular strategy to express the presence of morpheme $\tau$ on the surface. Thus, the basic schema operative here is $\mathrm{RM} » \mathrm{Max}_{\tau}$. This constraint ranking motivates truncation to satisfy RM with respect to morpheme $\tau$, but minimal deletion of the base is sufficient for the fulfillment of RM. My claim is that a set of constraints is ranked over $\operatorname{Max}_{\tau}$ to derive the templatic effect. In short, the template of bimoraic size is derived through the combination of the following three constraints: 
$\operatorname{FtBin}(\mu), \operatorname{Parse}(\sigma)$, and Align-L(Ft,PrWd). $\operatorname{FtBin}(\mu)$ is a specific version of a foot binarity constraint (McCarthy and Prince 1986 et seq.) restricting the binarity domain to morae (cf. Hewitt 1994). Parse $(\sigma)$ dictates that every syllable be parsed into a foot (Prince and Smolensky 1993:58). Given these two constraints, a prosodic word must consist strictly of bimoraic feet. Finally, Align-L(Ft,PrWd) demands the left edge alignment between a foot and a prosodic word. Since only a word-initial foot satisfies it, maximally one foot is permitted within a prosodic word. These three constraints together yield one bimoraic foot as the output. I call this set of constraints 'prosodic constraint' (ProsCon). As illustrated in (39), ProsCon outranks Max $_{\tau}$ so that as many segments as needed are deleted to achieve the output of bimoraic size at the expense of many $\operatorname{Max}_{\tau}$ violations.

\begin{tabular}{|ll||c|c|c|}
\hline & /herikoputaa/ & ProsCon & $\mathrm{RM}$ & $\mathrm{Max}_{\tau}$ \\
\hline \hline a. & (he.ri)(ko.pu)(taa) & $* !$ & $*$ & \\
\hline b. & (he.ri)(ko.pu) & $* !$ & & $* *$ \\
\hline c. & (he.ri)(ko) & $* !$ & & $* * * *$ \\
\hline d. & (he.ri)ko & $* !$ & & $* * * *$ \\
\hline e. $\quad$ (he.ri) & & & $* * * * * *$ \\
\hline
\end{tabular}

Importantly, the role of RM is made invisible due to ProsCon. Given RM » $\mathrm{Max}_{\tau}, \mathrm{RM}$ is satisfied by deleting at least one base segment, but ProsCon is usually satisfied by deleting more than one segment. This suggests that the violation incurred for the satisfaction of RM is a subset of the violation necessary to satisfy ProsCon. The strict ranking between ProsCon and RM is motivated by the fact that truncation is entirely blocked when the base is already minimal (e.g., [paN] 'bread' borrowed from 
Portuguese and [mesu] 'scalpel' borrowed from Dutch). Since RM is more specific than ProsCon, ProsCon » RM suggests the inactivity of RM due to Paninian Theorem (Prince and Smolensky 1993:81-82). I claim that this is what accounts for the crucial difference between subtractive morphology and templatic truncation: what is elided is constant in subtractive morphology while what remains is prosodically invariable in templatic truncation. This situation is akin to affixation like the plural morpheme in English. Consider affixation where the phonological element of a morpheme surfaces faithfully. The role of RM is normally masked in affixation, so the functions of RM and the relevant faithfulness constraints overlap.

However, templatic truncation still exhibits some similarities to subtractive morphology. First, RM is violated under certain circumstances. Besides those cases where the base is already of minimal size discussed above, templatic truncation is blocked when the base consists of a heavy syllable followed by a light one, as exemplified in (40).

$\begin{array}{lll}\text { Base form } & \text { Truncation form } & \text { Gloss } \\ \text { paNtu } & \text { N/A } & \text { pants } \\ \text { fuudo } & \text { N/A } & \text { food } \\ \text { syuuzu } & \text { N/A } & \text { shoes } \\ \text { puuru } & \text { N/A } & \text { pool }\end{array}$

Given the constraint ranking established in (39), this is rather surprising since deleting a final syllable satisfies both ProsCon and RM. Following Suzuki (1995) and Itô, Kitagawa and Mester (1996), I employ NonFinality as a pertinent constraint. This constraint prohibits the head from occupying the right periphery of a word (Prince and Smolensky 1993:40). The relevant prosodic category is the syllable. Since final syllable deletion results in a monosyllabic bimoraic foot, the head syllable 
is positioned at the right edge of the prosodic word, violating NonFinality. This is shown in (41). NonFinality does not play any role for the data in (38a) since the resultant foot consists of two light syllables, the first one being the head.

\begin{tabular}{|ll||c|c|c|c|}
\hline & $/ \mathrm{paNtu} / \tau$ & NonFinality & ProsCon & $\mathrm{RM}$ & $\operatorname{Max}_{\tau}$ \\
\hline \hline a. $\quad(\mathrm{paN}) \mathrm{tu}$ & & $*$ & $*$ & \\
\hline b. & $(\mathrm{paN})$ & $* !$ & & & $* *$ \\
\hline
\end{tabular}

The examples in (40) are not isolated cases where NonFinality plays an essential role. First, the examples in (42a) are the mirror image of (40): the first two syllables of the bases consist of a light and a heavy syllable. In such cases, the truncation form keeps up through the vowel of the second syllable from the beginning of the base, deleting a moraic nasal or the second half of a diphthong or shortening the long vowel of the second syllable. This observation is also explained by NonFinality: [(de.mo)] incurs more $\mathrm{Max}_{\tau}$ violations, but [de.(moN)] violates NonFinality which is never violated in loanword abbreviations in modern Japanese. Second, NonFinality is active when the base-initial two syllables are a sequence of a heavy and a light syllables, as illustrated in (42b). If all syllables were deleted except the first heavy one, the outcome is a monosyllabic heavy syllable. Comparing [(saN)] and $[(\mathrm{saN}) . \mathrm{do}]$, for example, the first form violates NonFinality. Thus, [(saN).do] is more harmonic than $[(\mathrm{saN})]$.

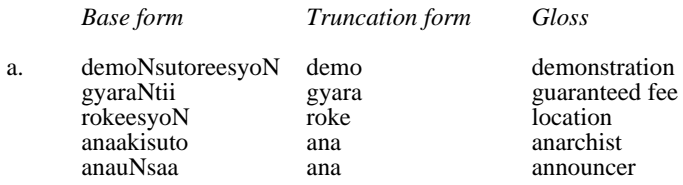


b.

$\begin{array}{lll}\text { saNdoitti } & \text { saNdo } & \text { sandwich } \\ \text { siNpasaizaa } & \text { siNpa } & \text { sympathizer } \\ \text { aNpurifaiaa } & \text { aNpu } & \text { amplifier } \\ \text { koNbineesyoN } & \text { koNbi } & \text { combination } \\ \text { paNkutyaa } & \text { paNku } & \text { puncture, a blowout } \\ \text { maikurohoN } & \text { maiku } & \text { microphone } \\ \text { saikederikku } & \text { saike } & \text { psychedelic } \\ \text { daiyamondo } & \text { daiya } & \text { diamond } \\ \text { paamaneNto } & \text { paama } & \text { permanent (hairstyle) }\end{array}$

In addition to the violability of RM in templatic truncation, the discussion above illuminates another similarity between subtractive morphology and templatic abbreviations, namely grammar dependency. The constraints that regulate templatic truncation are not just RM and Max. First, ProsCon plays a pivotal role in deriving the templatic effect. Second, NonFinality further regulates the restrictions imposed by ProsCon. A collection of constraints is needed to capture a templatic effect, but the interaction of NonFinality with other constraints is particularly a grammar dependent aspect since it requires deviance from strict templatism. Suzuki's (1995) argument that high ranked NonFinality is the driving force to obtain the loose interpretation of minimal words (Itô and Mester 1992) is essentially recaptured as an argument for grammar dependency of Japanese prosodic morphology.

The parallelism of subtractive morphology and templatic truncation can be clearly highlighted by comparing my analysis with Benua's (1995, 1997). As a representative example of templatic truncation, she considers hypocoristics in Japanese (Poser 1984a, 1990; Mester 1990). Besides hypocoristic forms with no modification of base names, productive hypocoristic truncation results in a bimoraic base followed by a special diminutive suffix -tyaN. Relevant data are provided in (43) (Benua 1995:117). Her explanation for the bimoraic templatic effect is the same 
as the one presented above (i.e., the combination of $\operatorname{FtBin}(\mu)$, $\operatorname{Parse}(\sigma)$, and AlignL(Ft,PrWd)). I keep calling this set of constraints ProsCon.

$\begin{array}{ll}\text { Base form } & \text { Hypocoristics form } \\ \text { Midori } & \text { Mido-tyaN, Mii-tyaN } \\ \text { Yooko } & \text { Yoko-tyaN, Yoo-tyaN } \\ \text { Akira } & \text { Aki-tyaN } \\ \text { Hiromi } & \text { Hiro-tyaN, Romi-tyaN } \\ \text { Mariko } & \text { Mari-tyaN, Mako-tyaN } \\ \text { JuNko } & \text { JuN-tyaN } \\ \text { Hanako } & \text { Hana-tyaN, Haa-tyaN, Hat-tyaN } \\ \text { Kazuhiko } & \text { Kazu-tyaN }\end{array}$

Since truncation takes place in the hypocoristic forms derived from existing (output) names, Benua claims that ProsCon outranks Max-BT(OO), as demonstrated in (44). On the other hand, the fact that no truncation occurs otherwise motivates Max-IO » ProsCon, as shown in (45). By transitivity, Max-IO » ProsCon » Max-OO follows.

\begin{tabular}{|ll||c|c|}
\hline & /Kazuhiko/ & ProsCon & Max-OO \\
\hline \hline a. & $($ Ka.zu $)($ hi.ko $)$ & $* !$ & \\
\hline b. & $($ Ka.zu $)$ & & $* * * *$ \\
\hline
\end{tabular}

\begin{tabular}{|ll||c|c|}
\hline & /Kazuhiko/ & Max-IO & ProsCon \\
\hline \hline a. & $($ Ka.zu $)($ hi.ko $)$ & & $*$ \\
\hline b. & $($ Ka.zu $)$ & $* ! * * *$ & \\
\hline
\end{tabular}

This analysis is analogous to mine, but raises a serious problem when it is considered in conjunction with her own analysis of Icelandic deverbal noun formation discussed in section 3.2.3. Recall from (31) and (32) that she argues for Max-OO » 
SonCon » Max-IO. This constraint ranking is exactly the mirror image of the ranking necessary to account for templatic truncation since Max-OO and Max-IO are ranked oppositely with respect to a phonological markedness constraint. But Max-OO » Max-IO and Max-IO » Max-OO are incompatible with each other, and therefore, her system cannot deal with languages which possess both subtractive morphology and templatic truncation. In effect, postulating these two ranking schemata is tantamount to claiming that subtractive morphology and templatic truncation are regulated by two distinct mechanisms. To circumvent this problem, Benua's analysis would need to incorporate morpheme sensitivity of faithfulness constraint, but this is precisely what is proposed and implemented in this work.

Under RMT, the only property that differentiates subtractive morphology and templatic truncation is whether prosodic constraints deriving a templatic effect are ranked over the relevant Max constraint. The two types of truncatory morphology are both morpheme-driven, and the morphemes of relevance are devoid of phonological content. Truncation takes place under the $\mathrm{RM} » \mathrm{Max}_{\alpha}$ ranking, where $\alpha$ stands for an appropriate morpheme. Again, this is the elementary mechanism common to the two truncation modes, suggesting that the kind of ranking paradox encountered by Benua $(1995,1997)$ does not arise.

Summarizing this section, I argued that templatic truncation is basically explained in the same manner as subtractive morphology. Given that templatic truncation is not a phonologically conditioned phenomenon, I claimed that it is motivated by some non-grammaticalized morpheme necessary for sociolinguistic reasons. Since such morphemes do not contain any underlying phonological element, the base form is subject to some phonological modification to satisfy RM. In 
Japanese, loanwords are productively shortened, so Max associated with the relevant sociolinguistic morpheme is ranked beneath RM. But the role of RM is made opaque because of the presence of a set of prosodic constraints. This is a natural outcome since the prosodic constraints function as a size restrictor, covering the role played by RM. Nevertheless, templatic truncation shares some properties with subtractive morphology or nonconcatenative morphology in general. First, RM is violated under certain circumstances. This suggests that RM is nothing special in templatic morphology. Second, templatic truncation is still governed by the influence of other constraints. In this sense, it is also explained by the grammar dependent system.

\subsection{Summary}

I discussed two types of truncatory morphology in this chapter: subtractive morphology and templatic truncation. They must be distinguished in two formal respects: whether they are grammaticalized or not, and what is prosodically constant, the deleted part or the residue. First, these two types of truncation are both morpheme-driven. Subtractive morphology is associated with grammaticalized morphemes, but templatic truncation with non-grammaticalized ones. Second, RM » Max is the common schema underlying subtractive morphology and templatic truncation. Although the function of RM is obscured by a set of prosodic constraints regulating the prosodic size of the output in templatic truncation, this is what distinguishes the two kinds of truncation since such prosodic constraints are not relevant in subtractive morphology. I pointed out that they still exhibit some similar properties: violability of RM and grammar dependency of the entire system. These properties are expected given the basic architecture of OT that all constraints are 
potentially violable and that the ultimate output is determined by their interactions. An interesting question remains: why do non-grammaticalized morphemes exhibit templatic effects while grammaticalized ones do not? I leave this issue open for future research. 


\section{Chapter 4}

\section{Optimality of Nonconcatenative Allomorphs}

\subsection{Introduction}

In this chapter, I explore multiple nonconcatenative allomorphs. Morphemes often have several different surface realizational forms (i.e., allomorphs). For example, the English plural suffix receives three different realizations depending on the phonological context: [s] after a voiceless non-strident segment, [z] after a voiced non-strident segment, and [az] after a strident. Given various possibilities of expressing a morpheme by base modification, however, it is naturally expected that similar situations are observed in nonconcatenative morphology. More concretely, we expect that various nonconcatenative operations should be able to be employed to denote a particular morphosyntactic function depending on the phonological shape of the given base, as schematically depicted in (1), where $\alpha, \beta$, and $\gamma$ represent output forms created by performing some base modification rather than by adding an affix to realize morpheme $\mu$.

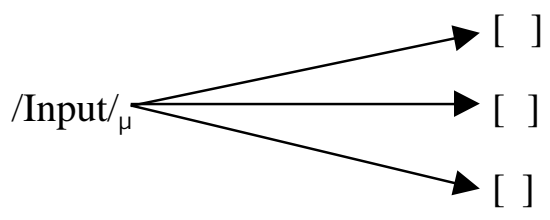

This state of affairs is actually attested in natural languages, particularly in the Salishan language family. In (1), the available phonological changes of a stem are mutually exclusive since they are all exponents of the same morpheme. This is a rather trivial fact which follows from the definition of allomorphs. But what makes nonconcatenative allomorphs interesting is the fact that the choice of a particular 
nonconcatenative allomorph is global. The selection of an allomorph in the English plural formation is quite parochial in the sense that the relevant phonological context is the immediately preceding segment. In nonconcatenative allomorphs investigated here, much more extensive information of the base must be taken into consideration.

It is true that nonconcatenative morphemic alternations are crosslinguistically rare, but the amount of attention paid to those cases has been scarce in the earlier literature. The goal of this chapter is thus to examine some examples and propose a unified account for them within the theoretical context developed in chapter 2 . Particularly, I focus on the following two questions: (i) what precisely is the driving force of such nonconcatenative morphemic alternations?, and (ii) how should the driving force be successfully reflected in a formal explanation?

The rest of this chapter is mapped out as follows. In section 4.2, I provide an overall answer to the two questions addressed above. I argue that the reason why a variety of base deformations occur resides in natural languages' desire to achieve phonologically less marked output forms, hence optimality of nonconcatenative allomorphs. This optimization is accomplished through interactions of phonological markedness constraints and various faithfulness constraints, the latter being specific to a given morphosyntactic category. The general proposal outlined in section 4.2 is subsequently tested against actual data. Section 4.3 discusses the continuative formation in Upriver Halkomelem, a Salishan language. In this word formation, four varieties of allomorphs come into the picture: reduplication, $h-$-prefixation, vowel lengthening, and stress shift. Section 4.4 is devoted to a case study of another Salishan language, Saanich. In this language, the actual aspect morpheme is phonologically realized by metathesis, reduplication, or -infixation. In the course of 
investigating these cases, I critically review some derivational and constraint-based approaches taken in the earlier literature and argue that they are not satisfactory. To this end, I illuminate the significant importance of the role played by RM. In section 4.5, I investigate the incomplete phase formation in Rotuman, a language genetically unrelated to the Salishan languages. The two factors which differentiate this case from the two Salishan language examples are first that the relevant word formation is strictly templatic, and second that, occasionally under duress of undominated constraints, no phonological exponent appears. Finally, section 4.6 summarizes the main results of this chapter.

\subsection{Nonconcatenative Allomorphs as Word Optimization}

This section outlines the specific issue to be addressed and provides a general proposal to deal with it in a principled way. As discussed and exemplified in chapters 2 and 3, the core constraint ranking to explain various nonconcatenative operations is $\mathrm{RM} \gg$ Faith, the specific Faith determining the particular phonological modification that a base undergoes. Given this basis, simply ranking multiple faithfulness constraints beneath RM is not sufficient to derive various context-sensitive operations, although ranking all relevant Faith-IO below RM is a minimal prerequisite. Suppose that both Max-IO-Seg and Linearity-IO are outranked by RM. But this is not a sufficient condition to derive the effect of multiple nonconcatenative allomorphs. Given the spirit of strict dominance holding among constraints, Max » Linearity or Linearity » Max should hold. In the former ranking, metathesis is more harmonic while it is suboptimal given the latter ranking, modulo everything else being equal. This indicates that any ranking permutation of RM and faithfulness 
constraints yields at best only one type of nonconcatenative allomorph, the one which violates the lowest ranked faithfulness constraint.

The role of markedness constraints becomes crucial at this point. They are often in conflict with faithfulness constraints, and their interactions play a key role in capturing the distribution of multiple nonconcatenative allomorphs. The gist of the idea is that a violation of a high ranked faithfulness constraint is forced when the violation of a lower ranked constraint leads to a violation of some markedness constraint outranking the higher ranked faithfulness constraint. Otherwise, violating the lower ranked faithfulness constraint is less costly.

To illustrate this idea, consider the following schematic example. In language $\mathrm{L}$, the morphosyntactic category $\alpha$ is phonologically realized either by metathesis or by subtractive morphology. This language is free from any syllable structure restrictions except the inviolable onset requirement. Metathesis is the elsewhere case, so $\operatorname{Max}_{\alpha}$ outranks Linearity ${ }_{\alpha}$. Given this set-up, consider the following two input forms: /CVC/ and /CVCV/. Assuming Onset and RM as undominated constraints, various candidates are evaluated for these two inputs. First, as illustrated in (2), morpheme $\alpha$ is phonologically denoted by metathesis when /CVC/ is the input. Since neither metathesis nor subtractive morphology incurs a violation of Onset, violating low ranked Linearity is the most harmonic. By contrast, metathesis is suboptimal when the input is /CVCV/ because Onset is violated as the result of metathesis. An important observation is that the effect of Onset enters the picture only when metathesis creates an onsetless syllable, and this is the only context where subtractive morphology is chosen as the optimal nonconcatenative allomorph. This accords with the basic tenet of OT that constraints are violable only when it is necessary for the 
satisfaction of higher constraints (Prince and Smolensky 1993:chapter 3). To take advantage of multiple nonconcatenative allomorphs is therefore an effort of languages to optimize the overall word form by avoiding phonologically marked structures or representations.

\begin{tabular}{|ll||l|l|l|l|}
\hline & $/ \mathrm{CVC}_{\alpha}$ & Onset & $\mathrm{RM}$ & $\operatorname{Max}_{\alpha}$ & $\operatorname{Lin}_{\alpha}$ \\
\hline \hline a. & $\mathrm{CVC}$ & & $* !$ & & \\
\hline b. & $\mathrm{CV}$ & & & $* !$ & \\
\hline c. & & & & & $*$ \\
\hline
\end{tabular}

\begin{tabular}{|c|c|c|c|c|c|c|}
\hline & & $/ \mathrm{CVCV} /_{\alpha}$ & Onset & RM & $\operatorname{Max}_{\alpha}$ & $\operatorname{Lin}_{\alpha}$ \\
\hline a & & CV.CV & & $* !$ & & \\
\hline & 吗 & $\mathrm{CVC}$ & & & * & \\
\hline $\mathrm{c}$ & & CV.VC & $* !$ & & & $*$ \\
\hline
\end{tabular}

The most significant merit of this constraint-based analysis lies in its explanatory level and strong predictability. First, it is explanatorily satisfactory because the reason why both metathesis and subtractive morphology enter the picture is quite clear. Onsetless syllables are entirely prohibited in L, and the reason why the otherwise cheapest strategy is occasionally blocked directly follows from the normal phonology of L. Second, the strong predictive power is hard-wired into the theoretical architecture. Since various morphemic alternations are explained by a single constraint ranking, all relevant generalizations are packed in the same box. The distribution of various nonconcatenative allomorphs follows from the single ranking, so other cases are predictable. This is a desirable consequence from the 
perspective of language acquisition since the language learner does not need to be exposed to all individual examples.

The reason why languages exhibit nonconcatenative allomorphs is attributed to the general demand of natural languages to achieve output forms that are as unmarked as possible. This intuition is directly captured by constraint-based OT. Allomorphemic optimization is nothing special to nonconcatenative morphology. A simple example is drawn from the plural suffix in English. The fact that the morpheme surfaces in three different varieties is considered as the effect of markedness constraints requiring voicing assimilation and prohibiting adjacent stridents. This can be seen as an effect motivated by the relevant markedness constraints overriding the relevant faithfulness constraints. Thus, the principle underlying allomorphemic optimization is a general one.

Finally, I discuss a formal aspect of the system deriving nonconcatenative allomorphemic alternations: interactions between markedness and faithfulness constraints. More specifically, I address when a markedness constraint meaningfully interacts with faithfulness constraints. Again, the faithfulness constraints ranked lower than RM are constraints whose violations are allowed for the satisfaction of RM. Given this background, suppose that three faithfulness constraints are outranked by $R M$, as in (4). $M_{a}-M_{d}$ represent slots that a markedness constraint can potentially occupy. A preliminary consideration eliminates uninteresting cases. With respect to $\mathrm{M}_{\mathrm{d}}$, all faithfulness constraints outrank the markedness constraint, so it has no impact on the relevant morphosyntactic formation: it is always violated. In the same way, $\mathbf{M}_{\mathrm{c}}$ does not have any surface effect either. Even if the violation of Faith-D entails a violation of the markedness constraint, violating both $\mathrm{M}_{\mathrm{c}}$ and Faith-D is still more 
harmonic than violating only either Faith-B or Faith-C. This suggests that languages do not present any explicit empirical evidence for the distinction between $M_{c}$ and $M_{d}$.

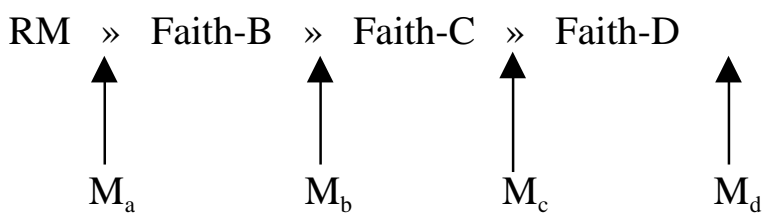

Focusing on $M_{a}$ and $M_{b}$, consider the tableaux in (5) and (6). $M_{b}$ is active only when the violation of Faith-D results in a violation of $M_{b}\left(\right.$ and/or $\left.M_{a}\right)$ as well. Despite the fact that Faith-C is ranked over Faith-D, candidate (5b) is the optimal in this case provided that it satisfies $\mathrm{M}_{\mathrm{b}}$. Interactions of $\mathrm{M}_{\mathrm{b}}$ with Faith-B have no effect on the selection of the winner. $\mathrm{M}_{\mathrm{a}}$ is the unique constraint that gives candidate [AB'CD] the chance to be the winning form. It is crucial that $\mathrm{M}_{\mathrm{a}}$ conflict with both Faith-C and Faith-D since (6a) or (6b) would surface otherwise.

\begin{tabular}{|ll||c|c|c|c|c|}
\hline & \multicolumn{1}{|c|}{$/ \mathrm{ABCD}_{\alpha}$} & $\mathrm{M}_{\mathrm{a}}$ & Faith-B & $\mathrm{M}_{\mathrm{b}}$ & Faith-C & Faith-D \\
\hline \hline a. & $\mathrm{ABCD}$ & & & $* !$ & & $*$ \\
\hline b. & $\mathrm{ABC} \mathrm{D}$ & & & & $*$ & \\
\hline c. & $\mathrm{AB} \mathrm{C}^{\prime} \mathrm{CD}$ & & $* !$ & & & \\
\hline
\end{tabular}

(6)

\begin{tabular}{|ll||c|c|c|c|c|}
\hline & \multicolumn{1}{|c|}{} & & & \\
& $\mathrm{ABCD} / \alpha$ & $\mathrm{M}_{\mathrm{a}}$ & Faith-B & $\mathrm{M}_{\mathrm{b}}$ & Faith-C & Faith-D \\
\hline a. & $\mathrm{ABCD}$ & $* !$ & & & & $*$ \\
\hline b. & $\mathrm{ABC}{ }^{\prime} \mathrm{D}$ & $* !$ & & & $*$ & \\
\hline c. & $\mathrm{AB} \mathrm{C}^{\prime} \mathrm{CD}$ & & $*$ & & & \\
\hline
\end{tabular}

This consideration highlights the strict dominance of constraints maintained in OT. In essence, a markedness constraint plays an active role only when it blocks all less marked nonconcatenative allomorphs. In derivational terms, the process which 
violates the lowest ranked faithfulness constraint is the elsewhere case, and the process which incurs a violation of the highest ranked faithfulness constraint has the most severely restricted distribution.

\subsection{The Continuative in Upriver Halkomelem}

Upriver Halkomelem, a central coast Salishan language, exhibits a complicated pattern of nonconcatenative allomorphy in the continuative formation. The continuative implies that the action denoted by a root continues for a moderate length of time after onset (if a time of onset is implied or stated within the speech event) or before conclusion (if a time of conclusion is implied or stated within the speech event). In the absence of an implication or statement of onset or conclusion, the continuative action is assumed to be continuing indefinitely (Galloway 1993:261). The continuative aspect is opposed to the mutually exclusive and the more unmarked noncontinuative, and is realized in four different ways depending on the context: reduplication, $h$-prefixation, vowel lengthening, and stress shift, as schematized in (7). As discussed by Urbanczyk (1998), the two prefixal segments are epenthetic.

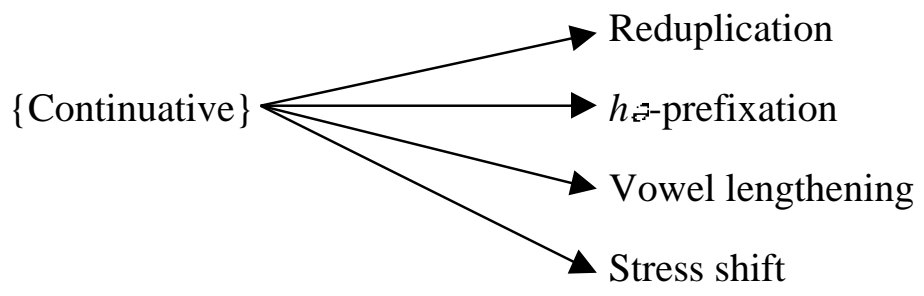

In this section, I demonstrate the proposal in the previous section with the continuative formation in Upriver Halkomelem. Relevant data are described by Galloway (1980, 1993) and analyzed by Urbanczyk (1998) in OT. However, her analysis is remarkably different from mine, so this example provides a good 
representative case to highlight my proposal. In section 4.3.1, I offer the basic facts. Section 4.3.2 is devoted my analysis. I also argue that nonconcatenative allomorphs present a serious empirical challenge to anti-faithfulness theory. Finally, section 4.3.3 provides a critical review of Urbanczyk's (1998) OT analysis.

\subsubsection{Facts and Descriptive Generalization}

Relevant examples excerpted from Galloway $(1980,1993)$ are provided in $(8)$, where stressed syllables are underlined.

a. Reduplication

\begin{tabular}{|c|c|c|c|}
\hline Noncontinuative & Gloss & Continuative & Gloss \\
\hline t'i.ləm & sing & t'i.ta.lam & singing \\
\hline wi.qes & yawn & wi.wว.qəs & yawning \\
\hline yiq & fall (of snow) & yi.yəq & falling (of snow) \\
\hline$x^{\mathrm{w}} \mathrm{a} \cdot y a m$ & sell & $x^{w} a \cdot x^{w} a \cdot y a m$ & selling \\
\hline t'i.com & swim & t'i.to.cəm & swimming \\
\hline
\end{tabular}

b. $\quad h$-prefixation

\begin{tabular}{|c|c|c|c|}
\hline Noncontinuative & Gloss & Continuative & $\begin{array}{l}\text { Gloss } \\
\text { swallowing }\end{array}$ \\
\hline$\underline{\text { ma.qat }}$ & swallow & $\underline{\text { ham. }} \cdot q^{\prime}$ at & $\begin{array}{l}\text { swallowing } \\
\text { drowning }\end{array}$ \\
\hline$\underline{w_{a} q^{\prime w}}$ & drown & $\underline{\text { haw }} q^{\prime \mathrm{w}}$ & drowning \\
\hline$\underline{\mathrm{maq}^{\prime}}$ & get full & hamq' & getting full \\
\hline
\end{tabular}

c. Vowel lengthening

\begin{tabular}{|c|c|c|c|}
\hline Noncontinuative & Gloss & Continuative & Gloss \\
\hline ?1. $\max$ & walk & ?ii.max & walking \\
\hline he.wo & hunt & 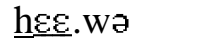 & hunting \\
\hline ha.qwat & smell & haa.qwot & smelling \\
\hline 2al. & groan & 2aal. & groaning \\
\hline ii.tat & sleep & 2ii.tat & sleeping \\
\hline
\end{tabular}

d. Stress shift

\begin{tabular}{|c|c|c|c|}
\hline Noncontinuative & Gloss & Continuative & Gloss \\
\hline sa.q $q^{\prime} \mathrm{ct}$ & split & $\underline{\text { sa }} \cdot q^{\prime} \varepsilon t$ & splitting \\
\hline X'a.wals & bark & $\overline{\chi^{\prime}}$ a. wals & barking \\
\hline caa. $\frac{10}{w} \cdot x^{\mathrm{w}}$ am & bleed & caa.lə. $x^{\mathrm{w}}$ əm & bleeding \\
\hline 4ə. $\mathrm{x}^{\mathrm{w}} \partial 4 . c \varepsilon$ & spit & $\underline{ \pm 0} \cdot \mathrm{x}^{\mathrm{w}} \partial 4 . \mathrm{c} \varepsilon$ & spitting \\
\hline
\end{tabular}


Relevant descriptive generalization is divided into two portions, phonological and allomorphemic. Starting with phonological generalization, we need to capture some properties. First, in reduplication, the reduplicant is constantly the initial CV of the base. Furthermore, the initial vowel of the base is reduced to a schwa. Second, in prefixation, a schwa is prefixed and the obligatory onset position is filled by another epenthetic segment $[\mathrm{h}]$. The first vowel of the noncontinuative base is subject to deletion. Urbanczyk (1998) provides evidence that $[\mathrm{h}]$ is epenthetic. Galloway (1993) discusses that resultative stems are obtained by adding the $s$ - stative prefix to intransitive continuative forms. As /ləcət/ $\rightarrow$ [səlcət] 'fill it/filed' indicates, [h] does not appear in this case. This makes sense if $[\mathrm{h}]$ is an epenthetic place holder when underlying segments are insufficient for the satisfaction of the onset requirement. In the resultative, the prefix $s$ - is available, so no epenthesis is needed (see Hukari 1978 for the same observation in Cowichan). Furthermore, Galloway (1993:118) remarks that [?] or [h] serves as an epenthetic segment to break up a vowel cluster to prohibit merger of two vowels. Finally, there is nothing special to say on vowel lengthening and stress shift. These generalizations are regarded as emergence of the unmarked (cf. Urbanczyk 1998).

Turning to the distribution of the four nonconcatenative allomorphs, the generalization is as follows. First, reduplication occurs when the noncontinuative base begins with $\mathrm{CV}(\mathrm{C} \neq$ laryngeal, and $\mathrm{V} \neq \mathrm{a})$, as shown in (8a). Second, prefixation takes place when the base-initial segments are a sequence of a sonorant and a stressed schwa (8b). Third, vowel lengthening is required if the base begins with a laryngeal consonant (8c), and finally, stress is shifted when the base carries stress on a noninitial syllable of the word (8d). The four processes are in complementary 
distribution, confirming that they belong to one and the same morpheme. Cases where a base begins with a sequence of an obstruent and a schwa are not considered since they do not conform to a uniform pattern, but this does not impinge on crucial points here. They are not discussed by Urbanczyk (1998) either.

\subsubsection{Analysis}

In this section, I develop an OT analysis of the distribution of the four nonconcatenative allomorphs involved in the continuative formation in Upriver Halkomelem. Noncontinuative forms are identical to bare stems, and therefore, it does not make any difference whether stems or noncontinuative forms are treated as bases of continuative forms, although bare stems are likely to be bases under the system developed in section 2.3. Given that the two prefixal segments are both epenthetic and that no other allomorphs depend on affixation, the continuative morpheme is entirely contentless. The distribution of the relevant allomorphs is resummarized in (9).

(9) Reduplication: When the base begins with CV ( $\mathrm{C} \neq$ laryngeal, and $\mathrm{V} \neq 2)$

$h$-prefixation: When the base-initial segments are a sequence of a sonorant and a schwa.

Vowel lengthening: When the base begins with a laryngeal consonant. Stress shift: When the base-initial syllable is stressless.

As discussed in chapter 2, reduplication and epenthesis violate Integrity and Dep respectively. As the constraint violated by vowel lengthening, I employ IdentLength which requires that the output length of segments be the same as that of their input correspondents. Furthermore, I assume Align-L(Heado,PrWd) as the driving force of stress shift. A relevant prosodic faithfulness constraint is violated when stress shift occurs, but it is abstracted away from in the following tableaux. Another 
essential ingredient is $*$ Stress[ə] militating against a stressed schwa. RM plays an essential role in addition to these constraints.

Beginning with reduplication, a tableau is provided in (10). (10a) violates RM because the continuative morpheme does not receive any phonological realization in the surface representation. RM is satisfied by all other candidates since their phonological deviance from the input can be potentially associated with the continuative morpheme.

\begin{tabular}{|c|c|c|c|c|c|c|c|}
\hline & /wi.qas/Cont. & Align & $\mathrm{RM}$ & $\begin{array}{l}\text { Ident- } \\
\text { Length }\end{array}$ & *Stress[a] & Integ & Dep \\
\hline a. & wi.qes & & $* !$ & & & & \\
\hline b. & wi-wo.qas & & & & & $* *$ & \\
\hline c. & haw.qəs & & & & $* !$ & & $* *$ \\
\hline d. & wii.qas & & & $* !$ & & & \\
\hline e. & 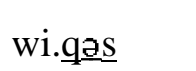 & *! & & & $*$ & & \\
\hline
\end{tabular}

(11) delineates a case of epenthetic prefixation. The tableau shows that candidates (11a), (11d), and (11e) lose the competition in the same way as in (10). In the evaluation of the relative wellformedness between (11b) and (11c), the crucial observation is that they both violate *Stress[a] because the first vowel of the input is a schwa. The decision is passed on to the lower ranked constraints. Given Integrity » Dep, reduplication is suboptimal. Comparing (10) and (11), the general proposal to explain nonconcatenative allomorphs is corroborated. In (11), *Stress[ə] plays no role in eliminating candidates, but in (10), it does. Given the constraint ranking, $h-$ prefixation is the most harmonic modulo no other factor comes into play. This maximal faithfulness is avoided when the violation of the lowest ranked faithfulness 
constraint is accompanied by a violation of a high ranked markedness constraint, as in (10).

\begin{tabular}{|c|c|c|c|c|c|c|c|}
\hline & $/ \underline{\mathrm{ma}} \cdot \mathrm{qat} / \mathrm{Cont}$ & Align & $\mathrm{RM}$ & $\begin{array}{l}\text { Ident- } \\
\text { Length }\end{array}$ & $*$ Stress[a] & Integ & Dep \\
\hline a. & $\underline{\text { me.qat }}$ & & $* !$ & & $*$ & & \\
\hline b. & ma.ma.qat & & & & $*$ & $* ! *$ & \\
\hline c. 喓 & həm.qat & & & & $*$ & & $* *$ \\
\hline d. & $\underline{\text { mag.qat }}$ & & & $* !$ & $*$ & & \\
\hline e. & ma.qat & $* !$ & & & $*$ & & \\
\hline
\end{tabular}

The stress shift examples in (8d) are rather trivial given the ranking demonstrated in (10) and (11). As demonstrated in (12), undominated Align$\mathrm{L}($ Head $\sigma$, PrWd $)$ selects the stress shift candidate as the optimal when the noncontinuative base carries stress non-initially. Two remarks are in order. First, the

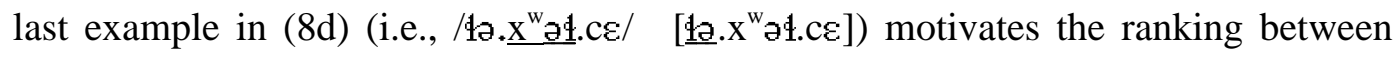
Align-L(Head $\sigma, \operatorname{PrWd})$ and $*$ Stress[ə]. Given that the first vowel is a schwa and the third vowel is a full vowel and that stress shifts to the word-initial syllable, the alignment constraint should outrank *Stress[ə]. Second, a prosodic faithfulness constraint which prohibits stress shift must be ranked below Align-L(Heado,PrWd). The fact that the alignment constraint is not obeyed by noncontinuative forms suggests that their stress assignment is not predictable. It is rather straightforwardly accounted for by postulating lexical stress in noncontinuative forms. The prosodic contrast between the noncontinuative and the continuative is captured by the

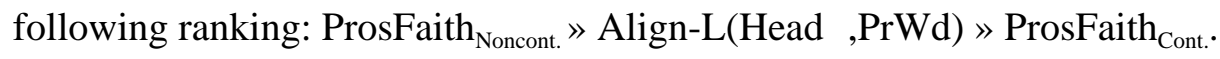




\begin{tabular}{|c|c|c|c|c|c|c|c|}
\hline & /4عl.q1i/Cont. & Align & $\mathrm{RM}$ & $\begin{array}{l}\text { Ident- } \\
\text { Length }\end{array}$ & *Stress[ə] & Integ & Dep \\
\hline a. & 4عl.qi & $* !$ & $*$ & & & & \\
\hline b. & 4c.lo.qi & $* !$ & & & & $* *$ & \\
\hline c. & hə.tel.qi & $* !$ & & & & & $* *$ \\
\hline d. & 4عel.qui & $* !$ & & $*$ & & & \\
\hline & $\underline{4 \varepsilon 1 . q i}$ & & & & & & \\
\hline
\end{tabular}

The analysis developed above is not sufficient for explicating the vowel lengthening cases in (8c). As it stands, the analysis predicts that reduplication is the best allomorph for those cases, contrary to fact. Investigating over two thousand words of Upriver Halkomelem, Urbanczyk (1998) reports that only twenty syllables were found which consist of a sequence of a glottal consonant and a schwa. This is significantly few, and she proposes a constraint which prohibits placeless syllables, where placeless syllables are defined as syllables whose member segments are all placeless. Capitalizing on her insight, I assume *Placeless $\sigma$. Placeless segments are laryngeal consonants and a schwa (see Bessell and Czaykowska-Higgins 1991 for arguments that laryngeal is placeless in Salishan languages in general).

Granting the undominated status to this constraint, consider (13). The crucial observation is that both (13b) (the reduplication allomorph) and (13c) (the $h z-$ prefixation allomorph) violate *Placeless $\sigma$. As mentioned in section 4.3.1, the baseinitial vowel is reduced to a schwa when reduplication occurs. This suggests that *Placeless $\sigma$ is violated when the noncontinuative base begins with a laryngeal consonant, exactly the context in which vowel lengthening is chosen as the best allomorphs. The $h ə$-prefixation candidate also violates *Placeless $\sigma$. As stated in 
section 4.3.1, the initial vowel of the noncontinuative base is subject to elision. Given that the prefixal segments are both placeless, $h$-prefixation necessarily leads to a violation of *Placeless $\sigma$ in the context where vowel lengthening takes place. Since Align-L(Head $\sigma, \operatorname{PrWd})$ and RM are undominated, vowel lengthening is the best allomorph although it violates Ident-Length.

\begin{tabular}{|c|c|c|c|c|c|c|c|}
\hline & /21i.max/Cont. & $*$ Pless $\sigma$ & Align & $\mathrm{RM}$ & $\begin{array}{l}\text { Ident- } \\
\text { Length }\end{array}$ & *Stress[ə] & Integ \\
\hline a. & 2i. $\max$ & & & $* !$ & & & \\
\hline $\mathrm{b}$. & $\underline{21} \cdot 2 a \cdot \max$ & $* !$ & & & & & $* *$ \\
\hline c. & $\underline{\text { hə2.max }}$ & $* !$ & & & & $*$ & \\
\hline d. 吗 & 2ii. $\max$ & & & & * & & \\
\hline e. & Ti.max & & $* !$ & & & $*$ & \\
\hline
\end{tabular}

Summarizing the analysis, the entire constraint hierarchy is given in (14). This constraint ranking highlights the most important idea in this chapter: multiple nonconcatenative allomorphs are a consequence of the existence of several faithfulness constraints ranked below RM and their interactions with markedness constraints. The allomorph which incurs only the lowest ranked faithfulness constraint violation is the least marked (i.e., $h$-prefixation), but the least costly option becomes more marked than other allomorphemic realizations when a high ranked markedness constraint is violated. As far as the pertinent faithfulness constraints are concerned, Dep Cont $_{\text {is ranked the lowest while Ident-Length }}$ Cont. is the highest ranked. This suggests that $h$-prefixation and vowel lengthening are the least and the most costly strategies to manifest the noncontinuative morpheme in the surface representation. Integrity ${ }_{\text {Cont. }}$ is ranked in-between, and therefore, reduplication 
has the intermediate markedness value. The overall evaluation of the optimal allomorph involves a large-scale computation compared with concatenative allomorphy because optimization of a whole word needs to be taken into account.

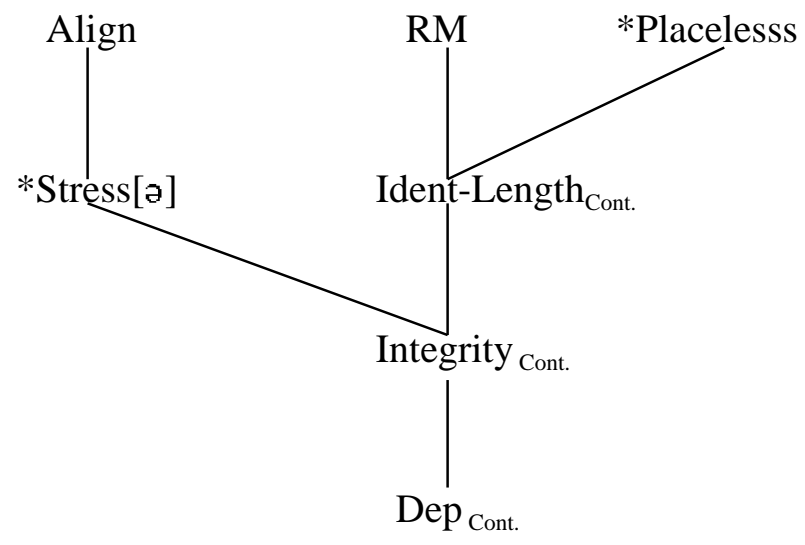

Allomorphemic alternations in nonconcatenative morphology as attested in Upriver Halkomelem present a serious empirical challenge to anti-faithfulness theory. Under this theory, a morpheme without any phonological substance should be associated with multiple anti-faithfulness constraints and that they are all ranked over the faithfulness counterparts. Thus, for the schematic example discussed in (2) and (3), ᄀMax and ᄀLinearity outrank Max and Linearity respectively. What is problematic here is that both morphological subtraction and metathesis are required irrespective of the presence or absence of markedness constraints, indicating that their distributional complementarity cannot be captured successfully. This problem arises because anti-faithfulness constraints and the corresponding faithfulness constraints are inherently incompatible with each other regardless of the context. As long as an anti-faithfulness constraint dominates the faithfulness counterpart, an anti-faithfulness effect surfaces on the output representation. 
The important fact is that even the introduction of markedness constraints does not rescue the undesirable situation. Suppose that $/ A B /$ is given in the underlying representation, and that $\neg$ Faith-A and $\neg$ Faith-B outrank Faith-A and FaithB respectively. Given that $\neg$ Faith-A and $\neg$ Faith-B require a faithfulness violation with respect to $/ \mathrm{A} /$ and $/ \mathrm{B} /$ respectively (i.e., $/ \mathrm{A} / \rightarrow\left[\mathrm{A}^{\prime}\right]$ and $/ \mathrm{B} / \rightarrow\left[\mathrm{B}^{\prime}\right]$ ), the relevant markedness constraints should be ${ }^{*} \mathrm{~A}^{\prime}$ and ${ }^{*} \mathrm{~B}$ '. For these markedness constraints to play an active role, it is essential that they be ranked over anti-faithfulness constraints because otherwise anti-faithfulness constraints are always dominant and therefore the markedness constraints play no deciding role. Given this set-up, however, antifaithfulness theory has potential to yield undesirable outcomes. First, there is no guarantee that the two markedness constraints are in conflict with the two antifaithfulness constraints. In such a case, no phonological realization of a morpheme appears on the surface. Second, nothing in anti-faithfulness theory prevents the possibility that the two markedness constraints and the two anti-faithfulness constraints are not in conflict with each other, producing multiple nonconcatenative stem modifications (e.g., subtractive morphology and umlaut) as the phonological exponents of one and the same morpheme. This argument points to the failure of anti-faithfulness theory to capture the complementary distribution of various nonconcatenative allomorphs. Given that those allomorphs do not surface in overlapping environments, what is called here multiple nonconcatenative allomorphs presents an empirical difficulty to anti-faithfulness theory.

By contrast, the RM-based approach does not run into the same problem. RM is a morphological faithfulness constraint which is not inherently antagonistic to phonological faithfulness constraints when a given morpheme does not possess any 
phonological material. The important implication is that only the violation of one faithfulness constraint is needed to satisfy high ranked RM since additional faithfulness violations are simply gratuitous: multiple nonconcatenative stem changes are always harmonically bounded in the sense of Prince and Smolensky (1993:176178). Which faithfulness violation must be incurred depends upon the overall wellformedness of the candidates yielded by Gen. The significant importance of the same faithfulness violation differs from context to context depending upon the given phonological base since various markedness constraints enter the picture in the wellformedness evaluation. Given that RM and phonological faithfulness constraints are not always antagonistic to each other (see section 2.4), markedness constraints have room to play a role in the computation in RMT. This interaction gives rise to effects of nonconcatenative allomorphy.

\subsubsection{Urbanczyk (1998)}

Urbanczyk (1998) gives an OT analysis of the continuative formation at issue. I critically review her analysis and clarify my idea along the line of the argument above. I focus attention on the distribution of the four nonconcatenative allomorphs. Urbanczyk (1998) makes several crucial assumptions in her analysis: (i) the continuative morpheme is essentially reduplicative $\mathrm{RED}_{\text {Cont. }}$ in nature, (ii) $\mathrm{RED}_{\text {Cont. }}$ is imperative in the sense that the morpheme itself demands reduplication, (iii) RM is violated whenever the continuative morpheme is not realized through reduplication, and (iv) there is a constraint called "DistinctStem" which mandates different output forms between noncontinuative and continuative forms. This constraint directly demands two corresponding forms to be phonologically distinct. The role of this 
constraint becomes clear from the tableau in (15), which is constructed according to her argument. *Struc-Syll militates against any single syllable, restricting the shape of the reduplicant to a monosyllable. The crucial comparison is between (15a) and (15c). According to Urbanczyk, the reason why (15c) is ruled out in favor of (15a) is that DistinctStem is infringed upon by (15c) by virtue of the fact that the continuative form is precisely the same as the noncontinuative base. In the same vein, the perfectly faithful form (16b) is also eliminated.

\begin{tabular}{|c|c|c|c|c|c|}
\hline & & /wi.qəs, RED Cont. $^{\text {/ }}$ & DistinctStem & *Struc-Syll & Max-BR \\
\hline & 畹 & wi-wə.qəs & & $* * *$ & $* * *$ \\
\hline b. & & wi.qa-wa.qas & & $* * * * !$ & $*$ \\
\hline c. & & wi.qəs & $* !$ & $* *$ & $* * * * *$ \\
\hline
\end{tabular}

\begin{tabular}{|c|c|c|c|c|}
\hline \multicolumn{3}{|c|}{ /mə.qət, $\mathrm{RED}_{\text {Cont. }} /$} & DistinctStem & Dep \\
\hline a. & $\log$ & ham.q'at & & $* *$ \\
\hline b. & & ma.qat & $* !$ & \\
\hline
\end{tabular}

Related to the assumption that the continuative morpheme is essentially reduplicative, the reason why DistinctStem is crucial becomes clear. Since RM is violated under Urbanczyk's analysis whenever reduplication fails, both (16a) and (16b) violate RM, as demonstrated in (17). This indicates that no stem modification is expected to be the optimal when reduplication is blocked, contrary to fact. The central claim of Urbanczyk (1998) is that reduplication is the norm to express the continuative morpheme, but when it creates a structure more marked than permissible, some other base modification enters the picture under the pressure of DistinctStem. 


\begin{tabular}{|c|c|c|c|c|}
\hline & & /mə.qət, RED ${ }_{\text {Cont. }}$ I & $\mathrm{RM}$ & Dep \\
\hline a. & : & ham.qat & $*$ & $* ! *$ \\
\hline b. & 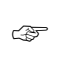 & mə.qat & $*$ & \\
\hline
\end{tabular}

However, this analysis has some serious drawbacks. First, DistinctStem misses the fundamental observation that noncontinuative and continuative forms are phonologically different because they have different morphemes. In other words, DistinctStem is insensitive to the fact that distinct forms are required for noncontinuatives and continuatives for a morphological reason. Although the surface effect of DistinctStem is roughly identical to that of RM, they are sharply distinct at the conceptual level. Thus, DistinctStem is unsatisfactory in the same way as antifaithfulness theory. By contrast, RM directly captures the idea that distinct forms are required by the presence of a new morpheme. This means that RM is satisfied by (17a) since $h$-prefixation can be taken to be the specific exponent of the continuative morpheme.

A more serious problem of Urbanczyk's (1998) analysis resides in her assumption that the continuative morpheme is essentially reduplicative. Urbanczyk (1998:656 fn.3) states that "It is hoped that the analysis developed here proves that a reduplicative analysis is possible." This statement is misleading. Even if her reduplicative analysis works, there is no language-internal evidence to support it. One might be led to her assumption by the nature of the morphosyntactic category under consideration. It is true that morphosyntactic functions such as continuatives, progressives, and repetitives are often denoted by reduplication in many languages, but this is not a universal principle. English progressives, which roughly correspond to the continuative morpheme in Upriver Halkomelem, do not take advantage of 
reduplication. Moreover, reduplication is quite productively and regularly used to express a variety of range of morphosyntactic categories other than those enumerated above. Thus, even this conjecture does not constitute a positive argument for Urbanczyk's assumption. That the continuative morpheme is essentially reduplicative is unmotivated, and thus, the reduplication-oriented analysis is built on stipulative assumptions.

I proposed that the continuative morpheme contains no phonological content, but a specific kind of exponent employed for the satisfaction of RM entirely depends on a constraint ranking. RM is operative as the driving force of the realization of the

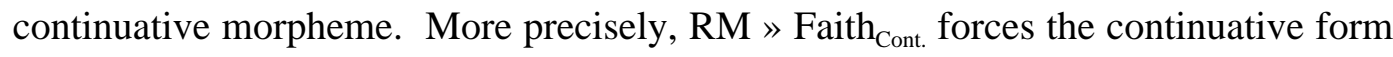
to undergo some phonological modification. RM simply demands the output representation to express the existence of all morphemes in the underlying representation. Importantly, this suggests that RM is sensitive to the input representation, desirably reflecting the intuition that differences between outputs of the continuative and the noncontinuative come from a difference of inputs. Second, the problem arising from Urbanczyk's assumption that the continuative is reduplicative disappears since the morpheme does not require any specific type of phonological exponence.

Again, my proposal has two significant consequences differentiating it from Urbanczyk's. First, RM is satisfied as long as the continuative morpheme receives some phonological exponence, as discussed in section 2.3. This means that (17a) satisfies RM in my system. As a corollary, reduplication is merely a potential but not the essential strategy to satisfy RM in the continuative formation. Urbanczyk's (1998) and my analyses are encapsulated in (18). 


\begin{tabular}{|c|c||c|}
\cline { 2 - 3 } \multicolumn{1}{c|}{} & Urbanczyk (1998) & Proposal here \\
\hline $\begin{array}{c}\text { What is the underlying morphemic } \\
\text { representation of continuative? }\end{array}$ & \{Continuative $\}$ & $\{$ Continuative $\}$ \\
\hline \hline Are morphemes process-specific? & YES & NO \\
\hline \hline $\begin{array}{c}\text { What requires different forms in two } \\
\text { distinct morphosyntactic categories? }\end{array}$ & DistinctStem & RM Faith \\
\hline \hline
\end{tabular}

\subsection{The Actual Aspect in Saanich}

As another case study, I examine the actual aspect formation in Saanich in this section. The data and descriptive generalization are based on Montler $(1986,1989)$. The actual aspect is opposed to the nonactual aspect, and signals that the action, state, or other reference of the predicate is actually occurring at an indicated time, roughly corresponding to the English progressive (Montler 1986:111). Nonactual aspect forms serve as the bases of the corresponding actual aspect forms. In this context, three nonconcatenative allomorphs are relevant: metathesis, reduplication, and :infixation, as schematically shown in (19).

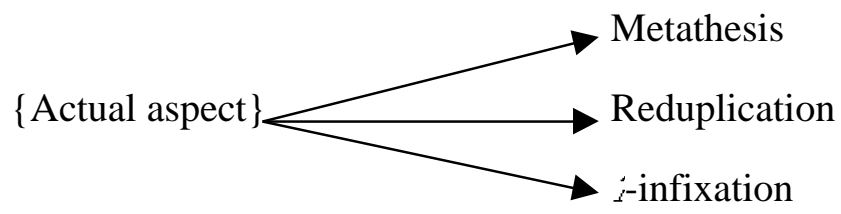

The situation looks quite similar to the nonconcatenative allomorphy involved in the continuative formation in Upriver Halkomelem. However, there is a significant difference between the two cases. Unlike $h$-prefixation in Upriver Halkomelem, the glottal stop is not epenthetic: it is phonological substance of the actual aspect morpheme. This suggests that the actual aspect morpheme is not contentless. In spite of this difference, I argue that the selection of the optimal allomorph in the actual 
aspect formation in Saanich is captured in exactly the same way as the continuative formation in Upriver Halkomelem. The example studied in this section is subsumed under the rubric of the general schema discussed in section 4.2.

In the rest of this section, I present the data of interest in section 4.4.1, and I present my analysis and discuss some important theoretical consequences in section 4.4.2. Finally, I critically review two earlier studies concerning the present issue (Montler 1989 and Stonham 1994) in section 4.4.3.

\subsubsection{Facts and Descriptive Generalization}

Concrete examples of metathesis, reduplication, and infixation found in the actual aspect formation are given in (20). In the data transcription, an apostrophe denotes that the preceding consonant is glottalized, and $\left[\mathrm{t}^{\mathrm{\theta}}\right]$ represents a voiceless interdental stop. Furthermore, primarily stressed vowels are underscored.

\begin{tabular}{|c|c|c|c|}
\hline Root & Nonactual & Actual & Gloss \\
\hline$q^{\prime} p^{\prime}$ & $q^{\prime} p^{\prime} \underline{\underline{\partial}} t$ & $q^{\prime} \underline{\partial p} p^{\prime t}$ & patch \\
\hline $\mathrm{sq}^{\prime}$ & sq'ât & sogq't & tear \\
\hline SX & SXât & sōxt & push \\
\hline $\int \mathrm{t} \int^{5}$ & $\int \mathrm{t} \int \underline{\partial t}$ & 〔ät]t & whip \\
\hline t's & t'sạt & t'âst & break \\
\hline $\mathrm{tq}^{\mathrm{w}}$ & $\mathrm{tq}^{\mathrm{w}} \underline{\underline{\partial}} \mathrm{t}$ & $\underline{t a} q^{w} t$ & tighten \\
\hline$\theta \mathrm{k}^{\prime \mathrm{w}}$ & $\theta \mathrm{k}^{\prime \mathrm{w}} \underline{\mathrm{a}} \mathrm{t}$ & $\theta \underline{\underline{a}} \mathrm{k}^{\prime} \mathrm{w}_{\mathrm{t}}$ & straighten \\
\hline$\theta x$ & $\theta x \underline{\underline{a}} \mathrm{t}$ & $\theta \underline{a} x t$ & shove \\
\hline$\chi^{\prime} \mathrm{k}^{\prime w}$ & $x^{\prime} \mathrm{k}^{\prime \prime \mathrm{w}} \underline{\underline{\partial}} \mathrm{t}$ & $\chi^{\prime} \underline{\partial}^{\prime \prime} \mathrm{t}$ & put out \\
\hline $\mathrm{t}^{\prime \prime} 4 ə \mathrm{k}$ & $t^{\prime \prime} 4 \underline{a} k$ & $\mathrm{t}^{\prime \theta} \underline{\underline{\partial}} \mathrm{Ak}$ & pinch \\
\hline$\tilde{X}^{\prime}$ рәх & $\tilde{\lambda}^{\prime} \mathrm{p} \underline{\mathrm{x}}$ & $\chi^{\prime} \underline{\text { opx }}$ & scatter \\
\hline
\end{tabular}

b. Reduplication

\begin{tabular}{|c|c|}
\hline qen' & qen' $+(C)$ \\
\hline$t^{1 \theta} e ?$ & $\mathrm{t}^{\prime \theta} \underline{\mathrm{e}} ?+(\mathrm{C})$ \\
\hline$q^{\mathrm{w}} \mathrm{al}$ & $\mathrm{q}^{\mathrm{w}} \mathrm{a} \mathrm{l}+(\mathrm{C})$ \\
\hline $\mathrm{k}^{\mathrm{w}} \mathrm{ul}$ & $\mathrm{k}^{\mathrm{w}} \underline{\mathrm{u}} \mathrm{l}+(\mathrm{C})$ \\
\hline $4 i k^{\prime w}$ & $4 \mathrm{ik}^{\prime \mathrm{w}}+(\mathrm{C})$ \\
\hline qew & $\mathrm{qe} w+(\mathrm{C})$ \\
\hline tap' & tapp'+(C) \\
\hline yq & $\mathrm{t}\lceil\underline{a k}+(\mathrm{C})$ \\
\hline
\end{tabular}

qe-qan' steal

$\mathrm{t}^{\mathrm{t}} \mathrm{e}-\mathrm{t}^{\mathrm{t} \theta} \mathrm{\partial}$ ride

$\mathrm{q}^{\mathrm{w}} \underline{\mathrm{o}}-\mathrm{q}^{\mathrm{w}} \mathrm{al}$ say

$\mathrm{k}^{\mathrm{w}} \underline{\mathrm{u}}-\mathrm{k}^{\mathrm{w}} \mathrm{el} \quad$ school

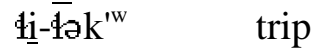

qe-qow rest

ła--\$op' eat

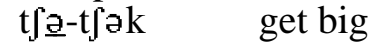




\begin{tabular}{|c|c|c|c|c|}
\hline \multirow{6}{*}{ c. 2-infixation } & $2 \mathrm{it}^{1 \mathrm{\theta}}$ & $2 \mathrm{it}^{\prime \theta}+\mathrm{V}$ & $2 i t^{\prime \theta}$ & get dressed \\
\hline & ?ets & ?et $\int+V$ & ?e?t & wipe \\
\hline & t jaq'w & $\mathrm{t} \int \underline{a}^{\prime \prime w}+\mathrm{V}$ & $\mathrm{t} \int \underline{a} 2 q^{\prime w}$ & sweat \\
\hline & Piten & ii.ton+V & ịi.ton & eat \\
\hline & weqas & we.qas & we?.qəs & yawn \\
\hline & Zamət & 2a.mot & 2a?.mat & sleep \\
\hline
\end{tabular}

First, I briefly sketch phonological aspects of relevance. In metathesis, a vowel and the immediately preceding consonant undergo reversal. Second, in reduplication, the initial $\mathrm{CV}$ of the nonactual aspect base is uniformly copied, creating a CV reduplicant. The base-initial vowel is concomitantly reduced to a schwa, which is similar to the vowel reduction process observed in reduplication in the continuative formation in Upriver Halkomelem. The reason behind vowel reduction is clear in Saanich. Montler (1986:28, 1989:100) documents that unstressed vowels are all reduced to a schwa. This correlates with the strong pattern in Saanich that all vowels in a word are schwas except the vowel bearing stress. Finally, -infixation appears right after stressed vowels. I do not discuss these phonological aspects, assuming that independent interactions of constraints not discussed here yield them.

Second, consider the distribution of the three allomorphs, which is the central concern here. The examples in (20) exhibit a seemingly complicated distributional pattern of metathesis, reduplication, and infixation, but they indeed receive straightforward descriptive generalization. First, as in (20a), metathesis is taken when a base form is CCVC, where the final VC may be a suffix or part of the root. Second, as in (20b), reduplication is employed when (i) a root is CVC followed by nothing or a C-initial suffix, and (ii) a root is $\mathrm{CC}$, where a schwa is epenthesized between the two root consonants to create the nonactual aspect form. Finally, as in (20c), glottal stop infixation appears elsewhere. The three kinds of phonological exponence are therefore in complementary distribution, supporting the idea that they are allomorphs. 


\subsubsection{Analysis}

The analysis starts by considering the morpheme-internal representation of the actual aspect. Given the discussion in chapter 2, metathesis and reduplication are obtained when RM outranks Linearity and Integrity respectively. Thus, the morpheme needs no phonological material to trigger metathesis and reduplication. The question is whether the glottal stop needs to be explicitly encoded as the phonological substance of the actual aspect morpheme. The answer to this question is positive. Montler (1986:28) reports $h$-epenthesis to break up a cluster of a schwa and a full vowel, but no -epenthesis is documented, justifying that the glottal stop is not the default epenthetic consonant in Saanich. This suggests that the existence of the -allomorphy cannot be derived without an underlying specification under the normal assumption that only the default segment serves as the epenthetic element. The morphemic structure of the actual aspect thus looks as in (21). This constitutes a stark contrast with the continuative morpheme in Upriver Halkomelem which is entirely contentless.

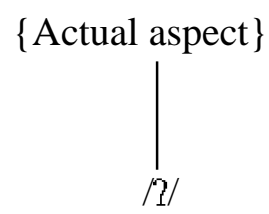

Given that metathesis, reduplication, and infixation are relevant, the following three faithfulness constraints come into play: Linearity Actual, Integrity $_{\text {Actual, }}$, and Contiguity $_{\text {Actual. }}$. These constraints bear the actual aspect morphemic marking. This morphosyntactic specification is important because promiscuous metathesis, reduplication, and i-infixation would take place otherwise regardless of morphosyntactic categories. In the analysis below, I implicitly assume that all other 
faithfulness constraints are undominated. Crucial markedness constraints are *Complex(onset) and *Complex(coda) (Prince and Smolensky 1993). Besides these constraints, RM is also indispensable.

(22) is the analysis of metathesis cases in (20a) (e.g., /q'p'at/ $\rightarrow$ [q'əp't]). The crucial observation is that glottal stop infixation does not resolve the offending consonantal sequence violating undominated *Complex(onset). The only way to satisfy the constraint is to employ metathesis. One candidate not included in (22) is [q'əq'.p'at], where the initial [q'ə] is the reduplicant. The fault of this form is that the reduplicated segments are not contiguous, ruled out by Contiguity-BR. Under the present analysis where the glottal stop is part of the underlying phonological material, Max is clearly violated by the optimal candidate. The question is why [q'at], for instance, does not surface. From the facts illustrated above, it should be evident that a root segment never undergoes deletion. This observation is captured by Faith-Root» Faith-Affix (or more specifically, Max-Root » Max-Affix), granting faithfulness priority to roots over affixes (McCarthy and Prince 1995; Urbanczyk 1996; Alderete 1999).

\begin{tabular}{|c|c|c|c|c|c|c|c|}
\hline & /q'p'at, ?/ ${ }_{\text {Act. }}$ & $\mathrm{RM}$ & $\begin{array}{c}* \text { Compl } \\
\text { (ons) }\end{array}$ & $\begin{array}{c}* \text { Compl } \\
\text { (coda) }\end{array}$ & Lin & Integ & Contig \\
\hline $\mathrm{a}$ & q'p'at & $* !$ & $*$ & & & & \\
\hline $\mathrm{b}$ & q'p'ə?t & & $* !$ & $*$ & & & $*$ \\
\hline & q'op't & & & $*$ & $*$ & & \\
\hline d & q'a-q'əp't & & & * & $*$ & $* ! *$ & \\
\hline
\end{tabular}

Second, (23) is a case of reduplication. Candidate (23a) is ruled out by RM. The optimal form (23e) violates only Integrity ${ }_{\text {Actual }}$, but candidates (23b-d) fail to 
satisfy at least one higher ranked constraint. Candidates (23b) and (24c) violate *Complex(coda), and (23d) violates *Complex(onset). The crucial difference between (20a) and (20b) is what constitutes the initial two segments. In (20a), the initial two segments are CC, but in (20b), they are CV. Thus, base forms in (20a) contain offending consonantal concatenation against *Complex(onset), but those in (20b) do not. This is the reason why metathesis is not chosen in (20b) because it creates a violation of a syllable markedness constraint, either $*$ Complex(onset) or *Complex(coda), as seen from $(23 \mathrm{c}, \mathrm{d})$. Rather, reduplication is the best because it does not produce any violation of the markedness constraints. The same analysis holds of cases where a CVC root is followed by a consonant-initial suffix. Again, Max is violated by the optimal candidate due to its underparsing of the glottal stop affiliated with the continuative morpheme. Max-Root » Max-Affix is essential here too.

\begin{tabular}{|ll||c:c|c|c|c|c|}
\hline & /qen', Act. & RM & $\begin{array}{c}* \text { Compl } \\
\text { (ons) }\end{array}$ & $\begin{array}{c}\text { *Compl } \\
\text { (coda) }\end{array}$ & Lin & Integ & Contig \\
\hline \hline a. & qen' & $* !$ & & & & & \\
\hline b. & qe?n' & & & $* !$ & & & $*$ \\
\hline c. & eqn' & & & $* !$ & $*$ & & \\
\hline d. & qn'e & & $* !$ & & $*$ & & \\
\hline e. & & & & & $* *$ & \\
\hline
\end{tabular}

Finally, let us consider cases of glottal stop infixation in (20c). As mentioned above, a glottal stop is infixed when initial CVC is followed by a vowel, whether it is part of a root or the first segment of a suffix. A representative tableau is provided in (24). (24a) is immediately ruled out because of its fatal violation with respect to 


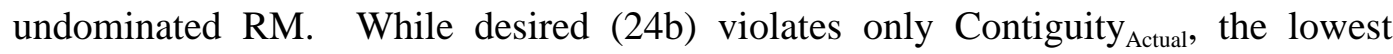
ranked constraint, $(24 \mathrm{c}-\mathrm{e})$ violate at least one higher constraint. Thus, it is clear that $(24 b)$ is the winner. Of particular interest here is the comparison between $(24 b)$ and (24e). Comparing (20b) and (20c), the crucial difference is the segment that follows the CVC: a consonant in (20b) but a vowel in (20c). Infixation creates a consonant cluster in (20b), but it does not in (20c). Contiguity $_{\text {Actual }}$ is crucially dominated by Integrity $_{\text {Actual }}$. This means that, everything being equal, infixation costs less than reduplication. This is why glottal stop infixation is the best way of realizing the actual aspect morpheme.

\begin{tabular}{|c|c|c|c|c|c|c|c|c|}
\hline & & /weqas, ?/ & RM & $\begin{array}{c}* \text { Compl } \\
\text { (ons) }\end{array}$ & $\begin{array}{c}* \text { Compl } \\
\text { (coda) }\end{array}$ & Lin & Integ & Contig \\
\hline a. & & weqas & $* !$ & & & & & \\
\hline & 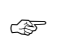 & we?.qas & & & & & & * \\
\hline c. & & wqe.əs & & $* !$ & & * & & \\
\hline d. & & ew.qas & & & & $* !$ & & \\
\hline e. & & we-wa.qas & & & & & $* ! *$ & \\
\hline
\end{tabular}

Summarizing the analysis above, the relevant constraint ranking is given in (25). This constraint hierarchy shares the essential characteristics of the general proposal in section 4.2 with the continuative formation in Upriver Halkomelem: operations involving a faithfulness constraint that is not ranked the lowest are possible only when some markedness constraint blocks the operation which violates the lowest ranked faithfulness constraint. In other words, various interactions between markedness and faithfulness constraints make the multiple range of nonconcatenative allomorphs possible, supporting the argument in section 4.2. What 
is remarkable in this constraint ranking is the fact that Contiguity Actual $_{1}$ is accorded the lowest status because it nicely reflects the observation that -infixation is the elsewhere case, and therefore, is the default.

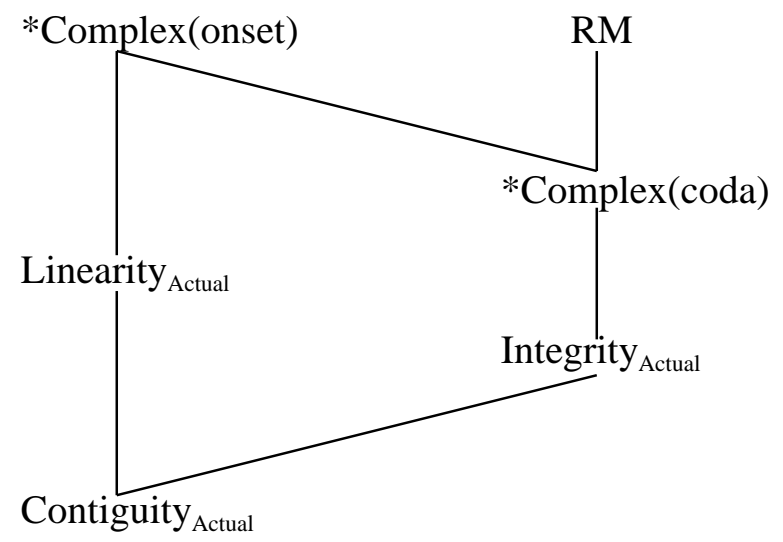

The analysis here has two important theoretical consequences. The most striking one concerns the morphemic representation of the actual aspect. As in (21), this morpheme inherently contains the glottal stop segment, but it fails to surface when metathesis or reduplication is selected. The failure of the surface realization of the underlying phonological substance is nothing surprising given that metathesis, reduplication, and i-infixation are in complementary distribution. The success of the proposed OT analysis is that this is explained naturally. -infixation is prevented when it creates a highly marked structure. The surface non-realization is a strategy to eschew markedness. Markedness-based systems like OT thus provide a principled reason for this. By contrast, the peculiarity remains a mystery in approaches with no or little markedness consideration. i-infixation should suffice to satisfy RM, so why does Saanich decide to bother to give up using the underlying material and to modify the phonological shape of the base form? To answer this question, syllable structure constraints imposed on phonological representations or some other equivalent would 
be needed additionally, but this is what the proposed OT analysis implements directly.

A second point has to do with a broader theoretical perspective. I discussed in chapter 2 that there should not be any formal distinction between concatenative and nonconcatenative morphology. The actual aspect formation in Saanich represents a good empirical case for this claim because the three allomorphs are a mixture of concatenative and nonconcatenative morphology. Were the two terminologically distinguished types of morphology independent of each other and governed by completely different linguistic principles, this kind of mixture is completely incomprehensible. Under the view that there exists no formal factor which differentiates them (except the presence or absence of some phonological content associated with the given morpheme), the coexistence of concatenative and nonconcatenative allomorphs is unsurprising.

\subsubsection{Montler (1989) and Stonham (1994)}

Montler (1989) and Stonham (1994) provide an overall analysis of the actual aspect formation in Saanich. Their analyses are both couched in a derivational framework, but their concrete proposals are radically different from each other. In this section, I critically review both of them, and argue that nether of them is satisfactory.

The central claim of Montler (1989) is that the actual aspect formation is templatic, the template being CVCC. This template is operative only word-initially such that the initial four segments of all actual aspect forms must conform to the templatic restriction. This templatic approach directly accounts for the metathesis and -infixation examples in $(20 \mathrm{a}, \mathrm{c})$. But this analysis encounters a number of 
problems, both empirical and conceptual. Empirically, the reduplication examples in (20b) cannot be explained. Recall that the reduplicative allomorph surfaces when the nonactual aspect base is CVC optionally followed by a C-initial suffix. Suppose that a CVC base is followed by such a suffix. The base form is already CVCC in this case and accords with the template. The question is why reduplication takes place. Note that it prevents template satisfaction. By contrast, when no such suffix exists, the base form is CVC, but again, reduplication does not make any contribution to the accomplishment of the CVCC template. In effect, reduplication simply does not make sense in terms of the CVCC templatic approach. Montler (1989:101) states "...if all that is there is CVC, reduplicate the first C...", but this statement is wrong because the reduplicant is constantly $\mathrm{CV}$. The vowel quality of the reduplicant is the same as that of the copied segment, so this point is not refutable.

This analysis has a conceptual difficulty as well. Since McCarthy and Prince (1986), a growing body of arguments has been developed arguing that templates are prosodically defined. In the infixation cases, it is obligatory that the base-initial CVC is followed by a vowel, either the continuation of the base or a suffix. This suggests that the second consonant in a nonactual aspect form serves as the onset of a syllable whose nucleus position is occupied by the base-second or suffix-initial vowel, indicating that the CVCC template is separated into two syllables as in [CVC.C]. This is against arguments in favor of the prosody-based view of templates.

These empirical and conceptual problems would not be surmountable. Furthermore, Montler assumes a floating [+constricted glottis] feature as the source of -infixation, but it remains a mystery why it does not appear as a secondary articulation, glottalizing a base consonant when there is such room (e.g., *[q'əp't'] 
from [q'op't]) because Saanich allows for numerous glottalized phonemes. In other words, the floating feature analysis fails to account for the reason why it is not realized in the surface representation when reduplication or metathesis is employed. This is a serious pitfall since this means that the analysis cannot capture the fundamental observation that metathesis, reduplication, and $;$-infixation are in complementary distribution (i.e., allomorphemic). This problem, together with the aforementioned empirical and conceptual difficulties, further weakens the plausibility of the analysis proposed by Montler (1989). I conclude that the templatic analysis must be rejected.

Stonham (1994) develops a prosodic analysis. The essential claim is that the actual aspect morpheme carries a mora, a prosodic unit whose phonological content is not specified. This mora has the effect of augmenting the nonactual aspect form by one mora in the actual aspect formation (see Davis and Ueda 2001 for an OT analysis along this line). Starting with metathesis cases in (21a), he argues that it is in some sense the simplest and most economical from a language-based view since it does not add any new phonemic material (Stonham 1994:175). However, it is not clear why segmental reversal is less costly than epenthesis, for instance. Schwa epenthesis is regularly and productively used in Saanich to break up a consonant cluster in certain environments (Montler 1986:30), so an obvious possibility is to augment the nonactual forms in (21a) by schwa epenthesis between the two offending consonants. In the OT conception, metathesis and epenthesis incur different faithfulness constraint violations, so it is not valid to evaluate the economy of the two distinct processes in terms of the presence or absence of a new segment. Stonham (1994:175) discusses the possibility of vowel lengthening as a way of augmenting nonactual aspect bases. 
He denies it because vowel length is not contrastive in Saanich, but phonological contrasts are not a necessary factor in the evaluation of morpheme realization.

Stonham's (1994) analysis crucially departs from Montler's (1989) in that he assumes no concrete phonological substance as the driving force of $i$-infixation. This gives rise to a problem. Given the absence of phonological substance, it is expected that the epenthesized segment is the default segment in the language. As stated in the previous section, Montler (1986:28) reports $h$-epenthesis to break up a cluster of a schwa and a full vowel, but no i-epenthesis is documented. This indicates that the default epenthetic consonant is [h].

The various problems discussed above show that Stonham (1994) presupposes the possible range of phonological changes available for the actual aspect formation, putting the cart before the horse. Furthermore, his analysis crucially relies on CVsegregation (McCarthy 1979, 1981), but this theoretical device is not independently motivated language-internally, so this is a stipulation not necessary anywhere else in Saanich phonology and morphology.

The review here suggests that neither Montler (1989) nor Stonham (1994) is satisfactory. Despite the fact that they attempt to reduce the three nonconcatenative allomorphs to a single source, their analyses are undesirable.

\subsection{The Incomplete Phase in Rotuman}

Following the discussion of the two Salishan languages, I examine Rotuman, a central Oceanic language, which has no genetic relationship with Salishan languages. Of interest here is the incomplete phase formation. Complete phase forms are phonologically the same as stem forms, but there are indications that the complete 
phase serves as the base of the incomplete phase since some prosodic faithfulness constraints are active in the latter formation. As discussed in section 2.3, bare stems are basically taken to be the bases of word formations, but it does not preclude the possibility that some morphosyntactic category is derived from another (cf. deverbal nouns in Icelandic). I take the incomplete phase as the base of the incomplete phase.

The selection of the appropriate phase in a given context is governed by various principles outside phonology (Churchward 1940:88-89). The phonology involved in this word formation is similar to the two cases studied in the previous sections in that various nonconcatenative base modificational strategies are attested depending on the base form. This phase alternation is comprehensively described by Churchward (1940) and theoretically studied quite extensively in the earlier literature (Haudricourt 1958ab; Biggs 1959, 1965; Milner 1971; Cairns 1976; Saito 1981; van der Hulst 1983; Janda 1984; McCarthy 1986b, 1989, 2000c; Mester 1986; Besnier 1987; Hoeksema and Janda 1988, Odden 1988; Anttila 1989; Weeda 1992; Blevins 1994). All content words exhibit the phase alternation. Numerous nonconcatenative base modifications are observed depending on the base shape, as in (26). An obvious difference from the two previous cases is that the incomplete phase sometimes fails to receive phonological exponence. I argue that no surface realization appears when any effort to satisfy RM results in a violation of some undominated constraint.

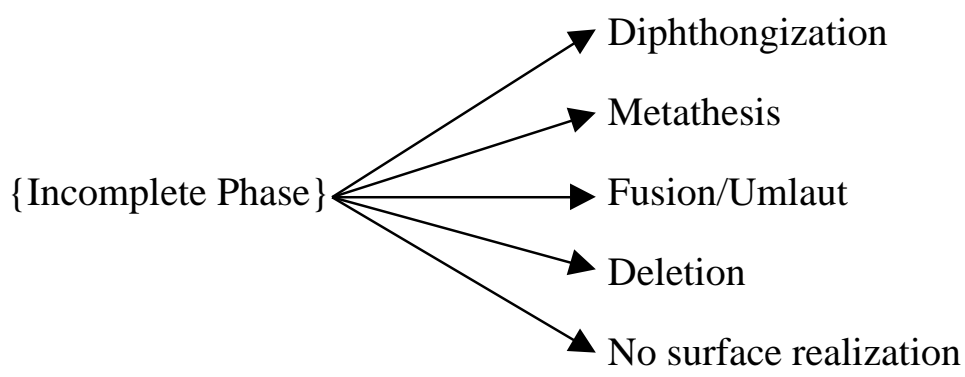


Another significant characteristic which differentiates the incomplete phase formation from the Salishan examples is that it is truly templatic in the sense that various base deformative strategies yield a certain prosodic shape word-finally. This point is especially important for the following discussion since the templatic effect is strictly emergent in my analysis, a by-product of interactions of constraints rather than a requirement forced by a templatic constraint per se. But the incomplete phase formation receives fundamentally the same analysis as the Salishan cases.

This section is mapped out as follows. I present the crucial set of data in section 4.5.1 and provide descriptive generalization. Section 4.5.2 is devoted to presenting my analysis. Finally, in section 4.5.3, I provide a critical review of McCarthy (2000c) among many other earlier works cited above. It attempts to offer a comprehensive OT analysis of the morphology and phonology involved in the incomplete phase formation process. His analysis is quite similar to mine, but the key constraint operative as the driving force of various base modifications is fundamentally different.

\subsubsection{Facts and Descriptive Generalization}

In this section, I present the basic data to be accounted for. As in (27), five varieties of base modification are relevant, including lack of overt surface realization. They are exemplified in (27). Although these examples seem to be fairly complex in terms of their distribution, they receive reasonably straightforward generalization. First, diphthongization occurs as in (27a) when the complete phase ends in a vowel cluster (heavy or bimoraic diphthongs are denoted by the ligature). This point is discussed in the next section. Unless a vowel sequence is found word-finally, diphthongization is 
never employed. Metathesis of the final CV takes place when the result is a vowel sequence with rising sonority, where low and high vowels have the highest and the lowest sonority value respectively, mid vowels in-between. This is exemplified in (27b). Third, as in (27c), vowel fusion/umlaut occurs when metathesis is not available because of the sonority requirement mentioned above. When the relevant two vowels are identical, they are simply fused into a single segment, but when their qualities are not the same, the first vowel is umlauted. Since the source of umlaut comes from the front property of a word-final vowel, it is a prerequisite that the final vowel of a complete phase form is front. The simple fusion cases as in [hanuj] have been analyzed as final vowel deletion by many earlier authors (Besnier 1987; Blevins 1994; McCarthy 2000c), but I argue that fusion is correct. Word-final vowel deletion is independently required as in (27d). In (27d), metathesis is not an eligible option because the first vowels are more sonorous than the word-final ones. Furthermore, umlaut is not an option either since the first vowels are already front. Finally, no surface realization appears as in (27e) if the base-final vowel is long.

$\begin{array}{lll}\text { a. } & \text { Diphthongization } & \\ \text { Complete Phase } & \text { Incomplete Phase } & \text { Gloss } \\ \text { ?ea } & \text { ?ea } & \text { to say } \\ \text { foa } & \text { foa } & \text { coconut scraper } \\ \text { ?io } & \text { Tio } & \text { to see } \\ \text { kelea } & \text { kelea } & \text { to look } \\ \text { pupui } & \text { pupui } & \text { floor } \\ \text { lelei } & \text { lelei } & \text { good }\end{array}$

b. Metathesis

$\begin{array}{lll}\text { Complete Phase } & \text { Incomplete Phase } & \text { Gloss } \\ \text { pure } & \text { puer } & \text { to rule } \\ \text { rito } & \text { riot } & \text { to glitter } \\ \text { loja } & \text { loan } & \text { toward the interior of island } \\ \text { ulo } & \text { uol } & \text { seabird sp. } \\ \text { piko } & \text { piok } & \text { lazy }\end{array}$


c. Fusion/Umlaut

$\begin{array}{lll}\begin{array}{l}\text { Complete Phase } \\ \text { hanuju }\end{array} & \begin{array}{l}\text { Incomplete Phase } \\ \text { hanuj }\end{array} & \begin{array}{l}\text { Gloss } \\ \text { tale }\end{array} \\ \text { toto } & \text { tot } & \text { blood } \\ \text { nono } & \text { non } & \text { grip } \\ \text { kamata } & \text { kamat } & \text { to begin } \\ \text { hose } & \text { hös } & \text { oar } \\ \text { fuli } & \text { fül } & \text { deaf } \\ \text { futi } & \text { füt } & \text { to pull } \\ \text { mose } & \text { mös } & \text { to sleep }\end{array}$

d. Deletion

$\begin{array}{lll}\text { Complete Phase } & \text { Incomplete Phase } & \text { Gloss } \\ \text { la?o } & \text { la? } & \text { to go } \\ \text { asu } & \text { as } & \text { smoke } \\ \text { haju } & \text { hay } & \text { to awake } \\ \text { hasu } & \text { has } & \text { gall bladder } \\ \text { rako } & \text { rak } & \text { to imitate }\end{array}$

e. No surface realization

$\begin{array}{lll}\text { Complete Phase } & \text { Incomplete Phase } & \text { Gloss } \\ \text { rii } & \text { rii } & \text { house } \\ \text { ree } & \text { ree } & \text { to do } \\ \text { sikad } & \text { sikaa } & \text { cigar }\end{array}$

The descriptive generalization is significant in terms of the harmonic scale of the nonconcatenative allomorphs, as discussed by McCarthy (2000c). Setting aside (27e) where no exponent realizes the incomplete phase, the four processes are hierarchically ranked in the order of preference. The context where diphthongization is a valid process is strictly limited to cases where the word-final portion contains a vowel sequence. A segmentally affected process is demanded in all other environments. The environment of metathesis is next stringently restricted. Fusion/Umlaut is employed when metathesis is blocked by the sonority condition. In the same vein, fusion/umlaut is more preferred to deletion. In effect, deletion is the last resort strategy to achieve an explicit phonological expression of the incomplete phase morpheme. Taking (27e) into account, the five allomorphs receive the 
harmonic scale in (28) where the order is given in decreasing harmony from left to right. This scale is based on faithfulness considerations. Note that simple fusion is more harmonic than umlaut since the latter incurs additional faithfulness violations. This harmonic scale is a direct and important input to the analysis in the next section.

No realization $\rightarrow$ Diphthongization $\rightarrow$ Metathesis $\rightarrow$ Fusion $\rightarrow$ Umlaut $\rightarrow$ Deletion (most harmonic)

(least harmonic)

\subsubsection{Analysis}

In this section, I present an analysis along the lines discussed thus far. Since metathesis, fusion, umlaut, and deletion are relevant, Linearity, Uniformity, and Max are the pertinent constraints. Setting various prosodic faithfulness constraints aside, the constraint ranking in (29) is established based on the harmonic hierarchy in (28).

$$
\mathrm{RM} \gg \mathrm{Max}_{\text {Incomplete }} \text { " Linearity } \text { Incomplete }_{\text {, Uniformity }} \text { Incomplete } \text { * *Heavy-Diphthong }
$$

Since final vowel deletion is the least preferred, the faithfulness constraint prohibiting segmental deletion (i.e., Max) is ranked the highest among the relevant faithfulness constraints. Metathesis violates only Linearity while umlaut violates both Linearity and Uniformity because umlaut is a consequence of fusion of the last two vowels, involving segmental reversal of the word-final vowel and the preceding vowel. In other words, metathesis incurs only a subset of violations of Umlaut. Thus, the relative harmony between metathesis and umlaut can be accounted for without manipulating a crucial ranking between Linearity and Uniformity. RM is the driving force of nonconcatenative base changes, so it must be ranked over all the relevant faithfulness constraints. Considering diphthongization which involves no 
segmental changes, I assume *Heavy-Diphthong. It may be more plausibly substituted by a prosodic faithfulness constraint, but it suffices for our purposes. I omit the morphosyntactic marking on the faithfulness constraints subsequently.

Two more pieces of background information are necessary. A first one concerns the prosodic structure of the complete and the incomplete phases, in particular, in order to motivate diphthongization in (27a). The following discussion regarding this issue owes a debt to McCarthy (2000c). It is argued at length that the various nonconcatenative base modifications involve the prosodic changes in wordfinal position, as schematically shown in (30). He justifies the prosodic structures mainly based on stress assignment facts. The crucial distinction between these two phases is that the complete phase ends in a disyllabic foot whereas the word-final foot is constantly a bimoraic monosyllable in the incomplete phase.

Diphthongization

Metathesis/Fusion/Umlaut/Deletion

Complete

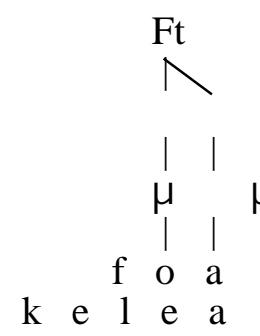

Incomplete

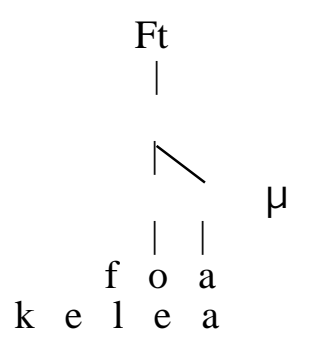

Incomplete

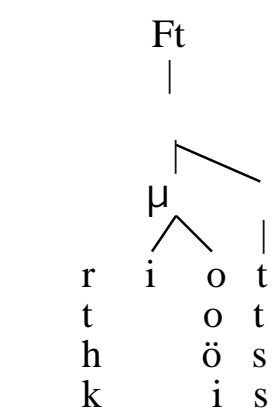

The only exception to the word-final disyllabic foot generalization is cases where the word-final vowel is long. The complete phase contains a heavy syllable word-finally in such cases, as represented in (31). This conforms to the moraic theory in which long vowels are invariably bimoraic (Hyman 1985; McCarthy and Prince 
1986; Hayes 1989). The important consequence is that no phonological distinction is made between the two phases in such cases. Otherwise, the complete phase always has a word-final foot consisting of two light syllables. This observation is significant because it motivates the diphthongization process in (27a).

(31) No surface realization

Complete $=$ Incomplete

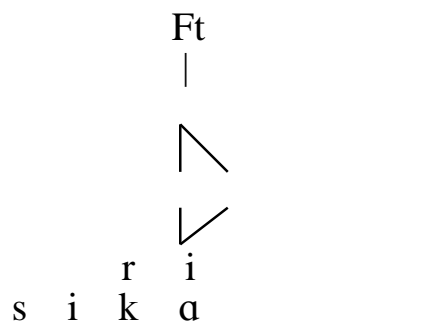

As a final background before presenting my analysis, I introduce several additional constraints to facilitate the subsequent argument. The relevant constraints are provided in (32).

(32) a. Light-Diphthong:

The sonority value must rise for a light (or monomoraic) diphthong.

b. $\quad * \sigma_{\mu \mu \mu}$ :

Trimoraic syllables are prohibited.

c. $\quad$ RHType $=$ Trochaic:

Feet are trochaic.

d. $\quad$ Align-R(Ft,PrWd):

Feet are aligned to the right edge of a prosodic word.

e. Head-Match:

If $\alpha$ is the prosodic head of the word and $\alpha \Re \beta$, then $\beta$ is the prosodic head of the word.

f. Weight-Ident:

If $\alpha \Re \beta$, and if $\alpha$ is monomoraic, then $\beta$ is monomoraic, and if $\alpha$ is bimoraic, then $\beta$ is bimoraic. 
A brief explanation of each of these constraints is in order. Light-Diphthong dictates that tautosyllabic light diphthongs must have a sequence of vowels such that the second one is more sonorous than the first one (Rosenthall 1994). This is never violated in Rotuman, so Light-Diphthong is undominated. Next, $* \sigma_{\mu \mu \mu}$ literally bans trimoraic syllables, which is also faithfully obeyed with no exception. Third, the rhythmic type constraint (Prince and Smolensky 1993) and the foot alignment constraint (McCarthy and Prince 1993a) together derive the effect of the moraic trochee in Rotuman. Permissible foot structures consist either of two light syllables (where the first syllable carries stress) or of a single heavy syllable. (32c) and (32d) together require that a moraic trochee is aligned at the right edge of a prosodic word. The property of a moraic trochee holds of both the complete and the incomplete phases. Head-Match requires head correspondence between the two phases. Thus, it is an output-output correspondence constraint comparing the two phases because prosodic information is reliably present only in output forms given the conception of richness of the base (Prince and Smolensky 1993; Smolensky 1996; Kurisu 2000c). Since the head or the stressed vowel in the complete phase is carried over to the incomplete phase, this constraint can be considered to be undominated for the present purposes. But it must be dominated by the rhythmic type and alignment constraints, as discussed by McCarthy, when we consider stress assignment in the context of the richness of the base hypothesis. Finally, Weight-Ident in (32f) is a faithfulness constraint militating against vowel lengthening and shortening. This constraint needs to be ranked fairly low to ensure the strict obedience to the bimoraic word minimality requirement in connection with the richness of the base hypothesis (see also Blevins 1994). We are dealing with the phase alternation involving only surface-to-surface 
correspondence relations, so we can safely assume that Weight-Ident is undominated too for our purposes. In effect, all the constraints in (32) can be assumed inviolable so that many potential candidates can be removed from the following analysis.

Given the background above, I consider the examples in (27) in the order presented there. Beginning with diphthongization, a tableau is given in (33). There is not much worth remarking here since *Heavy-Diphthong is ranked the lowest. Other conceivable processes such as metathesis, umlaut, and deletion are suboptimal because they incur one or more higher ranked violation. A potential candidate not included in (33) is [pu.pui], syllabifying the last three segments tautosyllabically as a light diphthong. But this candidate fatally violates Light-Diphthong. Since *HeavyDiphthong is ranked the lowest and therefore does not exhibit any interesting interactions with other constraints, I leave it out in the subsequent tableaux.

\begin{tabular}{|ll||l|l|l|c|c|}
\hline & /pu.pu.i/Incompl. & RM & Max & Lin & Unif & $\begin{array}{c}* \text { Heavy- } \\
\text { Diphthong }\end{array}$ \\
\hline a. & pu.pu.i & $* !$ & & & & \\
\hline b. & pu.pui & & & & & $*$ \\
\hline c. & pu.pi.u & & & $* !$ & & \\
\hline d. & pu.pü & & & $* !$ & $*$ & \\
\hline e. & pu.pu & & $* !$ & & & \\
\hline
\end{tabular}

Second, consider metathesis in (27b). It is strictly regulated by LightDiphthong, but the examples in (27b) satisfy the requirement. As demonstrated in (34), no morphemic realization (34a) and deletion (34d) violate a constraint ranked over Linearity violated by the optimal form, and umlaut (34c) incurs a gratuitous violation of Uniformity. Two more plausible candidates exist: [pu.er] and [puer]. In 
[pu.er], the stress should fall on the last vowel as in [pu.ér] under duress of the rhythmic type constraint in (32c) and the foot alignment constraint in (32d). However, it infringes on undominated Head-Match in (32e). By contrast, [puer] is ruled out by the constraint against trimoraic syllables in (32b). The constraints in (32) are all ranked over RM, so by transitivity, over Linearity. This shows that the two additional candidates are eliminated for independent reasons. The reason why diphthongizaion is eligible only when the complete phase form ends in a vowel cluster becomes clear now. Since coda consonants are consistently a weight bearing unit, diphthongization in other contexts necessarily results in a violation of $* \sigma_{\mu \mu \mu}$.

\begin{tabular}{|ll||l|l|l|l|}
\hline & /pu.re/ Incompl. & RM & Max & Lin & Unif \\
\hline \hline a. & pu.re & $* !$ & & & \\
\hline b. & puer & & & $*$ & \\
\hline c. & pür & & & $*$ & $* !$ \\
\hline d. & pur & & $* !$ & & \\
\hline
\end{tabular}

Third, let us turn to simple fusion cases, a subset of (27c). Simple fusion occurs when the last two vowels are exactly identical. Thus, potentially conceivable candidates can be quite narrowed down from the beginning. For instance, umlaut is not a plausible option, and diphthongization is untenable for the reason stated above. However, as illustrated in (35), simple fusion cases are interesting since final vowel deletion is another possible interpretation of those examples, as actually done by Besnier (1987), Blevins (1994), and McCarthy (2000c). Comparing (35b) and (35c), the fusion candidate turns out to be more harmonic than the deletion candidate. As discussed above and exemplified shortly below, Max » Linearity and Max » 
Uniformity are independently needed to explain the umlaut examples, the remaining subset of (27c). This justifies to group the cases I call simple fusion with umlaut rather than with deletion.

\begin{tabular}{|ll||c|c|c|c|}
\hline & /ha.nu $1 . \mathrm{ju}_{2} /$ Incompl. & $\mathrm{RM}$ & $\mathrm{Max}$ & Lin & Unif \\
\hline a. & ha.nu $1 . \mathrm{ju}_{2}$ & $* !$ & & & \\
\hline b. & ha.nu $_{1,2 \mathrm{j}}$ & & & $*$ & $*$ \\
\hline c. & ha.nu $\mathrm{j}$ & & $* !$ & & \\
\hline
\end{tabular}

In order to motivate Max » Linearity and Max »Uniformity, consider the umlaut examples. As shown in (36), Max outranks both Linearity and Uniformity to obtain umlaut because it is never expected to appear otherwise. Three candidates are omitted in (36): [moes], [moes], and [mo.es]. Light-Diphthong, ${ }^{*} \sigma_{\mu \mu \mu}$, and HeadMatch rule them out respectively.

\begin{tabular}{|ll||c|c|c:c|}
\hline & $/ \mathrm{mo}_{1} \cdot \mathrm{se}_{2}$ Incompl. $_{\text {In }}$ & $\mathrm{RM}$ & $\mathrm{Max}$ & Lin & Unif \\
\hline \hline a. & mo.se & $* !$ & & & \\
\hline b. & $\mathrm{mö}_{1,2} \mathrm{~S}$ & & & $*$ & $*$ \\
\hline c. & $\mathrm{mo}_{1} \mathrm{~s}$ & & $* !$ & & \\
\hline
\end{tabular}

The umlaut process certainly violates Linearity and Uniformity in Rotuman, but they are not the only constraint violations of relevance. Mainly two vocalic feature identity constraints are involved additionally: vowel height and vowel backness. The relevant feature identity constraints should be ranked beneath Max to keep [mös] as the winner in (36). One may bring up the input-output correspondence as depicted in (37), where umlaut still comes from the final vowel but the final vowel 
remains without undergoing fusion. Since this form violates neither Linearity nor Uniformity, it is a serious competitor. My claim is that this candidate is ruled out by Integrity, another faithfulness constraint never violated in the incomplete phase formation. A new aspect is that the domain where Integrity is active is not restricted to segments but is expanded to the featural level.

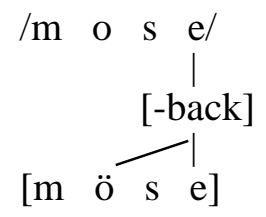

Considering final vowel deletion in (27d), a tableau is given in (38), where some constraints in (32) and stress information are included since they are of direct relevance. Stressed syllables are underscored. As (38) shows, any attempt to satisfy RM in a way other than vowel deletion infringes on some undominated constraint, suggesting that subtraction is a highly expensive strategy: subtraction is the best only when all other options result in a violation of some undominated constraint.

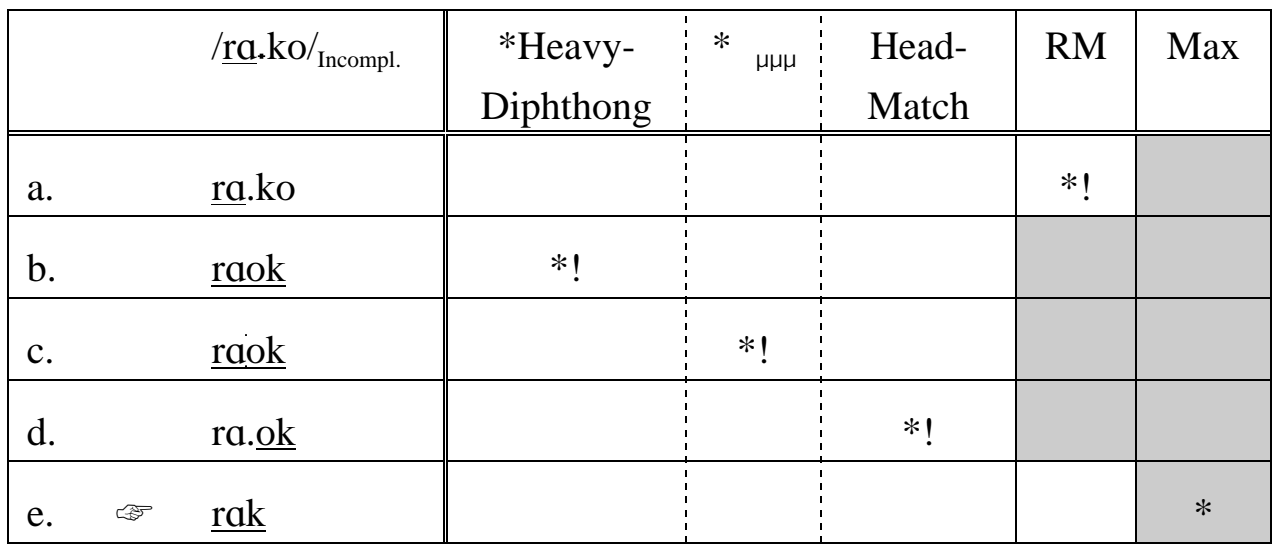

But the incomplete phase formation occasionally pays more, reaching the maximal extreme. The relevant examples are in (27e). When a complete phase form 
ends in a long vowel, no overt exponent of the incomplete phase surfaces. (39) represents some potential candidates for the input /si.kad/. As the tableau indicates, no stem modification can avoid the violation of some kind of undominated constraint ranked over RM. The upshot is to give up on any overt phonological expression of the incomplete phase morpheme in cases as in (27e).

\begin{tabular}{|ll||c|c|c|c|}
\hline & \multicolumn{1}{|c||}{$/$ si.kad/Incompl. } & Weight-Ident & $* \sigma_{\mu \mu \mu}$ & Head-Match & RM \\
\hline a. & si.(kaa) & & & & $*$ \\
\hline b. & $($ si.ka $)$ & $* !$ & & & \\
\hline c. & si.(aak) & & $* !$ & & \\
\hline d. & $($ saa).ki & & & $* !$ & \\
\hline
\end{tabular}

Under the present analysis where RM plays a prominent role as the driving force of nonconcatenative base modifications, the reason why the examples in (27e) do not undergo any changes is due to the pressure of the undominated constraints. This accords with the observation made in the nominative formation in Lardil that an explicit morphemic realization is prevented when any attempt results in a violation of some phonological constraint ranked higher than RM.

\subsubsection{McCarthy $(2000 c)$}

The main concern of McCarthy (2000c) is to expand the domain of faithfulness constraints to prosodic information, so it does not share the same principal interest as the goal of this chapter. Despite this, it provides a comprehensive OT analysis of the phase alternation at issue. The distinction of prosodic structure between the two phases discussed in the preceding section plays a direct role in McCarthy's (2000c) 
analysis. He claims that the incomplete phase formation is a phonological effect, which makes the word-final portion be occupied by a stressed bimoraic monosyllable, paying attention to the invariance of the word-final prosodic structure of the incomplete phase. Various nonconcatenative allomorphs are needed to achieve this goal (although "allomorphs" is not an appropriate terminology in the context of his analysis). McCarthy (2000c:160) directly captures the prosodic contrast through the phonological markedness constraint in (40).

Align-Head- $\sigma$ : Align $\left(\mathrm{H}^{\prime}(\operatorname{PrWd}), \mathrm{R}, \operatorname{PrWd}, \mathrm{R}\right)$

The main-stressed syllable is final in every word.

The only possible syllables are $(\mathrm{C}) \mathrm{V}$ in Rotuman generally except in the word-final position of the incomplete phase or in words with long vowels, which are also found only word-finally. According to McCarthy, the reason why the incomplete phase deviates from the very rigid syllable structure constraint is that Align-Head- $\sigma$ outranks Syll $=\mu$ (a constellation of constraints responsible for deriving only (C) $\mathrm{V}$ syllables). Under McCarthy's analysis, the examples in (27e) are unremarkable. Since the complete phase forms already satisfy Align-Head- $\sigma$, no ado is required for the incomplete phase formation. Any attempt to satisfy the constraint by adding some modification to the complete phase base results in meaningless faithfulness violations, so complete faithfulness is the most harmonic. The central constraint ranking posited by McCarthy (2000c) is given in (41).

Align-Head- $\sigma$ » Max » Linearity, Uniformity

This is quite similar to the ranking proposed in (29), the only difference being which constraint operates as the driving force of base modifications. He does not 
discuss the diphthongization process in (27a) in detail, but it can be safely assumed that the constraint militating against it is ranked below Linearity and Uniformity. Given this constraint ranking, deletion still has the most severely restricted distribution while diphthongization is the least expensive stem modification among the range of nonconcatenative allomorphs employed in the incomplete phase formation. The more stringently restricted, the higher markedness constraints must be satisfied.

The significant difference between the two analyses lies in the driving force of the various stem modifications in the incomplete phase. While my analysis employs morphology-oriented RM, a phonological markedness constraint is used in McCarthy's analysis. His basis of the phonological analysis comes from the argument developed by Hale and Kissock (1998). Examining Churchward's (1940) descriptive documentation, they claim that the phase alternation is phonological rather than syntactic or semantic. They observe that the two phases are associated with complementary suffixes and clitics. Crucially, complete phase forms are linked only to monomoraic suffixes and clitics whereas the incomplete phase occurs when a suffix is bimoraic or larger or when no suffixal element follows a stem, as shown in (42) (Hale and Kissock 1998:120).

(42) The complete phase

$\begin{array}{ll}\text { - „a } & \text { 'nominalizer' } \\ \text {-me } & \text { 'hither' } \\ \text {-(a)fu } & \text { 'away (towards listner)' } \\ \text {-(a)Đe } & \text { 'away (towards third person)' } \\ \text {-a } & \text { 'completive aspect' } \\ \text {-a } & \text { 'transitive' } \\ \text {-e } & \text { 'locative anaphor' } \\ \text {-t } & \text { 'indefinite singular article' } \\ \text {-s } & \text { 'interrogative' } \\ \text { - } \varnothing & \text { 'definite plural/locative' }\end{array}$

The incomplete phase

$\begin{array}{ll}\text { - Tia } & \text { 'ingressive' } \\ \text {-tia } & \text { 'completive aspect' } \\ \text {-?aki } & \text { 'causative' } \\ \text {-kia } & \text { 'transitive' } \\ \text {-?ian } & \text { 'ingressive' } \\ \text {-ta?a } & \text { 'that' } \\ \text {-tei } & \text { 'vocative particle' } \\ \text {-tema } & \text { 'each' } \\ \text {-te?isi } & \text { 'this' } \\ \text {-ta } & \text { 'definite singular article' }\end{array}$


In (42), there are five apparent exceptions to the generalization above: $-t$ (indefinite singular article), $-s$ (interrogative), two instances of $-\varnothing$ (definite plural/locative) and - $t a$ (definite singular article). The first four are unexpected since they are submoraic despite the fact that they are employed in the complete phase. The final one is not expected because it is monomoraic although it is used in the incomplete phase. Hale and Kissock argue that they are actually not exceptional. For the present purposes, I review their discussion of the indefinite singular article $-t$ and the definite singular article $-t a$.

They crucially assume that an indefinite article and the lexeme meaning one have a close connection. They claim that the lexeme meaning one in Rotuman is $t a$ (although this is factually wrong) and that the indefinite singular article is /-ta/ in the underlying representation (e.g., /vaka-ta/ 'the canoes'). The immediate question is then how to distinguish the indefinite and the definite singular articles since they both exhibit the same form -ta (e.g., /vaka-ta/ 'the canoes/a canoe') at the underlying level. The proposed answer to this puzzle is to decompose the definite singular morpheme into two distinct morphemes: definite and singular morphemes where the definite morpheme contains a phonetically unpronounced mora (e.g., /vaka-ta $\operatorname{singular}^{-} \emptyset_{\text {definite }} /$ ). In McCarthy's (2000c:162) terms who fully adopts Hale and Kissock (1998), the definite article consists of a floating mora. Given this, the definite singular morpheme in (42) is bimoraic, and therefore, the surface -ta conforms to the descriptive generalization that all suffixes and clitics used in the incomplete phase are more than monomoraic, effectively distinguishing the definite and the indefinite singular morphemes. Furthermore, the indefinite singular morpheme is reanalyzed as a monomoraic morpheme, again obeying Hale and Kissock's generalization. In 
effect, Hale and Kissock argue that the five exceptions are simply superficial since they all receive a similar line of reanalysis. Indeed, Churchward (1940:14) states that there are no suffixes which can be attached indiscriminately to either phase. Moreover, the syntactico-semantic environments where the two phases are used are admittedly unclear. Therefore, Hale and Kissock's idea to explain the phase alternation in phonological terms is interesting.

Based on Hale and Kissock's claim, McCarthy claims that the alignment constraint in (40) captures the complete-incomplete phase distinction. He assumes that suffixes larger than a mora have a foot status and stand as prosodic words on their own independently of the preceding stem, as illustrated in (43) (McCarthy 2000c:163). Given this representation, a prosodic word boundary coincides with the right edge of the stem, and therefore, /... CVCV/ undergoes some stem modification such as metathesis and fusion to create [...CVC]. The final CVC syllable carries the main stress and is properly aligned to the right edge of the prosodic word dominating the stem. This explains why various stem modifications take place in the incomplete phase because the second last syllable of the stem would carry main stress otherwise due to RHType $=$ Trochaic.

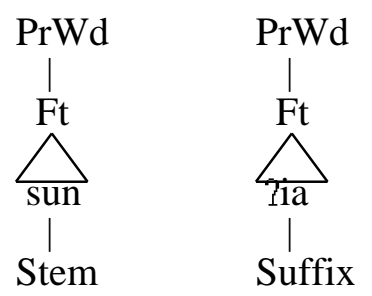

By contrast, monomoraic suffixes do not constitute a foot, and therefore, they do not stand as independent prosodic words. As a consequence, they are prosodically dependent on the prosodic word dominating a stem, as shown in (44). This is a 
reconstructed underlying representation on the basis of McCarthy's textual explanation since the structure of the complete phase given by McCarthy (2000c:163) is inconsistent with his discussion. In this structure, a stem is not a free-standing prosodic word since the following suffix is also part of the same prosodic word. The alignment constraint in (40) is irrelevant to determining the stem form itself since it is not coextensive with the prosodic word. Since the alignment constraint is sensitive to the prosodic word, the entire form including the suffix attached to the stem must obey the alignment restriction. As in [fere-ay] 'flying' (Churchward 1940:22), therefore, the suffix undergoes some phonological modification in the complete phase.

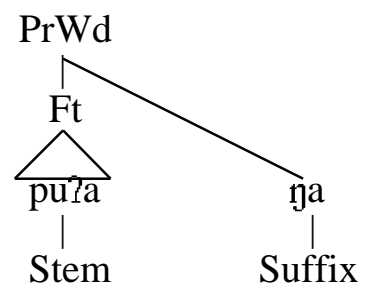

Although McCarthy's analysis is consistent with the phonological generalization made by Hale and Kissock (1998), their argument, on which McCarthy's analysis is based, has a number of problems. First of all, their initial assumption is not supported. Churchward (1940:15 fn.4) explicitly mentions that "this [the definite singular article] -ta must not be confused with taa, meaning one, in which the $a$ is long..." (see also Churchward 1940:36). Long vowels are clearly distinguished from short vowels by attaching a macron over long vowels in Churchward's documentation. This indicates that the indefinite singular morpheme and the lexeme representing one are clearly distinguished phonologically, so Hale and Kissock's morphological reanalysis is dubious from the beginning. Even if their morphological reanalysis is adopted, the result should be -taa rather than -ta. But 
given this reanalysis of the indefinite singular morpheme, the motivation to decompose the definite singular morpheme into two components is lost since the definite and the indefinite singular morphemes are phonologically distinguishable. This suggests the invalidity of the reanalysis implemented by Hale and Kissock. This in turn shows that the indefinite singular morpheme is not an apparent but real exception to their phonological distinction of the two phases.

Second, it is not clear why the vowel of /-ta/ is lost in the indefinite singular morpheme when realized in the surface representation if their reanalysis were on the right track. Rotuman is basically a CV language. Therefore, there is no phonological reason to prohibit a word-final vowel. This suspicion is natural especially because the indefinite singular article is used in the complete phase, and as discussed in section 4.5.2, the complete phase admits only $(\mathrm{C}) \mathrm{V}$ syllables modulo long vowels in the word-final position is the only exception. Hale and Kissock do not provide any explanation for this question, but it should be morphologically governed. Whatever the reasoning is, however, the vowel must be always subject to deletion. The obvious question is why a morpheme contains a vowel which never surfaces. Since the vowel presence and absence never exhibit an alternation, the analysis stipulates a phonologically unmotivated abstract vowel. Furthermore, it is also unclear why the floating mora of the definite article posited in the reanalysis is not realized in the form of lengthening of a vowel affiliated with the singular morpheme, for instance (e.g., [vaka-taa] where the suffix -taa is the simultaneous exponent of the definite and the singular morphemes). The argument here shows that neither the vowel in the indefinite singular morpheme nor the floating mora posited as a result of Hale and Kissock's morphological reanalysis receives strong phonological underpinning. In 
other words, they posit several unmotivated abstract phonological units. These points cast a doubt that the phonological explanation for the phase alternation is a castle in the air. This is problematic for McCarthy's (2000c) phonological analysis as well. Given that the alignment constraint in (40) is a pure markedness constraint, it is insensitive to the underlying representation. Unless any of the abstract vowels posited by Hale and Kissock surfaces, the stem followed by the definite singular article /-ta/ would be expected to take a complete phase form, contrary to fact.

Third, Churchward (1940:15) provides several minimal pairs where only different stem forms denote different functions, as cited in (45). In each pair, the first one is the complete phase, the second one being the incomplete phase. The words exhibiting the stem alternation are underscored. Since no suffix or clitics is involved in these examples, a purely phonological analysis of the phase alternation has no explanation for the fact that the complete and incomplete phases take different stem shapes. Churchward (1940:15) states that the definite-indefinite distinction is often made explicit by attaching /-ta/ or /-t/, but crucially, these suffixes are not obligatory. This shows that Hale and Kissock's generalization that only the incomplete phase is employed when a stem is not followed by any suffix or clitics is falsified.
a. famori ?ea
'The people say.'
famör ?ea
'Some people say.'
b. $\quad$ ?epa la hoa?
'The mats will be taken.'
'Some mats will be taken.'
c. Täe la ?oaf se mori
Täe la ?oaf se mör
'Would you like the oranges?'
'Would you like some oranges?'
d. gou ho?am ?eu kalofi 'I have brought your eggs (the eggs belonging to, or intended for, you).' gou ho?am ?eu kalöf 'I have brought you some eggs.' 
The review above strongly shows that a purely phonological analysis of the phase alternation cannot be maintained. Hale and Kissock (1998) correctly point out that the precise syntactic-semantic contexts for each phase are not clearly understood. But examples as in (45) are never expected under a phonological explanation of the complete-incomplete phase distinction. McCarthy's (2000c) phonological analysis crucially relies on Hale and Kissock (1998) and does not give any independent argument for a phonological analysis. The alignment constraint in (40) is a pure phonological markedness constraint, and therefore, no morphological factor is taken into account for the evaluation of its satisfaction or violation. Given that it is the only constraint in McCarthy's analysis which drives the incomplete phase formation, I conclude that his analysis is not supported.

One might point out as a potential problem of my analysis that the modified portion does not follow from any constraints. Word-final portions are always affected, but how can we explain this restriction without (40)? Many cases are indeed dealt with by the generalization and analysis presented above without any further ado. For example, the reason why the word-initial CV cannot be metathesized can be reduced to the undominated constraints in (32). Consider/pu.re/ as the input and an output candidate [up.re]. The rhythmic type constraint and the alignment constraint in (32) requires stress to fall on the initial syllable in the output, but in [up.re], stress assignment on the same syllable violates the alignment constraint. If the final syllable carries stress, Head-Match is violated. In the same vein, many other potential possibilities are successfully eliminated by the analysis presented above.

To ensure that only the word-final part of the complete phase is altered, however, we can posit a positional faithfulness constraint such as Faith-IO- $\sigma 1$ 
(Beckman 1995, 1997). Comparing the word-internal and word-final positions, affecting the word-medial part incurs more massive violations of Contiguity than changing the word-final portion. Given that these two constraints are presumably needed for independent reasons, the absolute generalization that only the word-final portion is affected in the phase alternation follows without specifying the prosodic shape of the incomplete phase. The fact that the incomplete phase formation creates an otherwise entirely impermissible syllable structure is captured by ranking Syll $=\mu$ below RM such that Rotuman allows for more room to realize the incomplete phase morpheme.

\subsection{Summary}

In this chapter, I investigated how nonconcatenative allomorphs can be explained in a principled manner. It is clear from the brief review of various earlier studies that nonconcatenative allomorphs have not received a unified understanding so far. I claimed that the reason why various base modifications are employed to express a single morpheme is to optimize the word form as much as possible, achieving phonologically less marked structures. In this sense, nonconcatenative allomorphs are nothing different from concatenative allomorphs such as the allomorphy displayed in the alternation of the plural suffix $-s$ in English. This is not surprising given the claim that concatenative morphology and nonconcatenative morphology should be understood in a unified way despite the terminological distinction established in the discipline. The actual aspect formation in Saanich provides particularly strong evidence for this integration since both types of morphology coexist as allomorphs of the actual aspect morpheme. No theory to tease them apart would succeed in 
providing a satisfactory account of such cases. RM plays a role as the key constraint, and interactions of various constraints ranked below it determine what kind of base change is the most harmonic. Although violating the faithfulness constraint ranked the lowest is the cheapest strategy to realize a morpheme, some other nonconcatenative allomorph surfaces when the violation of the lowest faithfulness constraint results in a concomitant violation of a high ranked markedness constraint.

Several attempts have been made to account for multiple nonconcatenative allomorphs, but I argued that RMT overcomes various problems encountered by them. Moreover, the empirical issues discussed in this chapter clearly distinguish RMT and anti-faithfulness theory. As argued in section 4.3.2, anti-faithfulness theory cannot deal with multiple nonconcatenative allomorphs essentially because nothing in the theory guarantees that multiple anti-faithfulness constraints are not in conflict with crucial markedness constraints or that anti-faithfulness constraints ranked over faithfulness counterparts are all in conflict with markedness constraints. Thus, complementary distribution of nonconcatenative allomorphs presents a serious challenge to anti-faithfulness theory. Empirical data covered in this chapter are also in favor of RMT over anti-faithfulness theory. 


\section{Chapter 5}

\section{Double Morphemic Exponence as Morphological Opacity}

\subsection{Introduction}

This chapter addresses issues surrounding what is here called double morphemic exponence (DME). DME is exemplified in (1) from the plural formation in German, where examples are given orthographically (see Zwicky 1967; Bach and King 1970; Robinson 1975; Strauss 1976; Lieber 1981, 1992; Janda 1982ab; Lodge 1989; Wiese 1996ab among many others). As amply discussed so far, a morphological expression exists as long as some phonological exponent of a morpheme surfaces. The plural formation in (1) is peculiar in this light because the plural forms are different from the singular counterparts in two respects: the presence of a suffix and umlaut on a stem vowel. Given that RM is satisfied by the presence of the suffix alone, the immediate question is why this kind of redundant phonological exponence is observed in natural languages.

Singular Plural Gloss

$\begin{array}{llll}\text { a. } & \text { Gast } & \text { Gäste } & \text { guest(s) } \\ & \text { Gaul } & \text { Gäule } & \text { pack horse(s) } \\ & \text { Nacht } & \text { Nächte } & \text { night(s) } \\ \text { Kraft } & \text { Kräfte } & \text { power } \\ & \text { Not } & \text { Nöte } & \text { pain(s) } \\ & & & \\ \text { b. } & \text { Buch } & \text { Bücher } & \text { book(s) } \\ & \text { Mann } & \text { Männer } & \text { man/men } \\ & \text { Dach } & \text { Dächer } & \operatorname{roof}(\mathrm{s})\end{array}$

A conceivable but immediately rejected hypothesis is recourse to a phonological reason: to achieve less marked structure. This is incorrect because there is no plausible phonological reason behind the umlaut process. Indeed, umlaut 
creates a more marked vowel given the crosslinguistic tendency that front vowels are unrounded by default.

Umlaut is thus morphological, but why is it required? Various derivational analyses maintain different positions regarding their specific analytical implementations, but the focus of discussion has centered on how the distribution of umlaut is to be accounted for in a principled way. But they do not attempt to explain why DME as in German plurals takes place in the first place. As discussed and analyzed later in this chapter, DME is by no means limited to the plural formation in German. DME is frequently observed in various languages although it is not as pervasive as single morphemic exponence across languages. The formal mechanism underlying in DME is therefore an empirical issue which must receive a satisfactory understanding.

The remainder of this chapter is organized as follows. Section 5.2 outlines my general proposal to answer why DME is required and how it should be explained theoretically in the overall context of this work. The motivation and mechanism of DME are closely wired with each other, so they should be explained in a unified manner. I propose the idea of morphological opacity in the sense to be clarified and fleshed out later. Furthermore, I argue that sympathy theory (McCarthy 1999) crystallizes morphological opacity in DME from a formal theoretic perspective. The following three sections are devoted to case studies. Section 5.3 deals with so-called dominant affix effects in Japanese, where attachment of a certain suffix causes accent deletion, preaccentuation, or accent shift, depending upon the nature of the suffix. In section 5.4, I discuss the impersonal formation in Chaha. What is interesting here is that morphologically conditioned palatalization and labialization take place 
simultaneously. Contrary to various analyses developed in the earlier literature, I argue that this morphological process should be subsumed under the rubric of DME. In section 5.5, I present interactions of affixation and reduplication in Tagalog, which exhibit DME fully integrated in the language. The proposal made in section 5.2 is applied to phonological polarity in section 5.6. Phonological polarity is an important empirical domain that Alderete (1999) discusses to motivate anti-faithfulness theory. Discussing the singular $\approx$ plural alternation in Luo, I argue that the general proposal for DME succeeds in accommodating phonological polarity. In section 5.7, I return to the proposal made in section 5.2 and discuss its theoretical predictions. The discussion is primarily concerned with the formal restrictiveness of the proposal and predictions regarding possible and impossible morphological constructions in natural languages. I also discuss that anti-faithfulness theory makes different predictions in this context. Finally, section 5.8 briefly summarizes the main results of this chapter.

\subsection{Morphological Opacity and Sympathy Theory}

This section is aimed at providing a principled and integrated answer to the two questions raised above: (i) why DME takes place, and (ii) how it should be formally analyzed. Given the fact that many morphemes are phonologically expressed either by adding an affix (e.g., English plurals) or by simply modifying the phonological shape of the base (as in a lot of nonconcatenative morphological processes discussed so far), it is at first glance a mystery that both affixation and a phonological change of a base should be necessary at the same time for a single morphemic expression.

The reason why no stem modification is necessary in most cases of affixation is that it is sufficient for the satisfaction of RM. On the other hand, some kind of 
stem modification is employed when no affix exists underlyingly because it is the only eligible strategy to avoid a violation of RM. This apparently trivial observation is indeed the key toward a satisfactory understanding of DME. Building on this, I propose that DME occurs when the affix is made invisible to RM through some mechanism to be fleshed out shortly. Consider the illustration given in (2) to illuminate this idea. The entire word contains a suffix as well as a stem, but the suffix is invisible, hence morphological opacity. The suffix behaves as if it were not present in the structure, making only the stem available as a visible element. For the aforementioned reasons, the stem needs some phonological change due to the pressure of RM: the cooccurrence of affixation and a stem modification makes sense if the affix is made opaque for the purpose of satisfying RM.

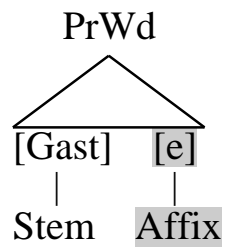

My proposal is that the effect of morphological opacity is obtained through sympathy theory, originally advanced by McCarthy (1999) to handle phonological opacity (Kiparsky 1971, 1973). Consider (2) as an example. What is needed here is to make the suffix invisible. This is in effect tantamount to articulating a system to exclude the suffix from the word domain. This is achieved by assuming Stem $\equiv P r W d$ as the selector constraint. The effect of this constraint is to make the domain of a stem accord with that of a prosodic word. As schematically illustrated in (3), the output form that satisfies this constraint has a structure in which the suffix is disregarded from the prosodic word domain. In other words, the stem domain is 
coextensive with the prosodic word domain. Since RM is sensitive to a prosodic word, this output representation violates RM as it stands. Thus, (3) is precisely the configuration where some nonconcatenative morphological operation takes place, the only difference being whether an opaque affix is present or not. The structure in (3) thus requires a phonological change on the stem, the specific instantiation of which depends upon which Faith-IO constraint is ranked lower than RM.

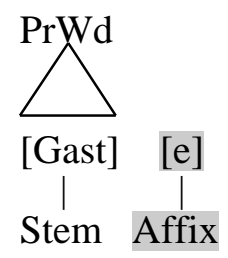

An important issue is the possibility that the entire word encompassing the stem and the suffix has the recursive prosodic word structure as in (4). In this case, the stem is coextensive with $\operatorname{PrWd}_{2}$, but $\operatorname{PrWd}_{1}$ subsumes both the stem and the suffix. Although this word structure is a possibility, this does not impinge upon the argument. Assuming that $\mathrm{Stem} \equiv \mathrm{PrWd}$ is operative with respect to all prosodic word nodes contained in the representation, one violation is incurred because the stem domain does not coincide with the $\operatorname{PrWd}_{1}$ domain. This indicates that the recursive prosodic word structure of the kind depicted in (4) fares worse than the structure in (3), everything else being equal.

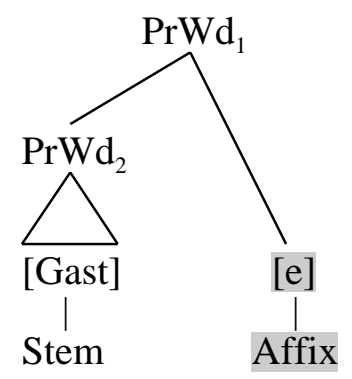


Returning to the German plural examples in (1), the analysis is given in (5), where Stem $\equiv$ PrWd plays a key role as the selector constraint. As far as Stem $\equiv$ PrWd is concerned, output candidates yielded by Gen are all required to have the structure in (3). (5c) is more harmonic between the two candidates fulfilling Stem $\equiv$ PrWd. Since this is the selector constraint, $(5 \mathrm{c})$ is opted for as the sympathy candidate due to the fact that Ident-IO-[+back] is ranked lower than RM. (5b) and (5d) are immediately eliminated from the selection of the sympathy candidate because they violate the selector constraint itself. But a further step is needed: the well-formedness evaluation of overall candidates with respect to the sympathy candidate through Ident- $\mathrm{O}$-[back]. (5d) satisfies this constraint, but (5a) and (5b) do not. Especially, (5b) incurs a fatal violation here because it satisfies RM by virtue of the fact that it faithfully parses the suffix associated with the plural morpheme. Candidates (5a) and (5c) are ruled out by high ranking Max. Thus, the intuition behind this sympathy analysis is pretty straightforward: faithful parsing of the suffix is required by high ranked Max, but a stem modification is added since the suffix behaves as if it were not there. Stem $\equiv \mathrm{PrWd}$ and Ident- ${ }^{2} \mathrm{O}-[\mathrm{back}]$ jointly require the optimal form to agree with the sympathy candidate in the relevant vowel quality change. Certain morphemes activate ${ }_{3}$ Stem $\equiv \mathrm{PrWd}$ when they receive two phonological exponents.

\begin{tabular}{|c|c|c|c|c|c|c|}
\hline & /Gast-e/ ${ }_{\text {Plural }}$ & Max & RM & $\begin{array}{c}\text { Ident-s } \\
\text { [back] }\end{array}$ & $\begin{array}{r}\text { Ident-IO } \\
\text { [+back] }\end{array}$ & $\begin{array}{l}\text { S Stem } \\
\equiv \text { PrWd }\end{array}$ \\
\hline a. & Gast & $* !$ & $*$ & $*$ & & \\
\hline b. & Gaste & & & $* !$ & & $*$ \\
\hline c. & Gäst & $* !$ & & & $*$ & \\
\hline d. & Gäste & & & & $*$ & $*$ \\
\hline
\end{tabular}


Building on this argument, I propose a generalized schema of DME. Several crucial ranking relations can be excerpted from (5). First, it is essential that RM outrank Ident-IO-[+back] so that a candidate with umlaut is selected as the sympathy candidate (compare (5a) and (5c)). Second, Ident- $\mathrm{O}$-[back] needs to be ranked over Ident-IO-[+back] because the sympathy candidate would not exercise any influence over other candidates otherwise (compare (5b) and (5d)). Finally, the fact that the optimal form carries the underlying suffixal element $-e$ suggests that Max dominates Stem $\equiv \operatorname{PrWd}$ (compare (5c) and (5d)). Given these crucial rankings, the generalized schema of DME looks as in (6). The reason why umlaut takes place in the plural formation in German is that relevant faithfulness constraints are Ident-IO-[+back] and Ident- 8 -[back]. Faith-IO and Faith- 80 are variables, so various nonconcatenative morphological operations can be obtained depending on the specific faithfulness constraints replacing these variables.

General ranking schema of DME

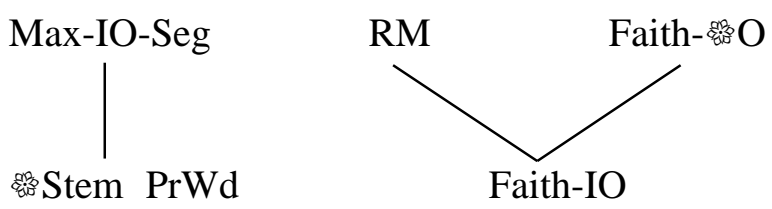

This general ranking schema is demonstrated below. Consider the following schematic example: /ABC- $\mathrm{D}_{\alpha} / \alpha \rightarrow[\mathrm{ABC} \mathrm{D}]_{\alpha}$, where the three segments $A B C$ belong to a stem and the remaining one $D$ to a suffix, $/ \mathrm{C} / \rightarrow\left[\mathrm{C}^{\prime}\right]$ represents a nonconcatenative stem change, and $\alpha$ is an arbitrary morphosyntactic category distinct from that expressed by $[\mathrm{ABC}]$. As illustrated in (7), the generalized schema in (6) successfully generates $[\mathrm{ABC} \mathrm{D}]_{\alpha}$. 


\begin{tabular}{|c|c|c|c|c|c|c|}
\hline & $/ \mathrm{ABC}-\mathrm{D}_{\alpha} /_{\alpha}$ & Max & $\mathrm{RM}$ & $\begin{array}{c}\text { Faith } \\
\text { O-[C'] }\end{array}$ & $\begin{array}{c}\text { Faith } \\
\text { IO-[C] }\end{array}$ & $\begin{array}{l}\text { S Stem } \\
\equiv \text { PrWd }\end{array}$ \\
\hline a. & $\mathrm{ABC}$ & *! & $*$ & $*$ & & \\
\hline b. & ABCD & & & $* !$ & & $*$ \\
\hline c. 8 & $\mathrm{ABC}^{\prime}$ & $* !$ & & & $*$ & \\
\hline d. & ABC'D & & & & $*$ & $*$ \\
\hline
\end{tabular}

The selector constraint (i.e., 8 Stem $\equiv P r W d$ ) might seem to be excessively general for explaining the plural formation data of German, but this is empirically motivated. The reason is that suffixation is not the only affixation involved in DME. Languages actually exhibit DME in the context of prefixation, circumfixation, and infixation as well, each of which will be exemplified shortly. As in (8), the stem and the prosodic word must be completely coextensive for the sympathy system to function since the affixal portion can be regarded as the phonological exponent of the relevant morpheme. It is thus indispensable to establish a selector constraint that is applicable to any kind of affixation. The two affix $x_{\alpha} \mathrm{s}$ in circumfixation and $\mathrm{stem}_{\alpha} \mathrm{s}$ in infixation are intended to indicate that they are a single affix and a stem respectively.

$$
\begin{aligned}
& \begin{array}{lll}
\text { Prefixation } & \text { Circumfixation }
\end{array} \\
& \frac{\text { PrWd }}{[\text { Affix }][\text { Stem }]} \quad \frac{\text { PrWd }}{\left[\text { Affix }_{\alpha}[\text { Stem }] \text { Affix }_{\alpha}\right]} \quad \frac{\text { PrWd }}{\left[\text { Stem }_{\alpha}[\text { Affix }] \text { Stem }_{\alpha}\right]}
\end{aligned}
$$

Stem $\equiv \operatorname{PrWd}$ is more formally understood in terms of the conjunction of the constraints given in (9). First, Anchor-R(Stem,PrWd) requires that the segment occupying the right edge of the stem must be positioned at the right edge of a prosodic word. This constraint thus has the effect to exclude a suffix from the domain of a prosodic word, as in (3). Second, the mirror image holds of Anchor- 
$\mathrm{L}(\mathrm{Stem}, \mathrm{PrWd})$, and therefore, a prefix is disregarded for the purpose of computing the element within the prosodic word domain. Third, in infixation, Contiguity is violated by virtue of the fact that the phonological string of a stem is separated by the affixal element. This indicates that Contiguity-Stem is the relevant constraint to exclude the infix from the domain of a prosodic word. Finally, circumfixation is the combination of prefixation and suffixation, and therefore, the effect of Stem $\equiv \operatorname{PrWd}$ is obtained through conjunction of Anchor-L(Stem,PrWd) and Anchor-R(Stem,PrWd) operative in prefixation and suffixation. This yields the effect of Hierarchical Anchor in the sense of Itô, Kitagawa and Mester (1996).

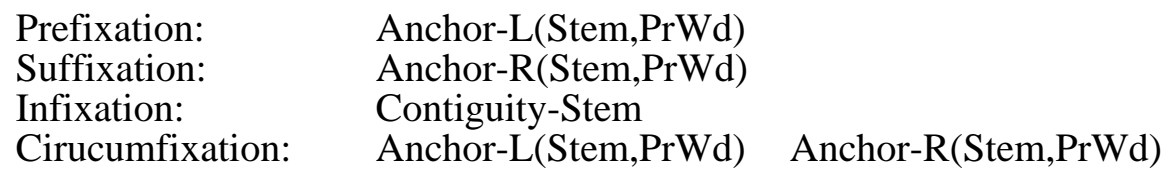

Crucial here is that the proposed constraint conjunction is propositional logical conjunction (Hewitt and Crowhurst 1996; Crowhurst and Hewitt 1997; Itô and Mester 1999a), where the conjoined constraint is satisfied only when all conjoined constraints are individually satisfied. It is violated everywhere else. This positive conjunction (in Crowhurst and Hewitt's 1997 terminology) or best-of-the-best (in Itô and Mester's 1999a terminology) is shown in the evaluation table in (10a). Since three constraints are conjoined here, $\mathrm{Stem} \equiv \mathrm{PrWd}$ is satisfied only when all of them are satisfied simultaneously. This sharply contrasts with negative/disjunctive conjunction (in Crowhurst and Hewitt's 1997 terminology) or worst-of-the-worst (in Itô and Mester's 1999a terminology), where a conjoined constraint is violated if and only if both conjuncts are violated (Smolensky 1993, 1995, 1997), as in (10b). The crucial difference appears when only one of the conjoined constraints is violated. The whole conjoined constraint is violated in positive conjunction when only one conjunct 
is satisfied. By contrast, the entire constraint is satisfied in such cases under negative conjunction.

(10) a. Positive conjunction (Best-of-the-best)

\begin{tabular}{|c||c||c|}
\hline Constraint $_{1}$ & $\wedge$ & Constraint $_{2}$ \\
\hline \hline Satisfied & Satisfied & Satisfied \\
\hline Satisfied & Violated & Violated \\
\hline Violated & Violated & Satisfied \\
\hline Violated & Violated & Violated \\
\hline
\end{tabular}

b. Negative/disjunctive conjunction (Worst-of-the-worst)

\begin{tabular}{|c||c||c|}
\hline Constraint $_{1}$ & $\& / \vee$ & Constraint $_{2}$ \\
\hline \hline Satisfied & Satisfied & Satisfied \\
\hline Satisfied & Satisfied & Violated \\
\hline Violated & Satisfied & Satisfied \\
\hline Violated & Violated & Violated \\
\hline
\end{tabular}

Establishing the formal aspect of Stem $\equiv \operatorname{PrWd}$, I present cases where DME is involved in affixation other than suffixation. First, Tagalog presents a good representative example of DME involved with prefixation. In this language, prefixation and reduplication cooccur quite regularly and productively to stand for a single morphosyntactic function (Carrier 1979, 1984; Lieber 1981; McCarthy 1981; Marantz 1982; French 1988). This is exemplified in (11), where trabahoh is a stem meaning 'work' and mag-/nag- is an agentive prefix. The crucial observation is that the same prefix form is employed in more than one morphosyntactic category (i.e., mag- is used in both the basic and future aspects, and nag- is employed both in the 
completive and continuative aspects). Furthermore, the same reduplicant shape is recycled to express various morphosyntactic categories (i.e., $\mathrm{CV}$-reduplication is observed in both the future and continuative aspects). This strongly suggests that prefixation and reduplication are both indispensable to disambiguate various morphosyntactic categories (Lieber 1981:158-159): just one of them is not sufficient to specify a particular morphosyntactic function.

\section{(11)}

\section{Affixed/reduplicated form Gloss}

$\begin{array}{ll}\text { mag-trabahoh } & \text { work (basic aspect) } \\ \text { mag-ta-trabahoh } & \text { will work (future aspect) } \\ \text { nag-trabahoh } & \text { worked (completive aspect) } \\ \text { nag-ta-trabahoh } & \text { be working (continuative aspect) }\end{array}$

Under the system developed here, the future aspect formation, for instance, is explained simply by replacing Faith-IO and Faith- ${ }_{80} \mathrm{O}$ with Integrity-IO and Max-8 respectively, as shown in (12). In (12), the precise size restriction on the reduplicant is not of immediate relevance, and hence, I simply assume here that it obtains through the emergence of the unmarked (McCarthy and Prince 1994), as argued by Spaelti (1997), Walker (1998), and McCarthy and Prince (1999) among others. I discuss this issue in more detail in section 5.5.2.

\begin{tabular}{|c|c|c|c|c|c|c|}
\hline & /mag-trabahoh/Future & $\operatorname{Max}$ & $\mathrm{RM}$ & $\begin{array}{c}\mathrm{Max} \\
\mathrm{O} \\
\end{array}$ & $\begin{array}{c}\text { Integrity } \\
\text { IO }\end{array}$ & $\begin{array}{l}\text { Sistem } \\
\equiv \text { PrWd }\end{array}$ \\
\hline a. & trabahoh & $* ! * *$ & $*$ & $* *$ & & \\
\hline b. & mag-trabahoh & & & $* ! *$ & & $*$ \\
\hline c. & ta-trabahoh & $* ! * *$ & & & $* *$ & \\
\hline d. $\quad$ खि & mag-ta-trabahoh & & & & $* *$ & * \\
\hline
\end{tabular}

Several remarks are in order regarding reduplication in the context of DME. Given the fact that the reduplicant blocks the underlying stem (or base) from being 
anchored at the left edge of the prosodic word, it might appear that (12c) violates Stem $\equiv$ PrWd. This is a significant issue since reduplication would not be motivated at all if this were true. My contention is that Stem $=\operatorname{PrWd}$ is indeed satisfied by the sympathy candidate. Under the reduplication model assumed here (see section 2.2), the source of the reduplicant is the same as the base since the entire reduplication form is evaluated by IO-faithfulness constraints. This indicates that the leftmost segment of the underlying stem still occupies the left edge of the whole reduplication form even though the base-initial segment is not left-anchored to the prosodic word. I claim that Anchor-L(Stem,PrWd) is satisfied if there exists some element at the left periphery of the output which is the correspondent of the leftmost element in the underlying representation. As a consequence, Stem $\equiv \operatorname{PrWd}$ is also satisfied by (12c). Again, each underlying segment has two opportunities to be realized on the surface (i.e., base and reduplicant) under the assumed reduplication model, so this kind of satisfaction of Stem $\equiv \operatorname{PrWd}$ is special to reduplication.

This is in accord with the claim made by Struijke (1998). Investigating reduplication in Kwakwala, where either a certain segment in the input is realized either in the base or in the reduplicant depending on the context in order to avoid a sequence of heavy syllables, she proposes 'Broad IO-faithfulness'. The gist of the idea is that IO-faithfulness constraints are satisfied as long as either the base or the reduplicant is faithful to the input in the relevant dimension. She argues for the necessity of positing a reduplication model along the lines of Spaelti (1997) rather than McCarthy and Prince (1995). Under McCarthy and Prince's full reduplication model, the input has independent correspondence relations with the base and the reduplicant. Given such a model, however, Anchor-L(Stem,PrWd) is clearly violated 
since the relevant stem faithfulness should be evaluated by IO-faithfulness constraints. This shows that the idea of 'Broad IO-faithfulness' is tenable only under a model where the entire reduplication form is compared with the underlying representation.

A related issue is concerned with Contiguity. As can be seen from (11) and (12), complex onsets are not allowed in the reduplicant in Tagalog. This is taken as an effect of the emergence of the unmarked, but avoidance of a *Complex violation results in a violation of Contiguity within the reduplicant domain. Given the fact that Contiguity-Stem is a member of Stem $\equiv \mathrm{PrWd}$ in the sense of propositional logic, this seems to be a problem too. But given the consideration above, Contiguity-Stem is indeed satisfied by (12c) since it is satisfied in the domain of the base. In effect, what Struijke (1998) dubs 'Broad IO-faithfulness' resolves the apparent problem here. Anchor-L(Stem,PrWd) is satisfied by the reduplicant while Contiguity-Stem is fulfilled by the base. In the same vein, Anchor-R(Stem,PrWd) is fulfilled by the base, so all the faithfulness constraints conjoined to obtain the effect of Stem $\equiv \operatorname{PrWd}$ are satisfied by (12c), as encapsulated in (13).

\begin{tabular}{|l|c|c|c||}
\cline { 2 - 4 } \multicolumn{1}{l|}{} & $\begin{array}{c}\text { Anchor-L } \\
\text { (Stem,PrWd) }\end{array}$ & $\begin{array}{c}\text { Anchor-R } \\
\text { (Stem,PrWd) }\end{array}$ & $\begin{array}{c}\text { Contiguity } \\
\text { Stem }\end{array}$ \\
\hline Base & violated & satisfied & satisfied \\
\hline Reduplicant & satisfied & violated & violated \\
\hline Entire form & satisfied & satisfied & satisfied \\
\hline
\end{tabular}

In the suffixal reduplication, the mirror image holds. Contiguity-Stem is still satisfied by the base, but Anchor-L(Stem,PrWd) and Anchor-R(Stem,PrWd) are satisfied by the base and the reduplicant respectively. Finally, infixal reduplication 
can also be accommodated under the idea of 'Broad IO-faithfulness'. Consider the following schematic example: [A-BC-BCD], where the underlined $B C$ is the reduplicant. Anchor-L(Stem,PrWd) and Anchor-R(Stem,PrWd) are both satisfied by the base, and Contiguity-Stem is also satisfied both/either by the reduplicant and/or the base. All modes of reduplication are effectively accommodated.

Second, French (1988:33-34) provides an example where circumfixation cooccurs with reduplication in Tagalog. In [pag-?a-?aral-an] 'will study X' derived from [?aral], pag- -an is an object circumfix, and CV-reduplication takes place additionally. To motivate reduplication, it is necessary that both pag-and -an become invisible for the purpose of evaluating the satisfaction of RM. Essentially the same constraint ranking as in (12) succeeds in obtaining the simultaneous occurrence of circumfixation given that the relevant nonconcatenative change is reduplication.

Essential here is positive (or best-of-the-best) constraint conjunction discussed above. To hide the circumfix from the prosodic word domain, one might suggest that both Anchor-L(Stem,PrWd) and Anchor-R(Stem,PrWd) could be independently assumed as selector constraints, but this is not a viable alternative. As (14) and (15) show, the reason is that either [pag-trabahoh] or [trabahoh-an] is chosen as the sympathy candidate depending on the relative ranking of Anchor-L(Stem,PrWd) and Anchor-R(Stem,PrWd): Anchor-L(Stem,PrWd) »Anchor-R(Stem,PrWd) opts for [trabahoh-an], as in (14), whereas the reverse ranking obtains [pag-trabahoh], as in (15). The problem here is that reduplication is not motivated at all regardless of the sympathy candidate to be chosen because the two possible sympathy candidates satisfy RM by virtue of the fact that they contain either the prefixal portion pag- or suffixal one -an. Thus, the candidate with reduplication incurs gratuitous violations 
of Integrity-IO, and is harmonically bounded by [pag-trabahoh-an]. This argument indicates that the desired sympathy candidate cannot be chosen as long as multiple selector constraints are posited. A single constraint is needed which makes opaque both the prefixal and the suffixal elements at the same time.

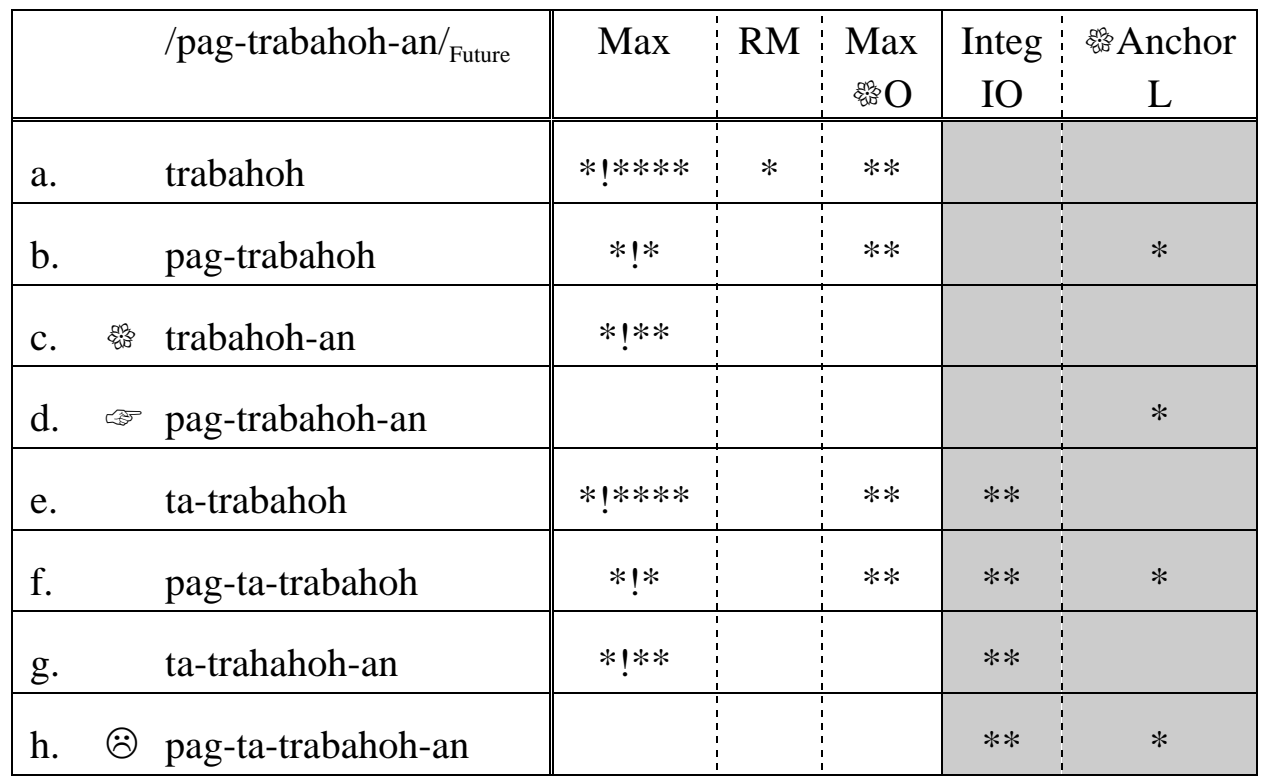

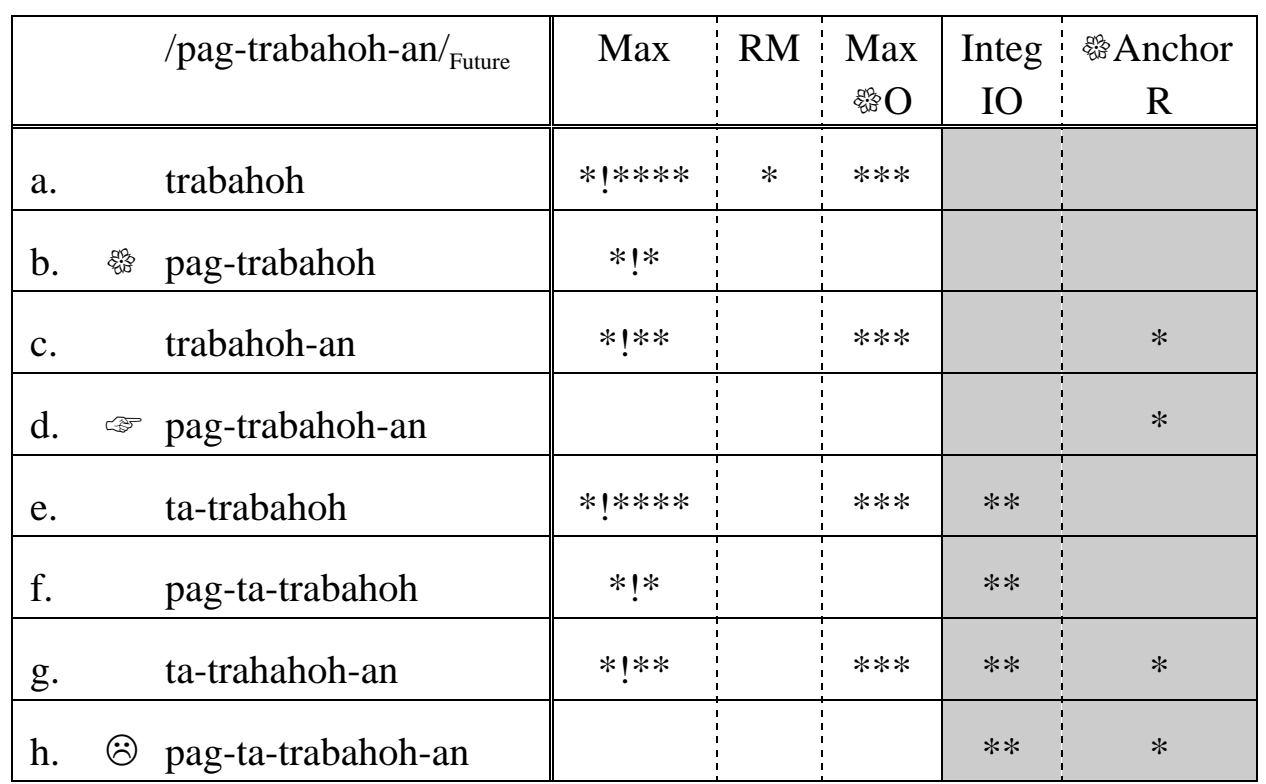


Finally, Clallam, a Salishan language spoken in aboriginal times in many villages along the north coast of Washington's Olympic Peninsula, exhibits the cooccurrence of $i$-infixation and metathesis in the actual aspect formation although it is not quite productive in the language. Examples are given in (16) (Thompson and Thompson 1971:276).

$\begin{array}{lll}\text { Nonactual } & \text { Actual } & \text { Gloss } \\ \text { q'w ut } \int^{\prime \text { suy }} & \text { q'w-(a)2-t } \int \mathrm{u} & \text { lick, beat up } \\ \text { s-(ว)?-yu } & \text { swell up }\end{array}$

Again, these examples are also accommodated by the general schema of DME, as illustrated in (17). Since metathesis is involved, the relevant faithfulness constraint is Linearity.

\begin{tabular}{|c|c|c|c|c|c|c|c|}
\hline & & $/ \mathrm{q}^{\prime \mathrm{w}} \mathrm{ut} \int, \mathrm{P} /_{\text {Actual }}$ & Max & $\mathrm{RM}$ & $\begin{array}{c}\text { Linearity } \\
\\
\end{array}$ & $\begin{array}{c}\text { Linearity } \\
\text { IO } \\
\end{array}$ & $\begin{array}{l}\text { Solem } \\
\equiv \text { PrWd } \\
\end{array}$ \\
\hline $\mathrm{a}$ & & $\mathrm{q}^{\prime \mathrm{w}} \mathrm{ut} \mathrm{J}^{\mathrm{s}}$ & $* !$ & $*$ & * & & \\
\hline $\mathrm{b}$ & & $\mathrm{q}^{\prime \mathrm{w}}-(\mathrm{\partial})$ - $-\mathrm{ut} \int$ & & & $* !$ & & $*$ \\
\hline $\mathrm{c}$ & 8 & $\mathrm{q}^{\prime \mathrm{w}} \mathrm{t} \int \mathrm{u}$ & $* !$ & & & $*$ & \\
\hline d & (x) & $q^{\prime w}-(\partial) 2-t \int u$ & & & & * & * \\
\hline
\end{tabular}

In summary, I argued that DME effects are obtained through sympathy theory in an integrated manner. The key constraint in the theory is the selector constraint. Since the sympathy candidate to be opted for is the one without parsing the underlying affix, I argued that Stem $\equiv$ PrWd serves as the operative selector constraint in morphological opacity. This constraint is formally understood as constraint conjunction of Anchor-L(Stem,PrWd), Anchor-R(Stem,PrWd) and Contiguity-Stem in the sense of propositional logic. This particular interpretation is necessary not only 
to capture DME in all kinds of affixation but also is empirically motivated. The generalized ranking schema of DME presented in (6) generates DME as follows. First, Stem $\equiv$ PrWd requires the sympathy candidate to underparse the affix given in the underlying representation. Due to RM » Faith-IO, the sympathy candidate needs to undergo some stem modification, the specific one depending upon the particular Faith-IO. Given Faithmodificational property of the sympathy candidate, yielding a first phonological exponent. Finally, high ranking Max demands faithful parsing of the underlying affixal element on the surface, obtaining a second phonological exponent.

\subsection{Dominant Affix Effects in Japanese}

Alderete (1999) discusses dominant affix effects in Japanese as an important empirical domain covered by anti-faithfulness theory. Dominant affix effects are phenomena where affixes determine the phonological pattern of the whole word. In this sense, affixes take precedence over roots or stems. Alderete (1999) argues that no standard theoretical machinery of OT can derive them without anti-faithfulness constraints given the universal constraint meta-ranking Faith-Root » Faith-Affix (McCarthy and Prince 1995; Urbanczyk 1996). I claim here that dominant affix effects are subsumed under the rubric of DME, along with various morphological phenomena discussed in the previous section.

\subsubsection{Facts and Descriptive Generalization}

McCawley (1968) and Poser (1984b) observe that Japanese exhibits three types of affix-controlled accentuation processes: dominance effects, preaccentuation, and 
accent shift. Each of these cases is exemplified below in (18). First, the dominant affix - kko requires deletion of the base accent, as in (18a). Thus, the entire word is accentless whether the base contains a lexical accent (as in [kóobe]) or not (as in [edo]). Second, (18b) shows examples of preaccentuation. The suffix -ke demands that the word accent be placed on the syllable immediately preceded by it. As a result, the base-final syllable carries accent both when the base has some original accent (as in [nisímura]) and when the base is accentless (as in [yosida]). Two possible interpretations are available for the /nisímura-ke/ $\rightarrow$ [nisimurá-ke] example: (i) deletion of the original accent plus insertion of a new accent, and (ii) the shift of the original antepenultimate accent to the base-final syllable. I argue that the first interpretation must be rejected in favor of the second one. Finally, (18c) illustrates accent shift. The difference between (18b) and (18c) is that no accent insertion takes place in (18c) while it is obligatory in (18b). In (18c), no accentual change is found when the base carries no original accent, as in /toma-ya/ $\rightarrow$ [toma-ya]. These data are instances of DME since suffixation is accompanied by some suprasegmental change.

(18) a. Dominance effect

$\begin{array}{lll}\text { Underlying } & \text { Output } & \text { Gloss } \\ \text { /kóobe-kko/ } & \text { [koobe-kko] } & \text { native of Kobe } \\ \text { /edo-kko/ } & \text { [edo-kko] } & \text { native of Tokyo }\end{array}$

b. Preaccentuation

\begin{tabular}{|c|c|c|}
\hline $\begin{array}{l}\text { Underlying } \\
\text { /nisímura-ke/ } \\
\text { /yosida-ke/ }\end{array}$ & $\begin{array}{l}\text { Output } \\
\text { [nisimurá-ke] } \\
\text { [yosidá-ke] }\end{array}$ & $\begin{array}{l}\text { Gloss } \\
\text { the Nishimura family } \\
\text { the Yoshida family }\end{array}$ \\
\hline Accent shift & & \\
\hline $\begin{array}{l}\text { Underlying } \\
\text { /kúzu-ya/ } \\
\text { /toma-ya/ }\end{array}$ & $\begin{array}{l}\text { Output } \\
\text { [kuzú-ya] } \\
\text { [toma-ya] }\end{array}$ & $\begin{array}{l}\text { Gloss } \\
\text { junkman } \\
\text { mat seller }\end{array}$ \\
\hline
\end{tabular}




\subsubsection{Analysis}

This section develops an OT analysis of the dominant affix effects exemplified in (18). Beginning with the dominance effect in (18a), in [koobe-kko], accent deletion is a natural outcome if the suffix is made opaque through the selector constraint and accent deletion is employed as the strategy to fulfill RM. The sympathy candidate should be [koobe]. The optimal candidate must imitate the absence of accent in the sympathy candidate because of Dep-o O-Accent. This analysis is demonstrated in (19). There are potentially many other options for a sympathy candidate to satisfy $\mathrm{RM}$, but accent deletion is the most harmonic given $\mathrm{RM} \gg \mathrm{Max}-\mathrm{IO}-$ Accent, assuming that all other IO-faithfulness constraints outrank Max-IO-Accent.

\begin{tabular}{|c|c|c|c|c|c|c|}
\hline & /kóobe-kko/ & Max & $\mathrm{RM}$ & $\begin{array}{c}\text { Dep- } 80 \\
\text { Accent }\end{array}$ & $\begin{array}{r}\text { Max-IO } \\
\text { Accent } \\
\end{array}$ & $\begin{array}{l}\text { Stem } \\
\equiv \text { PrWd } \\
\end{array}$ \\
\hline a. & kóobe & $* ! *$ & $*$ & $*$ & & \\
\hline b. & kóobe-kko & & & $* !$ & & $*$ \\
\hline c. & koobe & $* ! *$ & & & $*$ & \\
\hline d. $\quad$ 吗 & koobe-kko & & & & * & $*$ \\
\hline
\end{tabular}

The same ranking explains [edo-kko], as in (20). This form carries no surface accent, but this is not because of sympathetic correspondence. Since /edo/ lacks an underlying accent, Dep-89-Accent plays no role in the decision of the winner. In (20), RM is assumed to outrank Dep-IO-Accent such that (20c) is selected as the sympathy candidate. Even with this disadvantageous assumption, the correct result is obtained since Dep-se O-Accent is satisfied by (20b). [edó-kko] is eliminated by DepIO-Accent. This constraint does not play any visible role in /kóobe-kko/ since the base already contains an underlying accent. 
(20)

\begin{tabular}{|ll||c|c|c|c|c|c|}
\hline & ledo-kko/ & Max & RM & Dep-\&8O & Dep-IO & Max-IO & \& \\
& & & Accent & Accent & Accent & $\equiv$ PrWd \\
\hline \hline a. & edo & $* ! *$ & $*$ & & & & \\
\hline b. & & & & & & $*$ \\
\hline c. edo-kko & & & & & & \\
\hline d. & edó & $* ! *$ & & & $*$ & & $*$ \\
\hline
\end{tabular}

Second, consider preaccentuation in (18b). The examples show that the suffix -ke demands that the output bear accent on the syllable immediately preceding the suffix regardless of the position and the presence/absence of an underlying accent. Beginning with [yosidá-ke], this example is quite the opposite of [koobe-kko]. The underlying representation of [yosidá-ke] does not carry any accent while the output does. Thus, the operative ranking is obtained if Max-IO-Accent and Dep-so-Accent for the dominance effect are replaced by Dep-IO-Accent and Max-OBO-Accent respectively. As (21) shows, this analysis accounts for [yosidá-ke]. The suffix -ke is made opaque, and the sympathy candidate is required to insert an accent because RM outranks Dep-IO-Accent. Its accentedness becomes crucial given Max-8 O-Accent.

\begin{tabular}{|lc||c|c|c|c|c|}
\hline & /yosida-ke/ & Max & $\mathrm{RM}$ & $\begin{array}{c}\text { Max-\&O } \\
\text { Accent }\end{array}$ & $\begin{array}{c}\text { Dep-IO } \\
\text { Accent }\end{array}$ & $\begin{array}{c}\text { \& } \\
\equiv \text { PrWd }\end{array}$ \\
\hline \hline a. & yosida & $* ! *$ & $*$ & $*$ & & \\
\hline b. & yosida-ke & & & $* !$ & & $*$ \\
\hline c. & $*$ yosidá & $* ! *$ & & & $*$ & \\
\hline d. & mosidá-ke & & & & $*$ & $*$ \\
\hline
\end{tabular}

An important issue here is the locus of accent insertion. Given the ranking in (21), [yósida] and [yosída] are equally qualified as a sympathy candidate, and in 
effect, it is expected that [yósida-ke] and [yosída-ke] are as well-formed as [yosidáke], contrary to fact. My claim is that the base-final syllable carries an inserted accent because the attached affix - $k e$ is a suffix. The suffix -ke and the inserted accent both contribute to the phonological expression of the same morpheme, so they should be realized contiguously on the surface. The locality effect involved in DME is not special to preaccentuation in Japanese. In the plural formation in German, a similar effect is observed: [Palast $]_{\text {Singular }} \rightarrow[\text { Paläste }]_{\text {Plural }}$ (cf. *[Pälaste $], *[$ Päläste $]$ ) (see Wiese 1996b:183-184 for more German examples and discussion). This is formally expressed by a morphemic contiguity constraint such as Morph-Contiguity.

The same constraint ranking does not capture [nisimurá-ke], however. There are three potential forms for the sympathy candidate: [nisímura], [nisimurá], and [nisimura]. Given that [nisimurá-ke] is the optimal form, the sympathy candidate needs to be [nisimurá]. Although [nisímura] loses the selection of the sympathy candidate due to its violation of RM, [nisimura] is a serious competitor. Provided that the final accent in [nisimurá] comes from deletion of the underlying accent plus insertion of a new accent, this form is harmonically bounded by [nisimura], because the latter violates only Max-IO-Accent but still satisfies RM, as shown in (22). This suggests that the deletion-plus-insertion interpretation of [nisimurá(-ke)] is wrong.

\begin{tabular}{|c|c|c|c|c|c|c|}
\hline & /nisímura-ke/ & $\operatorname{Max}$ & $\mathrm{RM}$ & $\begin{array}{c}\text { Max-8 } \mathrm{O} \\
\text { Accent }\end{array}$ & $\begin{array}{c}\text { Max/Dep-IO } \\
\text { Accent }\end{array}$ & $\begin{array}{l}\text { Stem } \\
\equiv \text { PrWd }\end{array}$ \\
\hline a. & nisimurá & $* ! *$ & & & $* *$ & \\
\hline b. & nisimurá-ke & & & & $* * !$ & $*$ \\
\hline c. 8 & nisimura & $* ! *$ & & & * & \\
\hline d. & nisimura-ke & & & & $*$ & $*$ \\
\hline
\end{tabular}


The only possible interpretation of preaccentuation in [nisimurá-ke] is that the underlying accent undergoes movement. From the perspective of Alderete (1999), accent shift incurs a violation of NoFlop. I assume that this is part of the universal constraint set. As demonstrated in (23), the selector constraint chooses [nisimurá] as the sympathy candidate. Under the accent shift interpretation, accentless [nisimura] can be easily ruled out by high ranked Max-IO-Accent. Finally, NoFloprequires the output accent to fall on the syllable immediately before $-k e$. Because NoFlop-IO-Accent does not require a specific position where the original accent is moved to, Morph-Contiguity plays a key role here too.

\begin{tabular}{|lc||c:c|c|c|c|}
\hline & /nisímura-ke/ & Max & $\mathrm{RM}$ & $\begin{array}{c}\text { NoFlop-\&O } \\
\text { Accent }\end{array}$ & $\begin{array}{c}\text { NoFlop-IO } \\
\text { Accent }\end{array}$ & $\begin{array}{c}\text { \& } \\
\equiv \text { PrWd }\end{array}$ \\
\hline \hline a. & nisímura & $* ! *$ & $*$ & $*$ & & \\
\hline b. & nisímura-ke & & & $* !$ & & $*$ \\
\hline c. & nisimurá & $* ! *$ & & & $*$ & \\
\hline d. & m nisimurá-ke & & & & $*$ & $*$ \\
\hline
\end{tabular}

Although the analysis of preaccentuation might appear to depend on two separate rankings, this is not true. In [yosidá-ke], the underlying form does not have any accent, so NoFlop has no room to play a role. By contrast, in [nisimurá-ke], there is an underlying accent, and therefore, Dep-IO-Accent is of no relevance.

Finally, the analysis of accent shift in (18c) is straightforward given the discussion of [nisimurá-ke]. As (24) and (25) show, interactions of NoFlop-IOAccent and NoFlop-so-Accent with other constraints obtain correct output forms. In (25), the underlying representation contains no accent, so all candidates vacuously satisfy the two NoFlop constraints. The relative ranking between RM and Dep-IO- 
Accent selects either (25a) or (25c) as the sympathy candidate, but this issue does not matter here since NoFlop-80 $\mathrm{O}$-Accent is satisfied by all candidates except [tóma] and [tóma-ya], which are ruled out by Dep-IO-Accent.

\begin{tabular}{|c|c|c|c|c|c|c|}
\hline & /kúzu-ya/ & $\operatorname{Max}$ & $\mathrm{RM}$ & $\begin{array}{c}\text { NoFlop-se } \mathrm{O} \\
\text { Accent }\end{array}$ & $\begin{array}{c}\text { NoFlop-IO } \\
\text { Accent } \\
\end{array}$ & 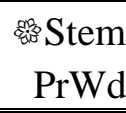 \\
\hline a. & kúzu & $* ! *$ & $*$ & $*$ & & \\
\hline b. & kúzu-ya & & & $* !$ & & $*$ \\
\hline c. & kuzú & $* ! *$ & & & $*$ & \\
\hline d. & kuzú-ya & & & & $*$ & * \\
\hline
\end{tabular}

\begin{tabular}{|c|c|c|c|c|c|c|c|}
\hline & /toma-ya/ & $\operatorname{Max}$ & $\mathrm{RM}$ & $\begin{array}{c}\text { NoFlop } \\
\text { Accent } \\
\end{array}$ & $\begin{array}{c}\text { Dep } \\
\text { IO } \\
\text { Accent } \\
\end{array}$ & $\begin{array}{c}\text { NoFlop } \\
\text { IO } \\
\text { Accent }\end{array}$ & $\begin{array}{l}\text { Stem } \\
\equiv \mathrm{PrWd}\end{array}$ \\
\hline a. & toma & $* ! *$ & $*$ & & & & \\
\hline b. & toma-ya & & & & & & $*$ \\
\hline c. & tomá & $* ! *$ & & & $*$ & & \\
\hline d. & tomá-ya & & & & $* !$ & & * \\
\hline
\end{tabular}

In sum, I discussed that the three types of dominant affix effects involved in Japanese accentuation can be subsumed under the rubric of DME. The constraint rankings offered for the analysis are summarized in (26). It is clear that all the data in (18) are explained in a unified way by the general ranking schema in (6). The only variables are Faith-IO and Faith- $\mathrm{O}$. They are replaced by different faithfulness constraints depending on the kind of affix. Finally, it should be made explicit that each faithfulness constraint must inherently bear the type marking of the relevant affix, because the ranking of Max-IO-Accent, for example, varies from affix to affix. 


\begin{tabular}{|c|c|c|}
\hline & Affix-specific ranking & Common ranking \\
\hline Dominance effect & $\begin{array}{l}\text { Dep- } \$ O \text { O Max-IO } \\
\text { RM » Max-IO }\end{array}$ & \multirow{3}{*}{$\begin{array}{c}\text { Max } \gg \text { Stem } \equiv \text { PrWd } \\
\text { Faith-s } \gg \text { Faith-IO } \\
\text { RM } \gg \text { Faith-IO }\end{array}$} \\
\hline Preaccentuation & 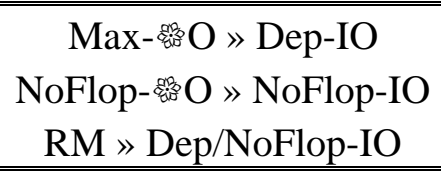 & \\
\hline Accent shift & $\begin{array}{c}\text { NoFlop-- } \mathrm{O} \text { » NoFlop-IO } \\
\text { RM » NoFlop-IO }\end{array}$ & \\
\hline
\end{tabular}

\subsection{The Impersonal Formation in Chaha}

The impersonal formation in Chaha, a Western Gurage language of the Southern Ethiopic group, exhibits an interesting type of DME. Unlike the examples discussed so far, there is no apparent affix in the surface representation, and the impersonal morpheme is expressed in four varieties: (i) both labialization and palatalization, (ii) only labialization, (iii) only palatalization, or (iv) no surface phonological realization. DME is thus observed when the morpheme is realized both by labialization and by palatalization simultaneously. The principal issue is why two realizations appear despite the fact that no surface transparent affix exists. I argue that the underlying representation indeed does contain an affix. I also demonstrate that cases where only labialization or palatalization occurs are explained by high ranked markedness constraints which suppress the function of RM or Max.

\subsubsection{Facts and Descriptive Generalization}

The phonology and morphology surrounding the impersonal formation in Chaha have attracted a substantial body of earlier attention both descriptively and theoretically (Polotsky 1938; Leslau 1950; 1967; Hetzron 1971, 1977; Johnson 1975; McCarthy 1983, 1986a; Lieber 1988; Elmedlaoui 1992; Rose 1994, 1997). The impersonal is 
primarily used when the subject is inexplicit like English one, although this is by no means the exhaustive context (see Leslau 1967:1150-1151 for documentation of other contexts). This section provides the entire set of facts and descriptive generalization of the impersonal secondary articulations.

The impersonal is characterized most prominently by the simultaneous occurrence of labialization and palatalization, although their cooccurrence does not always hold. Labialization targets only labial and dorsal consonants while potential legitimate targets of palatalization are coronal and dorsal obstruents. This generalization is quite robust and holds of other morphological formations in the language, including the second person singular feminine imperative, the third person singular masculine perfective with objects, and the second person singular feminine subject. Another strong general tendency across these paradigms is that palatalization targets only the rightmost consonant of a root or stem whereas labialization exhibits a more flexible and unbounded character in that the rightmost eligible consonant serves as the target. These two generalizations cover most of the data of concern. Examples are given in (27). Palatalization of coronal obstruents turns them into palato-alveolars (Johnson 1975; Rose 1994, 1997), but I keep using a superscripted [ $\left.{ }^{\mathrm{y}}\right]$ for perspicuity.

Personal Impersonal Gloss

a. kafat nakas dəmad tabas tozrabət

$\operatorname{kaf}^{\mathrm{w}} \partial \mathrm{t}^{\mathrm{y}}$ $n \partial k^{\mathrm{w}} \partial \mathrm{s}^{\mathrm{y}}$ dəm ${ }^{\mathrm{w}} \partial \mathrm{d}^{\mathrm{y}}$ $\operatorname{tab}^{\mathrm{w}} \partial \mathrm{s}^{\mathrm{y}}$ $\operatorname{tazrab}^{\mathrm{w}} \mathrm{at}^{\mathrm{y}}$

b. nəkəb

$\mathrm{s}^{\mathrm{y}}$ anวb məkər bənar qatar nəkəb ${ }^{\mathrm{w}}$ $\mathrm{s}^{\mathrm{y}}$ anab ${ }^{\mathrm{w}}$ mak ${ }^{\mathrm{w}}$ or $b^{\mathrm{w}}$ ənวr $q^{\text {watər }}$ open

bite

join

fry have hope for someone

find

spin advise demolish kill 


\begin{tabular}{|c|c|c|}
\hline sant & sant $^{\mathrm{y}}$ & force through \\
\hline $\begin{array}{l}t^{\mathrm{y}} \mathrm{af}^{\mathrm{w}} \partial \mathrm{rr} \\
\mathrm{g}^{\mathrm{y}} \partial \mathrm{k}^{\mathrm{y}} \partial \mathrm{r}\end{array}$ & $\begin{array}{l}t^{\mathrm{y}} \mathrm{af}^{\mathrm{w}} \partial \mathrm{r} \\
\mathrm{g}^{\mathrm{y}} \partial \mathrm{k}^{\mathrm{y}} \partial \mathrm{r}\end{array}$ & $\begin{array}{l}\text { scratch and mark } \\
\text { straighten out }\end{array}$ \\
\hline $\begin{array}{l}\text { bətəx } \\
\text { axwonəq } \\
\text { dənəg }\end{array}$ & 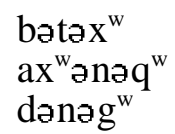 & $\begin{array}{l}\text { dig out } \\
\text { take off the clothes } \\
\text { hit }\end{array}$ \\
\hline
\end{tabular}

First, both labialization and palatalization take place when the stem-final segment is a coronal obstruent. Palatalization occurs stem-finally whereas labialization is realized on the rightmost available consonant, as in (27a). Second, palatalization fails to appear whenever the final consonant is not a proper segment. Rather, only labialization occurs, as shown in (27b). Unlike palatalization, however, labialization is permitted flexible mobility and targets the rightmost available segment, although labialization shares the right-edge orientation with palatalization. Third, only palatalization is observed when all consonants are coronals, as exemplified in (27c). Fourth, neither labialization nor palatalization takes place if the rightmost consonant is labial or sonorant and no segment is a proper docking site of labialization because of underlying secondary articulations, as shown in (27d). I simply assume that an articulatorily motivated feature cooccurrence constraint against $*[[\mathrm{Lab}][\mathrm{Cor}]]_{\mathrm{Seg}}$ is operative, and I do not discuss the examples in (27d) any further. When the stem-final segment is a dorsal consonant, labialization takes precedence to palatalization, as exemplified in (27e). Since palatalization strictly targets a stemfinal consonant, this labial priority entails the failure of palatalization in such cases.

\subsubsection{Morphemic Representation of the Impersonal}

It is clear from the data presented in (27) that no surface affix exists in the impersonal formation in Chaha. This appears to be problematic for the general proposal for 
DME because the selector constraint makes only affixes opaque such that the pressure of RM forces some phonological change on the stem. In this section, I argue that there exists an affix in the underlying representation. For this purpose, I critically review various claims made in the earlier literature.

Polotsky (1938) and Leslau (1967) attempt to provide a historical account for the emergence of labialization and palatalization in the impersonal. The gist of their argument is that labialization came from the Proto-Ethiopic plural marker $-u$ whereas palatalization originated from the object suffix pronoun $-i$. The impersonal accompanies various different suffixes expressing the object type, but the impersonal obligatorily carries the third person singular masculine heavy object marker $-i$ when no such suffix exists. Polotsky and Leslau argue that the $i$-induced palatalization process was morphologized and pervaded the impersonal paradigm regardless the type of the object, resulting in the contemporary palatalization phenomenon in the whole paradigm. However, it is entirely unclear why the plural marker and the object suffix pronoun together contribute to the impersonal formation. The semantic content of the impersonal is never obtained from their compositionality. The impersonal morpheme is an indivisible atomic primitive.

Hetzron (1971) gives a different diachronic account. His position is that the third person plural masculine suffix $-u u$ was developed into the impersonal. He posits some steps to derive both labialization and palatalization from this single source. First, the suffix underwent shortening, and compensatory labialization occurred on the stem. Second, dissimilation took place between the secondary labial articulation and the suffix $-u$, and the latter changed into $-i$. Finally, the suffix $-i$ came to be realized as palatalization of a stem consonant. This proposal is attractive for two 
reasons. First, labialization and palatalization come from one and the same source, so the problem encountered by Polotsky (1938) and Leslau (1967) is overcome. Second, Hetzron's analysis provides a natural account for the reason why labialization is granted priority when a stem-final consonant is dorsal, thereby labialization and palatalization compete with each other for the segmental position as in (27e). Since labialization came first historically, the dorsal position was already occupied by labialization when palatalization came into the impersonal. In spite of these strengths, Hetzron's historical study does not offer a significant insight into the synchronic system of the impersonal formation. Adopting his analysis would be tantamount to saying that the impersonal formation is not governed by any systematic morphological and phonological regularity from a synchronic point of view. The secondary articulation process is quite productive and systematic, and thus, the robust generalization would be a synchronic accident.

McCarthy (1983), Lieber (1988), and Rose (1994) tackle the impersonal formation from a generative point of view. Despite the fact that their specific details of analyses are different, they share the idea that the secondary articulations are attributed to floating autosegmental features in the underlying representation. Docking of these two autosegmental features on stem consonants is regulated by language-specific rules, and they are phonetically uninterpretable when there is no proper docking site. Their analyses give a synchronic analysis of the impersonal formation, and the use of autosegmental elements in the underlying representation turns out to be a significantly useful analytical device since the emergence of autosegmental phonology (Goldsmith 1976). But I argue that their analyses are not on the right track. 
First, the equal status accorded to the two floating features in the underlying representation fails to capture a number of crosslinguistic asymmetries between labialization and palatalization attested in a wide range of the tt-group Gurage languages, which include the following four families: Gurage (Muher), Western Gurage (Masqan), Central Western Gurage (Ezha, Chaha, Gumer, and Gura), and Peripheral Western Gurage (Gyeta, Inor, Endegen, and Ener). First, the impersonal formation in Muher is associated only with stem-final palatalization, but there is no language in which only labialization expresses the impersonal morpheme (Hetzron 1971:195). In all other languages enumerated above, both labialization and palatalization participate in the impersonal formation.

Second, Hetzron (1971:196) notes that final palatalization always entails internal labialization in Inor and Ener. This indicates that palatalization is primary while labialization is subsidiary, but not vice versa. Moreover, in all Peripheral Western Gurage languages except Gyeta, palatalization affects the third person plural feminine morpheme without labialization.

Finally, as listed in (28), internal labialization takes place with a suffix in various word formations in extensive Gurage languages, but palatalization does not (Hetzron 1971:194-196). In (28), 'IL' indicates internal (i.e., word-medial) labialization. These contrasts between palatalization and labialization strongly pose a suspicion that they should not be treated on the same ground.

(28) a. Third person singular masculine light object:

$\mathrm{IL}+\mathrm{n}$ (Masqan, Central Western Gurage, Gyeta)

b. $\quad$ Third person singular masculine light L:

$\begin{array}{llll}\text { IL+rä/lä } & \text { (Chaha, Ezha) } & \text { IL+rä } & \text { (Gura) } \\ \text { IL+lä } & \text { (Gumer) } & \text { IL+nä } & \text { (Gyeta) }\end{array}$


Second, and more convincingly, another asymmetry resides in the behaviors in terms of phonological locality. Presumably related to the first set of arguments, palatalization is most generally (though not strictly) restricted to the right edge of a stem across the Gurage languages, but labialization displays a more unbounded character. This is true of the impersonal in Chaha as seen from (27), but some independent evidence is given in (29). As with the impersonal, only coronal and dorsal obstruents qualify as docking sites of secondary palatalization while labials and dorsals serve as proper docking sites of secondary labialization. The second person singular feminine imperative is expressed solely by palatalization, but it fails to appear if the final segment is illegitimate for palatalization, as in (29a), even if an appropriate segment exists word-initially or word-medially. By contrast, the third person singular masculine perfective with objects is denoted only by labialization. Unlike palatalization, labialization occurs anywhere as long as the segment is the rightmost eligible one, as in (29b). It remains phonetically unrealized only when no segment is a legitimate target as in [sædæd]. This locality contrast is inexplicable if labialization and palatalization have the same status. Rose's (1997) analysis assuming two discontinuous suffixes /-u, -i/ likewise suffers from the same problems.

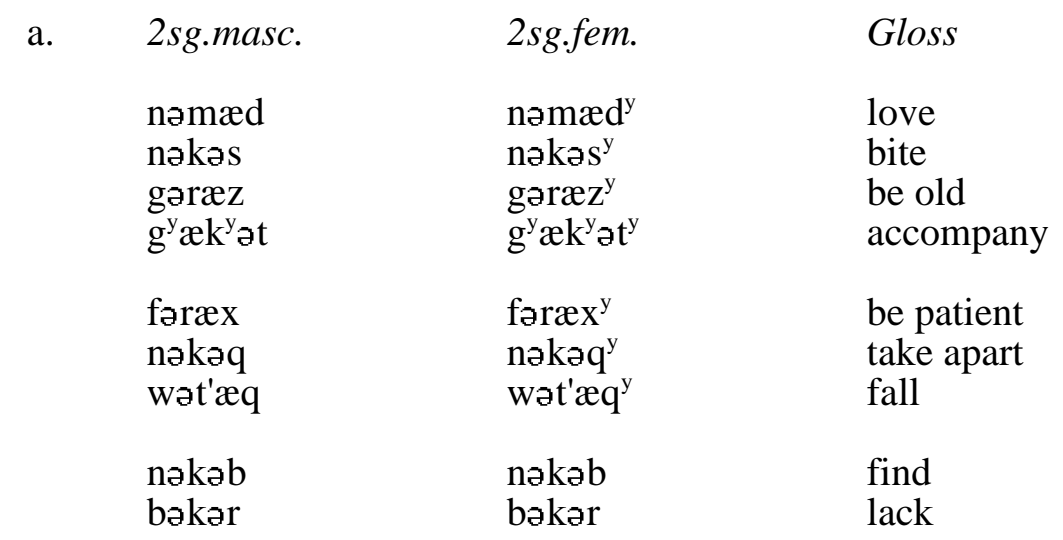


b. Perfective 3sg.masc.

\begin{tabular}{|c|c|c|}
\hline Without object & With Object & Gloss \\
\hline dænæg & dænæg $^{\mathrm{w}}$ & hit \\
\hline nædæf & nædæf $\mathrm{f}^{\mathrm{w}}$ & sting \\
\hline nækæb & nækæb $^{\mathrm{w}}$ & find \\
\hline nækæs & næk $^{\mathrm{w}} æ s$ & bite \\
\hline bækær & $\mathrm{b}^{\mathrm{w}} æ \mathrm{r}$ & lack \\
\hline kæfæt & $\mathrm{kæf}^{\mathrm{w}} \mathfrak{x t}$ & open \\
\hline qæfæt & $\mathrm{q}^{\mathrm{w}} æ f \mathfrak{t}$ & kill \\
\hline mæsær & $\mathrm{m}^{\mathrm{w}} æ s æ r$ & seem \\
\hline $\mathrm{mæk}^{\mathrm{y}} \mathrm{x}$ & $\mathrm{m}^{\mathrm{w}} \mathfrak{x k}^{\mathrm{y}} \mathfrak{x}$ & burn \\
\hline sædæd & sædæd & chase \\
\hline
\end{tabular}

My proposal is that the impersonal morpheme has only /i/, and an appropriate alignment constraint determines the affixal position as a suffix. On the other hand, labialization does not come from any direct phonological source in the underlying representation but from interactions of constraints. This proposal directly captures the various asymmetries between labialization and palatalization discussed above. First, it makes direct sense that only palatalization participates in the impersonal in Muher and that all other $t t$-group Gurage languages employ both labialization and palatalization in the phonological manifestation of the impersonal morpheme. Under the proposal here, palatalization occupies a more central position than labialization, so the lack of labialization without palatalization is directly captured whereas palatalization can occur independently of labialization. Second, the entailment relationship found in Inor and Ener (i.e., labialization always entails palatalization) receives a natural understanding in the same manner if palatalization comes from a real affix but labialization does not. Third, the non-existence of the cooccurrence of a suffix and palatalization also follows from my proposal. The source of palatalization is a phonologically full-fledged segment in the underlying representation, and 
therefore, no other affix has room to occupy the same suffixal position. But labialization does not derive from any such underlying affixal segment, meaning that some independent affixal segment does not need to compete for a single suffixal slot. Finally, the locality contrast is easily explained by the underlying contrastive status of palatalization and labialization too. The strict right edge orientation of palatalization is considered as the effect to preserve the suffixal feature associated with the relevant alignment constraint, but the locus of labialization is not inherently fixed since it does not have any phonological source from which it is derived. In conclusion, these various differences holding of labialization and palatalization naturally follow if palatalization is granted the status of an authentic affix (i.e., /i/) and if labialization is not. This proposal plays a central role in the subsequent discussion.

\subsubsection{Selector Constraint Revisited}

I argued that the morphemic representation of the impersonal merely consists of an affix /i/. The immediate question is how labialization is additionally obtained. In this section, I demonstrate that the selector constraint (i.e., Stem $\equiv \operatorname{PrWd}$ ) cannot accommodate the Chaha data as it stands, and propose a slightly extended but more stringent version of the selector constraint.

Labialization is considered as an instance of additional morphemic exponence. Under the proposal advanced here, this is required because the existence of the affix /i/ is made opaque. However, the affix is realized as a secondary articulation, and therefore, no overt affix exists in the output form. This gives rise to an important question: how is labialization motivated? In all cases considered so far, there exists

some element made opaque through the selector constraint. The nature of Anchor 
becomes crucial at this point. According to the definition of the constraint given by McCarthy and Prince (1995:371), all that is needed for the satisfaction of this constraint is that any element at the designated edge (left or right) of one category has a correspondent at the same edge of another category. Thus, Anchor(Stem,PrWd) is satisfied even if palatalization affects the rightmost segment of a stem. Since the affixal element /i/, which contributes to the satisfaction of RM, is incorporated in the stem domain, as schematized in (30), the stem domain is coextensive with the prosodic word domain. It is important that the selector constraint can make part of an output element invisible but not an underlying element since the impersonal morpheme contains a segmental affix in the underlying representation. This holds since OT constraints are output-oriented.

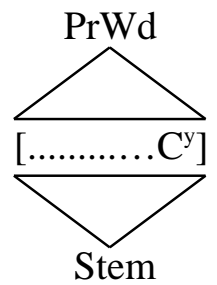

The structure in (30) created through palatalization satisfies both RM and Stem $\equiv$ PrWd by merging the stem-final segment and the affixal segment of the impersonal morpheme, which are affiliated with distinct morphemes. This means a violation of Morphological Uniformity that militates against underlying elements belonging to different morphemes from collapsing phonologically such that they are realized within one phonological element (Sanders 1999). Particularly, the pertinent phonological element here is a segment so that underlying segments affiliated with different morphemes must be instantiated heterosegmentally. It is essential here to define Morphological Uniformity with reference to underlying elements. 
Labialization occurs in the impersonal formation in Chaha, and it is obviously parasitic upon a stem segment. Given that labialization is completely morphological, it is associated with the impersonal morpheme rather than with a stem morpheme. Given the proposal in the preceding section, however, labialization does not possess any phonological substance as an underlying source, and therefore, the presence or absence of the relevant phonological content in the underlying representation is what differentiates palatalization from labialization. Labialization would incur a violation of Morphological Uniformity unless the input-output mapping is taken into account. Since Uniformity is a faithfulness constraint, the sensitivity of Morphological Uniformity to the input naturally follows. Morphological Uniformity can be now defined as in (31).

(31) Morphological Uniformity

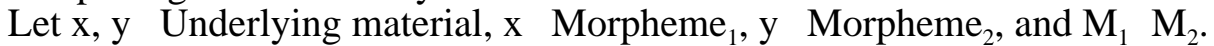
Then, Morphological Uniformity is satisfied iff $\mathrm{x}$ and $\mathrm{y}$ are segmentally independent.

Morphological Uniformity as defined here is similar to Morphemic Disjointness proposed by McCarthy and Prince (1995:310), but they are not quite the same. The definition of Morphemic Disjointness is given in (32), where morpheme associate is considered as follows: a segment (autosegment) $\mathrm{x}$ is an associate of morpheme $\mathrm{M}_{\mathrm{k}}$ if $\mathrm{x}$ or some correspondent of $\mathrm{x}$ is an exponent of $\mathrm{M}_{\mathrm{k}}$, expressed as $\mathrm{x} \oint \mathrm{M}_{\mathrm{k}}$ ). Morphemic Disjointness maintains that distinct instances of morphemes have distinct phonological contents like Morphological Uniformity.

(32) Morphemic Disjointness

$\mathrm{x}\left\{\mathrm{M}_{\mathrm{i}} \rightarrow \mathrm{x} \mid \mathrm{M}_{\mathrm{j}}\right.$, for instances of morphemes $\mathrm{M}_{\mathrm{i}} \neq \mathrm{M}_{\mathrm{j}}$ and for $\mathrm{x}$ a specific segmental (autosegmental) token.

"Distinct instances of morphemes have distinct contents, tokenwise." 
McCarthy and Prince espouse Morphemic Disjointness in the context of phonological merger concomitant with reduplication in languages such as Chumash. In the Chumash word [sik-sikuk] meaning 'he is chopping/hacking', the initial sik is the reduplicant of the base, but the initial segment $s$ corresponds to the phonological prefix of the third person singular morpheme as well. In this context, reduplication denotes the continuative. The crucial difference between Morphological Uniformity and Morphemic Disjointness is that the former is sensitive only to the phonological material already present in the underlying representation while the latter is not. Because the reduplicant does not carry any concrete underlying phonological substance, Morphological Uniformity as defined in (30) is satisfied by [sik-sikuk] in Chumash. In this sense, the coverage of Morphological Uniformity is a subset of that of Morphemic Disjointness. In Chaha impersonals, labialization parasitic on a stem segment violates Morphemic Disjointness, but it satisfies Morphological Uniformity.

I propose that Morphological Uniformity must be incorporated as part of the definition of the selector constraint (i.e., Stem $\equiv$ PrWd). This is achieved by conjoining it with Stem $\equiv$ PrWd already established in section 5.2 (see (9)). The net effect of the integration of Morphological Uniformity within the definition of the selector constraint is that squeezing an affix in the form of secondary articulation or coalescence is prohibited to the end of keeping an affix visible. Such a makeshift resolution of the offending problem is not allowed. This extension seems necessary independently of the impersonal formation in Chaha since such brute-forced satisfaction of RM and Stem $\equiv$ PrWd must be prohibited in all cases discussed thus far.

Besides the proposed solution, another possibility is conceivable, paying attention to the fact that palatalization affects the phonological make-up of a stem: 
conjunction of Stem $\equiv$ PrWd and Ident-IO-[F]. The idea is that setting aside an affix outside the prosodic word domain is not sufficient but stem segments must not undergo any featural changes. Assuming best-of-the-best conjunction here, the case at hand might be tenable. However, this alternative comes across serious problems. First, this identity-based approach cannot distinguish labialization and palatalization since the presence or absence of their underlying source does not matter for the computation of Ident-IO-[F]. This is an empirical problem specific to the impersonal formation.

A more general and serious problem arises once Ident-IO-[F] is conjoined with Stem $\equiv$ PrWd, however. Consider a hypothetical DME example in which the underlying $/ \mathrm{ABC}-\mathrm{D}_{\alpha} /_{\alpha}$ surfaces as $[\mathrm{ABC} \mathrm{D}]_{\alpha}$. The three segments $A B C$ are members of a stem and $D$ is a suffixal segment, and $/ \mathrm{C} / \rightarrow\left[\mathrm{C}^{\prime}\right]$ involves some Ident-IO-[F] violation. If the best-of-the-best conjunction of Stem $\equiv \mathrm{PrWd}$ and Ident-IO-[F] is posited as the selector constraint, the desired output never wins. As demonstrated in (33), this is because (33c) crucially violates the selector constraint and (33a) is chosen as the sympathy candidate. (33d) is harmonically bounded by (33b). This shows that DME never emerges when the stem modification incurs an Ident-IO-[F] violation, contrary to fact (e.g., umlaut in German plurals).

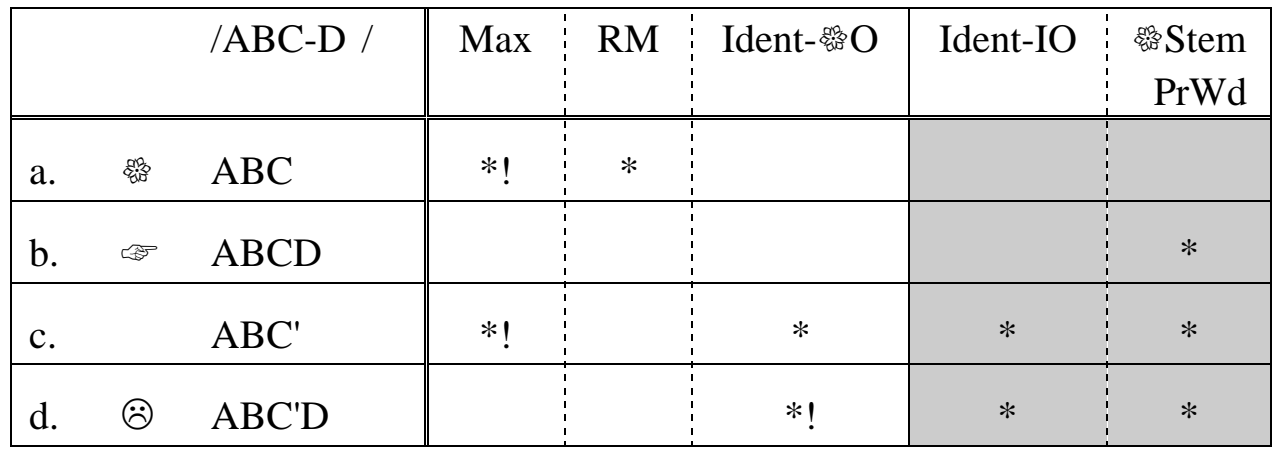




\subsubsection{Analysis}

In sections 5.4.2 and 5.4.3, I set up the background on which my analysis is constructed. This section demonstrates how the set of data in (27) is accounted for. Before going into it, however, I establish several undominated constraints. Since labialization and palatalization never cooccur with coronal and labial segments respectively, I assume $* \operatorname{Cor}^{\mathrm{w}}$ and ${ }^{*} \mathrm{Lab}^{\mathrm{y}}$ as undominated constraints. Furthermore, palatalization occurs on only a subset of coronal consonants: obstruents. This can be captured through an antagonistic phonological markedness constraint $*[+ \text { Son, Cor }]^{\mathrm{y}}$. Finally, palatalization targets only a final segment of a stem (or a root-final segment). This generalization is captured by inviolable Align- $\mathrm{R}(\mathrm{Pal}, \mathrm{PrWd})$. These constraints are never violated in the impersonal, so we can safely assume that they are all undominated. Hence, I do not consider candidates violating any of them.

Another background concerns the driving force of palatalization. As I argued in section 5.4.2, palatalization originates from the underlying impersonal affix /i/, but what causes its surface realization as a secondary articulation? Rose (1997) addresses the same question, and argues that Anchor-Root is pertinent. This constraint requires the affixal element to be segmentally anchored on the stem to which it is attached in the underlying representation. Some other promising analytical possibilities come to our mind, but pursuing the best constraint is beyond the scope of the interest here, so I simply assume that the Anchor constraint is operative. The underlying full segment of the impersonal affix surfaces parasitically as a secondary articulation when it is parsed in the output, so the Anchor constraint is also considered to be undominated.

I begin my analysis with the central examples in (27a) in which both labialization and palatalization occur. The descriptive idea is that labialization is 
called for because Anchor-induced palatalization leads to a violation of Stem $\equiv \mathrm{PrWd}$. The reason why labialization is employed as a secondary exponent is considered to be the result of RM » Dep-IO-[Round]. As illustrated in (34), this analysis picks up (34b) as the sympathy candidate. This form exerts its influence over other candidates through Max-8 $\mathrm{O}-[$ Round] such that (34c) is crucially eliminated here. (34c) and (34d) satisfy Max-IO-Seg because they preserve the affixal segment in the form of palatalization, whereas the underlying segment is completely obliterated in (34a) and (34b). Given this analysis, the double secondary articulations are now understood as follows. First, palatalization has its source in the impersonal affix, and the affixal full segment is forced to appear as a secondary articulation due to the inviolable pressure of Anchor-Root. Second, palatalization necessarily leads to a violation of the selector constraint in the sense discussed in the previous section, so the existence of palatalization is disregarded for the computation of the satisfaction/violation of RM. Among various potential strategies to satisfy RM, labialization costs the least because of RM » Dep-IO-[Round]. The sympathy candidate bears this property. Finally, high ranked Maxsecondary articulations.

\begin{tabular}{|c|c|c|c|c|c|c|c|}
\hline & & /kəfət-i/Impersonal & Max & $\mathrm{RM}$ & $\begin{array}{c}\mathrm{Max}-{ }^{6} \mathrm{O} \\
{[\mathrm{Rd}]} \\
\end{array}$ & $\begin{array}{c}\text { Dep-IO } \\
{[\mathrm{Rd}]} \\
\end{array}$ & $\begin{array}{l}\text { S Stem } \\
\equiv \text { PrWd } \\
\end{array}$ \\
\hline a. & & kafat & $* !$ & $*$ & $*$ & & \\
\hline & 89 & $\mathrm{kaf}^{\mathrm{w}} \partial \mathrm{t}$ & $* !$ & & & $*$ & \\
\hline c. & & $\operatorname{kafat}^{\mathrm{y}}$ & & & $* !$ & & $*$ \\
\hline d. & 喅 & $\operatorname{kof}^{\mathrm{w}} \partial \mathrm{t}^{\mathrm{y}}$ & & & & $*$ & $*$ \\
\hline
\end{tabular}


Consider the examples in (27b). In these cases, the stem-final segments are either a labial or a coronal sonorant, so palatalization cannot target them. Given the undominated Align-R(Pal,PrWd) constraint, the underlying affixal segment fails to surface at the expense of a Max violation. Leaving out the candidates violating any of the undominated constraints discussed above leaves only two candidates. Given $\mathrm{RM} \gg$ Dep-IO-[Round], (35b) is more harmonic. Note that [nək ${ }^{\mathrm{w}} \mathrm{\partial b}$ ] also passes all undominated constraints but is eliminated by Anchor-R(Lab,PrWd). Given the last three forms in (27b), it is obvious that RM outranks this Anchor constraint. Since both candidates in (35) satisfy the selector constraint, sympathetic correspondence plays no tangible role.

\begin{tabular}{|ll||c:c:c|c|c|}
\hline & /nəkəb-i/Impersonal & Max & $\mathrm{RM}$ & $\begin{array}{c}\text { Max-\&O } \\
{[\mathrm{Rd}]}\end{array}$ & $\begin{array}{c}\text { Dep-IO } \\
{[\mathrm{Rd}]}\end{array}$ & $\begin{array}{c}\text { \& Stem } \\
\equiv \mathrm{PrWd}\end{array}$ \\
\hline \hline a. & nəkəb & $*$ & $* !$ & $*$ & & \\
\hline b. & $*$ & & & $*$ & \\
\hline
\end{tabular}

The examples in (27c) are trivial, and do not deserve any special comment. Finally, consider (27e). In these examples, the interesting observation is that a final dorsal segment is occupied by labialization despite the fact that dorsal consonants are available docking sites for palatalization as well. The distribution of palatalization is strictly restricted to the rightmost consonant of a stem, so labialization in this context entails the failure of parsing the underlying affixal segment. Following Rose (1997:112), my analysis employs a markedness consideration. Palatalized coronals are universally preferred to palatalized dorsals (cf. Maddieson 1984). This universal tendency is expressed as $* \operatorname{Dor}^{\mathrm{y}} \gg$ Max $» * \operatorname{Cor}^{\mathrm{y}}$. The contrast that a final dorsal is blocked from palatalization but a final coronal is not is captured by this ranking. 
As demonstrated in (36), this ranking succeeds in generating the correct result. McCarthy (1983), Lieber (1988), and Elmedlaoui (1992) assume in their derivational analyses that labialization takes precedence to palatalization. On the other hand, Rose (1994) provides a historical account, maintaining that only coronal obstruents were palatalizable at an early stage of the language. Rose (1997) argues against these analyses. By contrast, the analysis here gives a synchronic explanation which is intrinsically motivated across languages. As discussed in section 5.4.2, a synchronic account is superior to a historical conjecture to understand the current system of the impersonal formation in Chaha.

\begin{tabular}{|c|c|c|c|c|c|c|c|}
\hline & /batax-i/Imbersonal & $*$ Dor $^{y}$ & Max & $\mathrm{RM}$ & $\begin{array}{c}\mathrm{Max} \\
\mathrm{B}_{0} \mathrm{O} \\
{[\mathrm{Rd}]}\end{array}$ & $\begin{array}{c}\text { Dep } \\
\text { IO } \\
{[\mathrm{Rd}]} \\
\end{array}$ & $\begin{array}{l}\text { Stem } \\
\equiv \mathrm{PrWd}\end{array}$ \\
\hline a. & batax & & $*$ & $* !$ & $*$ & & \\
\hline b. & bata $x^{y}$ & $* !$ & & & $*$ & & * \\
\hline c. & bətəx ${ }^{w}$ & & $*$ & & & $*$ & \\
\hline d. & $\mathrm{b}^{\mathrm{w}} \operatorname{atax} \mathrm{x}^{\mathrm{y}}$ & $* !$ & & & & $*$ & $*$ \\
\hline
\end{tabular}

Summing up, I argued that the double secondary articulations observed in the impersonal formation in Chaha is an instance of DME. The impersonal morpheme possesses a full segment /i/ underlyingly, and it is realized as palatalization by Anchor-Root. Labialization is required additionally, but it does not come from any underlying phonological source unlike palatalization. Rather, labialization is motivated and obtained through interactions of constraints. Essential in the analysis above is the extension of the selector constraint. As discussed in section 5.4.3, the idea of Morphological Uniformity needs to be incorporated as part of the definition of Stem $\equiv$ PrWd. Finally, blocking of DME is due to high ranked markedness 
constraints. Palatalization is prevented when a relevant markedness constraint ranked over Max is active whereas labialization is blocked if the stem consonants consist only of coronal segments.

\subsection{Affixation-Reduplication Interactions in Tagalog}

Following the discussion thus far developed, this section discusses DME in Tagalog. Affixation and reduplication are both fully integrated in the morphology of the language and are used quite productively to express various different morphosyntactic functions. Tagalog morphology presents strong evidence that DME is authentic in natural languages in that their cooccurrence is indispensable to make explicit the specific morphosyntactic category intended by the speaker. I analyze relevant data to support the argument developed so far. In addition, the discussion here lends endorsement for morphosyntactic markings on faithfulness constraints.

\subsubsection{Multiple Functions of Affixation and Reduplication}

Tagalog has a rich inventory of affixes, and takes advantage of reduplication quite productively. An interesting fact is that affixation and reduplication are often combined to express a particular morphosyntactic category (Carrier 1979, 1984; Lieber 1981; McCarthy 1981; Marantz 1982; French 1988). A paradigm involving a verbal stem (trabahoh 'work' and isda: 'fish') and an agentive prefix (mag-/nagImat-/nat-) is provided in (37) (French 1988:23). These examples show two prominent facts of Tagalog morphology. First, a phonologically identical prefix is used for more than one morphosyntactic function: mag-and mat- are used both in the basic aspect and in the future aspect, whereas nag- and nat- are used in the 
completive and the continuative aspects. This suggests that the prefix cannot indicate a unique morphosyntactic category. Second, the same reduplicant shape is recycled: CV-reduplication is employed both by the future aspect and by the continuative aspect. This indicates that the presence/absence of reduplication alone is not sufficient either to disambiguate various morphosyntactic categories.

Affixed/reduplicated forms Gloss

a. mag-trabahoh mag-ta-trabahoh nag-trabahoh nag-ta-trabahoh

work (basic aspect) will work (future aspect) worked (completive aspect) be working (continuative aspect)

b. may-?isda? may-?i-?isda? nay-?isda? nay-2i-2isda?

fish (future aspect) will fish (future aspect) fished (completive aspect) be fishing (continuative aspect)

More examples are given in (38) to reinforce the same point. Note in particular that the agent prefix mag- is used for multiple morphosyntactic functions: the future aspect, moderative verbs (and intensive verbs). Again, this strongly indicates that this prefix is not sufficient to refer to a particular morphosyntactic category. We also find three types of reduplicant shapes: CV (gerunds and occupational nouns), CVV (the future aspect and causative adjectives), and two syllables (moderative verbs and intensive verbs). Carrier $(1979,1984)$ argues that permitted reduplicant shapes are restricted to these three in Tagalog, although disyllabic reduplication has a further ramification, as will be discussed in section 5.5.2. Since reduplication occupies a central place in Tagalog morphology and is employed quite regularly and productively across a variety of morphosyntactic functions, the same reduplicant shape is unavoidably recycled frequently. 
a. Gerunds

$\begin{array}{lll}\text { Base form } & \text { Reduplication form } & \text { Gloss } \\ \text { (um)-laakad } & \text { pag-la-laakad } & \text { walking } \\ \text { (um)-sunod } & \text { pag-su-sunod } & \text { obeying } \\ \text { mag-laaral } & \text { pag-?a-2aaral } & \text { studying }\end{array}$

b. Occupational nouns

$\begin{array}{lll}\begin{array}{l}\text { Base form } \\ \text { (um)-tahi? }\end{array} & \begin{array}{l}\text { Reduplication form } \\ \text { ma-na-nahi? }\end{array} & \begin{array}{l}\text { Gloss } \\ \text { seamstress }\end{array}\end{array}$

c. $\quad$ Future aspect

$\begin{array}{lll}\begin{array}{l}\text { Base form } \\ \text { mag-liinis }\end{array} & \text { Reduplication form } & \text { Gloss } \\ \text { (um)-takboh } & \text { mag-lii-liinis } & \text { will clean } \\ & \text { (um)-taa-takboh } & \text { will run }\end{array}$

d. Causative adjectives

$\begin{array}{lll}\text { Base form } & \text { Reduplication form } & \text { Gloss } \\ \text { ?antok } & \text { na-kaa-ka-2antok } & \text { causing sleepiness }\end{array}$

e. Moderative verbs

$\begin{array}{lll}\text { Base form } & \text { Reduplication form } & \text { Gloss } \\ \text { mag-liinis } & \text { mag-liinis-liinis } & \text { clean a little } \\ \text { mag-walis } & \text { mag-walis-walis } & \text { sweep a little }\end{array}$

f. Intensive verbs

$\begin{array}{lll}\begin{array}{l}\text { Base form } \\ \text { mag-sugat }\end{array} & \begin{array}{l}\text { Reduplication form } \\ \text { (mag-)ka-sugat-sugat }\end{array} & \begin{array}{l}\text { Gloss } \\ \text { be thoroughly } \\ \text { covered with } \\ \text { wounds }\end{array} \\ & \end{array}$

This observation leads to the conclusion that either affixation or reduplication alone is often, if not always, insufficient in isolation to denote a morphosyntactic category. As depicted in (39), the selection of a particular affix and a particular shape of the reduplicant jointly determine a unique function, as emphasized by Lieber (1981:159-160). Tagalog thus presents very strong evidence that DME is real in natural languages because it is not redundant: two exponents are both indispensable for morphosyntactic disambiguation. 
(39)

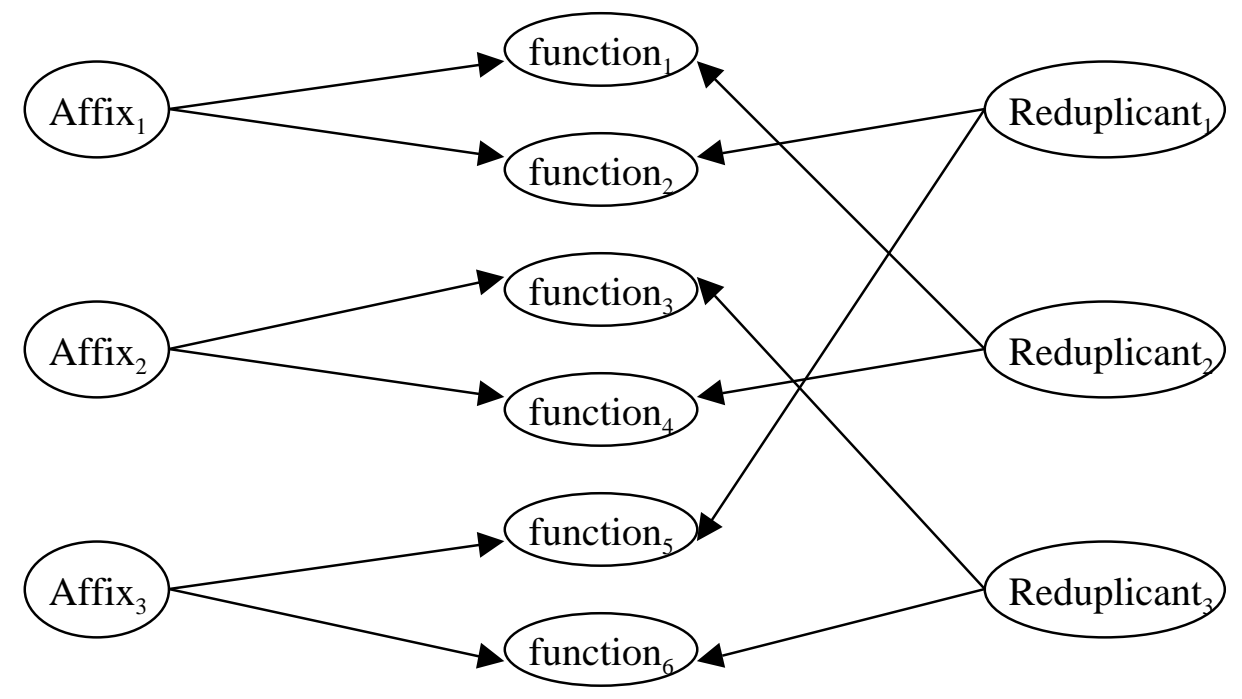

The analysis of DME in Tagalog should be straightforward at this point. By assumption, Integrity is violated by reduplication, so the relevant Faith-IO should be Integrity-IO. The sympathy candidate is a candidate which employs reduplication to fulfill RM but does not parse the underlying affixal material. Since the optimal candidate must mimic the property of the sympathy candidate, Max- $\mathrm{O}$ becomes crucial. This analysis was already presented in section 5.2, and the tableau is repeated in (40).

\begin{tabular}{|c|c|c|c|c|c|c|}
\hline & /mag-trabahoh/Future & $\operatorname{Max}$ & $\mathrm{RM}$ & $\begin{array}{c}\mathrm{Max} \\
\mathrm{O} \\
\end{array}$ & $\begin{array}{c}\text { Integrity } \\
\text { IO }\end{array}$ & $\begin{array}{l}\text { SPStem } \\
\equiv \text { PrWd }\end{array}$ \\
\hline a. & trabahoh & $* ! * *$ & $*$ & $* *$ & & \\
\hline b. & mag-trabahoh & & & $* ! *$ & & * \\
\hline c. & ta-trabahoh & $* ! * *$ & & & $* *$ & \\
\hline d. & mag-ta-trabahoh & & & & $* *$ & $*$ \\
\hline
\end{tabular}

Recall from section 5.2 that there is an important question which concerns Stem $\equiv$ PrWd: why does the sympathy candidate (40c) satisfy the selector constraint 
despite the fact that the left edge segment of the base does not occupy the left periphery of the entire prosodic word? This is not a question specific to Tagalog since Stem $\equiv$ PrWd is defined in such a way that no element other than a stem may be contained in a prosodic word. This indicates that the question is relevant in suffixal and infixal reduplication as well. I argued that the reason why the sympathy candidate satisfies Stem $\equiv \mathrm{PrWd}$ is captured by the assumption that IO-faithfulness constraints are satisfied if they are satisfied somewhere in the output, meaning that they do not have to be satisfied both in the base and in the reduplicant. This idea is the same as 'Broad IO-faithfulness' proposed by Struijke (1998). As schematically shown in (41a), the two reduplicative segments as well as the base-initial three segments are associated with the same base, and therefore, Anchor-L(Stem,PrWd) is satisfied by the reduplicant although it is violated in the base domain. By contrast, Anchor-R(Stem,PrWd) and Contiguity-Stem are violated by the reduplicant (because the base-medial [r] is skipped), but they are satisfied by the base. As encapsulated in (41b), all relevant faithfulness constraints are satisfied by the sympathy candidate in (40), and thus, Stem $\equiv P r W d$ is effectively fulfilled.

a. $\mathrm{trab}$ aho h

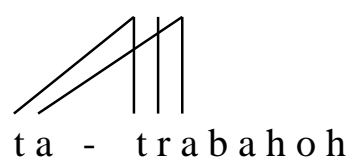

b.

\begin{tabular}{|l|c||c||c||}
\cline { 2 - 4 } \multicolumn{1}{l||}{} & $\begin{array}{c}\text { Anchor-L } \\
\text { (Stem,PrWd) }\end{array}$ & $\begin{array}{c}\text { Anchor-R } \\
\text { (Stem,PrWd) }\end{array}$ & $\begin{array}{c}\text { Contiguity- } \\
\text { Stem }\end{array}$ \\
\hline \hline Base & violated & satisfied & satisfied \\
\hline Reduplicant & satisfied & violated & violated \\
\hline Entire form & satisfied & satisfied & satisfied \\
\hline
\end{tabular}




\subsubsection{Emergence of the Unmarked in Reduplication}

The goal of this section is to present an analysis which determines the precise reduplicant shapes attested in Tagalog. Because Tagalog displays three types of reduplication as aforementioned and because different reduplicant shapes make a crucial contribution to disambiguate various morphosyntactic functions, it is essential that the grammar have a mechanism to derive the appropriate reduplicant shape. In this sense, the discussion developed in this section is tightly connected to DME in Tagalog although sympathy theory is irrelevant to the variation of reduplicant shapes. I argue that the variation of Tagalog reduplication is considered as the emergence of the unmarked derived from interactions of independently motivated constraints, as discussed by Spaelti (1997) and McCarthy and Prince (1999) among many others.

Tagalog has three kinds of reduplication: (i) initial CV of the base regardless the vowel length of the base-initial vowel, (ii) initial CVV where the first vowel of the base undergoes lengthening if it is short, and (iii) two syllables. Although the disyllabic reduplication examples in $(38 \mathrm{e}, \mathrm{f})$ appear to be total reduplication, disyllabic reduplication has another branch. In all the examples in (42) (Carrier 1984:293), the shape of the reduplicant is consistently CV(C)CVV, where the entire first syllable and the $\mathrm{CV}$ of the second syllable of the base are copied, the second vowel undergoing lengthening. The crucial difference between (38e, f) and (42) is the size of the base. When the base is disyllabic, the whole base is copied including the coda consonant of the second syllable. But the coda of the second syllable is not copied if the base is larger than two syllables (or more precisely, if the second syllable is not directly followed by a morpheme demarcation). Carrier $(1979,1984)$ regards (38e, f) and (42) as two sub-branches of a single reduplication type. 
Base form

tahiimik

baluktot

kalansin

?intindin
Reduplication form

tahii-tahiimik

baluu-baluktot

pagka-kalaa-kalansin

?intii-2intindin

Given this basic description of the three reduplicant shapes, the generalization is summarized in (43), where affixes are omitted to focus attention on reduplication. (43) shows that a certain morphosyntactic category is associated with a particular shape of the reduplicant. Although the earlier literature such as Carrier $(1979,1984)$, Lieber (1981), McCarthy (1981), and Marantz (1982) employs various templates to derive the right reduplicant shapes, I argue that the various templatic effects are obtained through constraint interactions a-templatically. Given CV-reduplication and CVV-reduplication, it is obvious that these reduplicant shapes are associated with something special to a given morphosyntactic category because vowel shortening and lengthening can be never obtained for purely phonological reasons in the same context. I demonstrate that the morpheme-peculiar nature is captured by relativizing morpheme-specific faithfulness constraints with respect to other constraints. This idea not only captures the contrast between CV-reduplication and CVV-reduplication but illuminates an integrated understanding of the entire pattern encapsulated in (43).

\begin{tabular}{|c|c|c|c|}
\hline Type & Morphological category & Reduplicant shape & Example \\
\hline (I) & $\begin{array}{c}\text { Gerunds } \\
\text { Occupational nouns }\end{array}$ & $\mathrm{CV}$ & $\begin{array}{c}\text { la-laakad } \\
\text { su-sunod } \\
\text { ka-kandilah } \\
\end{array}$ \\
\hline (II) & $\begin{array}{c}\text { Future aspect } \\
\text { Causative adjectives }\end{array}$ & CVV & $\begin{array}{l}\text { lii-liinis } \\
\text { taa-takboh } \\
\text { guu-gupit }\end{array}$ \\
\hline (III) & $\begin{array}{l}\text { Moderative verbs } \\
\text { Intensive verbs }\end{array}$ & $\begin{array}{c}\sigma \sigma \\
\sigma \mathrm{CVV}\end{array}$ & $\begin{array}{c}\text { walis-walis } \\
\text { baluu-baluktot }\end{array}$ \\
\hline
\end{tabular}


Considering the vowel length contrast between (43I) and (43II), vowel shortening occurs in the former while lengthening takes place in the latter. As a pertinent constraint which motivates vowel shortening, I assume *LongV (Rosenthall 1994). By contrast, the driving force of vowel lengthening has to do with stress facts in Tagalog. Schachter and Otanes (1972:16), Carrier (1979:118-119) and Soberano (1980:36) maintain that vowel length is contrastive in Tagalog. But French (1988) argues that $\mathrm{CVV}$ is not a legitimate syllable by investigating reduplication which is not relevant here. Rather, building on the observation that both primary stress and secondary stress cause phonetic vowel lengthening on a non-final syllable (French 1988:63) and that the syllable created by reduplication attracts foot-level (secondary) stress (French 1988:72), she proposes a stress-based vowel lengthening process in the language. In other words, her idea is the opposite of Schachter and Otane (1972), Carrier (1979), and Soberano's (1980) claim that stress is sensitive to vowel length, although syllable weight plays an important role, for example, in verb stress assignment (French 1988:71). Although this which-came-first-the-chicken-or-theegg question is an issue which has not received an unanimous solution, it is clear that vowel length and stress have a mutual correlation. This correlation is sufficient here.

As the driving force of vowel lengthening, I assume Align-R(RED, $\left.\sigma_{\mu \mu}\right)$, which maintains that the rightmost reduplicated syllable be heavy. A caveat is necessary. Although RED is contained as a variable, this should not be taken as the input morpheme. As I discussed in section 2.4, such phonologically empty but processspecific morphemes have no room to play a role in RMT. But the grammar must be still able to differentiate the base and the reduplicant since BR-correspondence constraints need to refer to them. Given the reduplication model assumed here (see 
section 2.2), the grammar's ability to recognize the base and the reduplicant is necessary for the computation of IO-correspondence constraints. Align-R(RED, $\left.\sigma_{\mu \mu}\right)$ is thus a markedness constraint. RED in Align- $R\left(R E D, \sigma_{\mu \mu}\right)$ is phonological substance of candidates produced by Gen but not a morpheme with serious theoretical status.

Another background concerns the fact that the reduplicant is maximally disyllabic. Spaelti (1997) discusses that the size of the reduplicant can be properly adjusted by alignment constraints. Crucially, Align-L(Ft,PrWd) » Max-BR yields foot size reduplication, and Align-L $(\sigma, \operatorname{PrWd}) »$ Max-BR monosyllabic one. I take advantage of these alignment constraints as size restrictors, henceforth abbreviating them as AllFtL and AlloL respectively. Given that only two syllables are maximally reduplicated, AllFtL is undominated in the base-reduplicant dimension. The base is not subject to the restriction imposed by the restrictors. This shows that Max-IO dominates them. Given this, we can establish a partial constraint ranking in (44). This ranking yields a mini-typology by placing Max-BR in various slots: (i) AlloL » Max-BR generates a monosyllabic reduplicant, (ii) AllFtL » Max-BR » All $\sigma \mathrm{L}$ provides a foot size reduplicant, and (iii) Max-BR » AllFtL yields total reduplication, modulo no other factor enters the picture. Because the reduplicant is monosyllabic or disyllabic in Tagalog, only the first two options are available in the language.

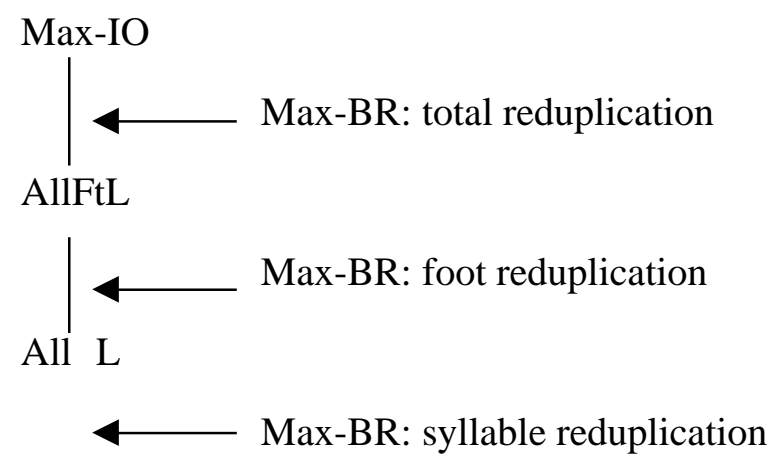


Beginning with (43I), All $\sigma \mathrm{L} »$ Max-BR is not sufficient, taking [ka-kandilah]. Two serious competitors need to be considered against the optimal form: [kaakandilah] and [kan-kandilah]. The optimal form violates Align-R(RED, $\left.\sigma_{\mu \mu}\right)$, so *LongV must be ranked over it to rule out [kaa-kandilah]. Second, I employ NoCoda as the pertinent constraint penalizing [kan-kandilah]. As demonstrated in (45), this analysis properly restricts the size of the reduplicant to $\mathrm{CV}$. The three high ranked constraints prohibit any deviance from CV-reduplication. The same analysis holds of cases where the base-initial syllable contains a long vowel, as illustrated in (46). In the subseuqnet tableaux in this section, violations of AlloL are indicated numerically.

\begin{tabular}{|c|c|c|c|c|c|c|}
\hline & /kandilah/Type (I) & $\begin{array}{c}\text { No } \\
\text { Coda }\end{array}$ & All $\sigma \mathrm{L}$ & $\begin{array}{c}* \text { Long } \\
\mathrm{V} \\
\end{array}$ & $\begin{array}{c}\text { Max } \\
\text { BR }\end{array}$ & $\begin{array}{c}\text { Align-R } \\
\left(\mathrm{RED}, \sigma_{\mu \mu}\right)\end{array}$ \\
\hline a. & ka-kan.di.lah & $* *$ & 6 & & $* * * * * *$ & $*$ \\
\hline b. & kaa-kan.di.lah & $* *$ & 6 & $* !$ & $* * * * * *$ & \\
\hline c. & kan-kan.di.lah & $* * * !$ & 6 & & $* * * * *$ & \\
\hline $\mathrm{d}$. & kaa.di-kan.di.lah & $* *$ & $10 !$ & $*$ & $* * * *$ & $*$ \\
\hline e. & kan.di-kan.di.lah & $* * * !$ & 10 & & $* * *$ & $*$ \\
\hline
\end{tabular}

\begin{tabular}{|c|c|c|c|c|c|c|}
\hline & /laakad/Type (I) & $\begin{array}{c}\text { No } \\
\text { Coda }\end{array}$ & All $\sigma \mathrm{L}$ & $\begin{array}{c}\text { *Long } \\
\mathrm{V} \\
\end{array}$ & $\begin{array}{c}\mathrm{Max} \\
\mathrm{BR} \\
\end{array}$ & $\begin{array}{c}\text { Align-R } \\
\left(\mathrm{RED}, \sigma_{\mu \mu}\right)\end{array}$ \\
\hline a. & laa-laa.kad & $*$ & 3 & $* * !$ & $* * *$ & \\
\hline b. & la-laa.kad & $*$ & 3 & $*$ & $* * *$ & $*$ \\
\hline c. & laa.kad-laa.kad & $* * !$ & 6 & $* *$ & & \\
\hline d. & la.kad-laa.kad & $* * !$ & 6 & $*$ & & \\
\hline e. & la.ka-laa.kad & * & $6 !$ & * & $*$ & * \\
\hline f. & la.kaa-laa.kad & $*$ & $6 !$ & $* *$ & $*$ & \\
\hline
\end{tabular}


Turning to CVV-reduplication in (43II), Max-BR and Align-R(RED, $\left.\sigma_{\mu \mu}\right)$ still need to be ranked beneath All $\sigma \mathrm{L}$, but they must outrank *LongV, yielding Max-BR, Align-R $\left(\mathrm{RED}, \sigma_{\mu \mu}\right) » *$ LongV. This obtains the effect of CVV reduplication. As illustrated in (47) and (48), CVV-reduplication surfaces irrespective of the vowel length of the base-initial syllable. Two important remarks are in order. First, Max$\mathrm{BR}$ and Align-R(RED, $\left.\sigma_{\mu \mu}\right)$ must be jointly reranked with respect to *LongV to explain the contrast between (43I) and (43II). Reranking of * LongV with only either Max-BR or Align-R(RED, $\left.\sigma_{\mu \mu}\right)$ cannot capture the whole range of patterns in (43I) and (43II). Second, this reranking is conceptualized in terms of morphosyntactic markings on Max-BR. This idea is consistent with one of the central proposals in this work that morpheme-specific faithfulness constraints may be relativized with respect to other constraints. Since gerunds and occupational nouns belong to type (I), long vowels are prohibited in the reduplicant as in (45) and (46), while possessing a heavy syllable in the reduplicant is more important in type (II) morphosyntactic categories such as the future aspect and causative adjectives. Given high ranked NoCoda, vowel lengthening is required when the base-initial vowel is short, as demonstrated in (48). The different rankings of Max-BR and Align-R $\left(\mathrm{RED}, \sigma_{\mu \mu}\right)$ with respect to $*$ LongV account for the contrast between type (I) and type (II).

\begin{tabular}{|ll||c|c|c|c|c|}
\hline & \multicolumn{1}{|c||}{$/$ liinis/ $/$ Type (II) } & $\begin{array}{c}\text { No } \\
\text { Coda }\end{array}$ & All $\sigma \mathrm{L}$ & $\begin{array}{c}\text { Max } \\
\text { BR }\end{array}$ & $\begin{array}{c}\text { Align-R } \\
\left(\mathrm{RED}, \sigma_{\mu \nu}\right)\end{array}$ & $\begin{array}{c}* \text { Long } \\
\mathrm{V}\end{array}$ \\
\hline \hline a. & lii-lii.nis & $*$ & 3 & $* * *$ & & $* *$ \\
\hline b. & li-lii.nis & $*$ & 3 & $* * *$ & $* !$ & $*$ \\
\hline c. & lii.ni-lii.nis & $*$ & $6 !$ & $*$ & $*$ & $* *$ \\
\hline d. & lii.nis-lii.nis & $* * !$ & 6 & & & $* *$ \\
\hline
\end{tabular}




\begin{tabular}{|ll||c|c|c|c|c|}
\hline & \multicolumn{1}{|c||}{ gupit/Type (II) } & $\begin{array}{c}\text { No } \\
\text { Coda }\end{array}$ & All $\sigma \mathrm{L}$ & $\begin{array}{c}\text { Max } \\
\text { BR }\end{array}$ & $\begin{array}{c}\text { Align-R } \\
\left(\mathrm{RED}, \sigma_{u \mu}\right)\end{array}$ & $\begin{array}{c}* \text { Long } \\
\text { V }\end{array}$ \\
\hline \hline a. & gu-gu.pit & $*$ & 3 & $* * *$ & $* !$ & \\
\hline b. & guu-gu.pit & $*$ & 3 & $* * *$ & & $*$ \\
\hline c. & gup-gu.pit & $* * !$ & 3 & $* *$ & & \\
\hline d. & gu.pi-gu.pit & $*$ & $6 !$ & $*$ & $*$ & \\
\hline e. & gu.pit-gu.pit & $* * !$ & 6 & & & \\
\hline
\end{tabular}

Finally, consider (43III). It is obvious that the block constraints (i.e., Max-BR and Align-R $\left.\left(\mathrm{RED}, \sigma_{\mu \mu}\right)\right)$ must be ranked between AllFtL and AlloL in this case. The question is how the different behaviors of coda and vowel lengthening attested between disyllabic and longer bases can be well captured. I demonstrate that this string dependency is indeed understood as an emergence of the unmarked effect. Cases where the base is longer than two syllables follow rather straightforwardly given the discussion so far: Max-BR and Align-R(RED, $\left.\sigma_{\mu \mu}\right)$ intervene between NoCoda and All $\sigma \mathrm{L}$. As illustrated in (49), NoCoda ranked over Max-BR and Align$\mathrm{R}\left(\mathrm{RED}, \sigma_{\mu \mu}\right)$ prohibits the copy of the coda consonant in the base-second syllable, but these constraints ranked above All $\sigma \mathrm{L}$ and $*$ LongV require disyllabic reduplication and vowel lengthening. Moreover, Align-R(RED, $\left.\sigma_{\mu \mu}\right)$ plays an important role here. Given that vowel lengthening occurs in the syllable abutting on the left edge of the base-initial syllable, it is not sufficient to establish a constraint that demands the reduplicant to carry a heavy syllable. A potential but undesirable candidate (49f) cannot be eliminated by such a constraint, but it is successfully ruled out by Align$\mathrm{R}\left(\mathrm{RED}, \sigma_{\mu \mu}\right)$ since it specifically requires the right edge of the reduplicant to carry a heavy syllable (compare (49d) and (49f)). 


\begin{tabular}{|lc||c|c|c|c|c|}
\hline & \multicolumn{1}{|c||}{ baluktot/Type (III) } & $\begin{array}{c}\text { No } \\
\text { Coda }\end{array}$ & $\begin{array}{c}\text { Max } \\
\text { BR }\end{array}$ & $\begin{array}{c}\text { Align-R } \\
\left(\mathrm{RED}, \sigma_{\mu \mathrm{u}}\right)\end{array}$ & $\begin{array}{c}\text { All } \sigma \mathrm{L} \\
* \text { Long } \\
\mathrm{V}\end{array}$ \\
\hline \hline a. & ba-ba.luk.tot & $* *$ & $* * * * * ! *$ & $*$ & 6 & \\
\hline b. & baa-ba.luk.tot & $* *$ & $* * * * * ! *$ & & 6 & $*$ \\
\hline c. & ba.lu-ba.luk.tot & $* *$ & $* * * *$ & $* !$ & 10 & \\
\hline d. & ba.luu-ba.luk.tot & $* *$ & $* * * *$ & & 10 & $*$ \\
\hline e. & ba.luk-ba.luk.tot & $* * * !$ & $* * *$ & & 10 & \\
\hline f. & baa.lu-ba.luk.tot & $* *$ & $* * * *$ & $* !$ & 10 & $*$ \\
\hline
\end{tabular}

The same ranking makes a wrong prediction for cases where the base is disyllabic, however, because the base-final consonant would not be copied, contrary to fact. My proposal is that the ranking in (49) can be retained but another crucial constraint must be introduced: Hierarchical Anchor-BR (HierAnch-BR) which requires that both edges of the reduplicant be anchored at the base. Expressed differently, this constraint demands total reduplication. If HierAnch-BR is ranked over NoCoda, the right result obtains, as exemplified in (50).

\begin{tabular}{|lc||c|c|c|c|}
\hline & \multicolumn{1}{|c|}{$/$ walis/Type (III) } & $\begin{array}{c}\text { HierAnch- } \\
\text { BR }\end{array}$ & NoCoda & Max-BR & $\begin{array}{c}\text { Align-R } \\
\left(\mathrm{RED}, \sigma_{\mu \mu}\right)\end{array}$ \\
\hline a. & wa-wa.lis & $* !$ & $*$ & $* * *$ & $*$ \\
\hline b. & waa-wa.lis & $* !$ & $*$ & $* * *$ & \\
\hline c. & wa.li-wa.lis & $* !$ & $*$ & $*$ & $*$ \\
\hline d. & wa.lii-wa.lis & $* !$ & $*$ & $*$ & \\
\hline e. $\quad$ wa.lis-wa.lis & & $* *$ & & \\
\hline
\end{tabular}

The introduction of HierAnch-BR does no harm to cases where the base is larger than two syllables. Suppose that AllFtL outranks HierAnch-BR. The 
reduplicant must be partial in those cases, so HierAnch-BR is necessarily violated to satisfy higher ranked AllFtL. This indicates that HierAnch-BR plays no decisive role in (49), and therefore, the evaluation in (49) still holds. HierAnch-BR cannot occupy a fixed position, however. Taking (43I) and (43II) into consideration, HierAnch-BR should not be ranked over All $\sigma \mathrm{L}$ because total reduplication is expected otherwise when the base is disyllabic: AllFtL is vacuously satisfied in such cases, so HierAnchBR enjoys a decisive role. HierAnch-BR is also a faithfulness constraint, so it is also relativized with respect to various morphosyntactic categories. For (43I) and (43II), HierAnch-BR is ranked below All $\sigma \mathrm{L}$ to avoid total reduplication of disyllabic bases.

Summarizing the analysis above, the whole constraint ranking is given in (51). The three patterns of Tagalog reduplication are emergence of the unmarked effects. But the degree of unmarkedness of the reduplicant is different from morphosyntactic category to category. Given the ranking in (51), CV-reduplication is the least marked and disyllabic reduplication is the most marked among the three types.

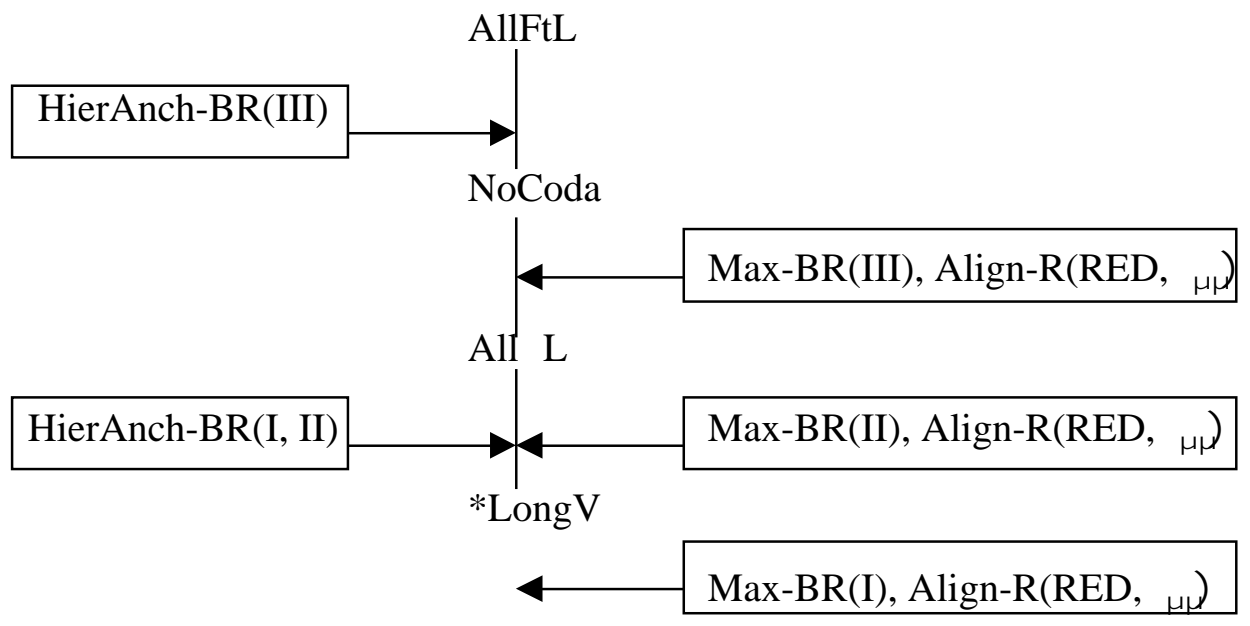

Integrating the analysis here with the discussion in the preceding section, a given morpheme subcategorizes a particular affix, but affixation is not enough to 
satisfy RM due to the presence of the selector constraint (i.e., Stem $\equiv$ PrWd). Given the $\mathrm{RM} \gg$ Integrity-IO ranking, reduplication is recruited as a secondary realization of the morpheme. The shape of the reduplicant depends on the position of the relevant faithfulness constraints.

The relativization of Max-BR, Align-R(RED, $\left.\sigma_{\mu \mu}\right)$ and HierAnch-BR poses an interesting question regarding the status of RED as a real morpheme. Given that these constraints are essentially sensitive to the distinction between the base and the reduplicant, does this imply the existence of RED in the input such that this morpheme functions as the imperative to derive reduplication? The answer is negative. I have proposed that all faithfulness constraints are subdivided into subconstraints, each of which carries a morphosyntactic marking. The reason why the relativization of Max-BR and HierAnch-BR are tangible in Tagalog morphology is that morphological opacity enters the grammar and Integrity-IO is ranked below RM. But the grammar still needs to be able to make the distinction between the base and the reduplicant since all BR-faithfulness constraints would not be able to be computed otherwise. Decomposition of BR-faithfulness constraints therefore does not presuppose the existence of the imperative morpheme in the underlying representation. This conforms to the idea developed in this work.

\subsection{Phonological Polarity}

In this section, I briefly discuss phonological polarity as a final case study of DME, taking the plural formation in Luo as a representative example (Stafford 1967; Gregersen 1972; Okoth-Okombo 1982; Stonham 1994). As reviewed in section 2.5, it constitutes an important empirical phenomenon taken to be support for anti- 
faithfulness theory by Alderete (1999). The goal of this section is to demonstrate that the sympathy-based idea of morphological opacity successfully explains the phonological polarity effect in Luo. As a consequence, I argue that anti-faithfulness theory is not the only available analytical possibility in OT.

Relevant data are repeated in (52). Again, the descriptive generalization is as follows: (i) a plural suffix $-i$ or $-e$ (phonetically realized either as $[\mathrm{e}]$ or as $[\varepsilon]$ ) is attached, (ii) a word-final vowel of a singular form is subject to deletion, and (iii) the voicing value of the final consonant is reversed (i.e., [-voi] $\rightarrow[+$ voi $]$, and vice versa). Under the system developed in chapter 2, morphosyntactic functions are not inherent properties of stems and therefore, the singular and the plural are derived by assigning the singular and the plural morphemes to bare stems respectively. Because singular forms are phonologically identical to (the outputs of) bare stems in Luo, this point does not have any serious repercussion on the following discussion.

$$
\text { Singular Plural Gloss }
$$

\begin{tabular}{|c|c|c|}
\hline alot & alode & vegetable(s) \\
\hline bat & bade & $\operatorname{arm}(\mathrm{s})$ \\
\hline $\operatorname{lu} \theta$ & luð $\varepsilon$ & stick(s) \\
\hline $10 \theta$ & ruoői & chief(s) \\
\hline uok & guogi & $\operatorname{dog}(s)$ \\
\hline
\end{tabular}

$\begin{array}{llll}\text { b. } & \text { kidi } & \text { kite } & \text { stone(s) } \\ \text { puoðo } & \text { puoøe } & \text { garden(s) } \\ \text { got } & \text { gode } & \text { twig(s) } \\ \text { cogo } & \text { coke } & \text { bone(s) } \\ \text { deb } \varepsilon & \text { dep } \varepsilon & \text { debbi(s) }\end{array}$

Given that the plural forms undergo a voicing change besides affixation, the plural formation can be considered as an instance of DME. Building upon the argument in this chapter, the reason why the voicing polarity is required is that the suffix $-i$ or $-e$ becomes morphologically opaque due to the existence of Stem $\equiv \mathrm{PrWd}$ 
serving as the selector constraint. As shown in (53) and (54), the voicing alternation can be accounted for in parallel to other cases of DME examined above. In (52b), the stem-final vowel is subject to deletion. I assume that this is because of high ranking *Hiatus. In the two tableaux below, I attach the plural marking on Max to ensure that the affixal vowel remains undeleted.

\begin{tabular}{|c|c|c|c|c|c|c|c|}
\hline & & /alot-e/ Plural & $\operatorname{Max}_{\text {Plural }}$ & $\mathrm{RM}$ & $\begin{array}{c}\text { Ident } \\
\text { O-[voi] }\end{array}$ & $\begin{array}{c}\text { Ident } \\
\text { IO-[voi] }\end{array}$ & $\begin{array}{l}\text { S Stem } \\
\equiv \text { PrWd } \\
\end{array}$ \\
\hline a. & & alot & $* !$ & $*$ & $*$ & & \\
\hline b. & & alote & & & $* !$ & & $*$ \\
\hline c. & 89 & alod & $* !$ & & & $*$ & \\
\hline d. & 嘫 & alode & & & & $*$ & * \\
\hline
\end{tabular}

\begin{tabular}{|c|c|c|c|c|c|c|}
\hline & /kidi-e/Plural & $\operatorname{Max}_{\text {Plural }}$ & $\mathrm{RM}$ & $\begin{array}{c}\text { Ident } \\
\text { O-[voi] }\end{array}$ & $\begin{array}{c}\text { Ident } \\
\text { IO-[voi] }\end{array}$ & 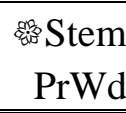 \\
\hline a. & kidi & $* !$ & $*$ & $*$ & & \\
\hline b. & kide & & & $* !$ & & $*$ \\
\hline c. & kiti & $* !$ & & & $*$ & \\
\hline d. $\quad$ 吗 & kite & & & & $*$ & $*$ \\
\hline
\end{tabular}

(53) and (54) show that anti-faithfulness theory is not the necessary tool to analyze phonological polarity phenomena. Since some stem modification is involved in addition to affixation, the voicing exchange in the plural formation in Luo is successfully subsumed under the theme of DME. Given that phonological polarity is also driven by RM in the sense that some stem modification is required because of morphological opacity of the affixal element, the analysis here captures the intuition that the voicing exchange is morphologically motivated. One might claim that the 
proposed analysis can derive the morphological derivedness simply by tagging a morphosyntactic category to a faithfulness constraint. This is not a valid counterargument, however, since morphological idiosyncrasy must be incorporated in any analysis.

In anti-faithfulness theory espoused by Alderete (1999), this morphological character cannot be captured. As pointed out in section 2.5, the theory needs to stipulate that anti-faithfulness constraints are operative only in the surface-to-surface (or output-output) dimension, but even this stipulation does not explain why the voicing polarity is morphologically governed since anti-faithfulness constraints are potentially able to produce phonological alternations with no morphological reasons. Put differently, how can we prevent $\neg$ Faith-IO » Faith-IO in a firmly grounded way? Since a given alternation is not forced by a markedness constraint, it should not be phonological. The only remaining possibility is that it is morphologically conditioned, but there is no principled way to prevent the morpheme-free activation of $\neg$ Faith-IO. The existence of $\neg$ Faith-IO generates context-free anti-faithfulness effects, contrary to fact. Anti-faithfulness effects are restricted to cases where a morpheme needs to receive phonological exponence. To ensure the desired effect, an additional assumption is necessary: anti-faithfulness constraints are activated by morphemes, as explicitly done by Alderete (1999). This proves that morphosyntactic information needs to be encoded as part of faithfulness constraints in anti-faithfulness theory as well, showing that the idea of attaching a morphosyntactic marking does not constitute a damaging argument for RMT developed throughout this dissertation. The fact that phonological polarity is induced by the introduction of a new morpheme is directly captured by RMT but not by anti-faithfulness theory. 


\subsection{Theoretical Predictions}

I have proposed a general schema to explain DME and applied it to various concrete cases. This section addresses theoretical predictions of the sympathy-based system. The discussion is centered on theoretical restrictiveness of the sympathy mechanism, particularly concerning possible and impossible morphological constructions in natural languages. I also compare RMT with anti-faithfulness theory in this context.

I start the discussion with the most important prediction of the sympathy system. DME can be more generally regarded as multiple morphemic exponence. However, it is never the case that natural languages are free from the restriction on the number of phonological exponence associated with a single morpheme. The range is quite limited: minimally zero (i.e., no morphemic exponence at the cost of a RM violation) and maximally two. An exhaustive survey of all human languages is beyond anyone's capacity, but there is no counterexample to this generalization to the best of my knowledge. The formal prediction discussed here is concerned with this generalization, especially the upper bound of morphemic exponence in languages.

Consider the following schematic example: $/ \mathrm{ABC}-\mathrm{D}_{\alpha} / \alpha$, where $A B C$ are stem segments, and the suffixal element $D$ contributes to the realization of morpheme $\alpha$ if it has some correspondent in the output. Another morphological expression of $\alpha$ is obtained if some stem modification takes place, resulting in DME (e.g., [AB'CD] or $\left.\left[A B C^{\prime} D\right]\right)$. The question is then whether an additional stem change is possible (e.g., $\left.\left[A B^{\prime} C^{\prime} D\right]\right)$, exhibiting triple morphemic exponence. Given the theoretical mechanism articulated here, this is an impossible state of affairs. Consider the tableau in (55). In order to obtain two stem changes, it must be minimally true that RM outranks two faithfulness constraints. But the violations incurred by (55b) and (55c) constitute 
only a subset of those incurred by (55d), so (55d) is harmonically bounded by (55b) and (55c) in the sense of Prince and Smolensky (1993:176-178): no ranking permutation makes (55d) better than (55b) and (55c). This point proves that no sympathetic correspondence can make (55d) optimal because the selector constraint chooses either $\left[\mathrm{AB}^{\prime} \mathrm{C}\right]$ or $\left[\mathrm{ABC} \mathrm{C}^{\prime}\right]$ as the operative sympathy candidate depending on the relative hierarchy of Faith-B and Faith-C. An immediate consequence is that either (55f) or $(55 \mathrm{~g})$ is the final output: $(55 \mathrm{~h})$ is harmonically bounded by them. This shows that more than two phonological exponents of a single morpheme are never obtained mechanically within the sympathy system.

\begin{tabular}{|c|c|c|c|c|c|c|c|}
\hline & & $/ \mathrm{ABC}-\mathrm{D}_{\alpha} /{ }_{\alpha}$ & Max & RM & Faith-B & Faith-C & Stem $\equiv \operatorname{PrWd}$ \\
\hline a. & & $\mathrm{ABC}$ & $* !$ & $*$ & & & \\
\hline b. & 8 & $A B C^{\prime}$ & $* !$ & & & $*$ & \\
\hline c. & 8 & $\mathrm{AB}^{\prime} \mathrm{C}$ & $* !$ & & $*$ & & \\
\hline d. & & $\mathrm{AB}^{\prime} \mathrm{C}^{\prime}$ & $* !$ & & * & $*$ & \\
\hline e. & & $\mathrm{ABCD}$ & & & & & * \\
\hline f. & 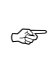 & $A B C^{\prime} D$ & & & & $*$ & $*$ \\
\hline g. & 唱 & $\mathrm{AB}^{\prime} \mathrm{CD}$ & & & $*$ & & $*$ \\
\hline h. & & $A B^{\prime} C^{\prime} D$ & & & $*$ & $*$ & $*$ \\
\hline
\end{tabular}

This point has a significant correlation with a general property of nonconcatenative morphological operations discussed in section 3.2.1. I argued that it is mechanically impossible in RMT that subtractive morphology and metathesis, for example, cooccur if they are both morphologically conditioned. To recall this point, consider a schematic example: $/ \mathrm{ABC}_{\alpha}$. Morpheme $\alpha$ does not bear any phonological 
content underlyingly. Depending on the specific faithfulness constraint ranked beneath RM, the concrete stem modification is determined. As (56) shows, the candidate with two stem changes is harmonically bounded by the candidates with one stem change. This argument suggests that maximally one phonological exponent can appear when no affix is present. Thus, no language can exist which displays both umlaut and metathesis in order to obtain phonological exponence of a morpheme, for example.

\begin{tabular}{|c|c|c|c|c|c|}
\hline & & $/\left.\mathrm{ABC}\right|_{\alpha}$ & $\mathrm{RM}$ & Faith-B & Faith-C \\
\hline a. & & $\mathrm{ABC}$ & $* !$ & & \\
\hline b. & 电 & $\mathrm{ABC}^{\prime}$ & & & $*$ \\
\hline c. & 四 & $\mathrm{AB}^{\prime} \mathrm{C}$ & & $*$ & \\
\hline $\mathrm{d}$. & & $\mathrm{AB}^{\prime} \mathrm{C}^{\prime}$ & & $*$ & $*$ \\
\hline
\end{tabular}

This has a direct repercussion on the upper limit of morphemic exponence predicted by the sympathy account. Since the selector constraint (i.e., Stem $\equiv$ PrWd) simply makes the existence of affixes invisible, DME cases cannot deviate from simple examples of nonconcatenative morphology concerning the restriction imposed on stem changes. DME is a hybrid of concatenative and nonconcatenative morphology. DME is nothing different from examples discussed in chapter 2 except that some affix exists.

In summary, the whole argument so far indicates that the theoretical system developed in this work makes the following two important interrelated predictions. First, DME is possible only when an affix exists in the underlying representation. Second, more than two phonological expressions are never permitted in any languages. Every language has its own phonology, so various phonological changes 
can occur on a stem. But some phonological factor must come into play when more than one stem change is found. The plural formation in German stated at the outset of this chapter is a clear example. In Buch $\approx$ Bücher, the stem-final consonant exhibits the $[\mathrm{x}] \approx[\mathrm{c}]$ alternation. The plural form is different from the output of the bare stem (which is phonologically identical to the singular form) in three respects (i.e., -er, umlaut, and $[\mathrm{x}] \rightarrow[c ̧])$. But the distribution of $[\mathrm{x}]$ and $[\mathrm{c}]$ is quite regular and predictable in German (i.e., [x] after a back vowel, and [ç] elsewhere). The $[\mathrm{x}] \approx[c ̧]$ alternation is a phonological consequence of umlaut, and therefore, the segmental alternation is motivated by a high ranked markedness constraint. Given that this alternation is phonologically conditioned, the $[\mathrm{x}] \approx[\mathrm{c}]$ alternation is not an exponent of the plural morpheme.

Consider anti-faithfulness theory in terms of these theoretical predictions. Taking Gast $\approx$ Gäste 'guest(s)' in German as an example (see (1) for more examples), the plural formation is analyzed under anti-faithfulness theory that the plural affix $-e$ activates $\neg$ Ident-IO-[+back]. This anti-faithfulness constraint is obviously ranked over the faithfulness counterpart, namely Ident-IO-[+back], as shown in (57).

\begin{tabular}{|ll||c|c|c|}
\hline & \multicolumn{1}{|c|}{$/$ Gast-e/ Plural } & Max & ᄀIdent-IO-[+back] & Ident-IO-[+back] \\
\hline \hline a. & Gast & $* !$ & $*$ & \\
\hline b. & Gaste & & $* !$ & \\
\hline c. & Gäst & $* !$ & & $*$ \\
\hline d. & Gäste & & & $*$ \\
\hline
\end{tabular}

Despite the fact that anti-faithfulness theory works, it makes a prediction different from the sympathy-based system in terms of restrictiveness of possible nonconcatenative morphology in natural languages. The anti-faithfulness account 
does not restrict the number of changes caused on a stem since multiple antifaithfulness constraints can dominate the corresponding faithfulness constraints. Suppose that $\neg$ Ident-IO-[+back], $\neg$ Integrity-IO and $\neg$ Linearity-IO outrank Ident-IO[+back], Integrity-IO and Linearity-IO respectively. The optimal output form is required to undergo umlaut, reduplication and metathesis in this scenario, but no such real case would exist. Thus, the anti-faithfulness account suffers from an overgeneration problem. Functionally, one might claim that triple or more phonological exponence of a morpheme is blocked in terms of redundancy. But DME is also redundant in the first place in languages like German and Japanese because the absence of umlaut in German plurals or accentual changes in Japanese would not reveal a different morphosyntactic function. The theoretical predictions discussed above are empirically correct to the best of my knowledge. Although they should be subject to further empirical scrutiny, it is certainly unlikely that a single morpheme must receive ten phonological exponents, as predicted to be possible by antifaithfulness theory.

In the context of DME, Stem $\equiv$ PrWd is the only relevant constraint. According to McCarthy (1999), where sympathy theory was originally proposed, any faithfulness constraint can potentially serve as a selector constraint. It is thus necessary here to demonstrate that DME is not obtained no matter what other faithfulness constraints are arbitrarily chosen as the selector constraint. Since the following argument applies to any faithfulness constraint, I arbitrarily choose Linearity as the selector constraint for the test case here. Suppose that $/ A B C-D_{\alpha} / \alpha$ is given as the underlying representation and that Linearity-IO is the selector constraint. As delineated in (58), the candidate faithful to the underlying representation (i.e., 
(58e)) is chosen as the sympathy candidate because it does not incur any constraint violation. This shows that (58e) is the winning form regardless of the specific FaithO to be posited. (58e) has only one exponent of morpheme $\alpha$, and therefore, DME does not appear. The reason is simply that no faithfulness constraint other than Stem $\equiv$ PrWd can make the affixal element opaque, and as a result, that there is no reason to deform the phonological shape of the base to satisfy RM.

\begin{tabular}{|c|c|c|c|c|c|c|}
\hline & & $/ \mathrm{ABC}-\mathrm{D}_{\alpha} /_{\alpha}$ & Max & $\mathrm{RM}$ & Faith-IO-[C] & SLinearity \\
\hline a. & & $\mathrm{ABC}$ & $* !$ & $*$ & & \\
\hline b. & & $\mathrm{ABC}^{\prime}$ & $* !$ & & $*$ & \\
\hline c. & & BAC & $* !$ & & & $*$ \\
\hline d. & & $\mathrm{BAC}^{\prime}$ & $* !$ & & $*$ & $*$ \\
\hline & 的四 & $\mathrm{ABCD}$ & & & & \\
\hline f. & & $A B C^{\prime} D$ & & & $* !$ & \\
\hline g. & & BACD & & & & $* !$ \\
\hline h. & & $B A C^{\prime} D$ & & & $* !$ & $*$ \\
\hline
\end{tabular}

One might bring up the possibility of multiple sympathy. This possibility has two ramifications: (i) Stem $\equiv \mathrm{PrWd}$ and some other faithfulness constraint are selector constraints, and (ii) faithfulness constraints other than Stem $\equiv$ PrWd serve as the selector constraints. I begin with case (i). Consider the tableau in (59), where Stem $\equiv \operatorname{PrWd}$ and Faith-IO-[C] are selector constraints. The principal question here is whether (59h) has ever a chance to surface as the best output. In (59), three candidates are indicated by the flower mark due to the fact that more than one selector constraint is employed here. For ${ }^{8}$ Faith-IO-[C], (59e) is selected as the 
sympathy candidate whereas (59b) or (59c) is the sympathy candidate for Stem $=$ PrWd. The selection between (59b) and (59c) depends on the ranking of Faith-IO-[B] and Faith-IO-[C]. Given these sympathy candidates, (59h) has no chance to be selected as the ultimate output. First, whatever Faith- $\mathrm{O}$ constraint is chosen through which (59e) is sympathized, (59h) is harmonically bounded by (59e) given that the violations of (59e) are a subset of those incurred by (59h). Second, for (59b) or (59c) to be active as a sympathy candidate, either Faith-80 O-[C'] or FaithO O-[B'] should be the sympathy constraint. Either (59f) or (59g) fares better than (59h) by the harmonic bounding reasoning. This general consideration indicates that triple morphemic exponence is never obtained even if some faithfulness constraint is active as a selector constraint in addition to Stem $\equiv \mathrm{PrWd}$.

\begin{tabular}{|c|c|c|c|c|c|c|}
\hline & $/ \mathrm{ABC}-\mathrm{D}_{\alpha} / \alpha$ & Max & $\mathrm{RM}$ & $\begin{array}{c}\text { Faith } \\
\text { IO-[B] }\end{array}$ & $\begin{array}{l}\text { IO-[C] } \\
\text { IOAth }\end{array}$ & $\begin{array}{l}\text { Stem } \\
\equiv \text { PrWd }\end{array}$ \\
\hline a. & $\mathrm{ABC}$ & $* !$ & $*$ & & & \\
\hline b. & $\mathrm{ABC}^{\prime}$ & $* !$ & & & * & \\
\hline c. & $\mathrm{AB}^{\prime} \mathrm{C}$ & $* !$ & & $*$ & & \\
\hline d. & $\mathrm{AB}^{\prime} \mathrm{C}^{\prime}$ & $* !$ & & * & * & \\
\hline e. & $\mathrm{ABCD}$ & & & & & * \\
\hline f. & $A B C^{\prime} D$ & & & & $*$ & $*$ \\
\hline g. & $\mathrm{AB}^{\prime} \mathrm{CD}$ & & & $*$ & & $*$ \\
\hline h. & $A B^{\prime} C^{\prime} D$ & & & $*$ & $*$ & $*$ \\
\hline
\end{tabular}

Let us turn to case (ii), where multiple selector constraints are both not Stem $\equiv$ PrWd. As shown in (60), this case is even simpler. Since the phonological material affiliated with the affix is not required to be underparsed when Stem $\equiv P r W d$ 
is not in force as a selector constraint, complete faithfulness is the best. (60e) incurs no violation of the relevant constraints, and therefore, it is chosen as the sympathy candidate regardless of the relative ranking of Faith-IO-[B] and Faith-IO-[C]. The immediate result is that $(60 \mathrm{e})$ surfaces irrespective of the particular Faith- 80 constraint. We can thus conclude that more than one phonological exponent never appears when Stem $\equiv$ PrWd is not employed as the selector constraint.

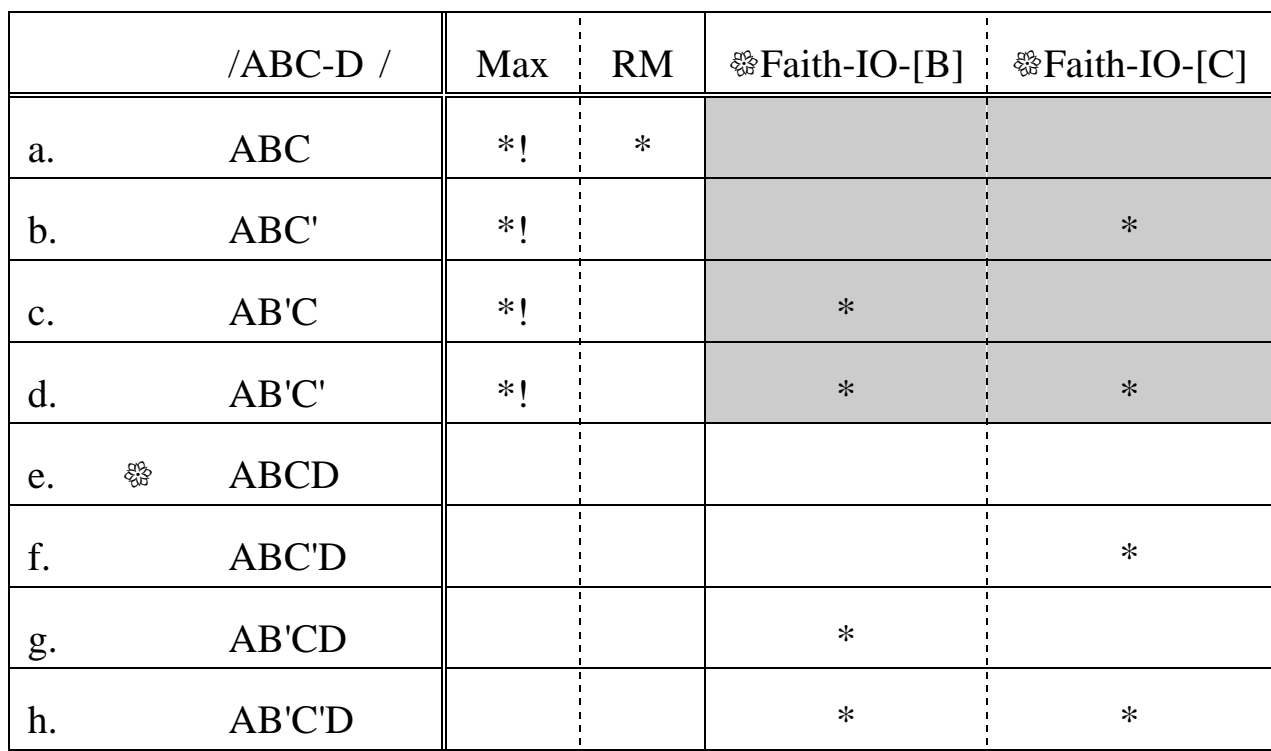

The above argument indicates that multiple sympathy does not succeed in producing triple or more morphemic exponence in the sympathy system here. Integrating the whole discussion developed thus far in this section, we can conclude that Stem $\equiv \mathrm{PrWd}$ is the only eligible selector constraint and that no other (even additional) selector constraint allows for a morpheme to have more than two surface phonological realizations. Therefore, the upper bound of permissible phonological manifestation of a morpheme is strictly restricted under the sympathy system. This 
restrictiveness, if empirically proved to be correct, provides strong evidence in favor of the RMT-based account of DME.

There is another important theoretical prediction arising from the sympathy system: the stem change involved in DME can never be subtractive morphology. Suppose that Max-IO-Seg occupies Faith-IO in (6), yielding the ranking in (61). The important observation is that Max-IO-Seg crucially has dominated status here whereas it is undominated in all cases discussed in earlier sections.

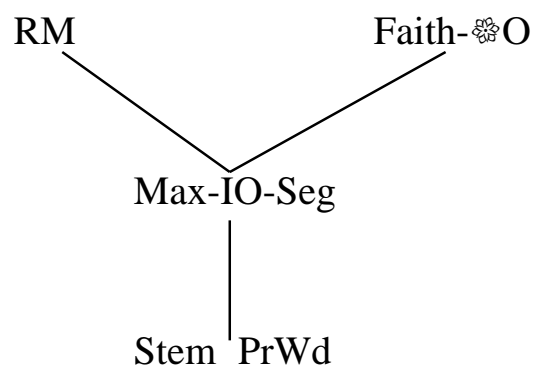

The reason why affixation and subtractive morphology are incompatible becomes clear if we consider a schematic example: /ABC- $\mathrm{D}_{\alpha} /{ }_{\alpha}$. As the dominance effect in Japanese shows, the pertinent Faithbelow RM (see section 5.3.2). (62) illustrates that [ABD] (with both affixation and morphological subtraction) is harmonically bounded by (62c). This suffices as a proof of the non-existence of the cooccurrence of subtractive morphology and affixation. Looking at (62) more closely, however, (62c) violates ${ }^{8} \mathrm{Stem} \equiv \mathrm{PrWd}$, but [ABD] does too. The consequence is that (62a) is nominated for the sympathy candidate. Dep- $\mathrm{O}$ is violated by $[\mathrm{ABD}]$ as well as by $[\mathrm{ABCD}]$, so $[\mathrm{AB}]$ is the winner. The situation is not ameliorated even if Max$[\mathrm{ABC}]$ is selected as the sympathy candidate, and $[\mathrm{ABD}]$ violates Max- $\mathrm{M}$. Thus, [ABD] never wins no matter what kind of Faith-8 $\mathrm{O}$ is employed. 


\begin{tabular}{|c|c|c|c|c|c|c|}
\hline & & $/ \mathrm{ABC}-\mathrm{D}_{\alpha} / \alpha$ & $\mathrm{RM}$ & Dep-8 0 & Max-IO & Stem $\equiv \operatorname{PrWd}$ \\
\hline a. & 8 & $\mathrm{ABC}$ & $* !$ & & $*$ & \\
\hline b. & 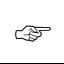 & $\mathrm{AB}$ & & & $* *$ & $*$ \\
\hline c. & & $\mathrm{ABCD}$ & & $* !$ & & $*$ \\
\hline d. & & $\mathrm{ABD}$ & & $* !$ & $*$ & $*$ \\
\hline
\end{tabular}

This is again an important theoretical prediction distinguishing RMT from anti-faithfulness theory. Because nothing prevents the possibility of $\neg$ Max $\gg$ Max in the context of DME, anti-faithfulness theory allows for the cooccurrence of affixation and subtractive morphology as simultaneous phonological instantiations of one and the same morpheme. No such example has been reported, however, to the best of my knowledge.

Summing up this section, I discussed two main theoretical predictions made by the sympathy account of DME. First, a single morpheme can receive maximally two phonological exponents. Given the fact that overt phonological realization sometimes fails as discussed in earlier chapters, the range of phonological exponents for one morpheme is between zero and two. This property is closely associated with a general property of nonconcatenative morphology that maximally only one phonological exponent is possible when a morpheme does not possess any phonological substance. Second, it is predicted by the proposed sympathy system that subtractive morphology and affixation are antagonistic to each other. These two predictions were both couched in terms of harmonic bounding considerations. By contrast, anti-faithfulness theory lacks these predictions, and therefore, it is potentially possible for natural languages to have more than two phonological exponents for a single morpheme as well as to combine affixation and morphological 
subtraction. Exhaustive survey of all human languages is beyond anyone's capacity, and therefore, these two predictions must be tested against more comprehensive data in the future. If they are empirically proved to be correct, the sympathy account for DME embedded in RMT is strongly supported.

\section{$5.8 \quad$ Summary}

In this chapter, I investigated various DME phenomena attested in languages with no genetic relation. I argued that sympathy theory offers a satisfactory analysis. The gist of the proposal is that an affixal element contained in the underlying representation is made opaque such that it behaves as if it were absent for the purpose of satisfying RM. This is formally achieved by assuming Stem $\equiv$ PrWd as the universal selector constraint, which requires perfect correspondence between domains of a stem and a prosodic word. The consequence is that the stem is required to modify its phonological shape. The specific modification is determined by the specific Faith-IO ranked lower than RM. The sympathy candidate exercises its influence over other candidates through Faith-80 Since Max-IO-Seg is undominated in the proposed schema of DME, the affixal element must have a correspondent, resulting in two exponents of a single morpheme.

The sympathy theoretic implementation couched in RMT would be superior to anti-faithfulness theory in terms of formal restrictiveness. I pointed out that there are two theoretical predictions that distinguish the sympathy system and anti-faithfulness theory. The sympathy account for DME predicts first that a single morpheme can possess maximally two phonological exponents and second that affixation and subtractive morphology cannot cooccur as phonological realizations of the same 
morpheme. On the other hand, anti-faithfulness theory is free from these restrictions, and therefore, it predicts first that natural languages do not have any upper bound of the number of phonological exponents of a morpheme and second that affixation is compatible with subtractive morphology. I also argued that more than two phonological exponents of a single morpheme can never be obtained mechanically even if Stem $\equiv$ PrWd is replaced by some other faithfulness constraint or even if more than one selector constraint is posited. These predictions must be tested against more extensive data in the future.

Finally, it is worth pointing out that the rarity of DME in natural languages and the sympathy system could have a correlation. It is empirically supported that inter-candidate correspondence crucial in sympathy theory is not often essential. Sympathetic correspondence is not as extensive as IO-correspondence, for instance. Given the crosslinguistic fact that DME is not observed as often as single morphemic exponence, the rarity of co-candidate correspondence could be fruitfully linked to the infrequency of DME. 


\section{Chapter 6}

\section{Conclusion}

Throughout this dissertation, I addressed various questions surrounding realizational morphology with special attention to nonconcatenative morphology. To close this dissertation, I summarize the main results discussed in the earlier chapters.

To begin with, I argued that two influential morphological models (i.e., Itemand-Arrangement and Item-and-Process) are both unsatisfactory in capturing the whole range of morphological phenomena attested in human languages. The Itemand-Arrangement model cannot accommodate nonconcatenative morphological processes because a word is taken to consist of a sequence of morphemes. On the other hand, the Item-and-Process model is not restrictive in the sense that potentially any type of process could be incorporated in this view, including many unattested ones. In a nutshell, the two morphological models suffer from complementary problems. Given that morphemes manifest their presence through some phonological expression, it is natural that morphological operations take advantage of the same range of processes as those eligible in phonology (cf. Martin 1988), but the immediate question was how to capture this morphology-phonology correspondence. I argued in this light that OT offers an appropriate analytical tool. Phonological alternations are motivated through the existence of markedness constraints ranked over faithfulness constraints, and therefore, possible phonological changes are restricted to those violating the limited set of faithfulness constraints. Because the faithfulness constraints needed in phonology have been fairly established since the

development of correspondence theory (McCarthy and Prince 1995), the extension of OT to realizational morphology is a natural move. 
Given this set-up, I investigated the formal nature of nonconcatenative morphology in chapter 2. The most important observation was that purely morphologically motivated phonological changes exhibit anti-faithfulness effects (cf. Alderete 1999). Since no phonological factor enters the picture as the driving force of such stem modifications, phonological markedness constraints do not play a central role. Under the standard assumption that Con contains only markedness and faithfulness constraints, however, anti-faithfulness effects cannot be captured. This is because anti-faithfulness effects involved in nonconcatenative morphology generate an output which is phonologically more marked than the input, the state of affairs which is never found in markedness-driven phonological alternations. I proposed RM as a universal constraint, whose definition is reproduced in (1). RM requires every morpheme in the underlying representation to receive some phonological exponence on the surface, so it is considered as a morphological faithfulness constraint in the sense that it refers to the morpheme information present in the underlying representation.

(1) Realize Morpheme (RM):

Let $\alpha$ be a morphological form, $\beta$ be a morphosyntactic category, and $\mathrm{F}(\alpha)$ be the phonological form from which $F(\alpha+\beta)$ is derived to express a morphosyntactic category $\beta$. Then $\mathrm{RM}$ is satisfied with respect to $\beta$ iff $\mathrm{F}(\alpha+\beta) \neq \mathrm{F}(\alpha)$ phonologically.

The use of RM per se is not a new proposal, as proposed and employed by many earlier works (Samek-Lodovici 1993; Akinlabi 1996; Gnanadesikan 1997; Rose 1997; Walker 1998, 2000; Piggott 2000; Kurisu 1999, 2000ab, 2001, to appear). I proposed a formalization of the constraint, and developed a significantly expanded argument for the importance of the constraint. Given morphemes as primitive 
morphological units, RM requires every morpheme in the underlying representation to receive some phonological exponence. The specific instantiation of the phonological exponence is not determined by RM. Rather, interactions of constraints determine how a given morpheme should be phonologically manifested in the surface representation. As amply demonstrated, this is especially important when a given morpheme does not contain any phonological substance as part of its information. Under RMT developed here, nonconcatenative morphology is derived by RM » Faith. This general schema directly captures the fact that stem changes invoked in nonconcatenative morphology are morphologically conditioned because they sacrifice phonological faithfulness constraints to satisfy the higher ranked morphological faithfulness constraint. The specific stem modification is determined by the particular phonological faithfulness constraint ranked below RM. This way of understanding realizational morphology enables a formal distinction between purely phonological processes and morphologically governed ones. While the former is motivated by phonological markedness constraints outranking faithfulness ones, the latter is motivated by RM.

I proposed a specific model of how RM violations are computed. First, morphosyntactic categories are derived from bare stems unless there is evidence that a certain morphosyntactic category is derived from another (the input and candidates are directly compared in such cases). Bare stems are forms which crucially lack morphosyntactic information, and their output forms are computed by the grammar. The candidates produced by Gen to evaluate the optimal form of a given base for a certain morphosyntactic category are compared with the output of the bare stem for the purpose of evaluating the satisfaction/violation of RM, as repeated in (2) below. 
This procedure is not only necessary given the richness of the base hypothesis but also desirable in that only morphological factors are taken into account, abstracting away from the influence of purely phonological effects. The pivotal idea is to segregate morphosyntactic functions from stems. Given cases where a certain morphosyntactic category does not have any plausible category as its base, the notion of bare stems is empirically necessary, as discussed in section 2.3.

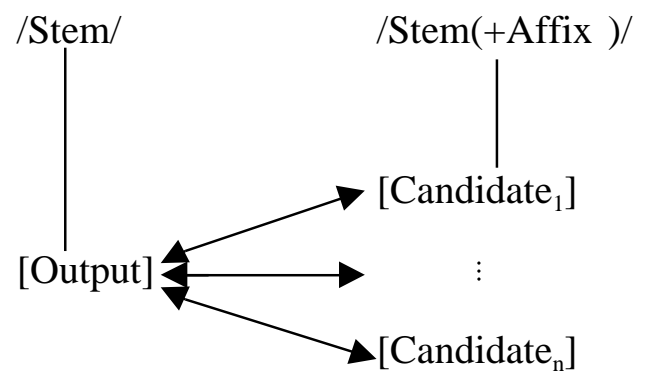

RM requires the two output forms (one of which is typically the output of a bare stem) to be phonologically non-identical, and therefore, it may appear to be the same as anti-faithfulness constraints. They are significantly different in a number of respects. First, anti-faithfulness constraints are descriptively over-powerful in that they call for specific stem modifications, whereas RM does not have such power. Second, anti-faithfulness constraints are operative only when the input is itself an actually occurring output form, but the output of a bare stem does not have to be an actual surface form for RM. This difference is empirically important since there are cases where a certain morphosyntactic category comes from forms which do not stand on their own as independent prosodic words, as in the nominative formation in Lardil. As discussed, the surface-to-surface restriction imposed on anti-faithfulness constraints cannot be dispensed with to avoid undesirable phonological polarity 
effects in the lexical-to-surface dimension. This point thus strongly suggests that RMT is more plausible than anti-faithfulness theory in a large context.

Given RM as the impetus of stem modifications in nonconcatenative morphology, I proposed a general ranking schema to account for them: Faith ${ }_{\alpha} \gg$ RM » Faith $_{\beta}$. The gist of the idea is the relativization of faithfulness constraints with respect to morphosyntactic functions. Since stem modifications do not take place indiscriminately irrespective of morphosyntactic categories, their faithfulness values must be ranked differently with respect to RM. The general ranking schema above explains an asymmetrical behavior of the two morphosyntactic categories $\alpha$ and $\beta$ : Faith is violated in $\beta$ to satisfy RM, but RM must be sacrificed to satisfy Faith in $\alpha$. This means that $\alpha$ does not receive any phonological exponence on the surface. A conceivable alternative is relativizing $R M$, yielding $\mathrm{RM}_{\beta}$ » Faith » $\mathrm{RM}_{\alpha}$. This possibility results in a ranking paradox, however, when some phonological markedness constraint enters the picture to shape the output of the derived category. This indicates that RM is literally a primitive atomic constraint. In the generalized schema, Faith is a variable that is replaced by a range of specific faithfulness constraints. Since reduplication and deletion violate Integrity and Max respectively, one desirable consequence is that we can eliminate abstract but process-specific underlying morphemes such as RED and TRUNC. Given the system that succeeds in obtaining the reduplication and truncation effects, there is no substantive evidence for such morphemes.

RMT has a number of significant theoretical implications. First, it is neither Item-and-Arrangement nor Item-and-Process. It is similar to Item-and-Arrangement in that morphemes are taken to be entities. But the morphological system developed 
here successfully handles morphological operations other than affixation, overcoming the empirical limitation of Item-and-Arrangement. On the other hand, RMT is akin to Item-and-Process too in the sense that linear arrangements of morphs are not what constitutes word formation. But morphemes are taken to be substantial entities, so my view departs from Item-and-Process too. I argued that the extreme pursuit of the Item-and-Process view within the framework of OT attempted by Russell (1995, 1999) is not successful. Second, haplology violates RM if it takes place between adjacent segments without changing phonological features. Because RM is strictly defined in terms of phonological (non-)identity, morphological affiliations of phonological elements have no role to play. Morphological haplology has been considered as a special case where one and the same phonological material manifests the presence of more than one morpheme, but only phonologically tangible manifestation counts as a contribution to the satisfaction of RM. This line of thought would be more promising from the perspective of language processing given the transparent computation of RM.

Building on the argument developed in chapter 2, I examined morphological truncation in chapter 3. Two types of morphological truncation are observed in natural languages: subtractive morphology and templatic truncation. The most important difference is that the deleted portion is constant in subtractive morphology whereas the residue of morphological clipping is prosodically invariable in templatic truncation. Despite this remarkable difference, I argued that both types of truncation are regulated by the same underlying principle. They are required to achieve phonological realization of the relevant morpheme. Given the general schema encapsulated above, $\mathrm{RM} \gg \mathrm{Max}$ is the operative ranking. The formal difference 
between subtractive morphology and templatic truncation is explained by the presence/absence of a set of constraints deriving a templatic effect. Since such constraints are ranked over Max in templatic truncation, the size of the eventual output can be defined prosodically. On the other hand, subtractive morphology is not governed by such prosodic constraints, and therefore, the residue of truncation varies depending on the phonological size of the base.

Another important difference of the two kinds of truncation processes is that subtractive morphology is associated with various grammaticalized word formation (i.e., categorical changes are involved) whereas templatic truncation is employed in non-grammaticalized word formations such as hypocoristics. Put differently, a large portion of the base is preserved in grammaticalized word formations while only a small portion of base information remains in non-grammaticalized truncation. The reason behind this robust difference is a matter left open for future research, but I suggest my speculation here. As discussed in chapter 3, grammaticalized word formation processes are indispensable in the sense that their absence results in serious communication problems while the lack of non-grammaticalized morphology such as hypocoristics does not cause communication troubles. Thus, one possibility to account for the difference would be to claim that preservation of the base information is important in grammaticalized morphology compared with non-grammaticalized one. This idea would be captured by hypothesizing that paradigm uniformity (Kenstowicz 1996; Burzio 1998) plays an active role in regulating the similarity of grammaticalized forms but it is not operative in non-grammaticalized ones. Given that non-grammaticalized morphemes are not a central part of the linguistic system, they are not governed by the principle of paradigm uniformity. 
In chapter 4, I discussed cases where a single morpheme exhibits nonconcatenative allomorphs. The essence of the proposal was that they are indications of languages' effort to optimize phonological structure of the output. Various faithfulness constraints are ranked below RM, and the nonconcatenative allomorph violating the lowest faithfulness constraint is chosen as the default. But other allomorphs are employed when the default option creates a phonologically marked representation. This idea is implemented through interactions of faithfulness and markedness constraints, a central tenet of OT. This way of understanding nonconcatenative allomorphs is parallel to the distribution of affixal allomorphs such as the plural morpheme $-s$ in English in the sense that the phonologically least marked allomorph appears on the surface. This suggests that it is not desirable to comprehend concatenative and nonconcatenative morphology through two distinct mechanisms. Indeed, the integration of them is not only desirable but also necessary. In the discussion of the actual aspect formation in Saanich, I presented a case where they coexist in the realization of a single morpheme. The actual aspect morpheme contains a glottal stop as its phonological substance, but some nonconcatenative stem change is employed even at the expense of a Max violation when glottal stop infixation results in a highly marked phonological representation. Such cases cannot be understood satisfactorily without an integrated system of concatenative and nonconcatenative morphology, so they must be unified under the rubric of realizational morphology.

Nonconcatenative allomorphs also present a serious challenge to antifaithfulness theory. Under this theory, multiple anti-faithfulness constraints need to be ranked over their faithfulness counterparts. Given that anti-faithfulness constraints 
are always in conflict with their faithfulness counterparts, there is no systematic way to prevent the appearance of multiple anti-faithfulness effects for a single morpheme. In other words, anti-faithfulness theory offers no principled way to capture the complementary distribution of various nonconcatenative allomorphs. Furthermore, the actual aspect formation in Saanich counteracts with anti-faithfulness theory since some stem modification is expected to occur in addition to the glottal stop if the actual aspect morpheme activates the strength of anti-faithfulness constraints, contrary to fact. RMT does not lead to the same problem because there is no intrinsically conflictive constraint in the system. This accords with the fundamental idea of OT that which constraints are in conflict with one another differs depending upon the given phonological context.

Finally, in chapter 5, I investigated examples where a single morpheme receives two phonological exponents. The main question was how a stem modification is motivated in a principled manner. I developed the idea of morphological opacity, the effect for the underlying affixal element to be invisible for the purpose of calculating the satisfaction/violation of RM. Morphological opacity is formally captured through sympathy theory (McCarthy 1999), where Stem $\equiv \mathrm{PrWd}$ serves as the selector constraint. It requires the stem domain to be exactly coextensive with a prosodic word domain. This idea is more formally expressed in the form of propositional logical constraint conjunction of Anchor-L(Stem,PrWd), Anchor-R(Stem,PrWd) and Contiguity-Stem. Given this selector constraint, the sympathy candidate is expected to underparse the affixal element contained in the underlying representation. But the candidate undergoes some stem modification under the pressure of RM that is ranked over some Faith-IO. The specific stem 
change depends upon the particular IO-faithfulness constraint. The relevant FaithO $\mathrm{O}$ » Faith-IO ranking requires the ultimate output to mimic the stem change property of the sympathy candidate. Finally, Max-IO-Seg » Stem $\equiv P r W d$ ensures that the affixal segments ultimately appear on the surface. The sympathy system can handle not merely various cases of double morphemic exponence but also subsume the phonological polarity effect in Luo, an important case alleged by Alderete (1999) to constitute strong empirical support for the necessity of anti-faithfulness constraints. Since RMT successfully handles the same data, the plural formation in Luo does not constitute convincing empirical support for anti-faithfulness theory.

Furthermore, I discussed that RMT and anti-faithfulness theory make different empirical predictions in the context of double morphemic exponence. They are concerned with possible and impossible morphology in natural languages. First, I discussed that the sympathy account predicts that a single morpheme receives maximally two phonological exponents. This is because only the affixal element is made invisible, and therefore, one stem modification is sufficient for the satisfaction of RM. No more phonological manifestation is motivated. This property is closely related to the prediction of RMT concerning nonconcatenative morphology in general. When no affix is involved, maximally one stem modification is permitted. Since double morphemic exponence is a mix of concatenative and nonconcatenative morphology, the restriction imposed on nonconcatenative morphology must be obeyed by double morphemic exponence too. I argued that this prediction follows from the consideration of harmonic bounding. Anti-faithfulness theory, on the other hand, predicts that these restrictions do not exist at all. Since potentially an unlimited number of anti-faithfulness constraints can outrank corresponding faithfulness 
constraints, the number of stem changes invoked for morphological reasons is not restricted. But it is unlikely that a single morpheme receives ten phonological exponents, for instance.

Second, the sympathy account predicts that affixation and subtractive morphology never cooccur. Again, this prediction follows from the logic of harmonic bounding. But anti-faithfulness theory allows for the possibility of $\neg$ Max » Max in a context where affixation is involved. This shows that affixation and subtractive morphology are expected to be compatible with each other. Although a more exhaustive survey of morphology in various languages remains to be done, these predictions distinguish the two theories on empirical grounds.

In the investigation of these (especially nonconcatenative) morphological processes, RM plays a central role. The specific phenomena studied in this dissertation (i.e., morphological truncation, nonconcatenative allomorphs, and double morphemic exponence) might appear unrelated to one another superficially, but they are all subsumed under the rubric of realizational morphology. All such morphological phenomena are motivated to obtain some phonological incarnation of morphemes. There are remaining problems, however. I investigated systematic cases in this dissertation, but the most notable question is how suppletive morphology in English is to be accounted for, for example. It is not clear how suppletion as in foot $\approx$ feet should be captured in the overall context of this work. Given the fact that English has various strategies to realize the plural morpheme (i.e., $-s$ suffixation

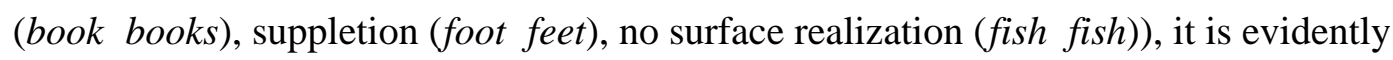
insufficient to allow for faithfulness constraints relativized with respect to morphosyntactic functions. An immediate question is how this kind of morpheme- 
specific behavior should be explained in a principled way. One obvious possibility would be to separate a single morpheme into subclasses and make relativized faithfulness constraints sensitive to those subdivided morphosyntactic categories, as actually done in the analysis of Koasati plurals in section 3.2.1. Under this view, Ident-IO-[back] associated with the plural morpheme relevant to suppletion is ranked below RM. This is along the lines of the proposal made in this dissertation, but it awaits further intensive study to understand whether this is the most plausible approach to such rather irregular morphology. 


\section{References}

Akinlabi, Akinbiyi. (1996). Featural Affixation. Journal of Linguistics 32. 239-289.

Alderete, John. (1999). Morphologically Governed Accent in Optimality Theory. Doctoral dissertation. University of Massachusetts, Amherst.

Alejándro, Rufino. (1963). Handbook of Tagalog Grammar (with Exercises). Manila: University Publishing Company.

Allan, Robin, Philip Holmes and Tom Lundskœr-Nielsen. (1995). Danish: A Comprehensive Grammar. London and New York: Routledge.

Anderson, Stephen. (1975). On the Interaction of Phonological Rules of Various Types. Journal of Linguistics 11. 39-62.

Anderson, Stephen. (1988). Morphological Theory. In Frederick Newmeyer (ed.). Linguistics: The Cambridge Survey 1. 146-191.

Anderson, Stephen. (1992). A-Morphous Morphology. Cambridge: Cambridge University Press.

Anderson, Stephen and Wayles Browne. (1973). On Keeping Exchange Rules in Czech. Papers in Linguistics 6. 445-482.

Anttila, Raimo. (1989). Historical and Comparative Linguistics. $2^{\text {nd }}$ revised edition. Amsterdam: John Benjamins Publishing Company.

Arnason, Kristján. (1980). Quantity in Historical Phonology: Icelandic and Related Cases. Cambridge, Massachusetts: MIT Press.

Aronoff, Mark. (1976). Word Formation in Generative Grammar. Cambridge, Massachusetts: MIT Press.

Bach, Emmon and Robert King. (1970). Umlaut in Modern German. Glossa 4. 3-21.

Bagemihl, Bruce. (1989). The Crossing Constraint and 'Backwards Languages'. Natural Language and Linguistic Theory 7. 481-549.

Bat-El, Outi. (2000). Anti-Faithfulness: An Inherent Morphological Property. Paper presented at the $5^{\text {th }}$ International Conference of Afroasiatic Languages. Paris VII. June 28-30, 2000.

Beckman, Jill. (1995). Shona Height Harmony: Markedness and Positional Identity. In Jill Beckman, Laura Walsh-Dickey, and Suzanne Urbanczyk (eds.). University of Massachusetts Occasional Papers in Linguistics 18: Papers in Optimality Theory. 53-75. 
Beckman, Jill. (1997). Positional Faithfulness, Positional Neutralization, and Shona Vowel Harmony. Phonology. 14. 1-46.

Benua, Laura. (1995). Identity Effects in Morphological Truncation. In Jill Beckman, Laura Walsh-Dickey, and Suzanne Urbanczyk (eds.). University of Massachusetts Occasional Papers in Linguistics 18: Papers in Optimality Theory. 77-136.

Benua, Laura. (1997). Transderivational Identity: Phonological Relations between Words. Doctoral dissertation. University of Massachusetts, Amherst.

Besnier, Niko. (1987). An Autosegmental Approach to Metathesis in Rotuman. Lingua 73. 201-223.

Bessell, Nicola and Ewa Czaykowska-Higgins. (1991). Interior Salish Evidence for Placeless Laryngeals. Proceedings of the North East Linguistic Society 22. 3549.

Biggs, Bruce. (1959). Rotuman Vowels and their History. Te Reo 2. 24-26.

Biggs, Bruce. (1965). Direct and Indirect Inheritance in Rotuman. Lingua 14. 383415.

Bird, Steven. (1990). Constraint-Based Phonology. Doctoral dissertation. University of Edinburgh.

Blake, Ringgold. (1925). A Grammar of the Tagalog Language. American Oriental Series, 1. New Haven, Connecticut: American Oriental Society.

Blevins, Juliette. (1994). The Bimoraic Foot in Rotuman Phonology and Morphology. Oceanic Linguistics 33. 491-516.

Blevins, Juliette. (1997). Rules in Optimality Theory: Two Case Studies. In Iggy Roca (ed.). Derivations and Constraints in Phonology. Oxford: Clarendon Press. 227-260.

Bloomfield, Leonard. (1933). Language. New York: Holt.

Broadwell, George. (1993). Subtractive Morphology in Southern Muskogean. International Journal of American Linguistics 59. 416-429.

Bromberger, Sylvain and Morris Halle. (1988). Conceptual Issues in Morphology. Ms. MIT.

Buckley, Eugene. (1998). Integrity and Correspondence in Manam Double Reduplication. Proceedings of the North East Linguistic Society 28. 59-67.

Burzio, Luigi. (1998). Multiple Correspondence. Lingua 104. 79-109. 
Cairns, Charles. (1976). Universal Properties of Umlaut and Vowel Coalescence Rules: Implications for Rotuman Phonology. In Alphonse Juilland (ed.). Linguistic Studies Offered to Joseph Greenberg. vol. 2 (Phonology). Saratoga, California: Anma Libri. 271-283.

Carrier, Jill. (1979). The Interaction of Morphological and Phonological Rules in Tagalog: A Study in the Relationship between Rule Components in Grammar. Doctoral dissertation. MIT.

Carrier, Jill. (1984). Some Problems with Prosodic Accounts of Reduplication. In Mark Aronoff and Richard Oehrle (eds.). Language Sound Structure. Cambridge, Massachusetts: MIT Press. 260-286.

Chomsky, Noam. (1951). Morphophonemics of Modern Hebrew. Master thesis. University of Pensylvania.

Chomsky, Noam and Morris Halle. (1968). The Sound Pattern of English. New York: Harper and Row.

Churchward, Maxwell. (1940). Rotuman Grammar and Dictionary. Sydney: Australasia Medical Publishing Co..

Clements, (1990). The Role of the Sonority Cycle in Core Syllabification. In John Kingston and Mary Beckman (eds.). Papers in Laboratory Phonology 1. Cambridge: Cambridge University Press. 283-333.

Crowhurst, Megan. (1998). Um Infixation and Prefixation in Toba Batak. Language 74. 590-604.

Crowhurst, Megan and Mark Hewitt. (1997). Boolean Operations and Constraint Interactions in Optimality Theory. Ms. University of North Carolina, Chapel Hill and Brandeis University. [ROA-299-1197].

Davis, Philip. (1995). The Way of Language: Dimensions of Voice. In Philip Davis (ed.). Alternative Linguistics: Descriptive and Theoretical Models. Amsterdam: Benjamins.

Davis, Philip, John Baker, Walter Spitz and Mihyun Baenk. (1996). Yogad: A Functional Explanation. (LINCOM Studies in Austronesian Linguistics). Munich: LINCOM Europa.

Davis, Stuart and Isao Ueda. (2001). Morphological Mora Augmentation. Paper presented at the fifth Holland Institute of Linguistics Phonology Conference. University of Potsdam. January 11.

Dressler, Wolfgang. (1987). Subtraction in a Polycentristic Theory of Natural Morphology. In Edmund Gussmon (ed.). Rules and the Lexicon: The Study in Word Formation. Lublin: Katholickiego Uniwersytetu Lubelskiego. 67-77. 
Elmedlaoui, Mohamed. (1992). Aspects des Représentations Phonologiques dans Certaines Langues Chamito-Semitiques. Doctoral dissertation. Université Mohamed V, Rabat.

Fitzgerald, Colleen. (1997). O'odham Rhythms. Doctoral dissertation. University of Arizona.

Flemming, Edward. (1995). Auditory Representations in Phonology. Doctoral dissertation. University of California, Los Angeles.

French, Koleen. (1988). Insights into Tagalog Reduplication, Infixation, and Stress from Nonlinear Phonology. Dallas, Texas: The Summer Institute of Linguistics.

Fukazawa, Haruka, Mafuyu Kitahara and Mitsuhiko Ota. (1998). Lexical Stratification and Ranking Invariance in Constraint-Based Grammars. Proceedings of the Chicago Linguistic Society 34: The Panels. 47-62.

Galloway, Brent (1980). The Structure of Upriver Halq'eméylem: A Grammatical Sketch and Classified Word List for Upriver Halq'eméylem. Sardis, British Columbia: Coqualeetza Education Training Centre.

Galloway, Brent. (1993). A Grammar of Upriver Halkomelem. University Publications in Linguistics 96. Berkeley: University of California Press.

Gnanadesikan, Amalia. (1997). Phonology with Ternary Scales. Doctoral dissertation. University of Massachusetts, Amherst.

Goldsmith, John. (1976). Autosegmental Phonology. Doctoral dissertation. MIT. [Published from Garland Press, 1979].

Golston, Chris. (1996). Direct Optimality Theory: Representation as Pure Markedness. Language 72. 713-748.

Golston, Chris and Richard Wiese. (1995). Zero Morphology and Constraint Interaction: Subtraction and Epenthesis in German Dialects. In Geert Booij and Jaap van Marle (eds.). Yearbook of Morphology 1995. Dordrecht: Kluwer Acadmic Publishers. 143-159.

Gregersen, Edgar. (1972). Consonant Polarity in Nilotic. Proceedings of the Third Annual Conference on African Linguistics. 105-109.

Hale, Kenneth. (1965). Some Preliminary Observations on Papago Morphophonemics. International Journal of American Linguistics 31. 295305 .

Hale, Kenneth. (1973). Deep-Surface Canonical Disparities in Relation to Analysis and Change: An Australian Example. Current Trends in Linguistics 11. 401458. 
Hale, Kenneth and Abanel Blanco. (1989). Diccionario Elemental del Ulwa (Sumu Meridional). Center for Cognitive Science, MIT.

Hale, Mark and Madelyn Kissock. (1998). The Phonology-Syntax Interface in Rotuman. Proceedings of the Third and Fourth Meetings of the Austronesian Formal Linguistics Association. UCLA Occasional Papers in Linguistics \#21. UCLA Department of Linguistics, Los Angeles. 115-128.

Hammond, Michael. (1995). There is no Lexicon!. Ms. University of Arizona.

Harris, Zelig. (1941). Linguistic Structure of Hebrew. Journal of the American Oriental Society 62. 143-167.

Harris, Zelig. (1945). Discontinuous Morphemes. Language 21. 121-127.

Haudricourt, Georges. (1958a). L'accent D'agencement en Mélanésien de Rotuma. Bulletin de la Société de Linguistique de Paris 53. 16-17.

Haudricourt, Georges. (1958b). La Phonologie des Voyelles en Rotumien (Océanie). Bulletin de la Société de Linguistique de Paris 53. 268-272.

Hayes, Bruce. (1989). Compensatory Lengthening in Moraic Theory. Linguistic Inquiry 20. 253-306.

Hayes, Bruce. (1997). Anticorrespondence in Yidi^^. Ms. University of California, Los Angeles.

Hayes, Bruce. (1999). Phonological Restructuring in Yidi^ and its Theoretical Consequences. In Ben Hennans and Marc Oostendorp (eds.). The Derivational Residue in Phonological Optimality Theory. Amsterdam: John Benjamins. 175-205.

Hetzron, Robert. (1971). Internal Labialization in the $t t$-Group Outer South-Ethiopic. Journal of the American Oriental Society 91. 192-207.

Hetzron, Robert. (1977). The Gunnän-Gurage Languages. Naples: Instituto Orientale di Napoli.

Hewitt, Mark. (1994). Deconstructing Foot Binarity in Koniag Alutiiq. Ms. University of British Columbia. [ROA-12-0000].

Hewitt, Mark and Megan Crowhurst. (1996). Conjunctive Constraints and Templates. Proceedings of the North East Linguistic Society 26. 101-116.

Hill, Jane and Ofelia Zepeda. (1992). Derived Words in Tohono O'odham. International Journal of American Linguistics 58. 355-404.

Hockett, Charles. (1947). Problems of Morphemic Analysis. Language 23. 321-343. 
Hockett, Charles. (1950). Peiping Morphemics. Language 26. 63-85.

Hockett, Charles. (1954). Two Models of Grammatical Description. Word 10. 210234.

Hoeksema, Jack and Richard Janda. (1988). Implications of Process-Morphology for Categorial Grammar. In Richard Oehrle, Emmon Bach, and Deirdre Wheeler (eds.). Categorial Grammars and Natural Language Structures. Dordrecht, Holland: Reidel Publishing Company. 199-247.

Horwood, Graham. (1999). Anti-faithfulness and Subtractive Morphology. Ms. Rutgers University.

Hukari, Tomas. (1978). Halkomelem Nonsegmental Morphology. Proceedings of the $13^{\text {th }}$ International Conference on Salishan Languages. 157-209.

van der Hulst, Harry. (1983). Rotuman Umlaut. Ms. University of Leiden.

Hyman, Larry. (1985). A Theory of Phonological Weight. Dordrecht: Foris.

Inkelas, Sharon. (1999). Exceptional Stress Attracting Suffixes in Turkish: Representations versus the Grammar. In René Kager, Harry van der Hulst, and Wim Zonneveld (eds.). The Prosody-Morphology Interface. Cambridge: Cambridge University Press. 134-187.

Inkelas, Sharon, Orhan Orgun and Cheryl Zoll. (1997). The Implications of Lexical Exceptions for the Nature of Grammar. In Iggy Roca (ed.). Derivations and Constraints in Phonology. Oxford: Clarendon Press. 393-418.

Itô, Junko. (1986). Syllable Theory in Prosodic Phonology. Doctoral dissertation. University of Massachusetts, Amherst. [Published from Garland Press, 1988].

Itô, Junko. (1989). A Prosodic Theory of Epenthesis. Natural Language and Linguistic Theory 7. 217-259.

Itô, Junko. (1990). Prosodic Minimality in Japanese. Proceedings of the Chicago Linguistic Society 26-II; Papers from the Parasession on the Syllable in Phonetics and Phonology. 213-239.

Itô, Junko, Yoshihisa Kitagawa and Armin Mester. (1996). Prosodic Faithfulness and Correspondence: Evidence from a Japanese Argot. Journal of East Asian Linguistics 5. 217-294.

Itô, Junko and Armin Mester. (1992). Weak Layering and Word Binarity. Linguistics Research Center. LRC-92-09. University of California, Santa Cruz.

Itô, Junko and Armin Mester. (1995a). Japanese Phonology. In John Goldsmith (ed.). The Handbook of Phonological Theory. Cambridge: Blackwell Publishers. 817-838. 
Itô, Junko and Armin Mester. (1995b). The Core-Periphery Structure of the Lexicon and Constraints on Reranking. In Jill Beckman, Laura Walsh-Dickey, and Suzanne Urbanczyk (eds.). University of Massachusetts Occasional Papers in Linguistics 18: Papers in Optimality Theory. 181-209.

Itô, Junko and Armin Mester. (1998). Sympathy Theory and German Truncations. In the Phonological Society of Japan (ed.). Oninkenkyuu [Phonological Studies] 1. Tokyo: Kaitakusha. 51-66.

Itô, Junko and Armin Mester. (1999a). The Best-of-the-Best in Danish. Lecture handout delivered at the phonology seminar in Fall, 1999. University of California, Santa Cruz.

Itô, Junko and Armin Mester. (1999b). The Phonological Lexicon. In Natsuko Tsujimura (ed.). The Handbook of Japanese Linguistics. Cambridge, Massachusetts: Blackwell Publishers. 62-100.

Itô, Junko, Armin Mester and Jaye Padgett. (1995). Licensing and Underspecification in Optimality Theory. Linguistic Inquiry 26. 571-613.

Janda, Richard. (1982a). Of Formal Identity and Rule-(Un)Collapsibility: On Lost and Found Generalizations in Morphology. Proceedings of the West Coast Conference on Formal Linguistics 1. 179-197.

Janda, Richard. (1982b). On Limiting the Form of Morphological Rules: German Umlaut, Diacritic Features, and the "Cluster-Constraint". Proceedings of the North East Linguistic Society 12. 140-152.

Janda, Richard. (1984). Why Morphological Metathesis Rules are Rare: On the Possibility of Historical Explanation in Linguistics. Proceedings of the Berkeley Linguistic Society 10. 87-103.

Johnson, Douglous. (1975). Phonological Channels in Chaha. Afroasiatic Linguistics 2. 1-13.

Jones, Glyn and Kirsten Gade. (1981). Danish, A Grammar. Copenhagen: Nordisk Forlag A.S.

Katayama, Motoko. (1998). Optimality Theory and Japanese Loanword Phonology. Doctoral dissertation. University of California, Santa Cruz.

Kawahara, Shigeto. (2001). Morphological Blocking of Fusion in German Verbal Paradigm. Ms. University of California, Santa Cruz.

Kenstowicz, Michael. (1996). Base-Identity and Uniform Exponence: Alternative to Cyclicity. Current Trends in Phonology: Models and Methods. In Jacque Durand and Bernard Laks (eds.). Salford, Manchester: European Studies Research Institute, University of Salford. 363-393. 
Kimball, Geoffrey. (1983). Verb Pluralization in Koasati. Proceedings of the 1982 Mid-America Linguistics Conference Papers. 401-411.

Kimball, Geoffrey. (1991). Koasati Grammar. Lincoln: University of Nebraska Press.

Kiparsky, Paul. (1971). Historical Linguistics. In William Dingwall (ed.). A Survey of Linguistic Science. College Park, Maryland: University of Maryland Linguistics Program. 576-642.

Kiparsky, Paul. (1973). Abstractness, Opacity, and Global Rules. In Osamu Fujimura (ed.). Three Dimensions of Linguistic Theory. Tokyo: TEC Company. 57-86.

Kiparsky, Paul. (1984). On the Lexical Phonology of Icelandic. In Claes-Christian Elert, Iréne Johansson, and Eva Strangert (eds.). Nordic Prosody III. University of Umeå. 135-164.

Klosa, Mannheim, Werner Scholze-Stubenrecht, and Matthias Wermke (eds.). (1998). Duden. Die Grammatik: Unentbehrlich für Richtiges Deutsch. 6., Neu Bearbeitete Auflage Herausgegeben von der Dudenredaktion Bearbeitet von Peter Eisenberg, Hermann Gelhaus, Helmut Henne, Horst Sitta und Hans Wellmann. Duden Band 4. Mannheim, Leipzig, Wien, and Zürich: Dudenverlag.

Kurisu, Kazutaka. (1999). Morpheme Realizations and Phonological Exponence. Proceedings of the Second GLOW Conference in Asia. 239-256.

Kurisu, Kazutaka. (2000a). Double Morphemic Exponence as Morphological Opacity. Proceedings of the Chicago Linguistic Society 36. Volume 2: The Panels. 163-177.

Kurisu, Kazutaka. (2000b). Multiple Nonconcatenative Allomorphs. In the Phonological Society of Japan (ed.). Oninkenkyuu [Phonological Studies] 3. Tokyo: Kaitakusha. 53-60.

Kurisu, Kazutaka. (2000c). Richness of the Base and Root Fusion in Sino-Japanese. Journal of East Asian Linguistics 9. 147-185.

Kurisu, Kazutaka. (2001). Morphological Opacity and Chaha Impersonals. In the Phonological Society of Japan (ed.). Oninkenkyuu [Phonological Studies] 4. Tokyo: Kaitakusha. 57-64.

Kurisu, Kazutaka. (to appear). Affixation-Reduplication Interactions and Morphological Opacity in Tagalog. Proceedings of the Western Conference on Linguistics 2000.

de Lacy, Paul. (1999). Morphological Haplology and Correspondence. University of Massachusetts Occasional Papers 24: Papers from the $25^{\text {th }}$ Anniversary. 5189. 
Leslau, Wolf. (1950). Ethiopic Documents: Gurage. New York: Viking Fund Publications in Anthropology.

Leslau, Wolf. (1967). The Impersonal in Chaha. To Honor Roman Jakobson: Essays on the Occasion of His Seventieth Birthday. Vol. II. The Hague: Mouton. 1150-1160.

Lieber, Rochelle. (1981). On the Organization of the Lexicon. Doctoral dissertation. University of New Hampshire.

Lieber, Rochelle. (1988). Configurational and Nonconfigurational Morphology. In Martin Everaert, Arnold Evers, Riny Huybregts, and Mieke Trommelen (eds.). Morphology and Modularity. Dordrecht: Foris Publications. 187-215.

Lieber, Rochelle. (1992). Deconstructing Morphology: Word Formation in Syntactic Theory. Chicago and London: The University of Chicago Press.

Lodge, Norwich. (1989). A Non-Segmental Account of German Umlaut: Diachronic and Synchronic Perspectives. Linguistische Berichte 124. 470-491.

Lombardi, Linda. (1998). Evidence for MaxFeature Constraints from Japanese. Ms. University of Maryland. [ROA-247-0298].

Lombardi, Linda and John McCarthy. (1991). Prosodic Circumscription in Choctaw Morphology. Phonology 8. 37-71.

Maddieson, Ian. (1984). Patterns of Sounds. Cambridge: Cambridge University Press.

Malone, Joseph. (1993). Tiberian Hebrew Phonology. Winona Lake, Indiana: Eisenbrauns.

Marantz, Alec. (1982). Re Reduplication. Linguistic Inquiry 13. 435-482.

Martin, Jack. (1988). Subtractive Morphology as Dissociation. Proceedings of the West Coast Conference on Formal Linguistics 7. 229-240.

McCarthy, John. (1979). Formal Problems in Semitic Phonology and Morphology. Doctoral dissertation. MIT. [Published from Garland Press, 1982].

McCarthy, John. (1981). A Prosodic Theory of Nonconcatenative Morphology. Linguistic Inquiry 12. 373-418.

McCarthy, John. (1983). Consonantal Morphology in the Chaha Verb. Proceedings of the West Coast Conference on Formal Linguistics 2. 176-188.

McCarthy, John. (1986a). Lexical Phonology and Nonconcatenative Morphology in the History of Chaha. Revue Quebecoise de Linguistique 16. 209-228. 
McCarthy, John. (1986b). OCP Effects: Gemination and Antigemination. Linguistic Inquiry 17. 207-263.

McCarthy, John. (1989). Linear Order in Phonological Representation. Linguistic Inquiry 20. 71-99.

McCarthy, John. (1993). A Case of Surface Constraint Violation. Canadian Journal of Linguistics 38. 169-195.

McCarthy, John. (1999). Sympathy and Phonological Opacity. Phonology 16. 331399.

McCarthy, John. (2000a). Faithfulness and Prosodic Circumscription. In Joost Dekkers, Frank van der Leeuw, and Jeroen van de Weijer (eds.). Optimality Theory: Syntax, Phonology, and Acquisition. Oxford: Oxford University Press. 151-189.

McCarthy, John. (2000b). Harmonic Serialism and Parallelism. Proceedings of the North East Linguistic Society 30. 501-524.

McCarthy, John. (2000c). The Prosody of Phase in Rotuman. Natural Language and Linguistic Theory 18. 147-197.

McCarthy, John and Alan Prince. (1986). Prosodic Morphology. Ms. University of Massachusetts, Amherst and Rutgers University. [to appear from MIT Press].

McCarthy, John and Alan Prince. (1990). Word and Foot in Prosodic Morphology: The Arabic Broken Plural. Natural Language and Linguistic Theory 8. 209282.

McCarthy, John and Alan Prince. (1993a). Generalized Alignment. In Geert Booij and Jaap van Marle (eds.). Yearbook of Morphology 1993. Dordrecht: Kluwer Academic Publishers. 79-153.

McCarthy, John and Alan Prince. (1993b). Prosodic Morphology I: Constraint Interaction and Satisfaction. Ms. University of Massachusetts, Amherst and Rutgers University, New Brunswick.

McCarthy, John and Alan Prince. (1994). The Emergence of the Unmarked: Optimality in Prosodic Morphology. Proceedings of the North East Linguistic Society 24. 333-379.

McCarthy, John and Alan Prince. (1995). Faithfulness and Reduplicative Identity. In Jill Beckman, Laura Walsh-Dickey, and Suzanne Urbanczyk (eds.). University of Massachusetts Occasional Papers in Linguistics 18: Papers in Optimality Theory. 249-384.

McCarthy, John and Alan Prince. (1999). Faithfulness and Identity in Prosodic Morphology. In René Kager, Harry van der Hulst, and Wim Zonneweld (eds.). 
The Prosody-Morphology Interface. Cambridge: Cambridge University Press. 218-309.

McCawley, James. (1968). The Phonological Component of a Grammar of Japanese. The Hague: Mouton.

Mester, Armin. (1986). Studies in Tier Structure. Doctoral dissertation. University of Massachusetts, Amherst. [Published from Garland Press, 1988].

Mester, Armin. (1990). Patterns of Truncation. Linguistic Inquiry 21. 478-485.

Milner, George. (1971). Fijian and Rotuman. In Thomas Sebeok (ed.). Current Trends in Linguistics. Vol. 8: Linguistics in Oceania. Mouton: The Hague. 397-425.

Montler, Timothy. (1986). Morphology and Phonology of Saanich. Missoula, Montana: University of Montana Publishers.

Montler, Timothy. (1989). Infixation, Reduplication, and Metathesis in the Saanich Actual Aspect. Southwest Journal of Linguistics 9. 92-107.

Moreton, Elliot. (1999). Non-computable functions in Optimality Theory. Ms. University of Massachusetts, Amherst. [ROA-364-1199].

Nababan, Partabas. (1981). A Grammar of Toba-Batak. Pacific Linguistics Series. D37. Canberra, Australia: Department of Linguistics, Research School of Pacific Studies, Australian National University.

Nida, Eugene. (1948). The Identification of Morphemes. Language 24. 414-441.

Odden, David. (1988). Anti Antigemination and the OCP. Linguistic Inquiry 19. 451475.

Okoth-Okombo, Duncan. (1982). Dholuo Morphophonemics in a Generative Framework. Berlin: Dietrich Reimer Verlag.

Oreshnik, Janez. (1972). On the Epenthesis Rule in Modern Icelandic. Arkiv för Nordisk Filologi 87. 1-32.

Oreshnik, Janez. (1978a). The Modern Icelandic Epenthesis Rule Revisited. Arkiv för Nordisk Filologi 93. 166-173.

Oreshnik, Janez. (1978b). The Age and Importance of the Modern Icelandic Type klifr. In John Weinstock (ed.). The Nordic Language and Modern Linguistics 3. 468-471.

Padgett, Jaye. (2001). Contrast Dispersion and Russian Palatalization. In Elizabeth Hume and Keith Johnson (eds.). The Role of Speech Perception Phenomena in Phonology. San Diego: Academic Press. 187-218. 
Pater, Joe. (1995). On the Nonuniformity of Weight-to-Stress and Stress Preservation Effects in English. Ms. McGill University.

Pater, Joe. (1999). Austronesian Nasal Substitution and Other NC Effects. In René Kager, Harry van der Hulst, and Wim Zonneweld (eds.). The ProsodyMorphology Interface. Cambridge: Cambridge University Press. 310-343.

Percival, Keith. (1981). A Grammar of the Urbanized Toba Batak of Medan. Pacific Linguistics B-76. Canberra, Australia: Department of Linguistics, Research School of Pacific Studies, Australian National University.

Piggott, Glyne. (2000). Against Featural Alignment. Journal of Linguistics 36. 85129.

Plag, Ingo. (1998). Morphological Haplology in a Constraint-Based MorphoPhonology. In Wolfgang Kehrein and Richard Wiese (eds.). Phonology and Morphology of the Germanic Langauges. Tübingen: Niemeyer. 199-215.

Polotsky, Hans. (1938). Etudes de Grammarire Gouragué. Bulletin de la Société de Linguistique de Paris 34. 137-175.

Poser, William. (1984a). Hypocoristic Formation in Japanese. Proceedings of the West Coast Conference on Formal Linguistics 3. 218-229.

Poser, William. (1984b). The Phonetics and Phonology of Tone and Intonation in Japanese. Doctoral dissertation. MIT.

Poser, William. (1990). Evidence for Foot Structure in Japanese. Language 66. 78105.

Pranka, Paula. (1983). Syntax and Word Formation. Doctoral dissertation. MIT.

Prince, Alan. (1975). The Phonology and Morphology of Tiberian Hebrew. Doctoral dissertation. MIT.

Prince, Alan. (1996). Aspects of Mapping under OT. Handout from the talk given at the University of California, Santa Cruz. October 11, 1996.

Prince, Alan. (1997). Endogenous Constraints on Optimality Theory. Paper presented at Hopkins Optimality Theory Workshop. University of Maryland Mayfest. May 8-12, 1997.

Prince, Alan and Paul Smolensky. (1993). Optimality Theory: Constraint Interaction in Generative Grammar. Ms. Rutgers University and University of Colorado, Boulder.

Pullum, Geoffrey and Arnold Zwicky. (1991). A Misconceived Approach to Morphology. Proceedings of the West Coast Conference on Formal Linguistics 10. 387-398. 
Ramos, Teresita. (1974). The Case System of Tagalog Verbs. Pacific Linguistics Series, B-27. Canberra, Australia: Department of Linguistics, Research School of Pacific Studies, Australian National University.

Robinson, Orrin. (1975). Abstract Phonology and the History of Umlaut. Lingua 37. $1-29$.

Rosenthall, Samuel. (1994). Vowel/Glide Alternation in a Theory of Constraint Interaction. Doctoral dissertation. University of Massachusetts, Amherst.

Rose, Sharon. (1994). The Historical Development of Secondary Articulation in Gurage. Proceedings of the Berkeley Linguistic Society: Special Session on Historical Issues in African Linguistics 20. 112-124.

Rose, Sharon. (1997). Theoretical Issues in Comparative Ethio-Semitic Phonology and Morphology. Doctoral dissertation. McGill University, Montreal.

Rubach, Jerzy. (1990). Final Devoicing and Cyclic Syllabification in German. Linguistic Inquiry 21. 79-94.

Russell, Kevin. (1993). A Constraint-Based Approach to Phonology and Morphology. Doctoral dissertation. University of Southern California.

Russell, Kevin. (1995). Morphemes and Candidates in Optimality Theory. Ms. University of Manitoba. [ROA-44-0195].

Russell, Kevin. (1999). MOT: Sketch of an OT Approach to Morphology. Ms. University of Manitoba. [ROA-352-1099].

Saito, Mamoru. (1981). A Preliminary Account of the Rotuman Vowel System. Ms. MIT.

Samek-Lodovici, Vieri. (1993). Morphological Gemination. Paper presented at Rutgers Optimality Workshop I. Rutgers University. October, 1993.

Sanders, Gerald. (1988). Zero Derivation and the Overt Analogue Criterion. In Michael Hammond and Michael Noonan (eds.). Theoretical Morphology: Approaches in Modern Linguistics. 155-175.

Sanders, Nathan. (1999). Same-Edge Alignment with Opposite-Edge Effects. Paper presented at the $18^{\text {th }}$ West Coast Conference on Formal Linguistics. University of Arizona. April 10, 1999.

Sanders, Nathan. (2000). Intra-Representational Correspondence and the Realization of Empty Morphemes. Ms. University of California, Santa Cruz.

Schachter, Paul and Fe T. Otanes. (1972). Tagalog Reference Grammar. Berkeley: University of California Press. 
Scobbie, James. (1991). Attribute Value Phonology. Doctoral dissertation. University of Edinburgh.

Smolensky, Paul. (1993). Harmony, Markedness, and Phonological Activity. Paper presented at Rutgers Optimality Workshop I. Rutgers University. October, 1993.

Smolensky, Paul. (1995). On the Internal Structure of the Constraint Component Con of UG. Paper presented at the University of California, Los Angeles. April 7, 1995. [ROA-86-0000]

Smolensky, Paul. (1996). The Initial State and 'Richness of the Base' in Optimality Theory. Ms. John Hopkins University. [ROA-154-1196].

Smolensky, Paul. (1997). Constraint Interaction in Generative Grammar II: Local Conjunction. Paper presented at Hopkins Optimality Theory Workshop. University of Maryland Mayfest. May 8-12, 1997.

Soberano, Rosa. (1980). The Dialects of Marinduque Tagalog. Pacific Linguistics Series B-69. Department of Linguistics, Research School of Pacific Studies, The Australian National University.

Spaelti, Philip. (1997). Dimensions of Variation in Multi-Pattern Reduplication. Doctoral dissertation. University of California, Santa Cruz. [ROA-311-0499].

Spencer, Andrew. (1991). Morphological Theory. Cambridge: Basil Blackwell Ltd..

Stafford, Roy. (1967). An Elementary Luo Grammar. London: Oxford University Press.

Steriade, Donca. (1982). Greek Prosodies and the Nature of Syllabification. Doctoral dissertation. MIT. [Published from Garland Press, 1990].

Stonham, John. (1994). Combinatorial Morphology. Amsterdam and Philadelphia: John Benjamins Publishing Company.

Strauss, Steven. (1976). The Nature of Umlaut in Modern Standard German. CUNYforum 1. 121-144.

Struijke, Caro. (1998). Reduplicant and Output TETU in Kwakwala. University of Maryland Working Papers: Papers in Phonology 7. 150-178.

Suzuki, Hisami. (1995). Minimal Words in Japanese. Proceedings of the Chicago Linguistic Society. 31: The Main Session. 448-463.

Tateishi, Koichi. (1989). Theoretical Implications of the Japanese Musician's Language. Proceedings of the West Coast Conference on Formal Linguistics 8. 384-398. 
Thompson, Lawrence and Terry Thompson. (1971). Clallam: A Preview. In Jesse Sawyer (ed.). Studies in American Languages. Berkeley, Los Angeles, and London: University of California Press. 251-294.

Topping, Donald. (1973). Chamorro Reference Grammar. Honolulu, Hawaii: The University of Hawaii Press.

Urbanczyk, Suzanne. (1996). Morphological Templates in Reduplication. Proceedings of the North East Linguistic Society 26. 425-440.

Urbanczyk, Suzanne. (1998). A-Templatic Reduplication in Halq'eméylem. Proceedings of the West Coast Conference on Formal Linguistics 17. 655669.

Ussishkin, Adam. (2000). The Emergence of Fixed Prosody. Doctoral dissertation. University of California, Santa Cruz.

Walker, Rachel. (1998). Nasalization, Neutralization, and Opacity Effects. Doctoral dissertation. University of California, Santa Cruz. [Published from Garland Press, 2000].

Walker, Rachel. (2000). Nasal Reduplication in Mbe Affixation. Phonology 17. 65115.

Weeda, Donald. (1992). Word Truncation in Prosodic Morphology. Doctoral dissertation. University of Texas, Austin.

Wiese, Richard. (1996a). Phonological versus Morphological Rules: On German Umlaut and Ablaut. Journal of Linguistics 32. 113-135.

Wiese, Richard. (1996b). The Phonology of German. Oxford: Clarendon Press.

Wilkinson, Karina. (1988). Prosodic Structure and Lardil Phonology. Linguistic Inquiry 19. 325-334.

Zepeda, Ofelia. (1983). A Papago Grammar. Tucson, Arizona: University of Arizona Press.

Zwicky, Arnold. (1967). Umlaut and Noun Plurals in German. Studia Grammatica 6. $35-45$. 